CONCENTRATION AND TRANSPORT DATA FOR

DISSOLVED INORGANIC CONSTITUENTS IN

WATER COLLECTED DURING SEVEN CRUISES ON

THE MISSISSIPPI RIVER AND SOME OF ITS

TRIBUTARIES, JULY 1987-JUNE 1990

by T. I. Brinton, J. R. Garbarino, D. B. Peart, H. E. Taylor, and

R. C. Antweiler

U.S. GEOLOGICAL SURVEY

Open-File Report 94-524 


\section{U.S. DEPARTMENT OF THE INTERIOR \\ BRUCE BABBITT, Secretary}

U.S. GEOLOGICAL SURVEY

Gordon P. Eaton, Director

The use of trade, product, industry, or firm names is for descriptive purposes only and does not imply endorsement by the U.S. Government.

For additional information write to:

Copies of this report can be purchased from:

Chief, Branch of Regional Research

U.S. Geological Survey

U.S. Geological Survey

Box 25046, MS 418

Earth Science Information Center

Denver Federal Center

Open-File Reports Section

Denver, CO 80225

Box 25286, MS 517

Denver Federal Center

Denver, CO 80225 


\section{CONTENTS}

Abstract
Introduction
Sample collection
Sample processing
Quality control
Analytical methods
Analyte concentration and transport data
References

\section{FIGURES}

1. Map showing the Mississippi River and some of its tributaries.........................................................................

2. Schematic diagram of filter apparatus used for filtration of trace-element samples........................................9

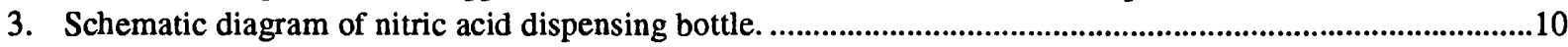

\section{TABLES}

1. Sampling sites on the Mississippi River and some of its tributaries.

2-9. Trace-element concentrations for field blanks taken during the:

2. July-August 1987 cruise on August 9, 1987

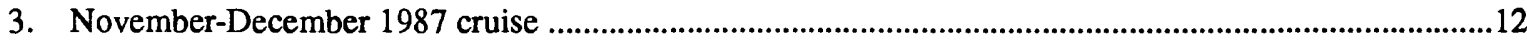

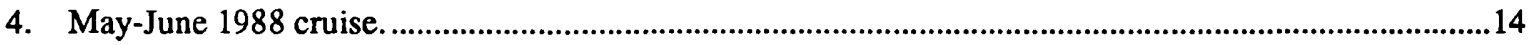

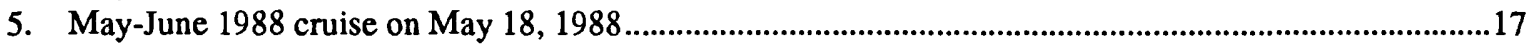

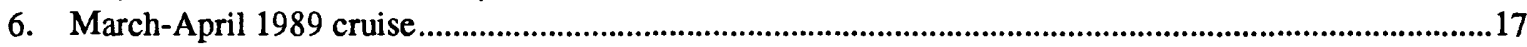

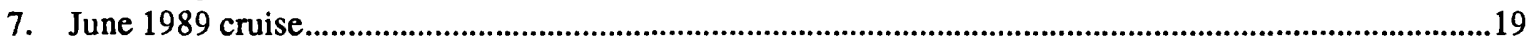

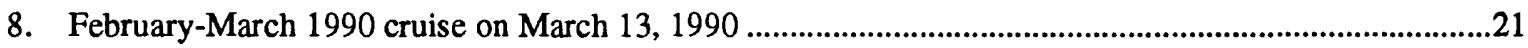

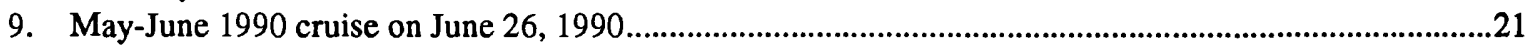

10. Major-element concentrations for field blanks collected during the July-August 1987,

November-December 1987, May-June 1988, March-April 1989, June 1989,

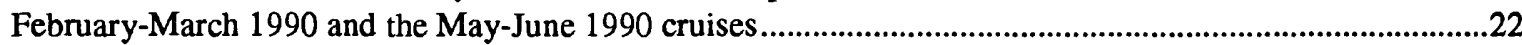

11-17. Quality-control data for analyses of samples from the:

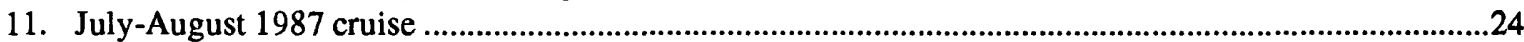

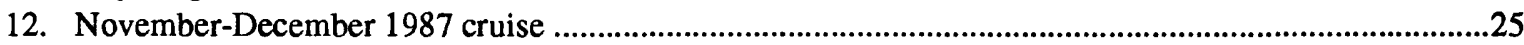

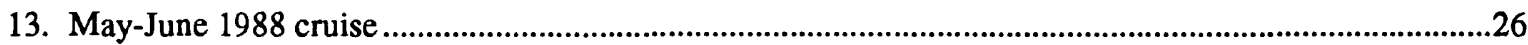

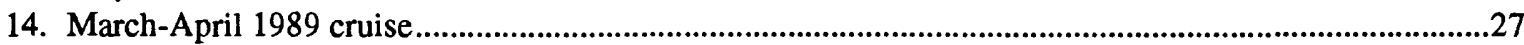

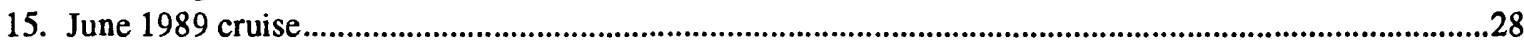

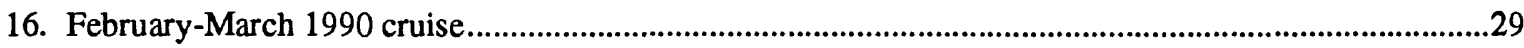

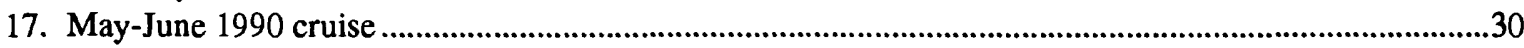

18-24. Concentration data for dissolved trace-elements in water samples collected from the Mississippi River and some of its tributaries during the:

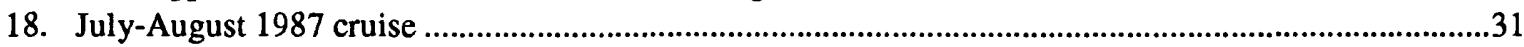

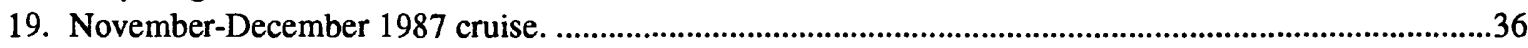

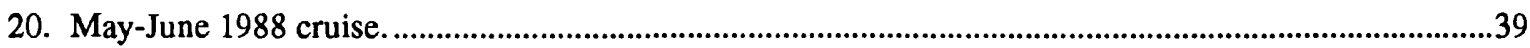

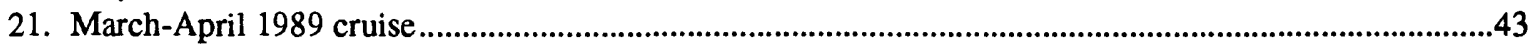

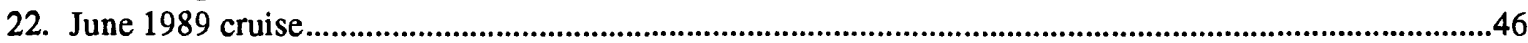




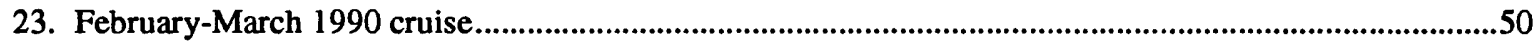

24. May-June 1990 cruise ............................................................................................................................53

25-31. Concentration data for dissolved major elements in water samples collected from

the Mississippi River and some of its tributaries during the:

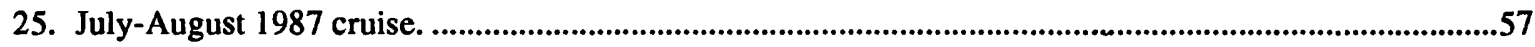

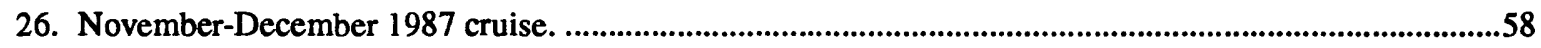

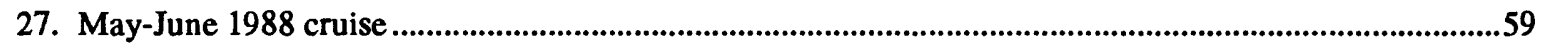

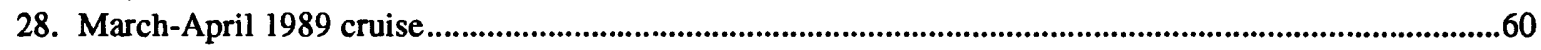

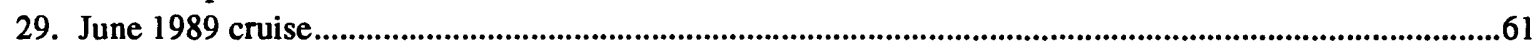

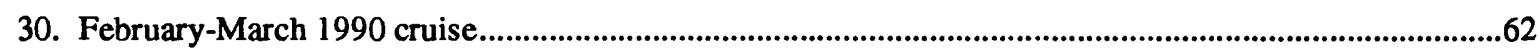

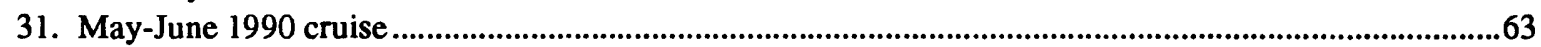

32-34. Concentration data for dissolved chloride, sulfate, and nitrate (as nitrogen) in water samples

collected from the Mississippi River and some of its tributaries during the:

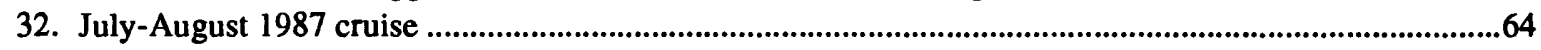

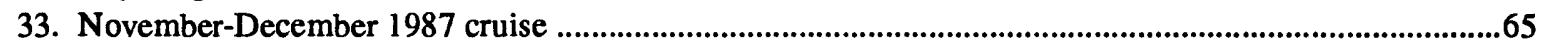

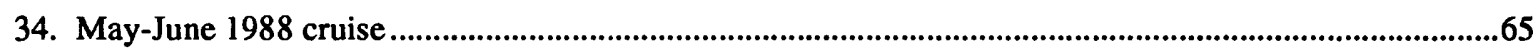

35-36. Concentration data for dissolved chloride and sulfate in water samples collected from the

Mississippi River and some of its tributaries during the

35. March-April 1989 cruise................................................................................................................................66

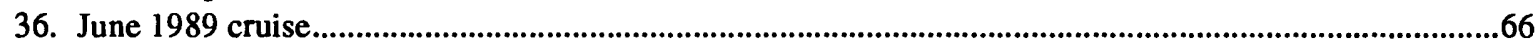

37-38. Concentration data for dissolved chloride, sulfate, and nitrate (as nitrogen) in water samples

collected from the Mississippi River and some of its tributaries during the:

37. February-March 1990 cruise.................................................................................................................67

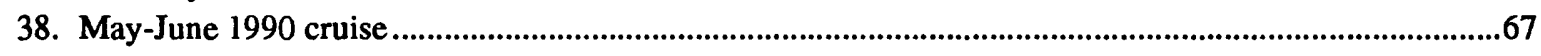

39-45. Concentration data for dissolved bicarbonate and carbonate in water samples

collected from the Mississippi River and some of its tributaries during the:

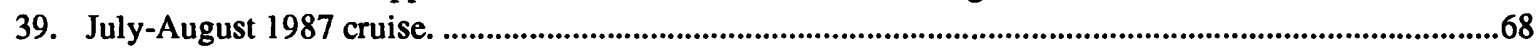

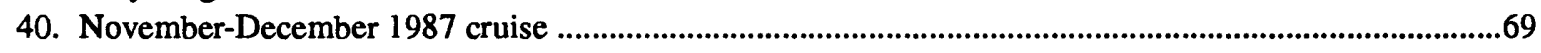

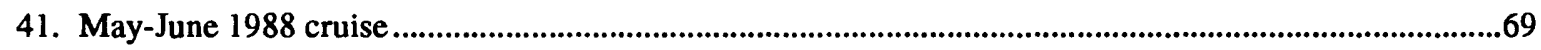

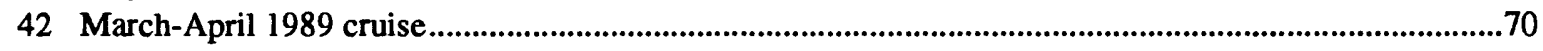

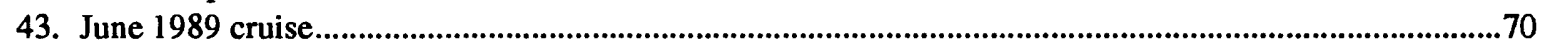

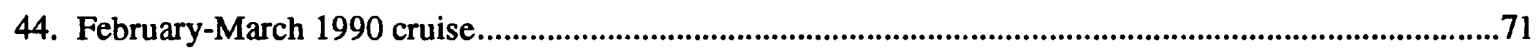

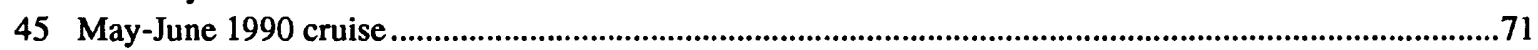

46-52. Discharge and corresponding transport data for dissolved trace elements in water samples collected from the Mississippi River and some of its tributaries during the:

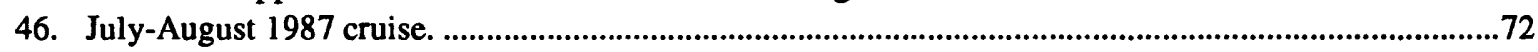

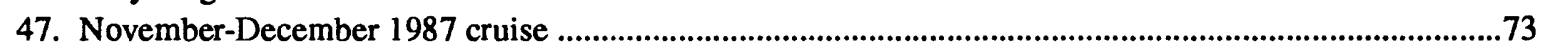

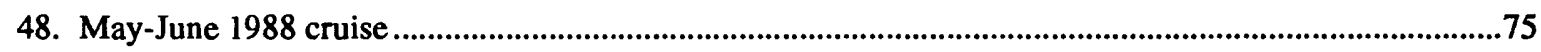

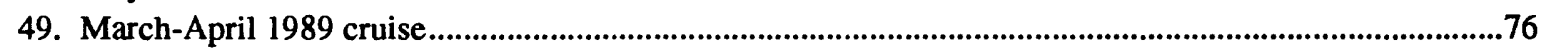

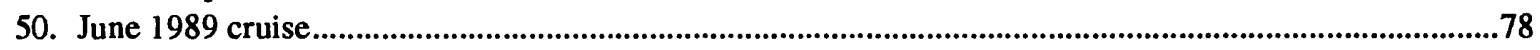

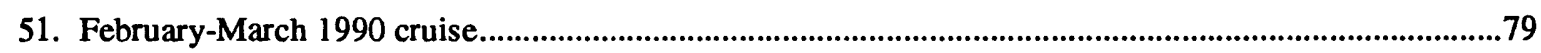

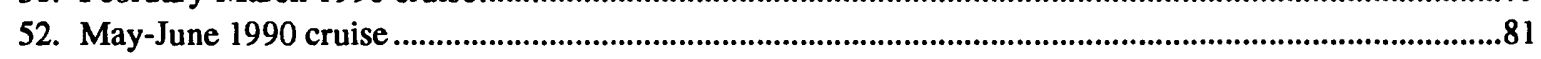

53-59. Discharge and corresponding transport data for dissolved major elements in water samples collected from the Mississippi River and some of its tributaries during the:

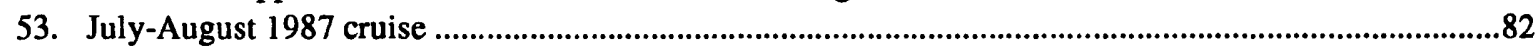

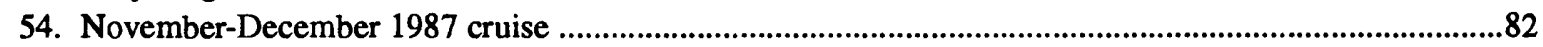

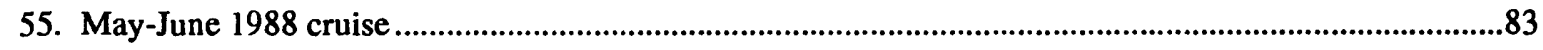

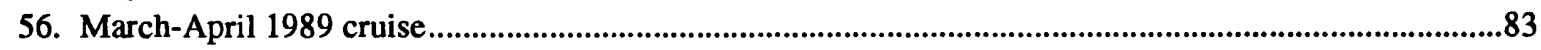

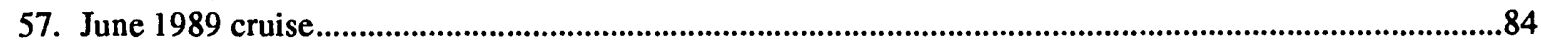

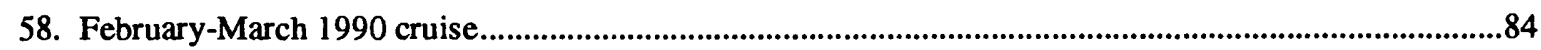

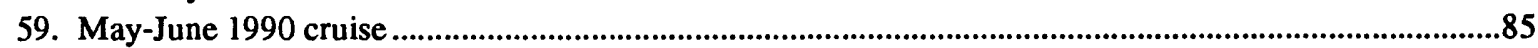


60-66. Discharge and corresponding transport data for dissolved major anions in water samples collected from the Mississippi River and some of its tributaries during the:

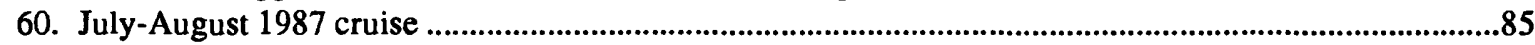

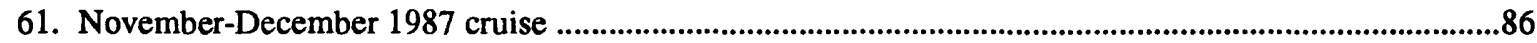

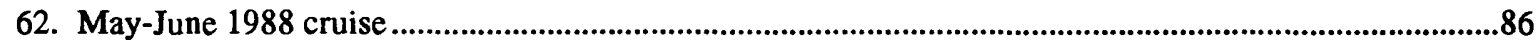

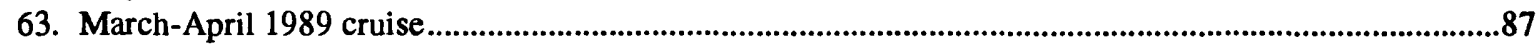

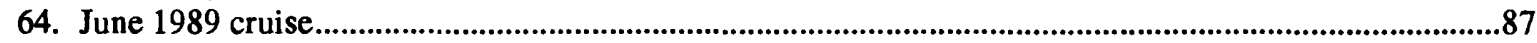

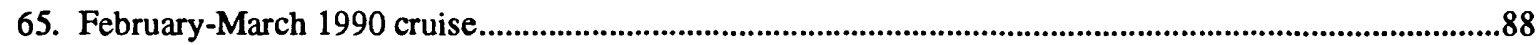

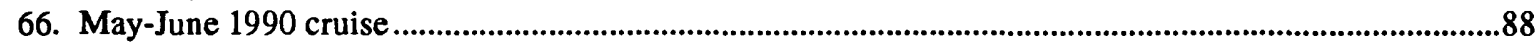

67-73. Concentration data for dissolved trace elements in ultrafilter permeate from water samples collected

from the Mississippi River and some of its tributaries during the:

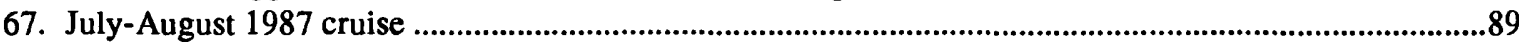

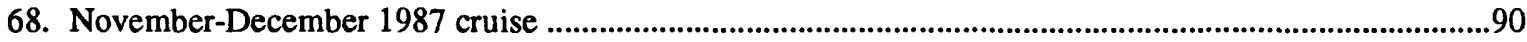

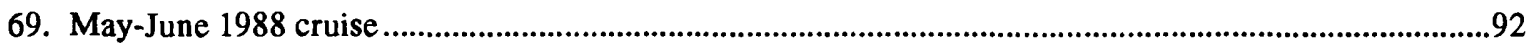

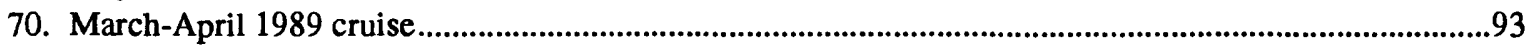

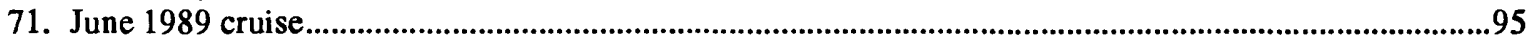

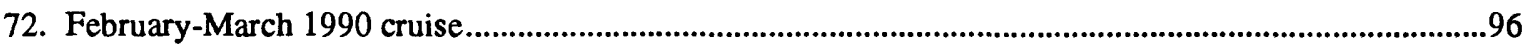

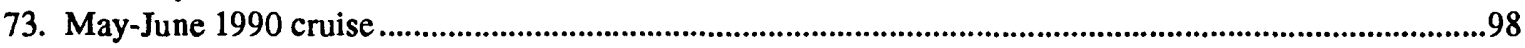

74-80. Concentration data for dissolved major elements in ultrafilter permeate from water samples collected from the Mississippi River and some of its tributaries during the:

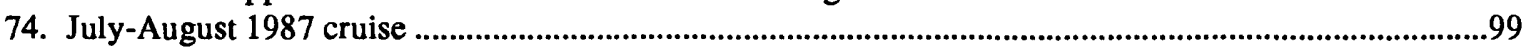

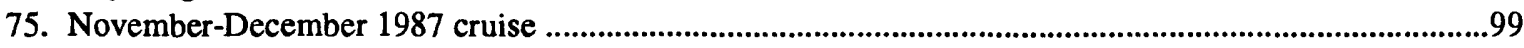

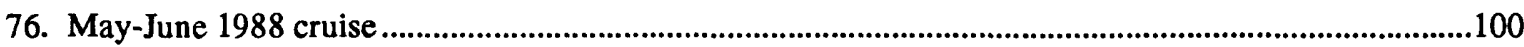

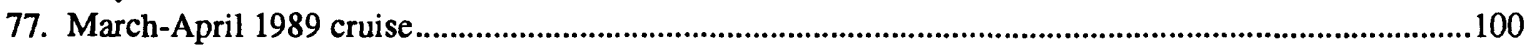

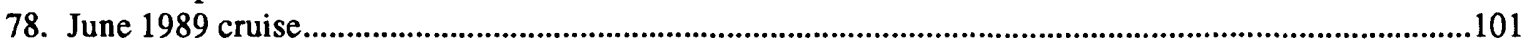

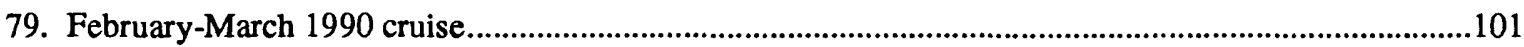

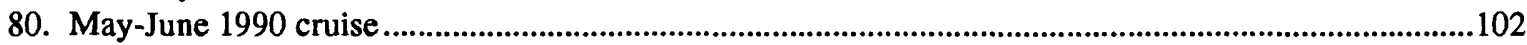

\section{CONVERSION FACTORS and ABBREVIATIONS}

\begin{tabular}{|c|c|c|}
\hline Multiply & By & To obtaln \\
\hline \multicolumn{3}{|c|}{ Length } \\
\hline meter (m) & 0.3048 & foot \\
\hline centimeter $(\mathrm{cm})$ & 2.54 & inch \\
\hline millimeter $(\mathrm{mm})$ & 25.4 & inch \\
\hline micrometer (um) & 400 & inch \\
\hline \multicolumn{3}{|c|}{ Volume } \\
\hline milliliter $(\mathrm{mL})$ & $\underline{0.03382}$ & ounce, fluid \\
\hline liter (L) & 0.2642 & gallon \\
\hline \multicolumn{3}{|c|}{ Flow } \\
\hline cubic meter per second $\left(\mathrm{m}^{3} / \mathrm{s}\right)$ & 0.02832 & cubic foot per second \\
\hline \multicolumn{3}{|c|}{ Mass } \\
\hline microgram $(\mu \mathrm{g})$ & 0.00000003527 & ounce, avoirdupois \\
\hline milligram (mg) & 0.00003527 & ounce. avoirdupois \\
\hline kilogram $(\mathbf{k g})$ & 35.27 & ounce, avoirdupois \\
\hline \multicolumn{3}{|c|}{ Temperature } \\
\hline
\end{tabular}




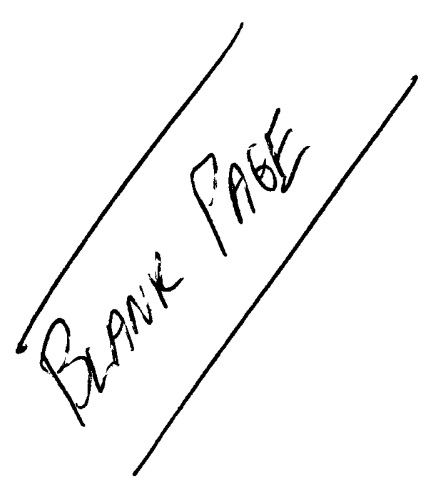

vi 


\title{
CONCENTRATION AND TRANSPORT DATA FOR DISSOLVED INORGANIC CONSTITUENTS IN WATER COLLECTED DURING SEVEN CRUISES ON THE MISSISSIPPI RIVER AND SOME OF ITS TRIBUTARIES, JULY 1987-JUNE 1990
}

\author{
By T.I. Brinton, J.R. Garbarino, D.B. Peart, H.E. Taylor and R.C. Antweiler
}

\begin{abstract}
Concentration and corresponding transport values for selected dissolved constituents were determined for samples collected on the Mississippi River and some of its tributaries at three low-water discharges from July 18, 1987, to June 7, 1988 and four high-water discharges from March 9, 1989, to June 27, 1990. Data were obtained for major elements--calcium, magnesium, potassium, silicon $\left(\mathrm{as} \mathrm{SiO}_{2}\right)$, and sodium; trace elements--aluminum, arsenic, barium, beryllium, boron, cadmium, chromium, cobalt, copper, iron, lead, lithium, manganese, molybdenum, nickel, strontium, thallium, uranium, vanadium, and zinc; alkalinity, and major anions--chloride, sulfate, and nitrate as nitrogen--for selected cruises. Analytical techniques used were inductively coupled plasmaatomic emission spectrometry, inductively coupled plasma-mass spectrometry and ion chromatography. Protocols were designed and implemented to separate suspended sediment from whole water, ensure sample preservation, and estimate sample collection and sample processing errors.
\end{abstract}

\section{INTRODUCTION}

The Mississippi River and its tributaries comprise the largest river system in the United States (fig. 1). It drains both the heavily industrialized urban areas to the east and agricultural rural areas to the west. In addition to being one of the major sources of chemicals and sediment discharged into the Gulf of Mexico, the system also provides the primary drinking-water supply for many communities.

This report presents data on the occurrence and distribution of dissolved constituents in the river system. The distribution, movement, and storage of contaminants in the river system have a substantial effect on the ecology, environmental chemistry, and utilization of the resource. A multidisciplinary research investigation has been undertaken by the U.S. Geological Survey to study the water quality of the river system. This investigation includes the study of the water chemistry as related to inorganic contaminants and their interaction with river sediments. Both naturally occurring and anthropogenic trace elements are found in the river system. These trace elements principally originate from mineral deposits, drainage from mining activities, industrial manufacturing and processing, and municipal wastes. Trace elements are in equilibrium among soluble species, suspendedparticulate matter, and bed sediments, including insoluble precipitates. The soluble species range from aqueous to organic complexes that are often $\mathrm{pH}$ dependent. This partitioning and interaction plays a significant role in the transport and storage of trace elements in the river system.

Numerous references report concentrations of selected trace elements and chloride, sulfate, nitrate and bicarbonate ions in the Mississippi River (Taylor and others, 1990; T.I. Brinton, R.C. Antweiler and H.E. Taylor, unpub. data, 1992). Other reports describe data on a number of elements collected during long-term monitoring programs (Briggs and Ficke, 1978; Smith and others, 1987) or during a specific study on a limited reach of the river (Everett, 1971; Wells, 1980; Shiller and Boyle, 1983; 1987; Trefry and others, 1986; Taylor and Shiller, 1995; Taylor and others, 1995). 
This report provides concentration data on several dissolved constituents in the Mississippi River and some of its tributaries on a geographic and seasonal basis, as a foundation to the detailed study of the chemical and physical partitioning of trace elements with sediments. This design not only provides information on the geographical variations due to tributary inflow and point sources, but also provides some insight on the effects due to variations in season and river stage. Data in this report are from samples collected during three low-water cruises from July 18, 1987, to June 7, 1988, and four high-water cruises from March 8, 1989, to June 27, 1990.

Special thanks are given to the research vessel ACADIANA crew, Lee Black, Wilton Delaune, Chuck Guidry, Craig LeBoeuf, Ken Oliver, Wayne Simoneaux, and Steve Rabalais. Gratitude is expressed to the many colleagues who assisted in collecting and processing samples and providing logistical support including Daphne Frilot, Lois Koehnken, Tom Kraemer,Dick Martin, Brent McKee, Alan Shiller, Dick Tagg, Tim Willoughby, Ellen Axtmann, Pat Brown, Wes Campbell, Jerry Leenheer, Tom Leiker, Debby Martin, Robert Meade, John Moody, Ted Noyes, Wilfred Pereira, James Ranville, Terry Rees, Colleen Rostad, James Seeley, Robert Stallard, and Herbert Stevens. Appreciation is expressed to Heidi Hayes, and David Roth for their assistance in completing the laboratory analyses and compiling data for this report.

\section{SAMPLE COLLECTION}

Sampling was conducted from the research vessel ACADIANA at predetermined sites on the Mississippi River and some of its tributaries--the Illinois, Missouri, Ohio, White, Arkansas, and Yazoo Rivers. During one cruise, additional sites were sampled on the Ohio River and its major tributaries, the Wabash, Cumberland, and Tennessee Rivers. Sites were chosen at selected locations along the Mississippi River and at locations below the confluence with tributaries. The sampling strategy used the Lagrangian principle (Meade and others, 1985), in which an attempt was made to follow a fixed parcel of water downstream and sample it at each of the sites in a sequential fashion. The use of this sampling technique is described in more detail by Meade and others (1985); Meade and Stevens (1990); Moody and Meade (1992, 1993); Moody, (1992), and Leenheer and others, (1989). Sampling sites are listed in table 1 and relative locations of the sampling sites in the river system are shown in figure 1 .

A previously described collapsible-bag sampler (Stevens and others, 1980; Nordin and others, 1983; and Meade, 1985) was used to collect depth-integrated samples from 10 to 40 verticals at each sampling site (see Moody and Meade, 1992, for more details.) Samples from alternate verticals were combined into two composite samples (composite A and composite B) which were analyzed independently to evaluate sampling precision. Approximately $100 \mathrm{~L}$ of total composited sample was collected at each site. Teflon bags were used in the bag sampler to minimize sample contamination and to facilitate quantitative transfer of sample and suspended material. Extreme care was used to minimize any possible metal contamination by using Teflon, high-density polyethylene, or Teflon-coated stainless-steel sample-processing equipment. Talc-free polyethylene, polyvinyl chloride, or Teflon gloves were used during the entire sample handling and processing procedure.

In addition to the depth-integrated composite samples, a grab sample was collected about $30 \mathrm{~cm}$ below the surface at the centroid-of-flow using a 1-L polyethylene bottle mounted on the end of a 4-m fiberglass pole. This sample provided an indication of how well mixed the water at the sampling site was and the degree of importance for depth-integrated sample collection at each site.

\section{SAMPLE PROCESSING}

Composited samples were passed through a $63-\mu \mathrm{m}$ nickel screen to remove sand-size suspended material and miscellaneous debris. Field blanks using deionized water (Nanopure) indicate that the nickel screen contributes negligible contamination to trace-element concentrations. Both composite A and composite B samples, made up of water, and particulate matter smaller than $63 \mu \mathrm{m}$, were representatively split into subsamples using a Teflon coated stainless-steel churn splitter. Individual aliquots were taken from the splitter for trace elements, major anions, and alkalinity determinations. 
A Teflon or plastic vacuum filter apparatus, similar to the one shown in figure 2 , was used to remove particulate matter greater than $0.40 \mu \mathrm{m}$ from the trace-element subsample. A precise protocol was used for the filtration: (1) The filter funnel was thoroughly cleaned and rinsed with deionized water (Nanopure); (2) a new $0.40-\mu \mathrm{m}$ pore size $47-\mathrm{mm}$-diameter Nuclepore polycarbonate-membrane filter was placed on the filter support and precleaned by drawing $50 \mathrm{~mL}$ of 0.1 percent (volume/volume) ultrapure nitric acid rinse solution through the filter into a waste bottle; (3) the filter was then rinsed by drawing $100 \mathrm{~mL}$ of deionized water (Nanopure) through it into a waste bottle; (4) about $25 \mathrm{~mL}$ of the shaken composited subsample was then filtered to prerinse the sample bottle and also effectively preload the filter with particulate; (5) the balance of the subsample (about $225 \mathrm{~mL}$ ) was filtered into the sample bottle; and (6) the filtered sample was preserved with the addition of $1 \mathrm{~mL}$ of concentrated ultrapure nitric acid (or to a pH less than 2) using a Teflon dispensing bottle (shown in fig. 3). Only one filter membrane was used for the entire aliquot. The grab sample was processed identically.

The major anion and alkalinity subsamples were immediately chilled to approximately $4^{\circ} \mathrm{C}$, and no preservatives were added. These raw-water samples were transported on ice in coolers to the laboratory. Subsamples for major anions were filtered through a $0.45-\mu \mathrm{m}$ pore size, 25 -mm-diameter polysulfone-membrane syringe filter and analyzed. Subsamples for alkalinity were decanted from the chilled, settled samples and analyzed.

Ordinarily, a separate nutrient sample is split, processed, preserved, and analyzed for nitrate. The conventional procedure of adding mercuric chloride as an antibacterial preservative was considered inappropriate because of possible cross-contamination of other samples. Consequently, nitrate ion results were measured on the major anion subsample as part of the ion chromatographic procedure. Although the reported nitrate data are considered accurate, results are tabulated primarily for informational purposes and should not be considered definitive.

The remaining portions of composite $\mathrm{A}$ and $\mathrm{B}$ were combined and bulk processed, as described by Leenheer and others (1989) and Rees and others (1991), to separate suspended particulate matter into specific particle size fractions. The greater than 4- $\mu \mathrm{m}$ particulate was first removed using a continuous-flow Teflon-coated centrifuge (Sharples, model AS-12). The effluent from the centrifuge was then processed through a tangential-flow ultrafilter to remove particulates larger than $0.005 \mu \mathrm{m}$. The separated particulate fractions from the centrifuge and ultrafilter were retained for characterization by sequential extractions and chemical analysis. The membranes used in the ultrafilter were made of deacetylated cellulose. Trace-element adsorption studies were done and it was determined that no significant adsorption occurred on this membrane material (Leenheer and others, 1989). The ultrafilter system, without membrane filter plates in place, was cleaned by pumping $10 \mathrm{~L}$ of deionized water (Nanopure) through the system between each sample. To ensure that no contamination from the filter membranes occurred, a dilute ultrapure nitric acid solution ( $\mathrm{pH}$ 3.0-3.5) was passed through the filters and apparatus before processing the sample. A stack of five new filter plates was typically used at each site to provide suitable processing times and to eliminate carryover contamination between sites. Effluent from the ultrafilter, termed "ultrafilter permeate," was subsampled for trace elements and acidified as described above for the composites. Analytical results for the permeate sample provided an indication of the fraction of trace elements, particularly iron, manganese, and aluminum, that are associated with colloidal-sized material (Kennedy and others, 1974).

\section{QUALITY CONTROL}

The quality of the analytical results was controlled by collecting numerous field blanks and analyzing certified reference standards extensively along with the analysis of the samples. Field blanks were used to identify possible sources of contamination to samples during their collection and processing. Reference standards were used to monitor the accuracy of the analyses and the performance of laboratory instrumentation.

Two or three times during each sampling cruise, a series of field blanks were collected by processing about 30 to $60 \mathrm{~L}$ of shipboard deionized water (Nanopure) through the entire sample collection, subsampling, and sample-processing procedures in an effort to identify possible sources of sample contamination. Prior to 
processing the field blank, a sample of the shipboard deionized water was collected. When processing the deionized water through the entire procedure, aliquots were taken at various steps. Samples were collected for trace-element analysis that represented water processed through the bag sampler using a Teflon bag, the nickel sieve and the glass $2,000 \mathrm{~mL}$ graduated cylinder used for collection efficiency measurements, and the 8 and 20 -L Teflon-coated churn splitters. The remainder of the deionized water in the splitters was processed through the centrifuge followed by the tangential-flow ultrafilter with new filter plates. Samples of centrifuge effluent and ultrafilter permeate also were taken. Finally, deionized water was filtered through a Nuclepore filter to obtain a filter blank. All the field blank samples were preserved following the same protocols as their corresponding sample types. Major- and trace-element results for the field blanks from the subject cruises are tabulated in tables 2-10. Field blanks for major anions consisted of collecting shipboard deionized water, results of which indicated negligible contamination.

The accuracy of the sample data was ensured by analyzing 1 to 3 standard reference materials, periodically, within a set of unknown samples. Reference standards routinely comprised about 15 percent of an analytical run, or 1 in every 7. Twenty-four unknown samples were analyzed after every instrument calibration to minimize effects of instrumental drift. Unknown samples were reanalyzed whenever the experimental reference standard mean was greater than two standard deviations of the certified reference standard most probable value. Data for one of the reference standards used for quality assurance for individual cruises are listed in tables 11-17. The average and standard deviations are based on the sample population given and represent the accuracy and precision throughout the analysis of the entire set of samples from a given cruise.

Sampling precision can be estimated by comparing results obtained for composite A and composite B from data listed in tables 18 to 45 . Calculations show good correlation (correlation coefficient of .984) between results for composite $A$ and $B$ for calcium, which is a typical element ranging in concentration from 20 to $70 \mathrm{mg} / \mathrm{L}$, from the May-June 1988 cruise. Calculations for lead, a typical element ranging in concentration from 0.1 to $0.5 \mu \mathrm{g} / \mathrm{L}$, from the May-June 1988 cruise show similar results. The sampling precision for other elements and sampling periods show equivalent relations.

\section{ANALYTICAL METHODS}

Trace elements were determined by a combination of analytical techniques including inductively coupled plasma-mass spectrometry (ICP-MS), inductively coupled plasma-atomic emission spectrometry (ICP-AES), and flame atomic absorption spectrometry (F-AAS). A Perkin Elmer-Sciex ICP-MS was configured and optimized for trace-element analyses as previously described (Taylor and Garbarino, 1991, and Garbarino and Taylor, 1992). Two basic types of analyses were performed using this technique: (1) A semiquantitative survey analysis detecting essentially all stable isotopes at detection limits of less than $0.1 \mu \mathrm{g} / \mathrm{L}$ and (2) individual element quantitative analysis with appropriate interference corrections. The analytes determined quantitatively were aluminum, arsenic, barium, beryllium, boron, cadmium, chromium, cobalt, copper, lead, lithium, manganese, molybdenum, nickel, thallium, uranium, vanadium, and zinc. A complete description of the analytical method, a listing of detection limits, and accuracy and precision data are provided by Garbarino and Taylor (1992).

Other elements were determined using a Jarrell-Ash Atomcomp 975 inductively coupled argon plasmaatomic emission spectrometer. The analytical system, methodology, detection limits, and accuracy and precision data for this method are described by Garbarino and Taylor $(1979,1980)$ and Skougstad and others (1979). The analytes determined were calcium, magnesium, sodium, strontium, silicon $\left(\mathrm{as}_{\mathrm{SiO}}\right)$, and iron. Potassium was determined by flame-atomic absorption spectrometry (F-AAS) using an Instrumentation Laboratory, Model 751 instrument and the method described by Skougstad and others (1979).

Major anion concentrations (chloride, sulfate, and nitrate), which are used to describe general water chemistry, were determined by ion chromatographic techniques (Hedley and Fishman, 1982; T.I. Brinton, R. C. Antweiler and H.E. Taylor, unpub. data, 1992). Alkalinity, as bicarbonate, was determined using an automated Radiometer titrator and calculated from the $\mathrm{pH}$ data (Skougstad and others, 1979). 


\section{ANALYTE CONCENTRATION AND TRANSPORT DATA}

The data obtained from the analyses of water samples collected from the Mississippi River and some of its tributaries are listed in tables 18-80. All the samples were analyzed in a random fashion, the reagent was blank subtracted, and spectral background corrected. Results that were less than the detection limit are identified by the less-than symbol (<), preceding the detection limit value. Results for major- and trace-elements represent a mean based on two to five replicate analyses. Results for major anions represent a single analysis. Analytes that were not determined for a particular sample are identified by --, and transport data for which the corresponding concentration was less than the detection limit are identified by *.

Dissolved trace-element concentrations (aluminum, arsenic, barium, beryllium, boron, cadmium, cobalt, chromium, copper, iron, lead, lithium, manganese, molybdenum, nickel, strontium, thallium, uranium, vanadium, and zinc) are in tables 18-24. Dissolved major-element concentrations (calcium, magnesium, potassium, sodium, silicon as $\mathrm{SiO}_{2}$ are in tables 25-31. Dissolved major anion [chloride, sulfate, and nitrate as nitrogen] concentrations are in tables 32-38. Dissolved carbonate, and bicarbonate concentrations are in tables 39-45.

Transport values were calculated by multiplying the analyte concentration by the measured water discharge and a unit conversion factor $(0.0864$ if concentration is reported in $\mu \mathrm{g} / \mathrm{L}$ and 86.4 if concentration is reported in $\mathrm{mg} / \mathrm{L}$ ). All transport values are in units of kilograms per day $(\mathrm{kg} / \mathrm{d})$. Water discharges were measured during the cruises and are reported by Moody and Meade (1992, and 1993), and the corresponding calculated trace-element transport data for sampling sites of the Mississippi River and some of its tributaries for every cruise are listed in tables 46-52. Major-element transport data are listed in tables 53-59. Major-anion transport data are in tables 6066.

Dissolved trace-element concentrations for ultrafilter permeate aliquots for every cruise are in tables 67-73. Dissolved major-element concentrations for ultrafilter permeate aliquots are in tables 74-80. Permeate results for iron, manganese, and aluminum reflect the truly dissolved concentration because of the removal of the colloidal fractions greater than $0.005 \mu \mathrm{m}$.

\section{REFERENCES}

Briggs, J.C., and J.F. Ficke, 1978, Quality of rivers of the United States, 1975 water year: U.S. Geological Survey Open-File Report 78-200, 436 p.

Everett, D.E., 1971, Hydrologic and quality characteristics of the lower Mississippi River, Louisiana: Louisiana Department of Public Works Technical Report 5, 48 p.

Garbarino, J.R., and Taylor, H.E., 1979, An inductive-coupled plasma atomic-emission spectrometric method for routine water quality testing: Applied Spectroscopy, v. 33, p. 220-226.

Garbarino, J.R., and Taylor, H.E., 1980, A Babington-type nebulizer for the use in the analysis of natural water samples by inductively coupled plasma spectrometry: Applied Spectroscopy, v. 34, p. 393-399.

Garbarino, J.R., and Taylor, H.E., 1992, Inductively coupled plasma-mass spectrometric method for the determination of dissolved trace elements in water: U.S. Geological Survey Open-File Report 94-358.

Hedley, A.G., and Fishman, M.J., 1982, Automation of an ion chromatograph for precipitation analysis with computerization data reduction: U.S. Geological Survey Water-Resources Investigations Report 81-78, 38 p.

Kennedy, V.C., Jones, B.F., and Zellweger, G.W., 1974, Filter pore-size effects on the analysis of As, Fe, Mn, and Ti in water: Water Resources Research, v. 10, p. 785-790. 
Leenheer, J.A., Meade, R.H., Taylor, H.E., and Pereira, W.E., 1989, Sampling, fractionation, and dewatering of suspended sediment from the Mississippi River for geochemical and trace contaminant analysis, in Mallard, G.E., ed., U.S. Geological Survey Toxic Substances Hydrology Program,--Proceedings of the technical meeting, Phoenix, Arizona, September 26-30, 1988: U.S. Geological Survey Water- Resources Investigations Report 88-4220, p. 501-511.

Meade, R.H., 1985, Suspended sediment in the Amazon River and its tributaries in Brazil during 1982-84: U.S. Geological Survey Open-File Report 85-492, 34 p.

Meade, R.H., Dunne, T., Richey, J.E. U. de M. Santos, and Salati, E., 1985, Storage and remobilization of suspended sediment in the lower Amazon River of Brazil: Science, v. 228, p. 488-490.

Meade, R.H., and Stevens, H.H., 1990, Strategies and equipment for sampling suspended sediment and associated toxic chemicals in large rivers--with emphasis on the Mississippi River: The Science of the Total Environment, v. 97/98, p. 125-136.

Moody, J.A., 1992, Evaluation of the Lagrangian scheme for sampling the Mississippi River during 1987-90: U.S. Geological Survey Open-File Report 93-4042, 31 p.

Moody, J.A., and Meade, R.H., 1992, Hydrologic and sedimentologic data collected during three cruises at low water on the Mississippi River and some of its tributaries, July 1987-June 1988: U.S. Geological Survey Open-File Report 91-485, 143 p.

Moody, J.A. and Meade, R. H., 1993, Hydrologic and sedimentologic data collected during four cruises at high water on the Mississippi River and some of its tributaries, March 1989 - June 1990: U.S. Geological Survey Open-File Report 92-651, 227 p.

Nordin, C.F., Jr., Cranston, C.C., and Mejia-B., A., 1983, New technology for measuring water and suspendedsediment discharge of large rivers, in Proceedings of the International Symposium on River Sedimentation, 2d, Nanjing, China, October 11-16, 1983: Beijing, Water Resources and Electric Power Press, theme E, paper E20, p. 1145-1158.

Rees, T.F., Leenheer, J.A., and Ranville, J.F., 1991, Use of a single-bowl continuous-flow centrifuge for dewatering suspended sediment--effect on sediment physical and chemical characteristics: Hydrological Processes, v. 5, p. 210-214.

Shiller, A.M. and Boyle, E., 1983, Trace metals in the plume of the Mississippi River; EOS, v. 64, p. 1021.

Shiller, A.M. and Boyle, E., 1987, Variability of dissolved trace metals in the Mississippi River: Geochimica et Cosmochimica Acta, v. 51, p. 3273-3277.

Skougstad, M.W., Fishman, M.J., Friedman, L.C., Erdman, D.E., and Duncan, S.S., 1979, Methods for the determination of inorganic substances in water and fluvial sediments: U.S. Geological Survey Techniques of Water Resources Investigations, book 5, chap. A1, p. 229.

Smith, R.A., Alexander, R.B., and Wolman, M.G., 1987, Water-quality trends in the Nation's rivers: Science, v. 235, p. 1607-1615.

Stevens, H.H., Jr., Lutz, G.A., and Hubbell, D.W., 1980, Collapsible-bag suspended-sediment sampler: American Society of Civil Engineers Proceedings, Hydraulic Division Journal, v. 166, no. HY4, p. 611-616.

Taylor, H.E., and Garbarino, J.R., 1991, The measurement of trace metals in water resource monitoring samples by inductively coupled plasma-mass spectrometry: Spectrochimica Acta Reviews, v. 14, p. 33-44. 
Taylor, H.E., Garbarino, J.R., and Brinton, T.I., 1990, The occurrence and distribution of trace metals in the Mississippi River and its tributaries: The Science of the Total Environment, v. 97/98, p. 369-384.

Taylor, H.E. and Shiller, A.M., 1995, Mississippi River methods comparison Study: Implications for water quality monitoring of dissolved trace elements: Environmental Science and Technology, v. 29, no. 5, p. 1313-1317.

Taylor, H.E., Shiller, A.M., Garbarino, J.R., and Brinton, T.I., 1995, Intercomparison experiments on dissolved trace-metal data from the Mississippi River and some of its tributaries, 1989-1990: U.S. Geological Survey Open-File Report 93-628, 28 p.

Trefry, J.H., Nelson, T.A., Trocine, R.P, Metz, S., and Vetler, T., 1986, Trace metal fluxes through the Mississippi River delta system, in Kullenberg, G., ed, Contaminant Fluxes Through the Coastal Zone: Rapp. P.-v. Reun. Const. Int. Explor. Mer, 186, p. 277-288.

Wells, F.C., 1980, Hydrology and water quality of the lower Mississippi River: Louisiana Department of Public Works Technical Report 21, 83 p. 


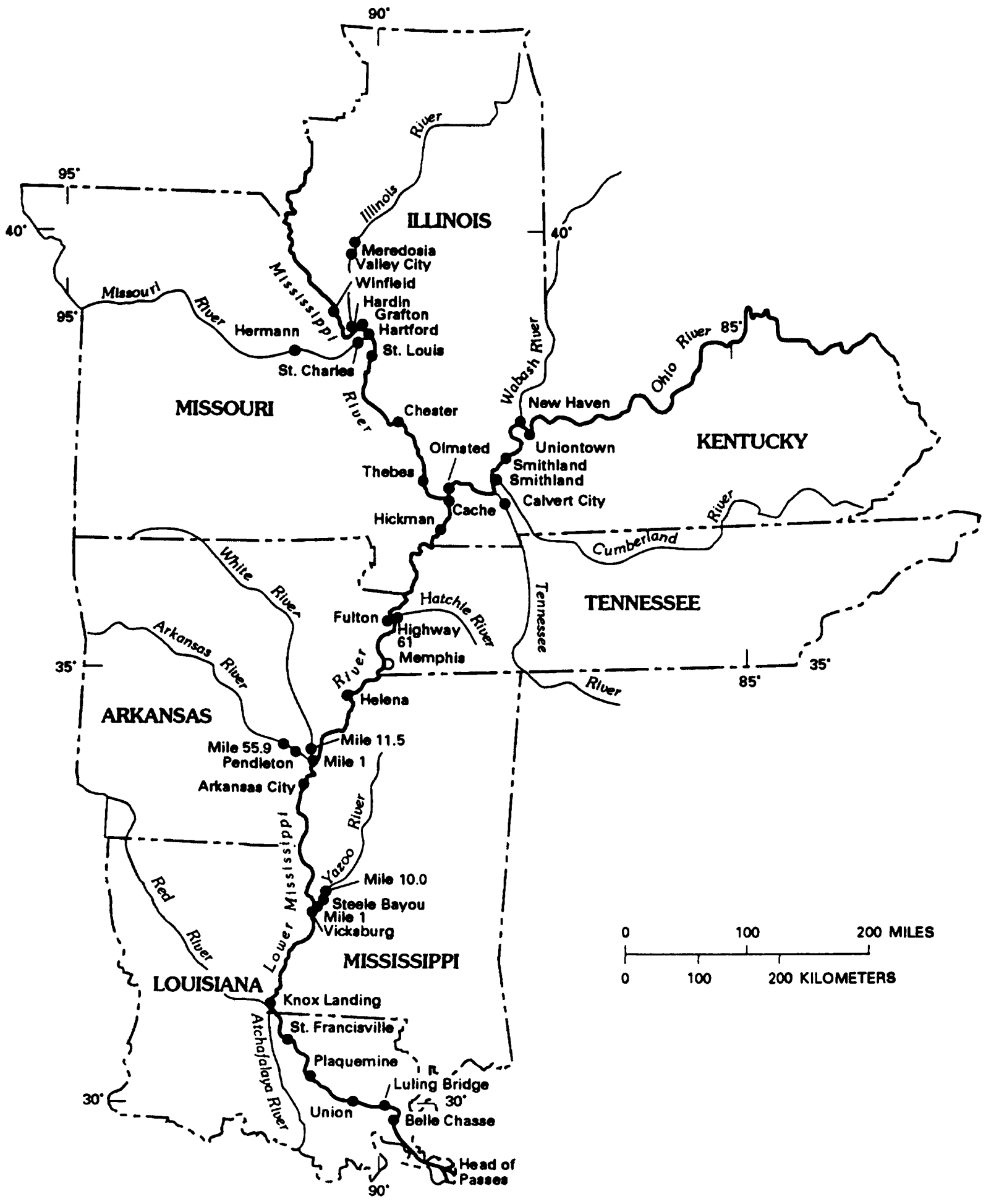

GULF OF MEXICO

Figure 1. Map showing the Mississippi River and some of its tributaries. 


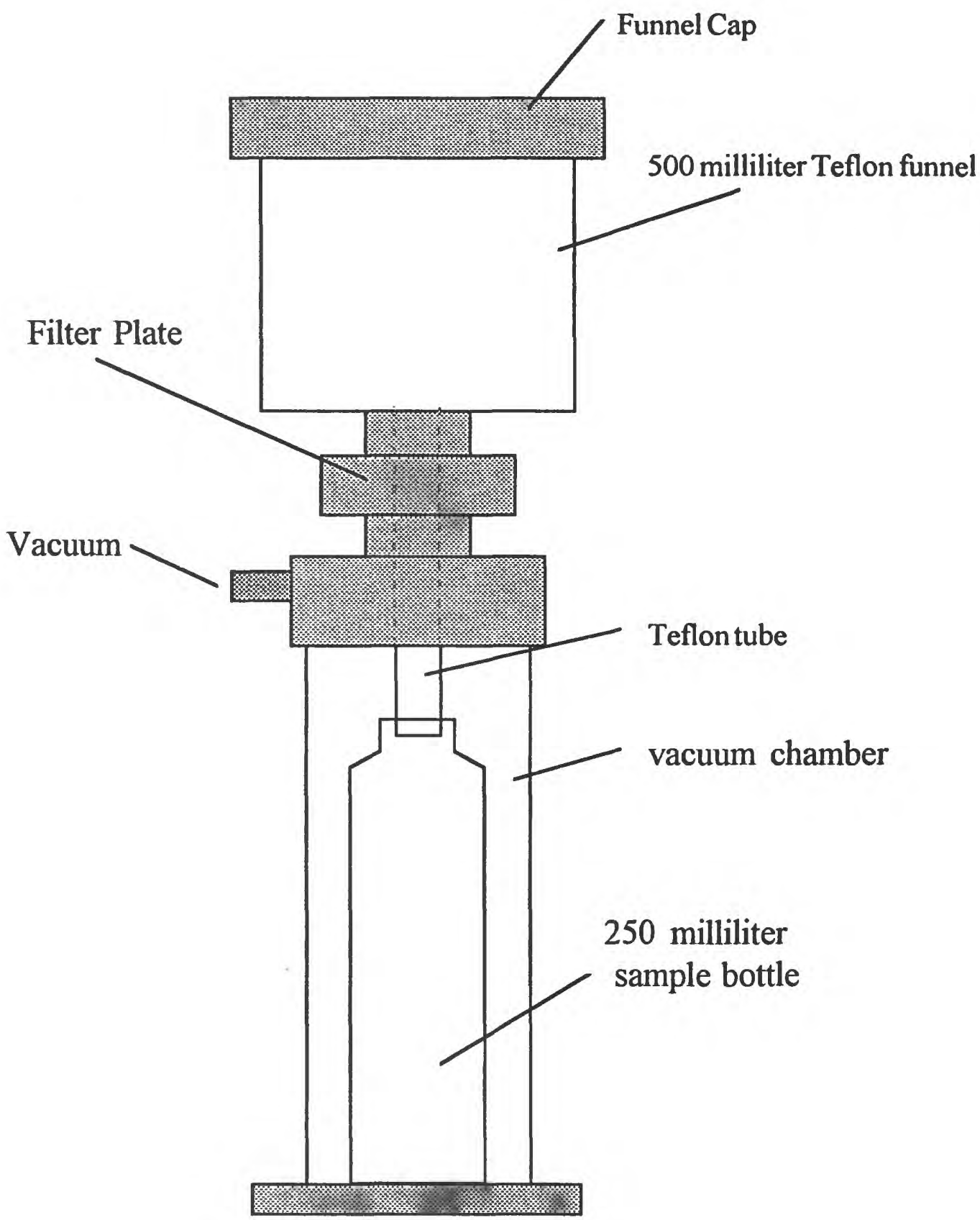

Figure 2. Schematic diagram of filter apparatus used for filtration of trace-element samples. 


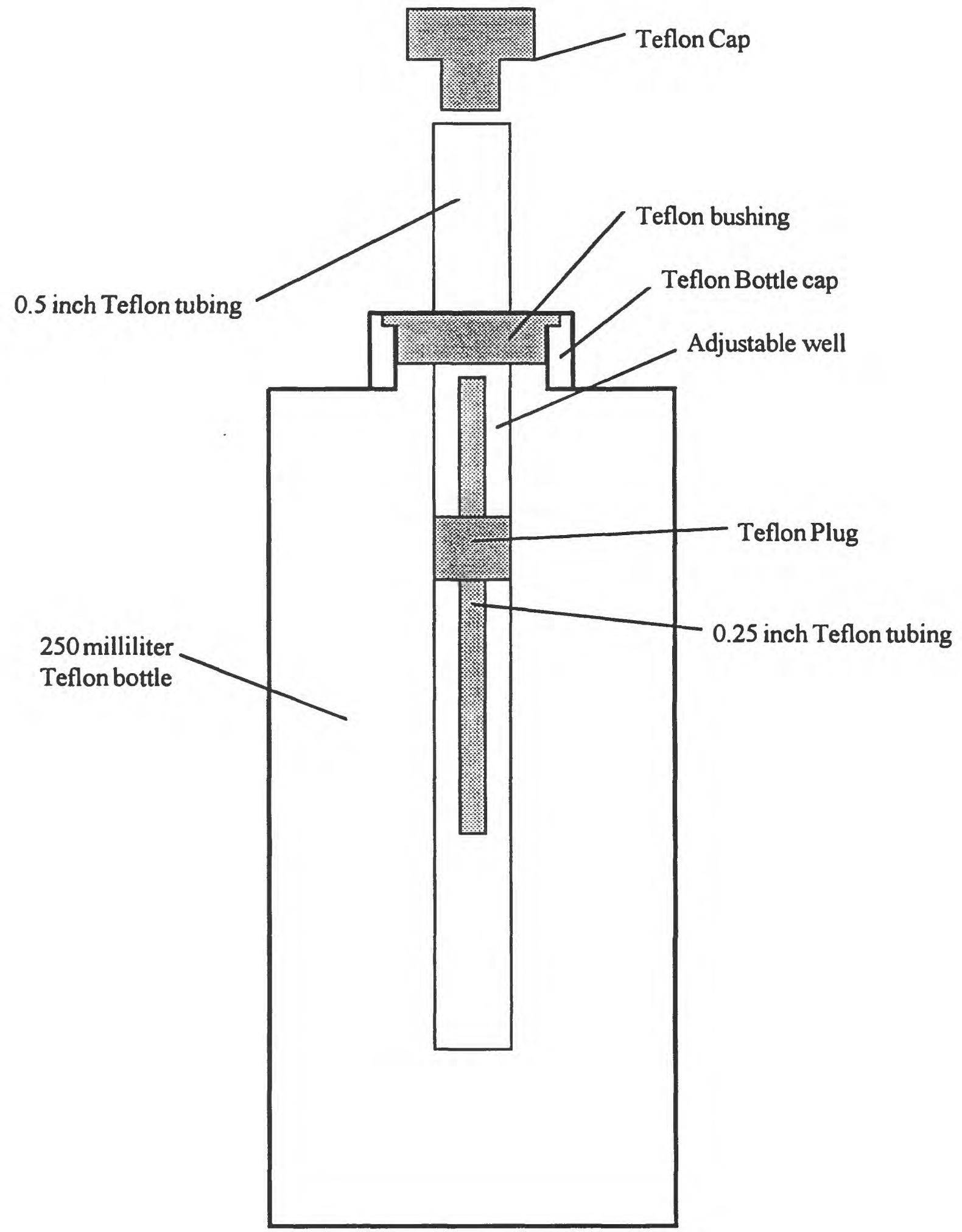

Figure 3. Schematic diagram of nitric acid dispensing bottle. 


\section{Table 1.--Sampling sites on the Mississippi River and some of its tributaries}

[UM, Upper Mississippi River miles measured upriver from confluence with Ohio River, IL, Illinois River miles measured upriver from the confluence with Mississippi River (UM 218.0); MO, Missouri River miles measured upriver from the confluence with Mississippi River (UM 195.3); OH, Ohio River miles measured downriver from Pittsburgh, Pa. Ohio-Mississippi confluence is OH 981.5 and LM 953.8; WA, Wabash River miles measured upriver from confluence with Ohio River $(\mathrm{OH} 848.0)$; $\mathrm{CU}$, Cumberland River miles measured upriver from confluence with Ohio River (OH 923.2); TE, Tennessee River miles measured upriver from confluence with Ohio River (OH 935.5); LM, Lower Mississippi River miles measured upriver from Head of Passes, La.; WH, White River miles measured upriver from confluence with Mississippi River (LM 598.8); AR, Arkansas River miles measured upriver from confluence with Mississippi River (LM 581.5); YZ, Yazoo River miles measured upriver from confluence with Mississippi River (LM 437.2); OR, Old River Outflow Channel miles measured downriver from the Old River Control structure (LM 314.5)]

\begin{tabular}{|c|c|c|c|c|c|c|c|c|}
\hline Sampling site & $\begin{array}{l}\text { River } \\
\text { mile }\end{array}$ & $\begin{array}{c}\text { July- } \\
\text { August } \\
1987 \\
\end{array}$ & $\begin{array}{c}\text { November- } \\
\text { December } \\
1987\end{array}$ & $\begin{array}{l}\text { May- } \\
\text { June } \\
1988 \\
\end{array}$ & $\begin{array}{c}\text { March- } \\
\text { April } \\
1989 \\
\end{array}$ & $\begin{array}{l}\text { June } \\
1989 \\
\end{array}$ & $\begin{array}{c}\text { February- } \\
\text { March } \\
1990 \\
\end{array}$ & $\begin{array}{l}\text { May- } \\
\text { June } \\
1990 \\
\end{array}$ \\
\hline Mississippi River near Winfield, Mo. & UM 239.2 & $\mathrm{X}$ & $\mathrm{X}$ & $\mathrm{X}$ & $\mathrm{X}$ & $\mathrm{X}$ & & \\
\hline Illinois River below Meredosia, Ill. & IL 67.2 & $\mathrm{x}$ & $\mathrm{x}$ & $\mathrm{x}$ & & & & \\
\hline Illinois River at Valley City, Ill. & IL 61.0 & & & & & & & $\mathbf{x}$ \\
\hline Illinois River at Hardin, Ill. & IL 21.8 & & & & $\mathrm{X}$ & $x$ & & \\
\hline Mississippi River below Grafton, Ill. & UM 214.6 & & & & & & & $\mathrm{x}$ \\
\hline Mississippi River at Hartford, Ill. & UM 197.7 & $\mathrm{X}$ & & & & & & \\
\hline Missouri River at Hermann, Mo. & MO 97.9 & $\mathrm{x}$ & & $\mathrm{X}$ & $\mathbf{x}$ & $\mathrm{X}$ & & \\
\hline Missouri River at St. Charles, Mo. & MO 28.1 & & $\mathrm{X}$ & & & & & $\mathrm{X}$ \\
\hline Mississippi River at St. Louis, Mo. & UM 179.3 & $\mathrm{x}$ & $\mathrm{X}$ & $\mathrm{x}$ & $\mathrm{x}$ & $x$ & & \\
\hline Mississippi River at Chester, Ill. & UM 108.9 & $\mathrm{x}$ & & & & & & \\
\hline Mississippi River at Thebes, Ill. & UM 43.9 & & $\mathrm{X}$ & $\mathrm{X}$ & $\mathrm{x}$ & $x$ & & 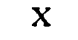 \\
\hline Mississippi River near Cache, Ill. & UM 14.8 & & & & & & $X$ & \\
\hline Ohio River at Uniontown, Ky. & OH 842.4 & & & & & & $\mathrm{x}$ & \\
\hline Wabash River near New Haven, Ill. & WA 13.8 & & & & & & $\mathrm{X}$ & \\
\hline Cumberland River near Smithland, Ky. & CU 6.8 & & & & & & $\mathrm{X}$ & \\
\hline Tennessee River near Calvert City, Ky. & TE 11.1 & & & & & & $\mathrm{X}$ & \\
\hline $\begin{array}{l}\text { Ohio River below Smithland Locks and } \\
\text { Dam, Ill. \& Ky. }\end{array}$ & OH 919.2 & $\mathrm{X}$ & & & & & & \\
\hline Ohio River at Olmsted, Ill. & OH 965.0 & $\mathrm{x}$ & $\mathrm{x}$ & $\mathrm{X}$ & $\mathbf{X}$ & $\mathrm{x}$ & $\mathrm{x}$ & $\mathrm{x}$ \\
\hline Mississippi River below Hickman, Ky. & LM 916.8 & $\mathrm{x}$ & $\mathrm{x}$ & $\mathrm{x}$ & $\mathrm{x}$ & $\mathrm{X}$ & $\mathrm{x}$ & \\
\hline Mississippi River at Fulton, Tenn. & LM 777.3 & & $\mathrm{X}$ & $\mathrm{x}$ & & $\mathrm{x}$ & & \\
\hline Mississippi River below Fulton, Tenn. & LM 773.5 & & & & $\mathrm{x}$ & & $\mathrm{x}$ & \\
\hline $\begin{array}{l}\text { Mississippi River above Hatchie River, } \\
\text { Tenn. }\end{array}$ & LM 773 & $\mathrm{X}$ & & & & & & \\
\hline Hatchie River at Mile 1.0, Miss. & - & $\mathrm{X}$ & & & & & & \\
\hline Mississippi River below Memphis, Tenn. & LM 731.2 & & & & & & & $\mathrm{X}$ \\
\hline Mississippi River at Helena, Ark. & LM 663.9 & $\mathrm{x}$ & $\mathrm{x}$ & $\mathrm{x}$ & $\mathrm{x}$ & $\mathrm{x}$ & $\mathrm{X}$ & \\
\hline White River at Mile 11.5, Ark. & WH 11.5 & $\mathrm{X}$ & $\mathrm{x}$ & $\mathrm{x}$ & $\mathrm{X}$ & $\mathrm{X}$ & & \\
\hline White River at Mile 1.0, Ark. & WH 1.0 & & & & & & $\mathrm{x}$ & $\mathrm{x}$ \\
\hline Arkansas River at Mile 55.9, Ark. & AR 55.9 & $\mathrm{X}$ & & & & & & \\
\hline Arkansas River at Pendleton, Ark. & AR 22.4 & & & & $\mathrm{x}$ & $\mathrm{x}$ & & \\
\hline Arkansas River at Mile 1.0, Ark. & AR 1.0 & & & $\mathrm{x}$ & & & $\mathrm{X}$ & $\mathrm{x}$ \\
\hline Mississippi River above Arkansas City, Ark. & LM 566.0 & $\mathbf{X}$ & $\mathbf{X}$ & $\mathrm{x}$ & $\mathrm{x}$ & $\mathrm{x}$ & $\mathrm{x}$ & \\
\hline Mississippi River below Arkansas City, Ark. & LM 551.7 & & & & & & & $\mathrm{X}$ \\
\hline Yazoo River at Mile 10, Miss. & YZ 10.0 & & $\mathrm{X}$ & $\mathrm{X}$ & & & & \\
\hline Yazoo River below Steele Bayou, Miss. & YZ 9.0 & & & & $\mathrm{x}$ & $\mathrm{X}$ & & $\mathrm{x}$ \\
\hline Yazoo River at Mile 1.0, Miss. & YZ 1.0 & & & & & & $\mathrm{x}$ & \\
\hline Mississippi River below Vicksburg, Miss. & LM 433.4 & $x$ & $\mathrm{X}$ & $\mathrm{x}$ & $\mathrm{X}$ & $\mathrm{X}$ & $\mathrm{x}$ & $\mathrm{X}$ \\
\hline $\begin{array}{l}\text { Old River Outflow Channel near Knox } \\
\text { Landing, La. }\end{array}$ & OR 5.5 & $\mathrm{X}$ & $x$ & $\mathrm{x}$ & $\mathrm{X}$ & $\mathrm{X}$ & & \\
\hline Mississippi River near St. Francisville, La. & LM 266.4 & $\mathrm{X}$ & $\mathrm{x}$ & $\mathrm{X}$ & $\mathrm{X}$ & $\mathrm{X}$ & $\mathrm{X}$ & $x$ \\
\hline Mississippi River at Plaquemine, La. & LM 208 & $\mathrm{X}$ & & & & & & \\
\hline Mississippi River at Union, La. & LM 167 & $x$ & & & & & & \\
\hline Mississippi River at Luling Bridge, La. & LM 122 & $X$ & & & & & & \\
\hline Mississippi River below Belle Chasse, La. & LM 73.1 & $\mathrm{X}$ & $\mathrm{X}$ & $\mathrm{X}$ & $\mathrm{X}$ & $\mathrm{x}$ & $\mathrm{X}$ & $X$ \\
\hline
\end{tabular}


Table 2.-Trace-element concentrations for field blanks taken during the July-August 1987 cruise on August 9, 1987

$[<$, less than the detection limit; $\mu \mathrm{g} / \mathrm{L}$, micrograms per liter]

\begin{tabular}{|c|c|c|c|c|c|}
\hline Trace element & $\begin{array}{c}\text { Tefion-lined tank } \\
(\mu g / L)\end{array}$ & 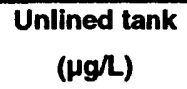 & Trace element & $\begin{array}{c}\text { Teflon-lined tank } \\
(\mu g / L)\end{array}$ & $\begin{array}{c}\text { Unlined tank } \\
\text { ( } \mu g / \text { ) }\end{array}$ \\
\hline Aluminum & 2.4 & 1.0 & Lead & 0.17 & 7.9 \\
\hline Arsenic & $<0.6$ & $<0.6$ & Lithium & $<0.03$ & $<0.03$ \\
\hline Barium & 7.6 & 0.12 & Manganese & 0.12 & 0.28 \\
\hline Beryllium & $<0.02$ & $<0.02$ & Molybdenum & $<0.09$ & $<0.09$ \\
\hline Boron & $<0.4$ & $<0.4$ & Nickel & 0.17 & 0.47 \\
\hline Cadmium & $<0.1$ & 0.15 & Strontium & 0.15 & 0.03 \\
\hline Chromium & 0.36 & 1.2 & Thallium & $<0.05$ & $<0.05$ \\
\hline Cobalt & 0.25 & 0.02 & Uranium & $<0.06$ & $<0.06$ \\
\hline Copper & 0.44 & 3.8 & Vanadium & $<0.07$ & $<0.07$ \\
\hline Iron & $<5$ & 13 & Zinc & 3.9 & 5.9 \\
\hline
\end{tabular}

Table 3.-Trace-element concentrations for field blanks taken during the November-December 1987 cruise

$[<$, less than the detection limit; --, not determined; $\mu \mathrm{g} / \mathrm{L}$, micrograms per liter]

\begin{tabular}{|c|c|c|c|c|c|c|c|}
\hline Trace element & $\begin{array}{l}\text { Date } \\
1987\end{array}$ & $\begin{array}{c}\text { Deionized } \\
\text { Water } \\
(\mu g /)\end{array}$ & $\begin{array}{c}\text { Teflon bag } \\
\text { sampler } \\
(\mu g /)\end{array}$ & $\begin{array}{l}\text { Sieve } \\
(\mu g / L)\end{array}$ & $\begin{array}{l}\text { Splitter } \\
((\mu g /))\end{array}$ & $\begin{array}{l}\text { Centrifuge } \\
\text { effluent } \\
\text { ( } \mu g / \text { L) }\end{array}$ & $\begin{array}{c}\text { Ultrafilter } \\
\text { permeate } \\
(\mu g /)\end{array}$ \\
\hline \multirow[t]{3}{*}{ Aluminum } & $12-6$ & 7.2 & - & - & - & - & - \\
\hline & $12-13$ & 17 & - & - & - & - & - \\
\hline & $12-19$ & $<0.2$ & 2.9 & 3.8 & 18 & 21 & 4.3 \\
\hline \multirow[t]{3}{*}{ Arsenic } & $12-6$ & $<0.6$ & - & - & - & - & - \\
\hline & $12-13$ & 2.7 & - & - & - & - & - \\
\hline & $12-19$ & $<0.6$ & $<0.6$ & $<0.6$ & $<0.6$ & $<0.6$ & $<0.6$ \\
\hline \multirow[t]{3}{*}{ Barium } & $12-6$ & 9.3 & - & -- & - & - & - \\
\hline & $12-13$ & 5.6 & - & - & - & - & - \\
\hline & $12-19$ & $<0.1$ & 0.64 & 0.65 & 1.3 & 3.3 & 2.9 \\
\hline \multirow[t]{3}{*}{ Beryllium } & $12-6$ & $<0.02$ & - & - & - & - & - \\
\hline & $12-13$ & $<0.02$ & - & - & - & - & - \\
\hline & $12-19$ & $<0.02$ & $<0.02$ & $<0.02$ & $<0.02$ & $<0.02$ & $<0.02$ \\
\hline \multirow[t]{3}{*}{ Boron } & $12-6$ & 2.9 & - & - & - & - & - \\
\hline & $12-13$ & 15 & - & - & - & - & - \\
\hline & $12-19$ & 9.4 & 8.4 & 8.9 & 8.9 & 9.2 & 9.8 \\
\hline \multirow[t]{3}{*}{ Cadmium } & $12-6$ & $<0.1$ & - & - & - & -- & - \\
\hline & $12-13$ & $<0.1$ & - & - & - & -- & - \\
\hline & $12-19$ & $<0.1$ & $<0.1$ & $<0.1$ & $<0.1$ & 0.29 & 0.21 \\
\hline
\end{tabular}


Table 3.-Trace-element concentrations for field blanks taken during the November-December 1987 cruise - Continued

\begin{tabular}{|c|c|c|c|c|c|c|c|}
\hline Trace element & $\begin{array}{l}\text { Date } \\
1987\end{array}$ & $\begin{array}{c}\text { Deionized } \\
\text { Water } \\
(\mu g / L)\end{array}$ & $\begin{array}{c}\text { Teflon bag } \\
\text { sampler } \\
\text { ( } \mu g / \text { L) }\end{array}$ & $\begin{array}{l}\text { Sieve } \\
(\mu g / L)\end{array}$ & $\begin{array}{l}\text { Splitter } \\
\text { ( } \mu g / \text { ) }\end{array}$ & $\begin{array}{c}\text { Centrifuge } \\
\text { effluent } \\
\text { ( } \mu g / L)\end{array}$ & $\begin{array}{c}\text { Ultrafliter } \\
\text { permeate } \\
(\mu g /)\end{array}$ \\
\hline \multirow[t]{3}{*}{ Chromium } & $12-6$ & 0.05 & - & - & - & - & - \\
\hline & $12-13$ & 1.3 & - & - & - & - & - \\
\hline & $12-19$ & $<0.2$ & $<0.2$ & $<0.2$ & $<0.2$ & 0.23 & 0.24 \\
\hline \multirow[t]{3}{*}{ Cobalt } & $12-6$ & 0.11 & - & - & - & - & - \\
\hline & $12-13$ & 0.1 & - & - & - & - & - \\
\hline & $12-19$ & $<0.008$ & $<0.008$ & $<0.008$ & 0.018 & 0.042 & 0.041 \\
\hline \multirow[t]{3}{*}{ Copper } & $12-6$ & 0.17 & - & - & - & - & $\cdots$ \\
\hline & $12-13$ & 0.35 & - & - & -- & - & - \\
\hline & $12-19$ & 0.21 & 0.13 & 0.15 & 0.24 & 3.5 & 1.8 \\
\hline \multirow[t]{3}{*}{ Iron } & $12-6$ & $<5$ & - & - & - & - & - \\
\hline & $12-13$ & $<5$ & - & - & - & - & - \\
\hline & $12-19$ & $<5$ & $<5$ & $<5$ & $<5$ & 16 & $<5$ \\
\hline \multirow[t]{3}{*}{ Lead } & $12-6$ & $<0.06$ & - & - & - & - & - \\
\hline & $12-13$ & $<0.06$ & -- & - & - & - & - \\
\hline & $12-19$ & $<0.06$ & $<0.06$ & $<0.06$ & $<0.06$ & 0.12 & $<0.06$ \\
\hline \multirow[t]{3}{*}{ Lithium } & $12-6$ & 0.87 & - & - & - & - & - \\
\hline & $12-13$ & 0.47 & - & - & - & - & - \\
\hline & $12-19$ & $<0.03$ & 0.26 & 0.28 & 0.55 & 0.66 & 0.66 \\
\hline \multirow[t]{3}{*}{ Manganese } & $12-6$ & 0.71 & - & - & - & - & - \\
\hline & 12.13 & 2.2 & - & - & - & - & $\cdots$ \\
\hline & $12-19$ & 0.14 & 0.14 & 0.17 & 0.42 & 0.89 & 0.69 \\
\hline \multirow[t]{3}{*}{ Molybdenum } & $12-6$ & 0.11 & - & - & - & - & - \\
\hline & $12-13$ & $<0.09$ & - & - & - & - & - \\
\hline & $12-19$ & $<0.09$ & $<0.09$ & $<0.09$ & $<0.09$ & 0.14 & 0.13 \\
\hline \multirow[t]{3}{*}{ Nickel } & $12-6$ & 2.5 & - & - & - & - & - \\
\hline & $12-13$ & 1.5 & -- & -- & - & -- & - \\
\hline & $12-19$ & $<0.03$ & 0.17 & 0.23 & 0.46 & 2.1 & 2.1 \\
\hline \multirow[t]{3}{*}{ Strontium } & $12-6$ & 18 & - & - & - & - & - \\
\hline & $12-13$ & 9 & - & - & - & - & - \\
\hline & $12-19$ & 0.03 & 1.3 & 1.3 & 2.6 & 5.9 & 6 \\
\hline \multirow[t]{3}{*}{ Thallium } & $12-6$ & $<.05$ & -- & - & - & - & - \\
\hline & $12-13$ & $<.05$ & - & - & - & - & - \\
\hline & $12-19$ & $<.05$ & $<.05$ & $<.05$ & $<.05$ & $<.05$ & $<.05$ \\
\hline
\end{tabular}


Table 3.--Trace-element concentrations for field blanks taken during the November-December 1987 cruise - Continued

\begin{tabular}{|c|c|c|c|c|c|c|c|}
\hline Trace element & $\begin{array}{l}\text { Date } \\
1987\end{array}$ & $\begin{array}{c}\text { Delonized } \\
\text { Water } \\
(\mu g /)\end{array}$ & $\begin{array}{c}\text { Tefion bag } \\
\text { sampler } \\
(\mu g \Omega)\end{array}$ & $\begin{array}{l}\text { Sieve } \\
(\mu g /)\end{array}$ & $\begin{array}{c}\text { Spiitter } \\
\text { ( } \mu g / \text { ) }\end{array}$ & $\begin{array}{c}\text { Centrifuge } \\
\text { effluent } \\
(\mu g /)\end{array}$ & $\begin{array}{c}\text { Ultrafilter } \\
\text { permeate } \\
(\mu g / L)\end{array}$ \\
\hline \multirow[t]{3}{*}{ Uranium } & $12-6$ & 0.07 & - & - & - & - & - \\
\hline & $12-13$ & $<0.06$ & - & - & - & - & - \\
\hline & $12-19$ & $<0.06$ & $<0.06$ & $<0.06$ & $<0.06$ & $<0.06$ & $<0.06$ \\
\hline \multirow[t]{3}{*}{ Vanadium } & $12-6$ & 0.28 & - & - & - & - & - \\
\hline & $12-13$ & 23 & - & - & - & - & - \\
\hline & $12-19$ & 1.4 & 0.48 & 0.29 & 0.29 & 0.35 & 0.3 \\
\hline \multirow[t]{3}{*}{ Zinc } & $12-6$ & 48 & - & - & -- & - & - \\
\hline & $12-13$ & 4.8 & - & - & - & - & - \\
\hline & $12-19$ & 2.3 & 2 & 1.9 & 4.2 & 4.8 & 3.8 \\
\hline
\end{tabular}

Table 4.--Trace-element concentrations for field blanks taken during the May-June 1988 cruise

$[<$, less than the detection limit; --, not determined; $\mu \mathrm{g} / \mathrm{L}$, micrograms per liter $]$

\begin{tabular}{|c|c|c|c|c|c|c|c|c|c|c|}
\hline $\begin{array}{l}\text { Trace } \\
\text { element }\end{array}$ & $\begin{array}{l}\text { Date } \\
1988\end{array}$ & $\begin{array}{c}\text { Deionlzed } \\
\text { Water } \\
(\mu g /))\end{array}$ & $\begin{array}{c}\text { Process } \\
\text { Blank' } \\
\text { ( } / \mathrm{g} / \mathrm{L})\end{array}$ & $\begin{array}{c}\text { Process } \\
\text { Blank } \\
(\mu g / \text { ) }\end{array}$ & $\begin{array}{c}\text { Splitter' } \\
\text { ( } \mu g / L)\end{array}$ & $\begin{array}{c}\text { Splitter } \\
\text { ( } \mu g / \text { ) }\end{array}$ & $\begin{array}{c}\text { Centrifuge } \\
\text { effluent' } \\
(\mu g /)\end{array}$ & $\begin{array}{c}\text { Centrifuge } \\
\text { effluent } \\
\text { ( } \mu g / \text { ) }\end{array}$ & $\begin{array}{c}\text { Ultrafilter } \\
\text { permeate' } \\
(\mu g /)\end{array}$ & $\begin{array}{c}\text { Ultrafilter } \\
\text { permeate } \\
\text { ( } \mu g / \text { ) }\end{array}$ \\
\hline \multirow[t]{4}{*}{ Aluminum } & $5-15$ & $<0.2$ & - & - & - & - & - & - & - & - \\
\hline & $5-20$ & $<0.2$ & - & - & - & - & - & -- & - & -- \\
\hline & 5.31 & $<0.2$ & 1.4 & 1.5 & 0.68 & 0.31 & 4.9 & 1.8 & 1.2 & $<0.2$ \\
\hline & 6.8 & $<0.2$ & -- & - & 2.4 & - & 9.8 & - & 1.3 & - \\
\hline \multirow[t]{4}{*}{ Arsenic } & $5-15$ & $<0.6$ & -- & -- & - & - & - & - & - & - \\
\hline & $5-20$ & $<0.6$ & - & - & - & - & - & - & - & - \\
\hline & $5-31$ & $<0.6$ & $<0.6$ & $<0.6$ & $<0.6$ & $<0.6$ & $<0.6$ & $<0.6$ & $<0.6$ & $<0.6$ \\
\hline & $6-8$ & $<0.6$ & - & - & $<0.6$ & - & $<0.6$ & - & $<0.6$ & - \\
\hline \multirow[t]{4}{*}{ Barium } & 5.15 & $<0.1$ & - & - & - & - & - & - & - & - \\
\hline & $5-20$ & $<0.1$ & -- & -- & - & - & - & - & - & - \\
\hline & $5-31$ & $<0.1$ & 0.13 & 0.14 & 0.11 & 0.11 & 0.88 & 0.66 & 0.52 & 0.32 \\
\hline & $6-8$ & $<0.1$ & -- & -- & $<0.1$ & - & 1.6 & -- & 0.52 & -- \\
\hline \multirow[t]{4}{*}{ Beryllium } & $5-15$ & $<0.02$ & - & - & - & - & - & - & - & - \\
\hline & $5-20$ & $<0.02$ & -- & -- & - & - & - & - & - & - \\
\hline & $5-31$ & $<0.02$ & $<0.02$ & $<0.02$ & $<0.02$ & $<0.02$ & $<0.02$ & $<0.02$ & $<0.02$ & $<0.02$ \\
\hline & $6-8$ & $<0.02$ & - & - & $<0.02$ & - & $<0.02$ & - & $<0.02$ & - \\
\hline \multirow[t]{4}{*}{ Boron } & $5-15$ & $<0.4$ & - & -- & - & - & -- & -- & -- & - \\
\hline & $5-20$ & $<0.4$ & - & - & - & - & - & - & -- & - \\
\hline & $5-31$ & $<0.4$ & $<0.4$ & $<0.4$ & $<0.4$ & $<0.4$ & $<0.4$ & $<0.4$ & 0.61 & $<0.4$ \\
\hline & $6-8$ & $<0.4$ & - & - & $<0.4$ & - & 0.73 & - & 0.51 & -- \\
\hline
\end{tabular}


Table 4.-Trace-element concentrations for field blanks taken during the May-June 1988 cruise Continued

\begin{tabular}{|c|c|c|c|c|c|c|c|c|c|c|}
\hline $\begin{array}{l}\text { Trace } \\
\text { element }\end{array}$ & $\begin{array}{l}\text { Date } \\
1988\end{array}$ & $\begin{array}{c}\text { Deionized } \\
\text { Water } \\
(\mu g /)\end{array}$ & $\begin{array}{c}\text { Process } \\
\text { Blank' } \\
\text { ( } \mu g / \text { ) }\end{array}$ & $\begin{array}{c}\text { Process } \\
\text { Blank } \\
\text { ( } \mu g / \text { ) }\end{array}$ & $\begin{array}{c}\text { Splitter' } \\
\text { ( } \mu g / \text { ) }\end{array}$ & $\begin{array}{c}\text { Splitter } \\
\left.\text { ( }{ }^{2} / \mathrm{L}\right)\end{array}$ & $\begin{array}{c}\text { Centrlfuge } \\
\text { effluent' } \\
(\mu g / L)\end{array}$ & $\begin{array}{c}\text { Centrlfuge } \\
\text { effluent } \\
\text { ( } \mu g / L)\end{array}$ & $\begin{array}{l}\text { Ultrafilter } \\
\text { permeate' } \\
(\mu g /) \text { ) }\end{array}$ & $\begin{array}{c}\text { Ultrafilter } \\
\text { permeate } \\
(\mu g /)\end{array}$ \\
\hline \multirow[t]{4}{*}{ Cadmium } & $5-15$ & $<0.1$ & - & - & - & - & -- & - & - & - \\
\hline & $5-20$ & $<0.1$ & - & - & - & - & - & - & - & -- \\
\hline & $5-31$ & $<0.1$ & 0.11 & $<0.1$ & $<0.1$ & $<0.1$ & 2 & 1.8 & 1.5 & 0.92 \\
\hline & $6-8$ & $<0.1$ & - & - & $<0.1$ & -- & 5.6 & - & 0.91 & - \\
\hline \multirow[t]{4}{*}{ Chromium } & $5-15$ & $<0.2$ & - & - & - & - & -- & - & - & - \\
\hline & $5-20$ & $<0.2$ & - & - & - & - & - & - & - & - \\
\hline & $5-31$ & $<0.2$ & $<0.2$ & $<0.2$ & $<0.2$ & $<0.2$ & $<0.2$ & $<0.2$ & $<0.2$ & $<0.2$ \\
\hline & $6-8$ & $<0.2$ & - & - & $<0.2$ & - & 0.26 & - & $<0.2$ & - \\
\hline \multirow[t]{4}{*}{ Cobalt } & $5-15$ & $<0.008$ & - & - & - & - & -- & - & - & - \\
\hline & $5-20$ & $<0.008$ & - & - & -- & -. & -- & - & - & - \\
\hline & $5-31$ & $<0.008$ & $<0.008$ & 0.009 & $<0.008$ & $<0.008$ & $<0.008$ & $<0.008$ & $<0.008$ & $<0.008$ \\
\hline & $6-8$ & $<0.008$ & - & - & $<0.008$ & - & 0.033 & - & 0.016 & - \\
\hline \multirow[t]{4}{*}{ Copper } & 5.15 & $<0.02$ & - & - & - & - & - & - & - & - \\
\hline & $5-20$ & 0.029 & - & - & - & - & - & - & - & - \\
\hline & 5.31 & $<0.02$ & 0.091 & 0.17 & 0.24 & 0.048 & 0.36 & 0.28 & 1 & 1.2 \\
\hline & $6-8$ & 0.14 & - & - & 0.1 & - & 2 & - & 0.47 & - \\
\hline \multirow[t]{4}{*}{ Iron } & $5-15$ & $<5$ & - & - & - & - & - & - & - & - \\
\hline & $5-20$ & $<5$ & - & - & - & - & - & - & - & - \\
\hline & $5-31$ & $<5$ & $<5$ & $<5$ & $<5$ & $<5$ & $<5$ & $<5$ & $<5$ & $<5$ \\
\hline & $6-8$ & $<5$ & - & - & $<5$ & - & 23 & - & $<5$ & - \\
\hline \multirow[t]{4}{*}{ Lead } & $5-15$ & $<0.06$ & - & - & - & - & - & - & - & - \\
\hline & $5-20$ & $<0.06$ & - & - & - & - & - & - & - & - \\
\hline & $5-31$ & $<0.06$ & $<0.06$ & $<0.06$ & $<0.06$ & $<0.06$ & 0.069 & $<0.06$ & 0.089 & $<0.06$ \\
\hline & $6-8$ & $<0.06$ & - & -- & $<0.06$ & - & 0.25 & - & 0.07 & - \\
\hline \multirow[t]{4}{*}{ Lithium } & $5-15$ & $<0.03$ & - & - & - & - & - & - & - & - \\
\hline & $5-20$ & $<0.03$ & - & - & - & - & - & - & - & - \\
\hline & $5-31$ & $<0.03$ & $<0.03$ & $<0.03$ & $<0.03$ & $<0.03$ & 0.049 & $<0.03$ & 0.12 & 0.032 \\
\hline & $6-8$ & $<0.03$ & - & -- & 0.22 & - & 0.36 & - & 0.28 & - \\
\hline \multirow[t]{4}{*}{ Manganese } & $5-15$ & $<0.06$ & - & - & - & - & - & - & - & - \\
\hline & $5-20$ & $<0.06$ & - & - & - & - & - & - & - & - \\
\hline & $5-31$ & $<0.06$ & 0.43 & 0.46 & 0.4 & 0.43 & 1.5 & 1.3 & 1.6 & 0.97 \\
\hline & $6-8$ & $<0.06$ & - & - & 0.21 & - & 1.2 & - & 0.78 & - \\
\hline \multirow[t]{4}{*}{ Molybdenum } & 5.15 & $<0.09$ & - & - & - & - & - & - & - & - \\
\hline & $5-20$ & $<0.09$ & - & - & - & - & - & - & - & - \\
\hline & 5.31 & $<0.09$ & $<0.09$ & $<0.09$ & $<0.09$ & $<0.09$ & $<0.09$ & $<0.09$ & $<0.09$ & $<0.09$ \\
\hline & $6-8$ & $<0.09$ & - & -. & $<0.09$ & - & $<0.09$ & - & $<0.09$ & - \\
\hline
\end{tabular}


Table 4.-Trace-element concentrations for field blanks taken during the May-June 1988 cruise Continued

\begin{tabular}{|c|c|c|c|c|c|c|c|c|c|c|}
\hline $\begin{array}{l}\text { Trace } \\
\text { element }\end{array}$ & $\begin{array}{l}\text { Date } \\
1988\end{array}$ & $\begin{array}{c}\text { Delonlzed } \\
\text { Water } \\
(\mu g / L)\end{array}$ & $\begin{array}{c}\text { Process } \\
\text { Blank' } \\
\text { ( } \mu g / L)\end{array}$ & $\begin{array}{c}\text { Process } \\
\text { Blank } \\
\text { ( } \mu g / L)\end{array}$ & $\begin{array}{c}\text { Splltter' } \\
\text { ( } \mu g / /)\end{array}$ & $\begin{array}{c}\text { Splitter } \\
\text { ( } \mu g / L)\end{array}$ & $\begin{array}{c}\text { Centrlfuge } \\
\text { effluent' } \\
(\mu g /)\end{array}$ & $\begin{array}{c}\text { Centrifuge } \\
\text { effluent }^{2} \\
(\mu g /)\end{array}$ & $\begin{array}{l}\text { Ultrafilter } \\
\text { permeate' } \\
(\mu g / L)\end{array}$ & $\begin{array}{c}\text { Ultrafilter } \\
\text { permeate } \\
(\mu g / L)\end{array}$ \\
\hline \multirow[t]{4}{*}{ Nickel } & 5.15 & $<0.03$ & - & -- & $\overline{-}$ & - & - & 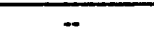 & - & - \\
\hline & $5-20$ & $<0.03$ & - & - & - & - & - & - & - & - \\
\hline & $5-31$ & $<0.03$ & 0.11 & 0.11 & 0.1 & 0.092 & 0.46 & 0.32 & 0.74 & 0.29 \\
\hline & $6-8$ & $<0.03$ & - & - & 0.09 & - & 0.75 & - & 0.37 & - \\
\hline \multirow[t]{4}{*}{ Strontium } & $5-15$ & $<0.01$ & - & - & - & - & - & - & - & - \\
\hline & $5-20$ & $<0.01$ & - & - & - & - & - & - & - & - \\
\hline & $5-31$ & $<0.01$ & 0.15 & 0.11 & 0.12 & 0.12 & 0.65 & 0.32 & 0.85 & 0.32 \\
\hline & $6-8$ & $<0.01$ & - & - & 0.064 & - & 2.7 & - & 1.2 & - \\
\hline \multirow[t]{4}{*}{ Thallium } & $5-15$ & $<0.05$ & - & - & - & - & - & -- & - & - \\
\hline & $5-20$ & $<0.05$ & - & - & - & - & - & - & - & -- \\
\hline & $5-31$ & $<0.05$ & $<0.05$ & $<0.05$ & $<0.05$ & $<0.05$ & $<0.05$ & $<0.05$ & $<0.05$ & $<0.05$ \\
\hline & 6.8 & $<0.05$ & -. & - & $<0.05$ & - & $<0.05$ & -. & $<0.05$ & -. \\
\hline \multirow[t]{4}{*}{ Uranium } & 5.15 & $<0.06$ & - & - & - & - & - & - & - & - \\
\hline & $5-20$ & $<0.06$ & - & - & - & - & - & -- & - & - \\
\hline & $5-31$ & $<0.06$ & $<0.06$ & $<0.06$ & $<0.06$ & $<0.06$ & $<0.06$ & $<0.06$ & $<0.06$ & $<0.06$ \\
\hline & $6-8$ & $<0.06$ & - & - & $<0.06$ & - & $<0.06$ & - & $<0.06$ & - \\
\hline \multirow[t]{4}{*}{ Vanadium } & $5-15$ & $<0.07$ & - & - & - & - & - & - & - & - \\
\hline & $5-20$ & $<0.07$ & - & - & - & - & - & - & - & - \\
\hline & $5-31$ & $<0.07$ & $<0.07$ & $<0.07$ & $<0.07$ & $<0.07$ & $<0.07$ & $<0.07$ & $<0.07$ & $<0.07$ \\
\hline & $6-8$ & $<0.07$ & - & - & $<0.07$ & - & 0.21 & -- & $<0.07$ & - \\
\hline \multirow[t]{4}{*}{ Zinc } & $5-15$ & $<0.08$ & - & - & - & - & -- & - & - & - \\
\hline & $5-20$ & 0.5 & - & - & - & - & -- & -. & - & - \\
\hline & $5-31$ & $<0.08$ & 0.53 & 2 & 0.51 & 0.59 & 2.5 & 2.6 & 4.4 & 2.3 \\
\hline & $6-8$ & 0.46 & - & - & 0.89 & - & 1.8 & - & 2.7 & - \\
\hline
\end{tabular}

${ }^{1}$ Processed using unstained Teflon-coated chum splitters.

${ }^{2}$ Processed using rust stained Teflon-coated chum splitters. 
Table 5.-- Trace-element concentrations for field blanks taken during the May-June 1988 cruise on May 18, 1988

$[<$, less than the detection limit; $\mu \mathrm{g} / \mathrm{L}$, micrograms per liter]

\begin{tabular}{|c|c|c|c|c|c|c|c|c|c|c|}
\hline $\begin{array}{l}\text { Trace } \\
\text { element }\end{array}$ & $\begin{array}{c}\text { Teflon bag } \\
\text { A } \\
(\mu g / L)\end{array}$ & $\begin{array}{c}\text { Teflon bag } \\
\text { B } \\
(\mu g / L)\end{array}$ & $\begin{array}{c}\text { Splitter } \\
\text { A } \\
\text { ( } \mu g / \text { ) }\end{array}$ & $\begin{array}{c}\text { Splitter } \\
\text { B } \\
\text { ( } \mu g / L)\end{array}$ & $\begin{array}{c}\text { Sieve } \\
\text { A } \\
(\mu g /)\end{array}$ & $\begin{array}{c}\text { Sleve } \\
\text { B } \\
(\mu g /)\end{array}$ & $\begin{array}{c}\text { Splitter } \\
\text { composite } \\
(\mu g / L)\end{array}$ & $\begin{array}{l}\text { Centrifuge } \\
\text { effluent } \\
\text { ( } \mu g / L)\end{array}$ & $\begin{array}{c}\text { Ultrafilter } \\
\text { retentate } \\
(\mu g / L)\end{array}$ & $\begin{array}{c}\text { Ultrafilter } \\
\text { permeate } \\
(\mu g / L)\end{array}$ \\
\hline Aluminum & $<0.2$ & $<0.2$ & 1.7 & $<0.2$ & $<0.2$ & $<0.2$ & 3.4 & 6.3 & 1.8 & $<0.2$ \\
\hline Arsenic & $<0.6$ & $<0.6$ & $<0.6$ & $<0.6$ & $<0.6$ & $<0.6$ & $<0.6$ & $<0.6$ & $<0.6$ & $<0.6$ \\
\hline Barium & $<0.1$ & $<0.1$ & $<0.1$ & $<0.1$ & $<0.1$ & $<0.1$ & 0.14 & 1 & 0.75 & 0.74 \\
\hline Beryllium & $<0.02$ & $<0.02$ & $<0.02$ & $<0.02$ & $<0.02$ & $<0.02$ & $<0.02$ & $<0.02$ & $<0.02$ & $<0.02$ \\
\hline Boron & $<0.4$ & $<0.4$ & $<0.4$ & $<0.4$ & $<0.4$ & $<0.4$ & $<0.4$ & 5.5 & $<0.4$ & $<0.4$ \\
\hline Cadmium & $<0.1$ & $<0.1$ & $<0.1$ & $<0.1$ & $<0.1$ & $<0.1$ & $<0.1$ & 2 & 3.1 & 1.2 \\
\hline Chromium & $<0.2$ & 0.22 & $<0.2$ & $<0.2$ & $<0.2$ & $<0.2$ & $<0.2$ & 0.75 & 0.35 & $<0.2$ \\
\hline Cobalt & $<0.008$ & $<0.008$ & $<0.008$ & $<0.008$ & $<0.008$ & $<0.008$ & $<0.008$ & 0.018 & 0.024 & $<0.008$ \\
\hline Copper & $<0.02$ & $<0.02$ & 0.026 & 0.027 & $<0.02$ & $<0.02$ & 0.062 & 21 & 0.6 & 0.26 \\
\hline Iron & $<5$ & $<5$ & $<5$ & $<5$ & $<5$ & $<5$ & $<5$ & 27 & 16 & $<5$ \\
\hline Lead & $<0.06$ & $<0.06$ & $<0.06$ & $<0.06$ & $<0.06$ & $<0.06$ & $<0.06$ & 0.13 & 0.13 & $<0.06$ \\
\hline Lithium & $<0.03$ & $<0.03$ & $<0.03$ & $<0.03$ & $<0.03$ & $<0.03$ & $<0.03$ & 0.29 & 0.1 & 0.09 \\
\hline Manganese & $<0.06$ & $<0.06$ & 0.39 & 0.12 & $<0.06$ & $<0.06$ & 0.61 & 12 & 4.9 & 4.7 \\
\hline Molybdenum & $<0.09$ & $<0.09$ & $<0.09$ & $<0.09$ & $<0.09$ & $<0.09$ & $<0.09$ & $<0.09$ & $<0.09$ & $<0.09$ \\
\hline Nickel & 0.046 & 0.033 & 0.064 & 0.085 & $<0.03$ & 0.057 & 0.086 & 0.9 & 1.4 & 0.66 \\
\hline Strontium & $<0.01$ & $<0.01$ & 0.049 & 0.032 & $<0.01$ & 0.019 & 0.09 & 1.3 & 1.1 & 1.1 \\
\hline Thallium & $<0.05$ & $<0.05$ & $<0.05$ & $<0.05$ & $<0.05$ & $<0.05$ & $<0.05$ & $<0.05$ & $<0.05$ & $<0.05$ \\
\hline Uranium & $<0.06$ & $<0.06$ & $<0.06$ & $<0.06$ & $<0.06$ & $<0.06$ & $<0.06$ & $<0.06$ & $<0.06$ & $<0.06$ \\
\hline Vanadium & $<0.07$ & $<0.07$ & $<0.07$ & $<0.07$ & $<0.07$ & $<0.07$ & $<0.07$ & 0.08 & $<0.07$ & $<0.07$ \\
\hline Zinc & $<0.08$ & 0.24 & 0.36 & 0.15 & $<0.08$ & 0.25 & 0.29 & 4.7 & 1.6 & 1.3 \\
\hline
\end{tabular}

Table 6.- Trace-element concentrations for field blanks taken during the March-April 1989 cruise

$[<$, less than the detection limit; --, not determined; $\mu \mathrm{g} / \mathrm{L}$, micrograms per liter $]$

\begin{tabular}{|c|c|c|c|c|c|c|c|}
\hline $\begin{array}{l}\text { Trace } \\
\text { eiement }\end{array}$ & $\begin{array}{l}\text { Date } \\
1989\end{array}$ & $\begin{array}{c}\text { Delonized } \\
\text { Water } \\
(\mu g / L)\end{array}$ & $\begin{array}{c}\text { Teflon bag } \\
\text { sampler } \\
(\mu g / L)\end{array}$ & $\begin{array}{l}\text { Sleve } \\
\text { ( } \mu g / \text { ) }\end{array}$ & $\begin{array}{c}\text { Splitter } \\
(\mu g / L)\end{array}$ & $\begin{array}{l}\text { Centrifuge } \\
\text { effiuent } \\
\text { ( } \mathrm{gg} / \mathrm{L})\end{array}$ & $\begin{array}{c}\text { Ultrafilter } \\
\text { permeate } \\
(\mu g / L)\end{array}$ \\
\hline \multirow[t]{3}{*}{ Aluminum } & $3-11$ & 0.47 & $<0.2$ & $<0.2$ & 1.1 & $<0.2$ & $<0.2$ \\
\hline & $3-25$ & $<0.2$ & - & 9.9 & 10 & 8.1 & 3.5 \\
\hline & $3-31$ & $<0.2$ & - & $<0.2$ & 3.1 & $<0.2$ & $<0.2$ \\
\hline \multirow[t]{3}{*}{ Arsenic } & $3-11$ & $<0.6$ & $<0.6$ & $<0.6$ & $<0.6$ & $<0.6$ & $<0.6$ \\
\hline & $3-25$ & $<0.6$ & - & $<0.6$ & $<0.6$ & $<0.6$ & $<0.6$ \\
\hline & $3-31$ & $<0.6$ & - & $<0.6$ & $<0.6$ & $<0.6$ & $<0.6$ \\
\hline \multirow[t]{3}{*}{ Barium } & $3-11$ & 0.23 & 0.84 & 0.94 & 0.97 & 1.7 & 1.2 \\
\hline & $3-25$ & $<0.1$ & -- & $<0.1$ & $<0.1$ & $<0.1$ & $<0.1$ \\
\hline & $3-31$ & $<0.1$ & - & $<0.1$ & $<0.1$ & $<0.1$ & 0.18 \\
\hline \multirow[t]{3}{*}{ Beryllium } & $3-11$ & $<0.02$ & $<0.02$ & $<0.02$ & $<0.02$ & $<0.02$ & $<0.02$ \\
\hline & $3-25$ & $<0.02$ & - & $<0.02$ & $<0.02$ & $<0.02$ & $<0.02$ \\
\hline & $3-31$ & $<0.02$ & - & $<0.02$ & $<0.02$ & $<0.02$ & $<0.02$ \\
\hline
\end{tabular}


Table 6.- Trace-element concentrations for field blanks taken during the March-April 1989 cruise - Continued

\begin{tabular}{|c|c|c|c|c|c|c|c|}
\hline $\begin{array}{l}\text { Trace } \\
\text { element }\end{array}$ & $\begin{array}{l}\text { Date } \\
1989 \\
\end{array}$ & $\begin{array}{c}\text { Delonlzed } \\
\text { Water } \\
(\mu g / L)\end{array}$ & $\begin{array}{c}\text { Teflon bag } \\
\text { sampler } \\
(\mu g / L)\end{array}$ & $\begin{array}{l}\text { Sleve } \\
\text { ( } \mu g / L)\end{array}$ & $\begin{array}{l}\text { Splltter } \\
\text { ( } \mu g / \text { L) }\end{array}$ & $\begin{array}{c}\text { Centrlfuge } \\
\text { effluent } \\
\text { ( } \mu g / \text { L) }\end{array}$ & $\begin{array}{c}\text { Ultrafilter } \\
\text { permeate } \\
(\mu g / L)\end{array}$ \\
\hline \multirow[t]{3}{*}{ Boron } & $3-11$ & $<0.4$ & $<0.4$ & $<0.4$ & $<0.4$ & $<0.4$ & $<0.4$ \\
\hline & $3-25$ & $<0.4$ & - & 29 & 28 & 28 & 30 \\
\hline & $3-31$ & $<0.4$ & - & $<0.4$ & 0.88 & 0.62 & 0.65 \\
\hline \multirow[t]{3}{*}{ Cadmium } & $3-11$ & $<0.1$ & $<0.1$ & $<0.1$ & $<0.1$ & 0.6 & $<0.1$ \\
\hline & $3-25$ & $<0.1$ & - & $<0.1$ & $<0.1$ & $<0.1$ & $<0.1$ \\
\hline & $3-31$ & $<0.1$ & - & $<0.1$ & $<0.1$ & $<0.1$ & $<0.1$ \\
\hline \multirow[t]{3}{*}{ Chromium } & $3-11$ & $<0.2$ & $<0.2$ & $<0.2$ & $<0.2$ & $<0.2$ & $<0.2$ \\
\hline & $3-25$ & $<0.2$ & - & $<0.2$ & $<0.2$ & 0.26 & $<0.2$ \\
\hline & $3-31$ & $<0.2$ & - & $<0.2$ & $<0.2$ & $<0.2$ & $<0.2$ \\
\hline \multirow[t]{3}{*}{ Cobalt } & 3.11 & $<0.008$ & 0.014 & 0.013 & 0.019 & 0.016 & 0.013 \\
\hline & $3-25$ & $<0.008$ & - & $<0.008$ & 0.009 & 0.016 & 0.012 \\
\hline & $3-31$ & 0.011 & - & 0.014 & 0.016 & $<0.008$ & $<0.008$ \\
\hline \multirow[t]{3}{*}{ Copper } & $3-11$ & $<0.02$ & $<0.02$ & $<0.02$ & $<0.02$ & 0.077 & $<0.02$ \\
\hline & $3-25$ & $<0.02$ & - & 0.92 & 0.82 & 0.7 & 0.27 \\
\hline & $3-31$ & $<0.02$ & - & 0.097 & 0.32 & 0.041 & 0.031 \\
\hline \multirow[t]{3}{*}{ Iron } & $3-11$ & $<5$ & $<5$ & $<5$ & $<5$ & $<5$ & $<5$ \\
\hline & $3-25$ & $<5$ & $<5$ & $<5$ & $<5$ & $<5$ & $<5$ \\
\hline & $3-31$ & $<5$ & $<5$ & $<5$ & $<5$ & $<5$ & $<5$ \\
\hline \multirow[t]{3}{*}{ Lead } & $3-11$ & $<0.06$ & $<0.06$ & $<0.06$ & $<0.06$ & 0.86 & $<0.06$ \\
\hline & $3-25$ & $<0.06$ & - & 0.14 & 0.14 & 0.13 & $<0.06$ \\
\hline & $3-31$ & $<0.06$ & - & $<0.06$ & $<0.06$ & $<0.06$ & $<0.06$ \\
\hline \multirow[t]{3}{*}{ Lithium } & $3-11$ & 0.16 & 0.37 & 0.34 & 0.33 & 0.3 & 0.36 \\
\hline & $3-25$ & $<0.03$ & - & 0.46 & 0.48 & 0.53 & 0.53 \\
\hline & $3-31$ & $<0.03$ & - & 0.46 & 0.62 & 0.6 & 0.58 \\
\hline \multirow[t]{3}{*}{ Manganese } & $3-11$ & 0.24 & 0.38 & 0.28 & 0.5 & 0.6 & 0.72 \\
\hline & $3-25$ & $<0.06$ & - & 0.11 & 0.18 & 0.28 & 0.37 \\
\hline & $3-31$ & $<0.06$ & - & 0.1 & 0.28 & 0.23 & 0.22 \\
\hline \multirow[t]{3}{*}{ Molybdenum } & $3-11$ & 0.27 & $<0.09$ & $<0.09$ & $<0.09$ & $<0.09$ & $<0.09$ \\
\hline & $3-25$ & $<0.09$ & - & $<0.09$ & $<0.09$ & $<0.09$ & $<0.09$ \\
\hline & 3-31 & $<0.09$ & - & $<0.09$ & $<0.09$ & $<0.09$ & $<0.09$ \\
\hline \multirow[t]{3}{*}{ Nickel } & $3-11$ & $<0.03$ & $<0.03$ & $<0.03$ & 0.12 & 0.2 & 1.3 \\
\hline & $3-25$ & $<0.03$ & - & 0.016 & $<0.03$ & 0.28 & 0.31 \\
\hline & $3-31$ & $<0.03$ & - & $<0.03$ & 0.12 & 0.061 & 0.24 \\
\hline \multirow[t]{3}{*}{ Strontium } & $3-11$ & 0.068 & 3.3 & 3.1 & 3 & 3 & 3.3 \\
\hline & $3-25$ & $<0.01$ & - & $<0.01$ & 0.053 & 0.14 & 0.69 \\
\hline & $3-31$ & $<0.01$ & - & 1.6 & 2 & 2 & 2.1 \\
\hline
\end{tabular}


Table 6.- Trace-element concentrations for field blanks taken during the March-April 1989 cruise - Continued

\begin{tabular}{|c|c|c|c|c|c|c|c|}
\hline $\begin{array}{l}\text { Trace } \\
\text { element }\end{array}$ & $\begin{array}{l}\text { Date } \\
1989\end{array}$ & $\begin{array}{c}\text { Deionlzed } \\
\text { Water } \\
\text { (pg/L) }\end{array}$ & $\begin{array}{c}\text { Tefion bag } \\
\text { sampler } \\
(\mu g / L)\end{array}$ & $\begin{array}{l}\text { Sleve } \\
\text { ( } \mu g / L)\end{array}$ & $\begin{array}{c}\text { Splltter } \\
\text { ( } \mu g / \text { ) }\end{array}$ & $\begin{array}{c}\text { Centrlfuge } \\
\text { effluent } \\
\text { ( } \mathrm{g} / \mathrm{L} \text { ) }\end{array}$ & $\begin{array}{c}\text { Ultrafilter } \\
\text { permeate } \\
\text { ( } \mu g / \text { L) }\end{array}$ \\
\hline \multirow[t]{3}{*}{ Thallium } & $3-11$ & $<.05$ & $<.05$ & $<.05$ & $<.05$ & $<.05$ & $<.05$ \\
\hline & $3-25$ & $<0.05$ & - & $<0.05$ & $<0.05$ & $<0.05$ & $<0.05$ \\
\hline & $3-31$ & $<0.05$ & - & $<0.05$ & $<0.05$ & $<0.05$ & $<0.05$ \\
\hline \multirow[t]{3}{*}{ Uranium } & $3-11$ & $<0.06$ & $<0.06$ & $<0.06$ & $<0.06$ & $<0.06$ & $<0.06$ \\
\hline & $3-25$ & $<0.06$ & - & $<0.06$ & $<0.06$ & $<0.06$ & $<0.06$ \\
\hline & $3-31$ & $<0.06$ & - & $<0.06$ & $<0.06$ & $<0.06$ & $<0.06$ \\
\hline \multirow[t]{3}{*}{ Vanadium } & $3-11$ & $<0.07$ & $<0.07$ & $<0.07$ & $<0.07$ & $<0.07$ & $<0.07$ \\
\hline & $3-25$ & $<0.07$ & - & $<0.07$ & $<0.07$ & 0.072 & $<0.07$ \\
\hline & $3-31$ & $<0.07$ & - & $<0.07$ & $<0.07$ & $<0.07$ & $<0.07$ \\
\hline \multirow[t]{3}{*}{ Zinc } & $3-11$ & 0.9 & 1.5 & 1.7 & 2.1 & 3.1 & 7 \\
\hline & 3.25 & 0.82 & - & 1.4 & 0.76 & 3.2 & 2.5 \\
\hline & $3-31$ & 0.94 & - & 1.1 & 1.2 & 4.2 & 1.3 \\
\hline
\end{tabular}

Table 7.- Trace-element concentrations for field blanks taken during the June 1989 cruise

$[<$, less than the detection limit; $\mu \mathrm{g} / \mathrm{L}$, micrograms per liter ]

\begin{tabular}{|c|c|c|c|c|c|c|}
\hline $\begin{array}{l}\text { Trace } \\
\text { element }\end{array}$ & $\begin{array}{l}\text { Date } \\
1989\end{array}$ & $\begin{array}{c}\text { Delonized } \\
\text { Water } \\
\text { ( } \mu g / \text { ) }\end{array}$ & $\begin{array}{l}\text { Sleve } \\
(\mu g /)\end{array}$ & $\begin{array}{l}\text { Splltter } \\
\text { ( } \mu g / \text { ) }\end{array}$ & $\begin{array}{l}\text { Centrifuge } \\
\text { effiuent } \\
\text { (pg/L) }\end{array}$ & $\begin{array}{c}\text { Uitrafilter } \\
\text { permeate } \\
(\mu g / L)\end{array}$ \\
\hline \multirow[t]{2}{*}{ Aluminum } & 6-06 & $<0.2$ & 3.6 & 3.6 & 3.2 & 20 \\
\hline & $6-21$ & 14 & 14 & 23 & 24 & 8.5 \\
\hline \multirow[t]{2}{*}{ Arsenic } & $6-06$ & $<0.6$ & $<0.6$ & 0.66 & $<0.6$ & $<0.6$ \\
\hline & $6-21$ & $<0.6$ & $<0.6$ & $<0.6$ & 0.68 & $<0.6$ \\
\hline \multirow[t]{2}{*}{ Barium } & $6-06$ & $<0.1$ & 2.8 & 2 & 2.4 & 2.8 \\
\hline & $6-21$ & 1.7 & 1.2 & 1.8 & 1.8 & 1.6 \\
\hline \multirow[t]{2}{*}{ Beryllium } & $6-06$ & 0.031 & 0.023 & $<0.02$ & $<0.02$ & 0.04 \\
\hline & $6-21$ & $<0.02$ & $<0.02$ & $<0.02$ & 0.032 & 0.02 \\
\hline \multirow[t]{2}{*}{ Boron } & $6-06$ & $<0.4$ & 2.5 & 1.8 & 2.9 & 2.2 \\
\hline & $6-21$ & 11 & 7.2 & 9.1 & 8.7 & 8.8 \\
\hline \multirow[t]{2}{*}{ Cadmium } & $6-06$ & $<0.1$ & $<0.1$ & $<0.1$ & 0.2 & 0.18 \\
\hline & $6-21$ & $<0.1$ & $<0.1$ & $<0.1$ & 3.8 & 0.12 \\
\hline \multirow[t]{2}{*}{ Chromium } & $6-06$ & $<0.2$ & $<0.2$ & $<0.2$ & $<0.2$ & $<0.2$ \\
\hline & $6-21$ & $<0.2$ & $<0.2$ & $<0.2$ & $<0.2$ & $<0.2$ \\
\hline \multirow[t]{2}{*}{ Cobalt } & $6-06$ & $<0.008$ & 0.041 & 0.036 & 0.044 & 0.046 \\
\hline & $6-21$ & 0.014 & $<0.008$ & $<0.008$ & 0.014 & $<0.008$ \\
\hline
\end{tabular}


Table 7. - Trace-element concentrations for field blanks taken during the June 1989 cruise Continued

\begin{tabular}{|c|c|c|c|c|c|c|}
\hline $\begin{array}{l}\text { Trace } \\
\text { element }\end{array}$ & $\begin{array}{l}\text { Date } \\
1989\end{array}$ & $\begin{array}{c}\text { Delonlzed } \\
\text { Water } \\
(\mu g /))\end{array}$ & $\begin{array}{l}\text { Sleve } \\
(\mu g / L)\end{array}$ & $\begin{array}{l}\text { Splltter } \\
\text { ( } \mu g / \text { ) }\end{array}$ & $\begin{array}{c}\text { Centrlfuge } \\
\text { effluent } \\
\text { ( } \mu g / L)\end{array}$ & $\begin{array}{c}\text { Ultrafilter } \\
\text { permeate } \\
\text { ( } \mu g / L)\end{array}$ \\
\hline \multirow[t]{2}{*}{ Copper } & 6-06 & $<0.02$ & $<0.02$ & 0.052 & 0.17 & 0.15 \\
\hline & $6-21$ & 0.57 & 0.28 & 0.3 & 0.47 & 0.037 \\
\hline \multirow[t]{2}{*}{ Iron } & $6-06$ & $<5$ & $<5$ & $<5$ & $<5$ & $<5$ \\
\hline & $6-21$ & $<5$ & $<5$ & 6 & 15 & $<5$ \\
\hline \multirow[t]{2}{*}{ Lead } & $6-06$ & $<0.06$ & 0.079 & $<0.06$ & $<0.06$ & $<0.06$ \\
\hline & $6-21$ & 0.14 & 0.084 & 0.31 & 0.28 & 0.068 \\
\hline \multirow[t]{2}{*}{ Lithium } & $6-06$ & $<0.03$ & 0.3 & 0.28 & 0.29 & 0.31 \\
\hline & $6-21$ & 0.48 & 0.27 & 0.4 & 0.39 & 0.38 \\
\hline \multirow[t]{2}{*}{ Manganese } & $6-06$ & $<0.06$ & 1.5 & 1.4 & 2.1 & 2.2 \\
\hline & $6-21$ & 0.57 & 0.49 & 0.7 & 0.88 & 0.49 \\
\hline \multirow[t]{2}{*}{ Molybdenum } & $6-06$ & $<0.09$ & $<0.09$ & $<0.09$ & $<0.09$ & $<0.09$ \\
\hline & $6-21$ & $<0.09$ & $<0.09$ & $<0.09$ & $<0.09$ & $<0.09$ \\
\hline \multirow[t]{2}{*}{ Nickel } & $6-06$ & $<0.03$ & 0.56 & 0.6 & 0.8 & 0.69 \\
\hline & $6-21$ & 0.17 & 0.096 & 0.25 & 0.52 & 0.32 \\
\hline \multirow[t]{2}{*}{ Strontium } & $6-06$ & 0.086 & 17 & 13 & 15 & 15 \\
\hline & $6-21$ & 5.2 & 3.2 & 4.2 & 4.5 & 4.2 \\
\hline \multirow[t]{2}{*}{ Thallium } & $6-06$ & $<0.05$ & $<0.05$ & $<0.05$ & $<0.05$ & $<0.05$ \\
\hline & $6-21$ & 0.12 & 0.19 & 0.074 & 0.22 & 0.13 \\
\hline \multirow[t]{2}{*}{ Uranium } & $6-06$ & $<0.06$ & $<0.06$ & $<0.06$ & $<0.06$ & $<0.06$ \\
\hline & $6-21$ & 0.2 & 0.52 & 0.12 & 0.46 & 0.17 \\
\hline \multirow[t]{2}{*}{ Vanadium } & $6-06$ & 0.074 & 0.09 & 0.071 & $<0.07$ & 0.075 \\
\hline & $6-21$ & $<0.07$ & $<0.07$ & $<0.07$ & 0.12 & 0.093 \\
\hline \multirow[t]{2}{*}{ Zinc } & $6-06$ & 2 & 1.2 & 1.2 & 2.9 & 2.2 \\
\hline & $6-21$ & 4.1 & 1.9 & 1.2 & 1.5 & 0.85 \\
\hline
\end{tabular}


Table 8.- Trace-element concentrations for field blanks taken during the February-March 1990 cruise on March 13, 1990

$[<$, less than the detection limit; $\mu \mathrm{g} / \mathrm{L}$, micrograms per liter ]

\begin{tabular}{lcccccc}
\hline $\begin{array}{l}\text { Trace } \\
\text { element }\end{array}$ & $\begin{array}{c}\text { Deionized } \\
\text { Water } \\
(\mu g /)\end{array}$ & $\begin{array}{c}\text { Nuclepore } \\
(\mu g /)\end{array}$ & $\begin{array}{c}\text { Sieve } \\
(\mu g /)\end{array}$ & $\begin{array}{c}\text { Splitter } \\
(\mu g / L)\end{array}$ & $\begin{array}{c}\text { Centrifuge } \\
\text { effluent } \\
(\mu g /)\end{array}$ & $\begin{array}{c}\text { Ultrafilter } \\
\text { permeate } \\
(\mu g / L)\end{array}$ \\
\hline Aluminum & 2.6 & 4.6 & 3.1 & 16 & 6.1 & 0.75 \\
Arsenic & 0.7 & 0.7 & 0.68 & 0.69 & 0.66 & $<0.6$ \\
Barium & $<0.1$ & $<0.1$ & $<0.1$ & $<0.1$ & $<0.1$ & $<0.1$ \\
Beryllium & $<0.02$ & $<0.02$ & $<0.02$ & $<0.02$ & $<0.02$ & $<0.02$ \\
Boron & 8.7 & 13 & 7 & 1.8 & 1.5 & 2.3 \\
Cadmium & $<0.1$ & $<0.1$ & $<0.1$ & $<0.1$ & 0.66 & $<0.1$ \\
Chromium & $<0.2$ & $<0.2$ & $<0.2$ & $<0.2$ & $<0.2$ & $<0.2$ \\
Cobait & $<0.008$ & $<0.008$ & $<0.008$ & $<0.008$ & $<0.008$ & $<0.008$ \\
Copper & 0.56 & 0.94 & 0.49 & 0.31 & 6.2 & 0.31 \\
Iron & 5 & 5 & $<5$ & 13 & 12 & $<5$ \\
Lead & $<0.06$ & $<0.06$ & $<0.06$ & $<0.06$ & $<0.06$ & $<0.06$ \\
Lithium & 1.1 & 1.5 & 1.1 & 0.66 & 0.65 & 0.65 \\
Manganese & $<0.06$ & 0.061 & $<0.06$ & 0.23 & 0.28 & 0.14 \\
Molybdenum & $<0.09$ & $<0.09$ & $<0.09$ & $<0.09$ & $<0.09$ & $<0.09$ \\
Nickel & $<0.02$ & $<0.02$ & $<0.02$ & $<0.02$ & $<0.02$ & $<0.02$ \\
Strontium & 0.63 & $<0.01$ & $<0.01$ & 0.49 & 0.29 & 1.2 \\
Thallium & $<0.05$ & $<0.05$ & $<0.05$ & $<0.05$ & $<0.05$ & $<0.05$ \\
Uranium & $<0.06$ & $<0.06$ & $<0.06$ & $<0.06$ & $<0.06$ & $<0.06$ \\
Vanadium & $<0.07$ & $<0.07$ & $<0.07$ & $<0.07$ & $<0.07$ & $<0.07$ \\
Zinc & 0.58 & 2.6 & 0.3 & 0.35 & 0.13 & 0.27 \\
\hline & & & & &
\end{tabular}

Table 9.-- Trace-element concentrations for field blanks taken during the May-June 1990 cruise on June 26, 1990

$[<$, less than the detection limit; $\mu \mathrm{g} / \mathrm{L}$, micrograms per liter ]

\begin{tabular}{lcccccc}
\hline $\begin{array}{l}\text { Trace } \\
\text { element }\end{array}$ & $\begin{array}{c}\text { Deionized } \\
\text { Water } \\
(\mu g / L)\end{array}$ & $\begin{array}{c}\text { Nuclepore } \\
(\mu g / L)\end{array}$ & $\begin{array}{c}\text { Sieve } \\
(\mu g /)\end{array}$ & $\begin{array}{c}\text { Splitter } \\
(\mu g / L)\end{array}$ & $\begin{array}{c}\text { Centrifuge } \\
\text { effluent } \\
(\mu g /)\end{array}$ & $\begin{array}{c}\text { Ultrafilter } \\
\text { permeate } \\
(\mu g / L)\end{array}$ \\
\hline Aluminum & 12 & 1.8 & 17 & 24 & 15 & 4.9 \\
Arsenic & $<0.6$ & $<0.6$ & $<0.6$ & $<0.6$ & $<0.6$ & $<0.6$ \\
Barium & 0.1 & $<0.1$ & 0.19 & 0.39 & 0.74 & 0.69 \\
Beryllium & $<0.02$ & $<0.02$ & $<0.02$ & 0.021 & 0.028 & 0.024 \\
Boron & 7.1 & 0.93 & 10 & 9.4 & 8.9 & 10 \\
Cadmium & $<0.1$ & $<0.1$ & $<0.1$ & $<0.1$ & 1.4 & 0.2 \\
Chromium & $<0.2$ & $<0.2$ & $<0.2$ & $<0.2$ & $<0.2$ & $<0.2$ \\
Cobalt & 0.0085 & $<0.008$ & $<0.008$ & 0.015 & 0.033 & 0.04 \\
Copper & 6.1 & 0.58 & 1.2 & 0.9 & 5.8 & 0.9 \\
Iron & $<5$ & $<5$ & $<5$ & 13 & 8 & $<5$ \\
Lead & 1.5 & 0.07 & 0.28 & 0.23 & 0.46 & $<0.06$ \\
Lithium & 2.1 & 0.39 & 3 & 3 & 2.7 & 2.8 \\
Manganese & 0.14 & $<0.06$ & 0.28 & 0.67 & 0.82 & 0.7 \\
Molybdenum & $<0.09$ & $<0.09$ & $<0.09$ & $<0.09$ & $<0.09$ & $<0.09$ \\
Nickel & 0.12 & $<0.03$ & 0.043 & 0.14 & 2.2 & 1.1 \\
Strontium & 0.025 & 0.015 & 0.12 & 0.24 & 0.93 & 1.4 \\
Thallium & $<0.05$ & $<0.05$ & $<0.05$ & $<0.05$ & $<0.05$ & $<0.05$ \\
Uranium & $<0.06$ & $<0.06$ & $<0.06$ & $<0.06$ & $<0.06$ & $<0.06$ \\
Vanadium & $<0.07$ & $<0.07$ & $<0.07$ & $<0.07$ & $<0.07$ & $<0.07$ \\
Zinc & 7.8 & 0.61 & 2.3 & 0.88 & 1.5 & 1.2 \\
\hline & & & & & & \\
\hline
\end{tabular}


Table 10.-Major-element concentrations for field blanks collected during the July-August 1987, November-December 1987, May-June 1988, March-April 1989, June 1989, February-March 1990, and May-June 1990 cruises

$[<$, less than detection limit; $\mathbf{m g} / \mathrm{L}$, milligrams per liter ]

\begin{tabular}{|c|c|c|c|c|}
\hline Biank type & $\begin{array}{l}\text { Calcium } \\
\text { (mg/l) }\end{array}$ & $\begin{array}{c}\text { Magnesium } \\
\text { (mg/l) }\end{array}$ & $\begin{array}{l}\text { Sodium } \\
\text { (mg/L) }\end{array}$ & $\begin{array}{l}\text { Sillca } \\
\text { (mg/L) }\end{array}$ \\
\hline \multicolumn{5}{|c|}{ August 9, 1987} \\
\hline Teflon-lined tank & 0.0052 & $<0.001$ & $<0.01$ & $<0.04$ \\
\hline Unlined tank & 0.0044 & 0.0012 & $<0.01$ & $<0.04$ \\
\hline \multicolumn{5}{|c|}{ December 6, 1987} \\
\hline Deionized Water & 3.6 & 1.2 & 2.5 & 0.76 \\
\hline \multicolumn{5}{|c|}{ December 13, 1987} \\
\hline Deionized Water & 1.9 & 0.53 & 4.6 & 1.6 \\
\hline \multicolumn{5}{|c|}{ December 19, 1987} \\
\hline Deionized Water & 0.0029 & $<0.001$ & $<0.01$ & 0.7 \\
\hline Teflon sampler bag & 0.24 & 0.18 & 1.7 & 1.5 \\
\hline Sieve & 0.23 & 0.18 & 1.7 & 1.5 \\
\hline Splitter & 0.44 & 0.34 & 3.1 & 1.9 \\
\hline Centrifuge effluent & 1 & 0.52 & 3.4 & 2 \\
\hline Ultrafilter permeate & 1 & 0.52 & 3.4 & 1.9 \\
\hline \multicolumn{5}{|c|}{ May 15,1988} \\
\hline Deionized Water & 0.028 & 0.16 & 0.05 & $<0.04$ \\
\hline \multicolumn{5}{|c|}{ May 18,1988} \\
\hline Teflon bag $\mathrm{A}$ & 0.0021 & $<0.001$ & $<0.01$ & $<0.04$ \\
\hline Teflon bag B & $<0.001$ & $<0.001$ & $<0.01$ & 0.048 \\
\hline Splitter A & 0.018 & 0.0039 & $<0.01$ & $<0.04$ \\
\hline Splitter B & 0.035 & 0.17 & 0.053 & $<0.04$ \\
\hline Sieve $\mathrm{A}$ & 0.0078 & $<0.001$ & $<0.01$ & $<0.04$ \\
\hline Sieve B & 0.0044 & $<0.001$ & $<0.01$ & $<0.04$ \\
\hline Splitter composite & 0.03 & 0.0091 & $<0.01$ & 0.049 \\
\hline Centrifuge effluent & 0.43 & 0.19 & 0.075 & 0.056 \\
\hline Ultrafilter retentate & 0.38 & 0.18 & 0.11 & $<0.04$ \\
\hline Ultrafilter permeate & 0.38 & 0.17 & 0.11 & $<0.04$ \\
\hline \multicolumn{5}{|c|}{$\underline{\text { May } 20,1988}$} \\
\hline Deionized Water & $<0.001$ & $<0.001$ & $<0.01$ & $<0.04$ \\
\hline \multicolumn{5}{|c|}{ May 31,1988} \\
\hline Deionized Water & 0.029 & 0.01 & 0.021 & 0.094 \\
\hline Process Blank ${ }^{1}$ & 0.024 & 0.0052 & 0.012 & 0.069 \\
\hline Process Blank ${ }^{2}$ & $<0.001$ & $<0.001$ & $<0.01$ & $<0.04$ \\
\hline Splitter' & 0.024 & 0.0057 & 0.016 & 0.096 \\
\hline Splitter ${ }^{2}$ & 0.024 & 0.0052 & 0.017 & 0.1 \\
\hline Centrifuge effluent ${ }^{1}$ & 0.13 & 0.034 & 0.038 & 0.078 \\
\hline Centrifuge effluent ${ }^{2}$ & 0.07 & 0.015 & 0.022 & 0.12 \\
\hline Ultrafilter permeate $^{1}$ & 0.16 & 0.07 & 0.1 & 0.19 \\
\hline Ultrafilter permeate ${ }^{2}$ & 0.06 & 0.016 & 0.021 & 0.11 \\
\hline \multicolumn{5}{|c|}{ June 8, 1988} \\
\hline Deionized Water & $<0.001$ & $<0.001$ & 0.01 & 0.054 \\
\hline Splitter ${ }^{1}$ & 0.014 & 0.0032 & 0.55 & 0.4 \\
\hline Centrifuge effluent ${ }^{1}$ & 0.58 & 0.2 & 0.84 & 0.46 \\
\hline Ultrafilter permeate $^{1}$ & 0.24 & 0.079 & 0.71 & 0.42 \\
\hline \multicolumn{5}{|c|}{ March 11, 1989} \\
\hline Deionized Water & 0.4 & 0.3 & 0.19 & 0.25 \\
\hline Teflon sampler bag & 0.83 & 0.64 & 0.43 & 0.56 \\
\hline Sieve & 0.79 & 0.62 & 0.4 & 0.54 \\
\hline Splitter & 0.76 & 0.59 & 0.39 & 0.54 \\
\hline Centrifuge effluent & 0.8 & 0.59 & 0.38 & 0.53 \\
\hline Ultrafilter permeate & 0.87 & 0.62 & 0.42 & 0.54 \\
\hline
\end{tabular}


Table 10.--Major-element concentrations for field blanks collected during the July-August 1987, November-December 1987, May-June 1988, March-April 1988, June 1989, February-March 1990, and May June 1990 cruises - Continued

\begin{tabular}{l} 
Blank type \\
\hline \\
Deionized Water \\
Teflon sampler bag \\
Sieve \\
Splitter \\
Centrifuge effluent \\
Ultrafilter permeate
\end{tabular}

Deionized Water

Teflon sampler bag

Sieve

Splitter

Centrifuge effluent

Ultrafilter permeate

Deionized Water

Teflon sampler bag

Sieve

Splitter

Centrifuge effluent

Ultrafilter permeate

Deionized Water

Teflon sampler bag

Sieve

Splitter

Centrifuge effluent

Ultrafilter permeate

Deionized Water

Teflon sampler bag

Sieve

Splitter

Centrifuge effluent

Ultrafilter permeate

Deionized Water

Nuclepore filter

Sieve

Splitter

Centrifuge effluent

Ultrafilter permeate

Calclum

Magneslum

Sodlum

(mgh)

Sllica

(mgh)

(mgl)

(mg/L)

$\begin{array}{lr}< & \text { March 25, 1989 } \\ <0.001 & <0.001 \\ 0.009 & 0.007 \\ 0.001 & 0.001 \\ 0.005 & 0.002 \\ 0.031 & 0.009 \\ 0.12 & 0.039\end{array}$

$\begin{array}{ll}0.022 & 0.38 \\ 2.5 & 0.84 \\ 2.5 & 0.84 \\ 2.8 & 0.81 \\ 2.8 & 0.81 \\ 2.7 & 0.83\end{array}$

\section{March 31, 1989}

$\begin{array}{llll}<0.001 & <0.001 & 0.11 & 0.048 \\ 0.23 & 0.16 & 4.3 & 0.82 \\ 0.23 & 0.16 & 4.3 & 0.81 \\ 0.3 & 0.21 & 5.3 & 1 \\ 0.3 & 0.2 & 5.3 & 1 \\ 0.32 & 0.21 & 5.3 & 1\end{array}$

June 6, 1989

$\begin{array}{ll}0.006 & 0.001 \\ 4.1 & 1.1 \\ 3.9 & 1.1 \\ 3 & 0.85 \\ 3.3 & 0.94 \\ 3.3 & 0.95\end{array}$

$\begin{array}{ll}0.041 & 0.073 \\ 1.7 & 0.37 \\ 1.9 & 0.9 \\ 1.7 & 0.6 \\ 1.9 & 0.7 \\ 2 & 0.79\end{array}$

June 21, 1989

$\begin{array}{llll}1.1 & 0.41 & 6.5 & 1.2 \\ 0.7 & 0.26 & 4.2 & 0.77 \\ 0.71 & 0.27 & 4.3 & 0.81 \\ 0.86 & 0.32 & 5 & 1 \\ 0.96 & 0.35 & 5.3 & 1.1 \\ 0.92 & 0.34 & 5.2 & 1\end{array}$

June 27, 1989

$\begin{array}{llll}0.46 & 0.28 & 9.1 & 1.8 \\ 0.42 & 0.25 & 8 & 1.6 \\ 0.41 & 0.25 & 8 & 1.5 \\ 0.59 & 0.36 & 9.2 & 1.9 \\ 0.6 & 0.36 & 9.4 & 1.8 \\ 0.62 & 0.36 & 9.4 & 1.9\end{array}$

March 13,1990

$\begin{array}{llll}0.004 & 0.002 & 12 & 4 \\ 0.011 & 0.002 & 14 & 4.4 \\ 0.005 & 0.002 & 12 & 3.8 \\ 0.084 & 0.024 & 7.1 & 2.3 \\ 0.077 & 0.022 & 7 & 2.2 \\ 0.26 & 0.071 & 7.1 & 2.3\end{array}$

Deionized Water

Nuclepore filter

Sieve

Splitter

Centrifuge effluent

Ultrafilter permeate

June 26, 1990

${ }_{2}$ Processed using unstained Teflon-coated churn splitter

\begin{tabular}{rrrl}
0.002 & $<0.001$ & 7.6 & 2.7 \\
$<0.001$ & $<0.001$ & 1.8 & 0.54 \\
0.006 & 0.001 & 12 & 3.6 \\
0.03 & 0.011 & 12 & 3.5 \\
0.24 & 0.061 & 12 & 3.4 \\
0.28 & 0.084 & 12 & 3.5 \\
\hline
\end{tabular}

${ }^{2}$ Processed using rust stained Teflon-coated churn splitters. 
Table 11.-- Quality-control data for analyses of samples from the July-August 1987 cruise

\begin{tabular}{|c|c|c|c|c|}
\hline Analyte & $\begin{array}{c}\text { Published } \\
\text { mean }\end{array}$ & $\begin{array}{l}\text { Published } \\
\text { standard } \\
\text { deviation }\end{array}$ & $\begin{array}{c}\text { Analytical } \\
\text { mean }\end{array}$ & $\begin{array}{l}\text { Analytical } \\
\text { standard } \\
\text { deviation }\end{array}$ \\
\hline \multicolumn{5}{|c|}{ U.S. Geological Survey's Standard Reference Water Sample T93 } \\
\hline Aluminum & 172 & 44 & 164 & 9.2 \\
\hline Arsenic & 5.6 & 1.6 & 5.8 & 0.27 \\
\hline Barium & 68.6 & 12 & 67 & 2.9 \\
\hline Beryllium & 7.2 & 1.5 & 6.3 & 0.23 \\
\hline Cadmium & 4.8 & 0.6 & 5.5 & 0.29 \\
\hline Chromium & 9.8 & 2.8 & 5.3 & 4.6 \\
\hline Cobalt & 5 & 1.2 & 5.7 & 0.34 \\
\hline Copper & 30.6 & 3.6 & 31 & 1.4 \\
\hline Lead & 11.2 & 3.5 & 11 & 0.37 \\
\hline Lithium & 21.6 & 2.4 & 22 & 0.57 \\
\hline Manganese & 99 & 4 & 101 & 5.1 \\
\hline Molybdenum & 19.4 & 2.7 & 19 & 1 \\
\hline Nickel & 11.8 & 3 & 19 & 1.9 \\
\hline Strontium & 64 & 3 & 67 & 2 \\
\hline Thallium & 2.1 & 0.6 & 2.3 & 0.3 \\
\hline Zinc & 27.8 & 4.4 & 28 & 1.6 \\
\hline \multicolumn{5}{|c|}{ U.S. Geological Survey's Standard Reference Water Sample T $99^{2}$} \\
\hline Calcium & 202 & 15 & 200 & 1.6 \\
\hline Iron & 0.137 & 0.029 & 0.13 & 0.006 \\
\hline Magnesium & 113 & 7 & 110 & 1.7 \\
\hline Silica & 10.9 & 0.7 & 11 & 0.15 \\
\hline Sodium & 323 & 17 & 320 & 3.2 \\
\hline Strontium & 3.9 & 0.2 & 3.8 & 0.038 \\
\hline \multicolumn{5}{|c|}{ U.S. Environmental Protection Agency Survey Sample $1085^{3}$} \\
\hline Chloride & 8.3 & 0.97 & 7.9 & 0.17 \\
\hline Nitrate (as nitrogen) & 2.0 & 0.1 & 2.0 & 0.0 \\
\hline Sulfate & 33.8 & 1.68 & 34.1 & 0.14 \\
\hline \multicolumn{5}{|c|}{ U.S. Geological Survey's Standard Reference Water Sample M2 ${ }^{4}$} \\
\hline Bicarbonate & 170 & 1.2 & 171 & 0.4 \\
\hline Carbonate & 1.7 & 0.01 & 1.7 & 0.0 \\
\hline
\end{tabular}

${ }^{1}$ Inductively-coupled mass spectrometry data, sample population of 6 ; concentrations are in micrograms per liter ${ }^{2}$ Inductively-coupled atomic emission spectroscopy data, sample population of 11 ; concentrations are in milligrams per liter

${ }^{3}$ Ion chromatography data, sample population of 9 ; concentrations are in milligrams per liter

${ }^{4}$ Alkalinity data, sample population of 6 ; concentrations are in milligrams per liter 
Table 12.-- Quality-control data for analyses of samples from the November-December 1987 cruise

\begin{tabular}{|c|c|c|c|c|}
\hline Analyte & $\begin{array}{c}\text { Published } \\
\text { mean }\end{array}$ & $\begin{array}{l}\text { Published } \\
\text { standard } \\
\text { deviation }\end{array}$ & $\begin{array}{c}\text { Analytical } \\
\text { mean }\end{array}$ & $\begin{array}{l}\text { Analytical } \\
\text { standard } \\
\text { deviation }\end{array}$ \\
\hline \multicolumn{5}{|c|}{ U.S. Geological Survey's Standard Reference Water Sample T93 ${ }^{1}$} \\
\hline Aluminum & 172 & 44 & 160 & 9 \\
\hline Arsenic & 5.6 & 1.6 & 5.2 & 0.3 \\
\hline Barium & 68.6 & 12 & 67 & 2.7 \\
\hline Beryllium & 7.2 & 1.5 & 6.1 & 0.6 \\
\hline Cadmium & 4.8 & 0.6 & 5.2 & 0.28 \\
\hline Chromium & 9.8 & 2.8 & 11 & 0.53 \\
\hline Cobalt & 5 & 1.2 & 5.5 & 0.44 \\
\hline Copper & 30.6 & 3.6 & 30 & 1.4 \\
\hline Lead & 11.2 & 3.5 & 11 & 0.95 \\
\hline Lithium & 21.6 & 2.4 & 22 & 0.91 \\
\hline Manganese & 99 & 4 & 100 & 4.3 \\
\hline Molybdenum & 19.4 & 2.7 & 18 & 0.5 \\
\hline Nickel & 11.8 & 3 & 20 & 1 \\
\hline Strontium & 64 & 3 & 64 & 2.3 \\
\hline Thallium & 2.1 & 0.6 & 2 & 0.21 \\
\hline Zinc & 27.8 & 4.4 & 27 & 1.1 \\
\hline \multicolumn{5}{|c|}{ U.S. Geological Survey's Standard Reference Water Sample T99² } \\
\hline Calcium & 202 & 15 & 200 & 1.3 \\
\hline Iron & 0.137 & 0.029 & 0.14 & 0.002 \\
\hline Magnesium & 113 & 7 & 110 & 0.74 \\
\hline Silica & 10.9 & 0.7 & 11 & 0.05 \\
\hline Sodium & 323 & 17 & 320 & 3.7 \\
\hline Strontium & 3.9 & 0.2 & 3.8 & 0.023 \\
\hline \multicolumn{5}{|c|}{ U.S. Environmental Protection Agency Survey Sample $1085^{3}$} \\
\hline Chloride & 8.3 & 0.97 & 7.92 & 0.17 \\
\hline Nitrate (as nitrogen) & 2.0 & 0.1 & 2.1 & 0.0 \\
\hline Sulfate & 33.8 & 1.68 & 34.0 & 0.13 \\
\hline \multicolumn{5}{|c|}{ U.S. Geological Survey's Standard Reference Water Sample M2 ${ }^{4}$} \\
\hline Bicarbonate & 170 & 1.2 & 171 & 1.6 \\
\hline Carbonate & 1.7 & 0.01 & 0.5 & 0.3 \\
\hline
\end{tabular}

${ }^{1}$ Inductively-coupled mass spectrometry data, sample population of 18 ; concentrations are in micrograms per liter

${ }^{2}$ Inductively-coupled atomic emission spectroscopy data, sample population of 11; concentrations are in milligrams per liter

${ }^{3}$ Ion chromatography data, sample population of 14 ; concentrations are in milligrams per liter

${ }^{4}$ Alkalinity data, sample population of 7 ; concentrations are in milligrams per liter 
Table 13.- Quality control data for analyses of samples from the May-June 1988 cruise

\begin{tabular}{|c|c|c|c|c|}
\hline Analyte & $\begin{array}{c}\text { Published } \\
\text { mean }\end{array}$ & $\begin{array}{l}\text { Published } \\
\text { standard } \\
\text { deviation }\end{array}$ & $\begin{array}{c}\text { Analytical } \\
\text { mean }\end{array}$ & $\begin{array}{l}\text { Analytical } \\
\text { standard } \\
\text { deviation }\end{array}$ \\
\hline \multicolumn{5}{|c|}{ U.S. Geological Survey's Standard Reference Water Sample T93 } \\
\hline Aluminum & 172 & 44 & 160 & 11 \\
\hline Arsenic & 5.6 & 1.6 & 5.5 & 0.39 \\
\hline Barium & 68.6 & 12 & 70 & 3.5 \\
\hline Beryllium & 7.2 & 1.5 & 6.3 & 0.35 \\
\hline Cadmium & 4.8 & 0.6 & 5.6 & 0.27 \\
\hline Chromium & 9.8 & 2.8 & 10 & 0.69 \\
\hline Cobalt & 5 & 1.2 & 5.7 & 0.36 \\
\hline Copper & 30.6 & 3.6 & 31 & 1.8 \\
\hline Lead & 11.2 & 3.5 & 12 & 0.37 \\
\hline Lithium & 21.6 & 2.4 & 21 & 1.1 \\
\hline Manganese & 99 & 4 & 99 & 6 \\
\hline Molybdenum & 19.4 & 2.7 & 18 & 0.51 \\
\hline Nickel & 11.8 & 3 & 19 & 1.3 \\
\hline Strontium & 64 & 3 & 64 & 4.1 \\
\hline Thallium & 2.1 & 0.6 & 2.3 & 0.12 \\
\hline Zinc & 27.8 & 4.4 & 27 & 1.9 \\
\hline \multicolumn{5}{|c|}{ U.S. Geological Survey's Standard Reference Water Sample T $99^{2}$} \\
\hline Calcium & 202 & 15 & 200 & 3 \\
\hline Iron & 0.137 & 0.029 & 0.13 & 0.006 \\
\hline Magnesium & 113 & 7 & 110 & 2.9 \\
\hline Silica & 10.9 & 0.7 & 11 & 0.2 \\
\hline Sodiunı & 323 & 17 & 320 & 20 \\
\hline Strontium & 3.9 & 0.2 & 3.8 & 0.092 \\
\hline \multicolumn{5}{|c|}{ U.S. Environmental Protection Agency Survey Sample $1085^{3}$} \\
\hline Chloride & 8.3 & 0.97 & 8.0 & 0.15 \\
\hline Nitrate (as nitrogen) & 2.0 & 0.1 & 2.1 & 0.0 \\
\hline Sulfate & 33.8 & 1.68 & 34.0 & 0.12 \\
\hline \multicolumn{5}{|c|}{ U.S. Geological Survey's Standard Reference Water Sample M2 ${ }^{4}$} \\
\hline Bicarbonate & 170 & 1.2 & 172 & 0.7 \\
\hline Carbonate & 1.7 & 0.01 & 1.3 & 0.4 \\
\hline
\end{tabular}

${ }^{1}$ Inductively-coupled mass spectrometry data, sample population of 19 ; concentrations are in micrograms per liter

${ }^{2}$ Inductively-coupled atomic emission spectroscopy data, sample population of 15 ; concentrations are in milligrams per liter

${ }^{3}$ Ion chromatography data, sample population of 11 ; concentrations are in milligrams per liter

${ }^{4}$ Alkalinity data, sample population of 4 ; concentrations are in milligrams per liter 
Table 14.- Quality-control data for analyses of samples from the March-April 1989 cruise

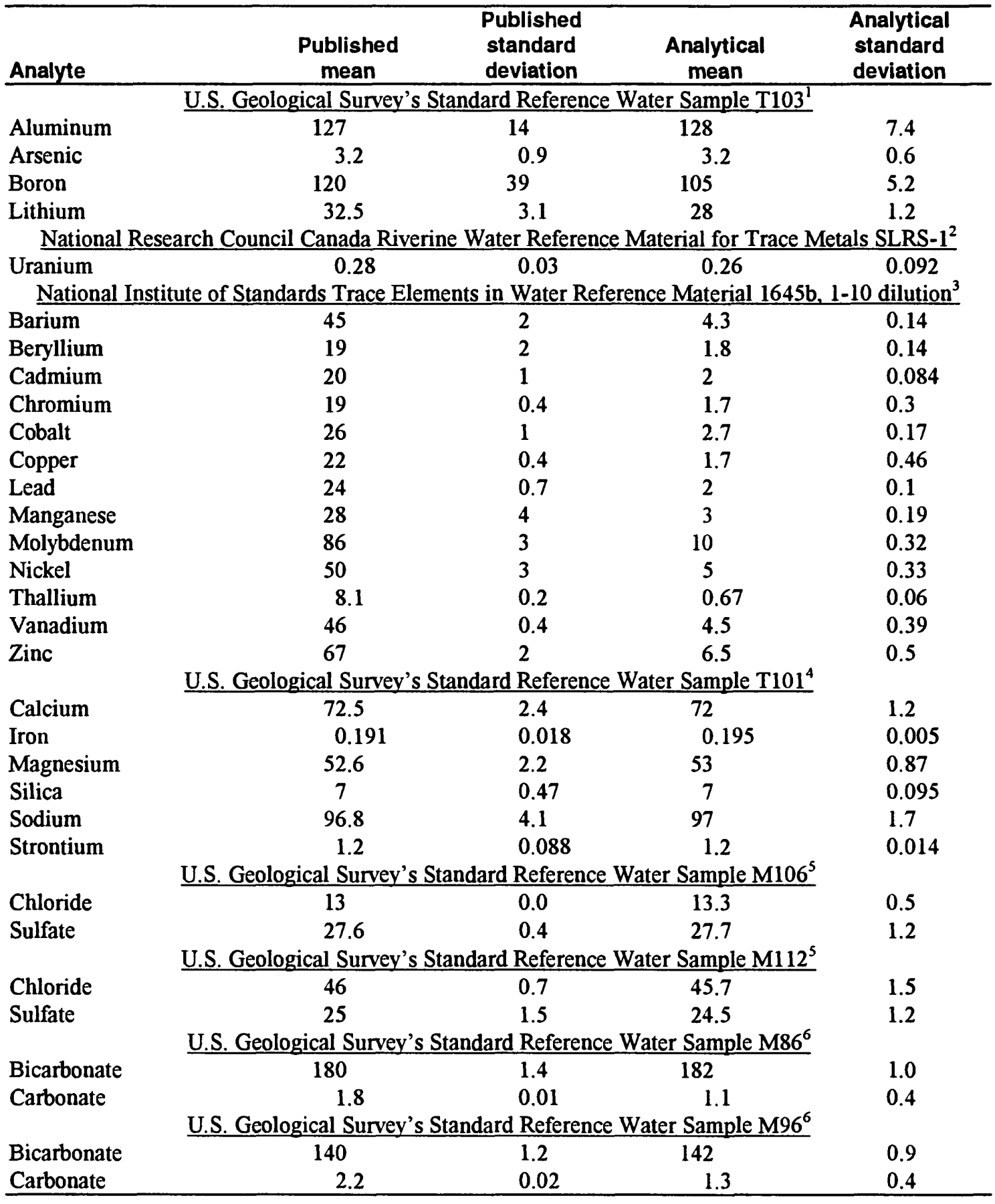

\footnotetext{
${ }^{1}$ Inductively-coupled mass spectrometry data, sample population of 11; concentrations are in micrograms per liter ${ }^{2}$ Inductively-coupled mass spectrometry data, sample population of 13; concentrations are in micrograms per liter ${ }^{3}$ Inductively-coupled mass spectrometry data, sample population of 10; concentrations are in micrograms per liter ${ }^{4}$ Inductively-coupled atomic emission spectroscopy data, sample population of 33 ; concentrations are in milligrams per liter ${ }^{5}$ Ion chromatography data, sample population of 13; concentrations are in milligrams per liter

${ }^{6}$ Alkalinity data, sample population of 14 ; concentrations are in milligrams per liter
} 
Table 15.- Quality-control data for analyses of samples from the June 1989 cruise

\begin{tabular}{|c|c|c|c|c|}
\hline Analyte & Publlshed mean & $\begin{array}{c}\text { Publlshed standard } \\
\text { devlation } \\
\end{array}$ & Analytlcal mean & $\begin{array}{c}\text { Analyticai standard } \\
\text { devlation }\end{array}$ \\
\hline \multicolumn{5}{|c|}{ U.S. Geological Survey's Standard Reference Water Sample T103' } \\
\hline Aluminum & 127 & 14 & 136 & 10 \\
\hline Arsenic & 3.2 & 0.9 & 3.7 & 0.41 \\
\hline Boron & 120 & 39 & 108 & 12 \\
\hline Lithium & 32.5 & 3.1 & 29 & 2.3 \\
\hline \multicolumn{5}{|c|}{ National Research Council Canada Riverine Water Reference Material for Trace Metals SLRS-1 ${ }^{2}$} \\
\hline $\begin{array}{l}\text { Uranium } \\
\text { National In }\end{array}$ & $\begin{array}{c}0.28 \\
\text { dards Trace Ele }\end{array}$ & & National Institute of Standards Trace Elements in Water Reference Material 1645b, 1-10 dilution ${ }^{3}$ & $\begin{array}{c}0.073 \\
\text { b, 1-10 dilution }\end{array}$ \\
\hline Barium & 45 & 2 & 4.3 & 0.23 \\
\hline Beryllium & 19 & 2 & 1.8 & 0.16 \\
\hline Cadmium & 20 & 1 & 2.1 & 0.15 \\
\hline Chromium & 19 & 0.4 & 1.9 & 0.64 \\
\hline Cobalt & 26 & 1 & 2.8 & 0.26 \\
\hline Copper & 22 & 0.4 & 2 & 0.18 \\
\hline Lead & 24 & 0.7 & 2.1 & 0.13 \\
\hline Manganese & 28 & 4 & 3 & 0.29 \\
\hline Molybdenum & 86 & 3 & 10 & 0.43 \\
\hline Nickel & 50 & 3 & 5.2 & 0.39 \\
\hline Thallium & 8.1 & 0.2 & 0.71 & 0.06 \\
\hline Vanadium & 46 & 0.4 & 4.6 & 0.47 \\
\hline Zinc & 67 & 2 & 7 & 0.63 \\
\hline \multicolumn{5}{|c|}{ U.S. Geological Survey's Standard Reference Water Sample T101 } \\
\hline Calcium & 72.5 & 2.4 & 72 & 2.9 \\
\hline Iron & 0.191 & 0.018 & 0.192 & 0.01 \\
\hline Magnesium & 52.6 & 2.2 & 55 & 2.3 \\
\hline Silica & 7 & 0.47 & 7.5 & 0.36 \\
\hline Sodium & 96.8 & 4.1 & 97 & 3.5 \\
\hline Strontium & 1.2 & 0.088 & 1.2 & 0.046 \\
\hline \multicolumn{5}{|c|}{ U.S. Geological Survey's Standard Reference Water Sample M106 ${ }^{5}$} \\
\hline Chloride & 13 & 0.0 & 13.1 & 0.3 \\
\hline Sulfate & 27.6 & 0.4 & 27.7 & 0.5 \\
\hline \multicolumn{5}{|c|}{ U.S. Geological Survey's Standard Reference Water Sample M112 } \\
\hline Chloride & 46 & 0.7 & 47.2 & 1.2 \\
\hline Sulfate & 25 & 1.5 & 24.6 & 0.4 \\
\hline \multicolumn{5}{|c|}{ U.S. Geological Survey's Standard Reference Water Sample M86 } \\
\hline Bicarbonate & 180 & 1.4 & 182 & 0.8 \\
\hline Carbonate & 1.8 & 0.01 & 1.8 & 0.01 \\
\hline \multicolumn{5}{|c|}{ U.S. Geological Survey's Standard Reference Water Sample M96 ${ }^{6}$} \\
\hline Bicarbonate & 140 & 1.2 & 141 & 1.0 \\
\hline Carbonate & 2.2 & 0.02 & 1.7 & 0.6 \\
\hline
\end{tabular}

\footnotetext{
'Inductively-coupled mass spectrometry data, sample population of 15; concentrations are in micrograms per liter ${ }^{2}$ Inductively-coupled mass spectrometry data, sample population of 13; concentrations are in micrograms per liter ${ }^{3}$ Inductively-coupled mass spectrometry data, sample population of 11 ; concentrations are in micrograms per liter ${ }^{4}$ Inductively-coupled atomic emission spectroscopy data, sample population of 24 ; concentrations are in milligrams per liter 5on chromatography data, sample population of 14 ; concentrations are in milligrams per liter

${ }^{6}$ Alkalinity data, sample population of 6 ; concentrations are in milligrams per liter
} 
Table 16.- Quality-control data for analyses of samples from the February-March 1990 cruise

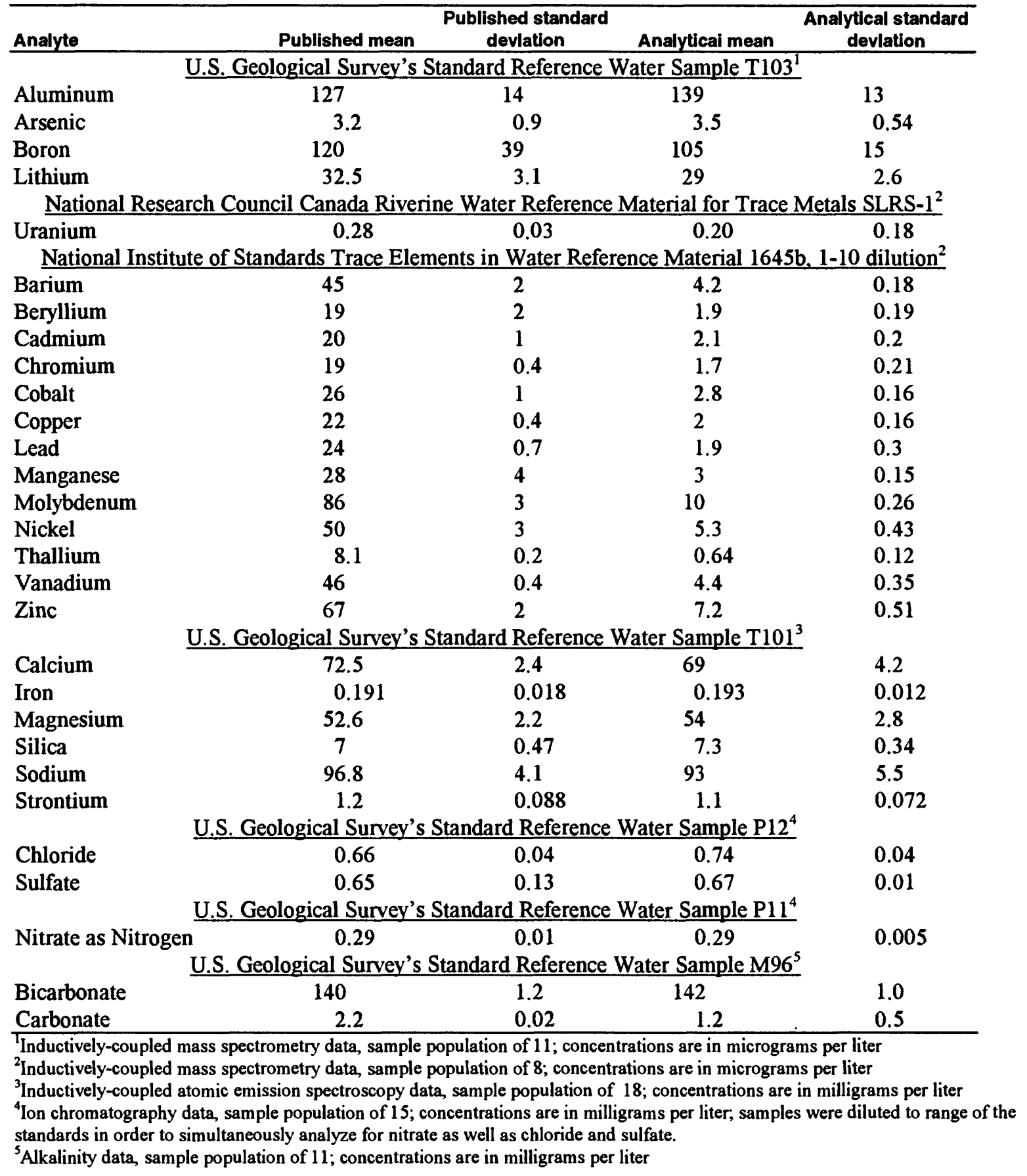


Table 17.- Quality-control data for analyses of samples from the May-June 1990 cruise

\begin{tabular}{|c|c|c|c|c|}
\hline Analyte & Publlshed mean & $\begin{array}{c}\text { Publlshed standard } \\
\text { devlatlon }\end{array}$ & Analytical mean & $\begin{array}{c}\text { Analytical standard } \\
\text { devlation }\end{array}$ \\
\hline \multicolumn{5}{|c|}{ U.S. Geological Survey's Standard Reference Water Sample T103' } \\
\hline Aluminum & 127 & 14 & 137 & 5 \\
\hline Arsenic & 3.2 & 0.9 & 3 & 0.52 \\
\hline Boron & 120 & 39 & 109 & 8 \\
\hline Lithium & 32.5 & 3.1 & 30 & 1.2 \\
\hline \multicolumn{5}{|c|}{ National Research Council Canada Riverine Water Reference Material for Trace Metals SLRS-1 ${ }^{2}$} \\
\hline \multicolumn{4}{|c|}{ National Institute of Standards Trace Elements in Water Reference Material $1645 \mathrm{~b}, 1-10$ dilution ${ }^{2}$} & $\begin{array}{c}0.073 \\
\text { b, } 1-10 \text { dilution }^{2}\end{array}$ \\
\hline Barium & 45 & 2 & 4.3 & 0.16 \\
\hline Beryllium & 19 & 2 & 1.9 & 0.18 \\
\hline Cadmium & 20 & 1 & 2.2 & 0.21 \\
\hline Chromium & 19 & 0.4 & 1.8 & 0.067 \\
\hline Cobalt & 26 & 1 & 2.9 & 0.09 \\
\hline Copper & 22 & 0.4 & 2 & 0.059 \\
\hline Lead & 24 & 0.7 & 2 & 0.097 \\
\hline Manganese & 28 & 4 & 3.2 & 0.094 \\
\hline Molybdenum & 86 & 3 & 10 & 0.37 \\
\hline Nickel & 50 & 3 & 5.3 & 0.26 \\
\hline Thallium & 8.1 & 0.2 & 0.72 & 0.061 \\
\hline Vanadium & 46 & 0.4 & 4.7 & 0.17 \\
\hline Zinc & 67 & 2 & 7.2 & 0.38 \\
\hline \multicolumn{5}{|c|}{ U.S. Geological Survey's Standard Reference Water Sample T107 } \\
\hline Calcium & 11.7 & 0.7 & 12 & 0.27 \\
\hline Iron & 0.052 & 0.007 & 0.053 & 0.004 \\
\hline Magnesium & 2.1 & 0.13 & 2.2 & 0.071 \\
\hline Silica & 7.7 & 0.5 & 8.3 & 0.15 \\
\hline Sodium & 20.7 & 1.1 & 23 & 0.52 \\
\hline Strontium & 0.061 & 0.004 & 0.064 & 0.002 \\
\hline \multicolumn{5}{|c|}{ U.S. Geological Survey's Standard Reference Water Sample P12 } \\
\hline Chloride & 0.66 & 0.04 & 0.68 & 0.02 \\
\hline Sulfate & 0.65 & 0.13 & 0.63 & 0.01 \\
\hline \multicolumn{5}{|c|}{ U.S. Geological Survey's Standard Reference Water Sample P11 ${ }^{4}$} \\
\hline Nitrate as Nitrogen & 0.29 & 0.01 & 0.28 & 0.005 \\
\hline \multicolumn{5}{|c|}{ U.S. Geological Survey's Standard Reference Water Sample M96 ${ }^{5}$} \\
\hline Bicarbonate & 140 & 1.2 & 141 & 1.3 \\
\hline Carbonate & 2.2 & 0.02 & 1.0 & 0.4 \\
\hline
\end{tabular}

'Inductively-coupled mass spectrometry data, sample population of 8; concentrations are in micrograms per liter

${ }^{2}$ Inductively-coupled mass spectrometry data, sample population of 6; concentrations are in micrograms per liter

${ }^{3}$ Inductively-coupled atomic emission spectroscopy data, sample population of 29 ; concentrations are in milligrams per liter

${ }^{4}$ Ion chromatography data, sample population of 12 ; concentrations are in milligrams per liter, samples were diluted to range of the standards in order to simultaneously analyze for nitrate as well as chloride and sulfate.

${ }^{5}$ Alkalinity data, sample population of 18 ; concentrations are in milligrams per liter 


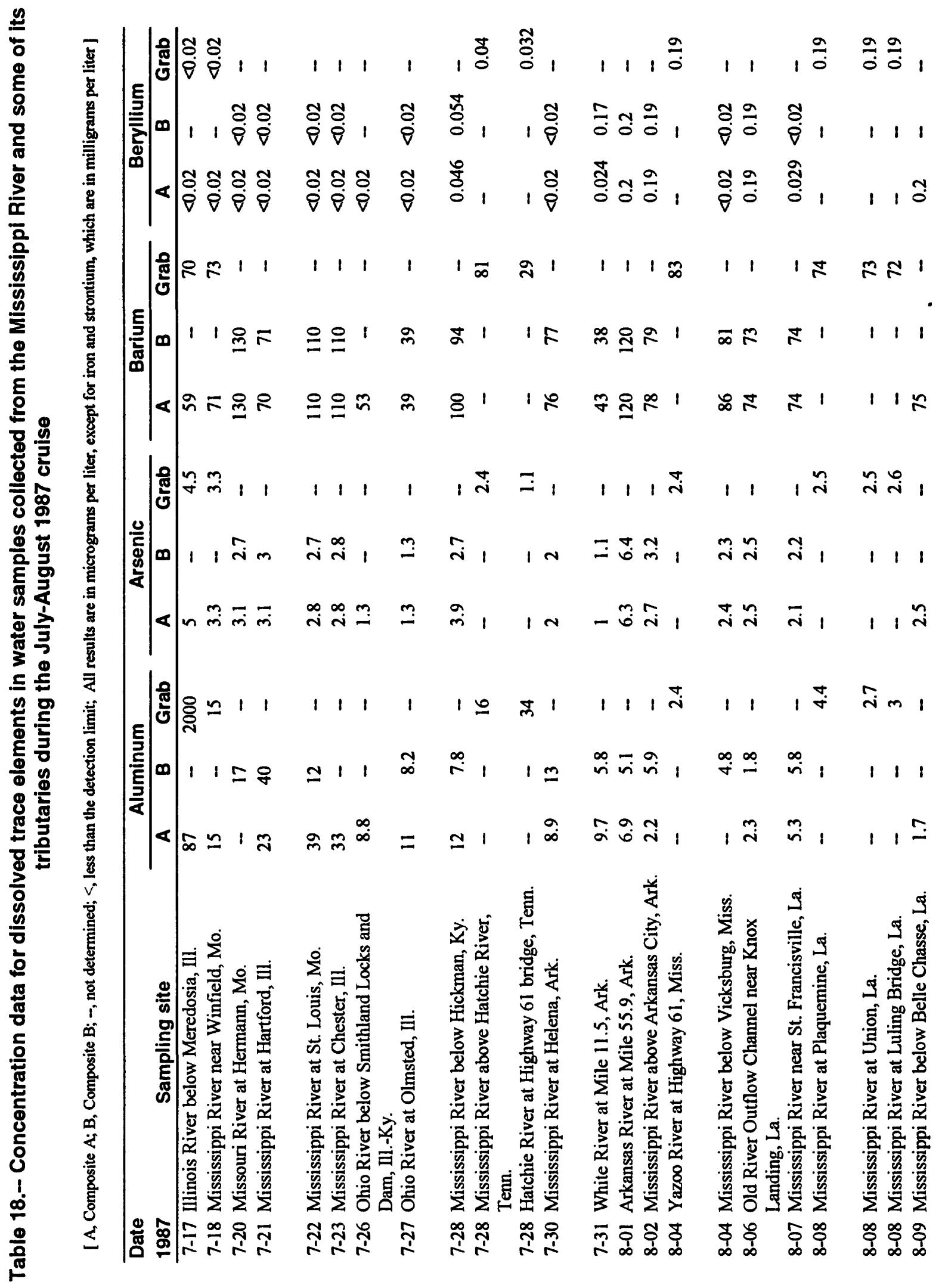




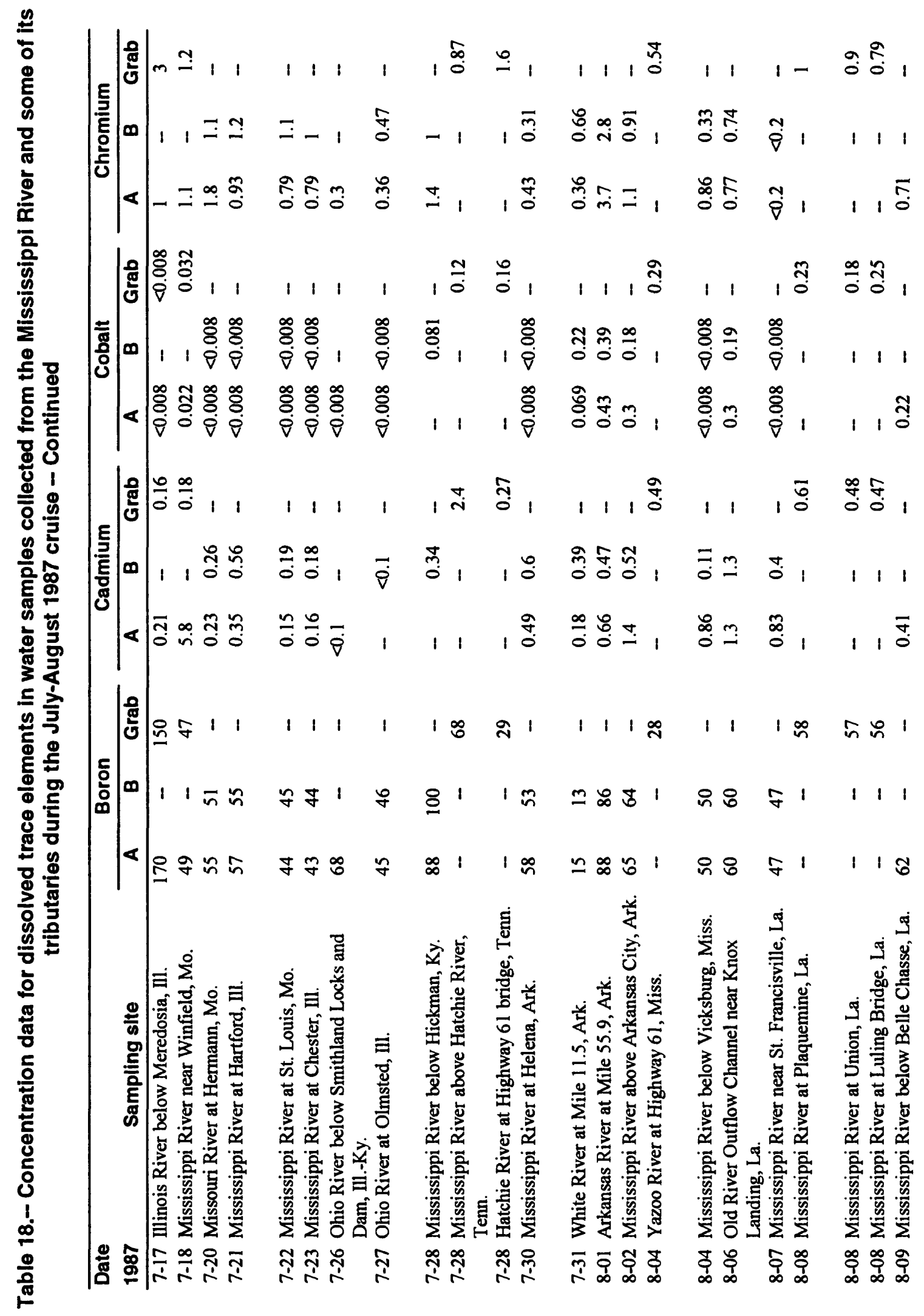




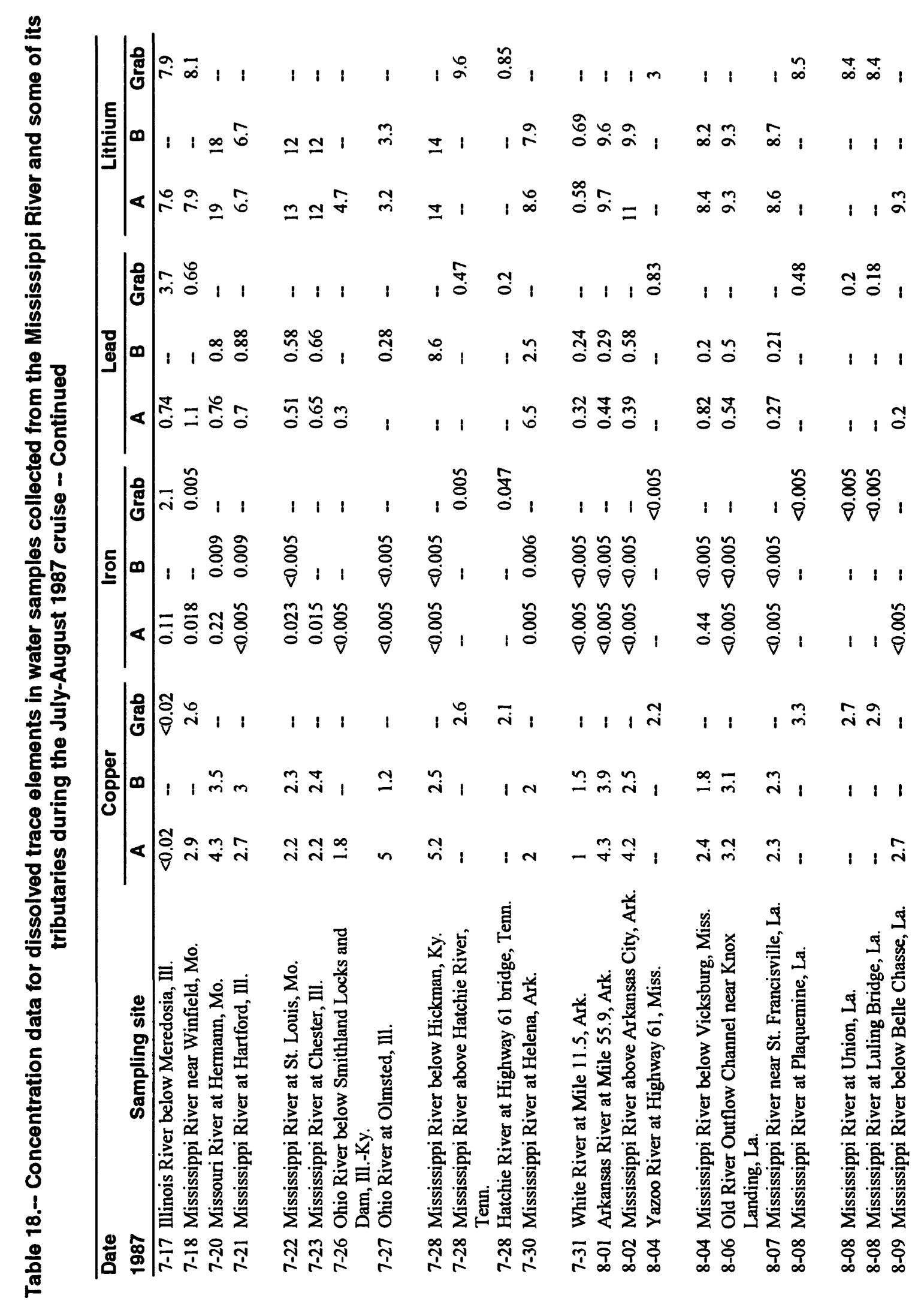

33 


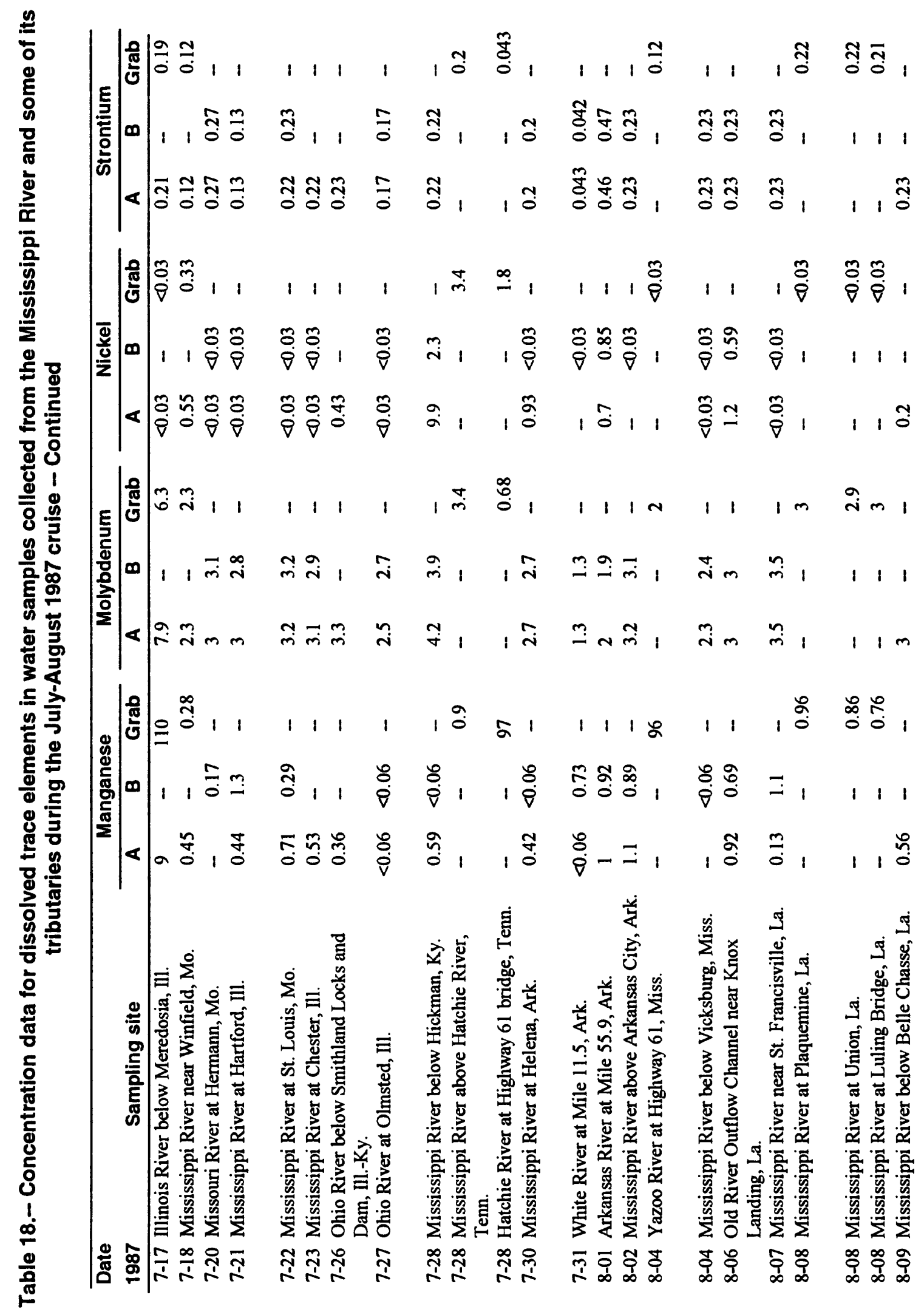




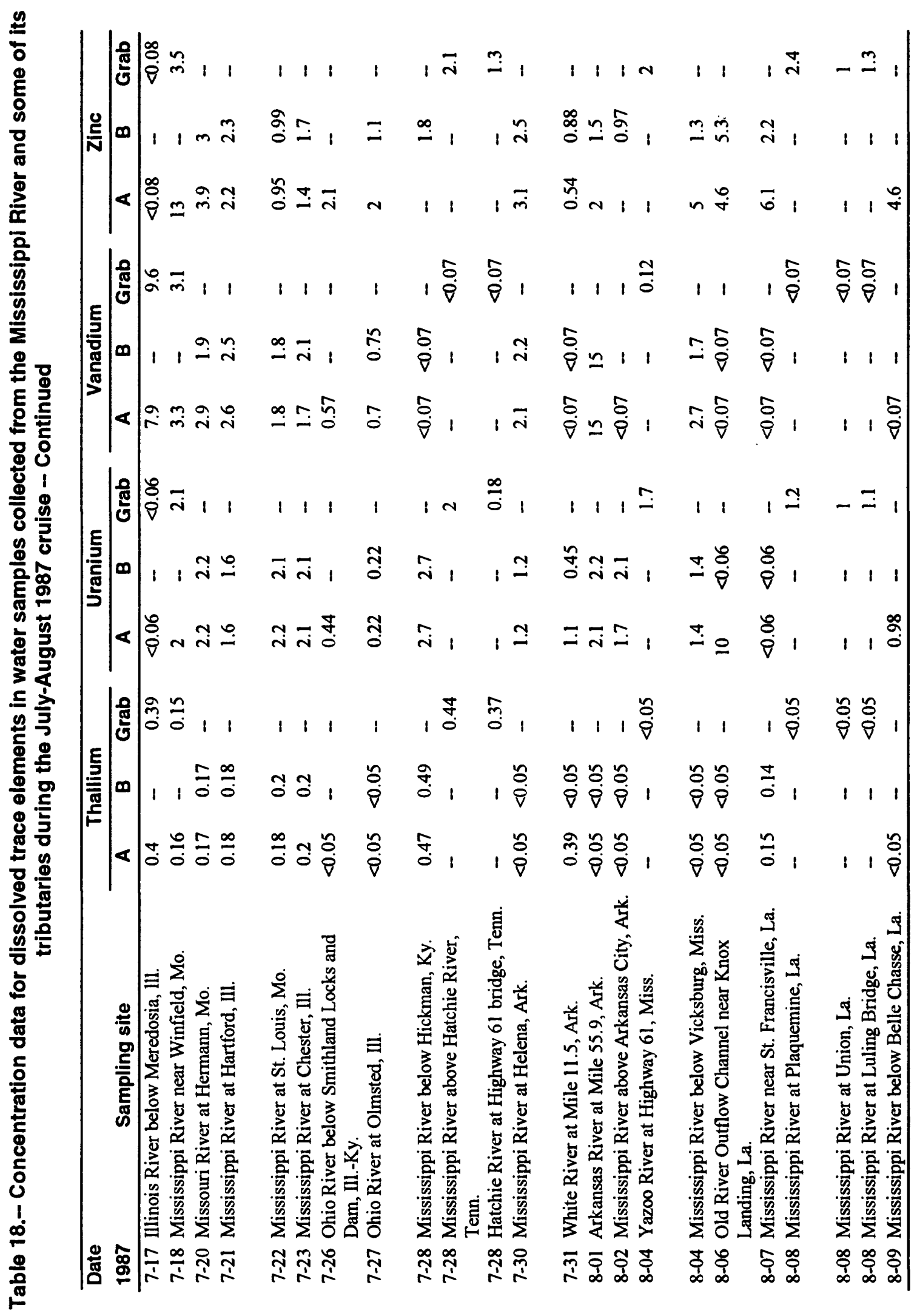


Table 19.- Concentration data for dissolved trace elements in water samples collected from the Mississippi River and some of its tributaries during the November-December 1987 cruise

[ A, Composite A; B, Composite B; --, not determined; <, less than the detection limit; All results are in micrograms per liter, except for iron and strontium, which are in milligrams per liter ]

\begin{tabular}{|c|c|c|c|c|c|c|c|c|c|}
\hline \multirow[b]{2}{*}{ Sampling site } & \multicolumn{3}{|c|}{ Aluminum } & \multicolumn{3}{|c|}{ Arsenic } & \multicolumn{3}{|c|}{ Barium } \\
\hline & A & B & Grab & A & B & Grab & $\mathbf{A}$ & B & Grab \\
\hline 11-29 Illinois River below Meredosia, Ill. & 6.7 & - & -- & 2.7 & - & - & 46 & - & - \\
\hline 11-30 Mississippi River near Winfield, Mo. & 7.3 & 7.8 & - & 1.2 & 1.2 & - & 46 & 46 & - \\
\hline 12-02 Missouri River at St. Charles, Mo. & 2.1 & 7.7 & - & 1.7 & 1.7 & - & 100 & 100 & - \\
\hline 12-03 Mississippi River at St. Louis, Mo. & 13 & 6.8 & - & 1.7 & 1.7 & - & 75 & 73 & - \\
\hline 12-05 Mississippi River at Thebes, Ill. & 30 & 24 & - & 1.6 & 1.7 & - & 79 & 78 & - \\
\hline 12-06 Ohio River at Olmsted, Ill. & 36 & 42 & - & 1.3 & 1.2 & - & 41 & 40 & - \\
\hline 12-07 Mississippi River below Hickman, Ky. & 5.6 & 8.8 & - & 1.2 & 1.2 & - & 61 & 63 & - \\
\hline 12-08 Mississippi River at Fulton, Tenn. & 1.6 & 4.3 & - & 1.3 & 1.4 & - & 65 & 68 & - \\
\hline 12-11 Mississippi River at Helena, Ark. & 13 & 15 & - & 0.85 & 0.92 & - & 66 & 67 & - \\
\hline 12-12 White River at Mile 11.5, Ark. & 14 & 10 & -- & $<0.6$ & $<0.6$ & -- & 40 & 40 & - \\
\hline 12-13 Mississippi River above Arkansas City, Ark. & $<0.2$ & $<0.2$ & -- & 1.6 & 1.8 & - & 70 & 73 & - \\
\hline 12-14 Yazoo River at Mile 10, Miss. & 43 & - & - & 1.1 & - & - & 31 & - & - \\
\hline 12-15 Mississippi River below Vicksburg, Miss. & 62 & 54 & - & 1.3 & 1.3 & -- & 70 & 73 & - \\
\hline $\begin{array}{l}\text { 12-17 Old River Outflow Channel near Knox } \\
\text { Landing, La. }\end{array}$ & 5.4 & 5.2 & - & 1.4 & 1.4 & - & 74 & 72 & - \\
\hline 12-18 Mississippi River near St. Francisville, La. & 3.1 & 20 & -- & 1.5 & 1.3 & -- & 72 & 72 & - \\
\hline 12-20 Mississippi River below Belle Chasse, La. & 16 & 22 & -- & 1.5 & 1.6 & _- & 71 & 75 & - \\
\hline
\end{tabular}

Table 19.- Concentration data for dissolved trace elements in water samples collected from the Mississippi River and some of its tributaries during the November-December 1987 cruise Continued

\begin{tabular}{|c|c|c|c|c|c|c|c|c|c|}
\hline \multirow[b]{2}{*}{ Sampling site } & \multicolumn{3}{|c|}{ Beryllium } & \multicolumn{3}{|c|}{ Boron } & \multicolumn{3}{|c|}{ Cadmium } \\
\hline & A & B & Grab & A & B & Grab & A & B & Grab \\
\hline 11-29 Illinois River below Meredosia, Ill. & $<0.02$ & - & -- & 140 & -- & - & 0.11 & - & - \\
\hline 11-30 Mississippi River near Winfield, Mo. & $<0.02$ & $<0.02$ & -- & 30 & 29 & - & $<0.1$ & $<0.1$ & - \\
\hline 12-02 Missouri River at St. Charles, Mo. & $<0.02$ & $<0.02$ & - & 73 & 70 & - & $<0.1$ & $<0.1$ & - \\
\hline 12-03 Mississippi River at St. Louis, Mo. & $<0.02$ & $<0.02$ & - & 63 & 59 & - & 0.39 & $<0.1$ & - \\
\hline 12-05 Mississippi River at Thebes, Ill. & $<0.02$ & $<0.02$ & -- & 53 & 50 & - & $<0.1$ & $<0.1$ & - \\
\hline 12-06 Ohio River at Olmsted, Ill. & $<0.02$ & $<0.02$ & - & 55 & 49 & - & 0.12 & $<0.1$ & - \\
\hline 12-07 Mississippi River below Hickman, Ky. & $<0.02$ & $<0.02$ & -- & 59 & 58 & - & 1.6 & $<0.1$ & - \\
\hline 12-08 Mississippi River at Fulton, Tenn. & $<0.02$ & $<0.02$ & - & 58 & 60 & - & $<0.1$ & $<0.1$ & - \\
\hline 12-11 Mississippi River at Helena, Ark. & $<0.02$ & $<0.02$ & - & 55 & 58 & - & $<0.1$ & $<0.1$ & - \\
\hline 12-12 White River at Mile 11.5, Ark. & $<0.02$ & $<0.02$ & - & 8.5 & 7.9 & - & $<0.1$ & $<0.1$ & - \\
\hline 12-13 Mississippi River above Arkansas City, Ark. & $<0.02$ & $<0.02$ & -- & 51 & 50 & -- & $<0.1$ & $<0.1$ & - \\
\hline 12-14 Yazoo River at Mile 10, Miss. & 0.068 & - & - & 18 & - & - & 0.64 & - & - \\
\hline 12-15 Mississippi River below Vicksburg, Miss. & $<0.02$ & $<0.02$ & - & 52 & 54 & - & 0.1 & 0.14 & - \\
\hline $\begin{array}{l}\text { 12-17 Old River Outflow Channel near Knox } \\
\text { Landing, La. }\end{array}$ & $<0.02$ & $<0.02$ & - & 54 & 52 & -- & 0.16 & $<0.1$ & - \\
\hline 12-18 Mississippi River near St. Francisville, La. & $<0.02$ & $<0.02$ & -- & 52 & 51 & - & $<0.1$ & 0.19 & - \\
\hline 12-20 Mississippi River below Belle Chasse, La. & $<0.02$ & $<0.02$ & - & 49 & 52 & - & 0.17 & $<0.1$ & - \\
\hline
\end{tabular}


Table 19.- Concentration data for dissolved trace elements in water samples collected from the Mississippi River and some of its tributaries during the November-December 1987 cruise Continued

\begin{tabular}{|c|c|c|c|c|c|c|c|c|c|}
\hline \multirow[b]{2}{*}{ Sampling site } & \multicolumn{3}{|c|}{ Chromium } & \multicolumn{3}{|c|}{ Cobalt } & \multicolumn{3}{|c|}{ Copper } \\
\hline & A & B & Grab & A & B & Grab & $\mathbf{A}$ & B & Grab \\
\hline 11-29 Illinois River below Meredosia, Ill. & 0.54 & - & - & $<0.008$ & - & - & 1.9 & - & - \\
\hline 11-30 Mississippi River near Winfield, Mo. & 0.32 & 0.44 & -- & $<0.008$ & $<0.008$ & - & 0.64 & 0.7 & -- \\
\hline 12-02 Missouri River at St. Charles, Mo. & $<0.2$ & 0.31 & - & $<0.008$ & $<0.008$ & - & 1 & 1.2 & - \\
\hline 12-03 Mississippi River at St. Louis, Mo. & 1.1 & 0.37 & - & $<0.008$ & $<0.008$ & - & 1.5 & 1 & - \\
\hline 12-05 Mississippi River at Thebes, Ill. & 0.45 & 0.37 & - & $<0.008$ & $<0.008$ & - & 1.2 & 0.98 & -- \\
\hline 12-06 Ohio River at Olmsted, Ill. & 0.58 & 0.64 & - & $<0.008$ & $<0.008$ & - & - & 1.5 & - \\
\hline 12-07 Mississippi River below Hickman, Ky. & 0.46 & 0.5 & - & $<0.008$ & $<0.008$ & - & 1.1 & 1 & _- \\
\hline 12-08 Mississippi River at Fulton, Tenn. & 0.34 & 0.41 & - & $<0.008$ & $<0.008$ & - & 1.1 & 0.94 & - \\
\hline 12-11 Mississippi River at Helena, Ark. & $<0.2$ & $<0.2$ & - & $<0.008$ & $<0.008$ & - & 0.6 & 0.6 & - \\
\hline 12-12 White River at Mile 11.5, Ark. & 0.27 & $<0.2$ & - & $<0.008$ & $<0.008$ & - & 0.73 & 0.44 & - \\
\hline 12-13 Mississippi River above Arkansas City, Ark. & 8.6 & 7.5 & - & $<0.008$ & $<0.008$ & - & 0.99 & 1 & - \\
\hline 12-14 Yazoo River at Mile 10, Miss. & 0.53 & - & - & 0.029 & - & - & 1.6 & - & - \\
\hline 12-15 Mississippi River below Vicksburg, Miss. & 0.97 & 0.59 & - & $<0.008$ & $<0.008$ & - & 1.3 & 1.8 & - \\
\hline $\begin{array}{l}\text { 12-17 Old River Outflow Channel near Knox } \\
\text { Landing, La. }\end{array}$ & 0.75 & 0.57 & - & $<0.008$ & $<0.008$ & - & 1.9 & 1.3 & - \\
\hline 12-18 Mississippi River near St. Francisville, La. & 0.62 & 0.54 & - & $<0.008$ & $<0.008$ & - & 1.2 & 1.8 & -- \\
\hline 12-20 Mississippi River below Belle Chasse, La. & 0.52 & 0.72 & - & $<0.008$ & $<0.008$ & - & 1.8 & 1.5 & - \\
\hline
\end{tabular}

Table 19.- Concentration data for dissolved trace elements in water samples collected from the Mississippi River and some of its tributaries during the November-December 1987 cruise -Continued

\begin{tabular}{|c|c|c|c|c|c|c|c|c|c|}
\hline \multirow{2}{*}{$\begin{array}{ll}\text { Date } & \\
1987 & \text { Sampling slte } \\
\end{array}$} & \multicolumn{3}{|c|}{ Iron } & \multicolumn{3}{|c|}{ Lead } & \multicolumn{3}{|c|}{ Llthlum } \\
\hline & A & B & Grab & A & B & Grab & A & B & Grab \\
\hline 11-29 Illinois River below Meredosia, Ill. & 0.006 & - & -- & 0.3 & -- & - & 6.9 & - & - \\
\hline 11-30 Mississippi River near Winfield, Mo. & 0.009 & 0.01 & -- & 0.12 & 0.087 & -- & 4 & 4.2 & - \\
\hline 12-02 Missouri River at St. Charles, Mo. & $<0.005$ & 0.008 & -- & $<0.06$ & 0.22 & - & 25 & 25 & - \\
\hline 12-03 Mississippi River at St. Louis, Mo. & 0.018 & 0.005 & -- & - & 0.092 & - & 15 & 15 & - \\
\hline 12-05 Mississippi River at Thebes, III. & 0.023 & 0.006 & - & 0.31 & 0.16 & - & 14 & 15 & -- \\
\hline 12-06 Ohio River at Olmsted, Ill. & 0.038 & 0.041 & - & - & 0.13 & -- & 6.2 & 5.9 & - \\
\hline 12-07 Mississippi River below Hickman, Ky. & 0.005 & $<0.005$ & - & 0.065 & $<0.06$ & - & 9 & 9.2 & - \\
\hline 12-08 Mississippi River at Fulton, Tenn. & $<0.005$ & $<0.005$ & - & 0.098 & 0.11 & - & 9.6 & 10 & - \\
\hline 12-11 Mississippi River at Helena, Ark. & 0.012 & 0.011 & -- & 0.27 & $<0.06$ & - & 9.3 & 9.9 & - \\
\hline 12-12 White River at Mile 11.5, Ark. & 0.016 & 0.009 & - & 0.27 & 0.32 & - & 0.33 & 0.32 & - \\
\hline 12-13 Mississippi River above Arkansas City, Ark. & $<0.005$ & $<0.005$ & - & $<0.06$ & $<0.06$ & - & 8.3 & 8.7 & - \\
\hline 12-14 Yazoo River at Mile 10, Miss. & 0.067 & 0.091 & - & 0.23 & - & -- & 0.81 & - & - \\
\hline 12-15 Mississippi River below Vicksburg, Miss. & 0.068 & 0.054 & -- & 0.18 & 0.24 & - & 7.6 & 7.9 & - \\
\hline $\begin{array}{l}\text { 12-17 Old River Outflow Channel near Knox } \\
\text { Landing, La. }\end{array}$ & 0.006 & $<0.005$ & - & 0.2 & 0.13 & - & 8 & 7.9 & - \\
\hline 12-18 Mississippi River near St. Francisville, La. & $<0.005$ & 0.014 & - & 0.08 & - & - & 8.1 & 8.2 & - \\
\hline 12-20 Mississippi River below Belle Chasse, La. & 0.01 & 0.012 & - & 0.33 & 0.18 & - & 8.4 & 8.7 & - \\
\hline
\end{tabular}


Table 19.-- Concentration data for dissolved trace elements in water samples collected from the Mississippi River and some of its tributaries during the November-December 1987 cruise Continued

\begin{tabular}{|c|c|c|c|c|c|c|c|c|c|}
\hline \multirow[b]{2}{*}{ Sampling site } & \multicolumn{3}{|c|}{ Manganese } & \multicolumn{3}{|c|}{ Molybdenum } & \multicolumn{3}{|c|}{ Nlckel } \\
\hline & A & B & Grab & A & B & Grab & A & B & Grab \\
\hline 11-29 Illinois River below Meredosia, Ill. & 0.41 & - & -- & 4.7 & -- & - & 7.6 & -- & - \\
\hline 11-30 Mississippi River near Winfield, Mo. & 1.2 & 2 & -- & 0.99 & 1 & - & 2.7 & 2.1 & - \\
\hline 12-02 Missouri River at St. Charles, Mo. & 0.7 & 0.79 & - & 2.4 & 2.2 & - & $<0.03$ & $<0.03$ & - \\
\hline 12-03 Mississippi River at St. Louis, Mo. & 0.67 & 0.39 & - & 1.8 & 1.8 & - & 3.5 & 4 & - \\
\hline 12-05 Mississippi River at Thebes, Ill. & 2.3 & 0.9 & - & 1.8 & 1.8 & - & 4.5 & 3.6 & - \\
\hline 12-06 Ohio River at Olmsted, Ill. & 5.7 & 6.7 & -- & 3.7 & 3.9 & - & 1.6 & $<0.03$ & - \\
\hline 12-07 Mississippi River below Hickman, Ky. & 0.73 & - & -- & 3 & 2.7 & - & 0.69 & 0.075 & - \\
\hline 12-08 Mississippi River at Fulton, Tenn. & 0.16 & 0.15 & -- & 2.7 & 2.9 & -- & $<0.03$ & 0.57 & -- \\
\hline 12-11 Mississippi River at Helena, Ark. & 1.1 & 1.2 & -- & 2.9 & 2.7 & - & $<0.03$ & $<0.03$ & - \\
\hline 12-12 White River at Mile 11.5, Ark. & 1.5 & 0.68 & - & 0.32 & 0.31 & - & $<0.03$ & 0.078 & - \\
\hline 12-13 Mississippi River above Arkansas City, Ark. & $<0.06$ & $<0.06$ & -- & 2.6 & 2.7 & - & 1.9 & 3.8 & - \\
\hline 12-14 Yazoo River at Mile 10, Miss. & 32 & - & - & 0.17 & - & - & 1.1 & - & - \\
\hline 12-15 Mississippi River below Vicksburg, Miss. & 3.6 & 2.9 & - & 2.3 & 2.3 & - & 0.68 & 2.2 & - \\
\hline $\begin{array}{l}\text { 12-17 Old River Outflow Channel near Knox } \\
\text { Landing, La. }\end{array}$ & 1.2 & 1 & - & 2.4 & 2.3 & - & 3.3 & 2.4 & - \\
\hline 12-18 Mississippi River near St. Francisville, La. & 0.1 & 0.74 & - & 2.4 & 2.4 & - & 1.8 & 2.5 & -- \\
\hline 12-20 Mississippi River below Belle Chasse, La. & 0.39 & 0.41 & -- & 2.3 & 2.3 & - & 3.4 & 4.2 & - \\
\hline
\end{tabular}

Table 19.-- Concentration data for dissolved trace elements in water samples collected from the Mississippi River and some of its tributaries during the November-December 1987 cruise -Continued

\begin{tabular}{|c|c|c|c|c|c|c|c|c|c|}
\hline \multirow{2}{*}{$\begin{array}{ll}\text { Date } & \\
1987 & \text { Sampling site } \\
\end{array}$} & \multicolumn{3}{|c|}{ Strontium } & \multicolumn{3}{|c|}{ Thallium } & \multicolumn{3}{|c|}{ Uranlum } \\
\hline & $\bar{A}$ & B & Grab & A & B & Grab & $\mathbf{A}$ & B & Grab \\
\hline 11-29 Illinois River below Meredosia, Ill. & 0.26 & - & -- & $<0.05$ & - & - & 0.88 & - & - \\
\hline 11-30 Mississippi River near Winfield, Mo. & 0.09 & 0.097 & -- & $<0.05$ & $<0.05$ & - & 0.9 & 1 & -- \\
\hline 12-02 Missouri River at St. Charles, Mo. & 0.39 & 0.38 & -- & $<0.05$ & $<0.05$ & -- & 4 & 3.6 & - \\
\hline 12-03 Mississippi River at St. Louis, Mo. & 0.24 & 0.23 & -- & $<0.05$ & $<0.05$ & - & 2 & 2 & - \\
\hline 12-05 Mississippi River at Thebes, IIl. & 0.22 & 0.23 & - & $<0.05$ & $<0.05$ & - & 2 & 2 & -- \\
\hline 12-06 Ohio River at Olmsted, Ill. & 0.2 & 0.2 & - & $<0.05$ & $<0.05$ & - & 0.28 & 0.28 & - \\
\hline 12-07 Mississippi River below Hickman, Ky. & 0.22 & 0.22 & -- & $<0.05$ & $<0.05$ & - & 1.3 & 1.4 & - \\
\hline 12-08 Mississippi River at Fulton, Tenn. & 0.22 & 0.22 & - & $<0.05$ & $<0.05$ & - & 1.6 & 1.6 & - \\
\hline 12-11 Mississippi River at Helena, Ark. & 0.22 & 0.21 & - & $<0.05$ & $<0.05$ & - & 1.3 & 1.3 & - \\
\hline 12-12 White River at Mile 11.5, Ark. & 0.036 & 0.036 & -- & $<0.05$ & $<0.05$ & - & 0.31 & 0.24 & - \\
\hline 12-13 Mississippi River above Arkansas City, Ark. & 0.21 & 0.21 & - & $<0.05$ & $<0.05$ & - & 1.4 & 1.4 & _- \\
\hline 12-14 Yazoo River at Mile 10, Miss. & 0.05 & 0.05 & - & $<0.05$ & - & - & $<0.06$ & - & - \\
\hline 12-15 Mississippi River below Vicksburg, Miss. & 0.2 & 0.2 & -- & $<0.05$ & $<0.05$ & - & 1.5 & 1.4 & - \\
\hline $\begin{array}{l}\text { 12-17 Old River Outflow Channel near Knox } \\
\text { Landing, La. }\end{array}$ & 0.2 & 0.2 & - & $<0.05$ & $<0.05$ & - & 1.3 & 1.4 & - \\
\hline 12-18 Mississippi River near St. Francisville, La. & 0.21 & 0.21 & - & $<0.05$ & $<0.05$ & - & 1.4 & 1.3 & - \\
\hline 12-20 Mississippi River below Belle Chasse, La. & 0.2 & 0.2 & - & $<0.05$ & $<0.05$ & -- & 1.3 & 1.4 & -- \\
\hline
\end{tabular}


Table 19.- Concentration data for dissolved trace elements in water samples collected from the Mississippi River and some of its tributaries during the November-December 1987 cruise Continued

\begin{tabular}{|c|c|c|c|c|c|c|}
\hline \multirow[b]{2}{*}{ Sampling site } & \multicolumn{3}{|c|}{ Vanadium } & \multicolumn{3}{|c|}{ Zinc } \\
\hline & A & B & Grab & A & B & Grab \\
\hline 11-29 Illinois River below Meredosia, Ill. & 0.11 & - & - & 4.9 & - & -- \\
\hline 11-30 Mississippi River near Winfield, Mo. & 0.32 & 0.27 & - & 1.2 & 0.71 & - \\
\hline 12-02 Missouri River at St. Charles, Mo. & 1.6 & 1.4 & - & 2.9 & 2.7 & - \\
\hline 12-03 Mississippi River at St. Louis, Mo. & 1.2 & 1.2 & - & 5.6 & 1.3 & - \\
\hline 12-05 Mississippi River at Thebes, Ill. & 1.1 & 1 & - & 5.3 & 2.2 & - \\
\hline 12-06 Ohio River at Olmsted, Ill. & $<0.07$ & $<0.07$ & - & -- & 4.3 & -- \\
\hline 12-07 Mississippi River below Hickman, Ky. & 0.45 & 0.59 & - & 1.9 & 1.9 & - \\
\hline 12-08 Mississippi River at Fulton, Tenn. & 0.76 & 0.8 & -- & 3.7 & 1.9 & - \\
\hline 12-11 Mississippi River at Helena, Ark. & $<0.07$ & $<0.07$ & -- & 3 & 2.2 & - \\
\hline 12-12 White River at Mile 11.5, Ark. & 0.13 & $<0.07$ & -- & 3.9 & 2.4 & - \\
\hline 12-13 Mississippi River above Arkansas City, Ark. & 0.85 & 0.97 & -- & 1.9 & 1.5 & - \\
\hline 12-14 Yazoo River at Mile 10, Miss. & $<0.07$ & - & - & 2.9 & - & - \\
\hline 12-15 Mississippi River below Vicksburg, Miss. & 0.39 & 0.56 & - & 1.1 & 1.9 & - \\
\hline $\begin{array}{l}\text { 12-17 Old River Outflow Channel near Knox } \\
\text { Landing, La. }\end{array}$ & 0.47 & 0.38 & - & 4.9 & 2.3 & - \\
\hline 12-18 Mississippi River near St. Francisville, La. & 0.41 & 0.41 & -- & 1 & 3.1 & - \\
\hline 12-20 Mississippi River below Belle Chasse, La. & 0.1 & 0.47 & - & 2.1 & 1.3 & - \\
\hline
\end{tabular}

\section{Table 20. - Concentration data for dissolved trace elements in water samples collected from the Mississippi River and some of its tributaries during the May-June 1988 cruise}

[ A, Composite A; B, Composite B; -, not determined; <, less than the detection limit; All results are in micrograms per liter, except for iron and strontium, which are in milligrams per liter ]

\begin{tabular}{|c|c|c|c|c|c|c|c|c|c|c|}
\hline \multirow{2}{*}{$\begin{array}{l}\text { Date } \\
1988\end{array}$} & \multirow[b]{2}{*}{ Sampling site } & \multicolumn{3}{|c|}{ Aluminum } & \multicolumn{3}{|c|}{ Arsenic } & \multicolumn{3}{|c|}{ Barium } \\
\hline & & A & B & Grab & A & B & Grab & A & B & Grab \\
\hline $5-16$ & Illinois River below Meredosia, Ill. & 6.7 & 9.3 & 4.7 & 2.2 & 2.3 & 2.4 & 53 & 55 & 54 \\
\hline $5-17$ & Mississippi River near Winfield, Mo. & 5.6 & 5.4 & 3.4 & 1.6 & 1.7 & 1.5 & 49 & 52 & 51 \\
\hline $5-19$ & Missouri River at Hermann, Mo. & 2.7 & 3.4 & 4.8 & 2.9 & 2.8 & 3.1 & 94 & 94 & 93 \\
\hline $5-20$ & Mississippi River at St. Louis, Mo. & - & 3.9 & 4.1 & 2.3 & 2.3 & 2.8 & 77 & 75 & 59 \\
\hline $5-22$ & Mississippi River at Thebes, Ill. & $<0.2$ & 0.39 & 0.8 & 1.2 & 1.4 & 1.7 & 72 & 74 & 76 \\
\hline $5-23$ & Ohio River at Olmsted, Ill. & 6.2 & 7 & 7 & 0.63 & 0.81 & 1.1 & 38 & 39 & 40 \\
\hline $5-24$ & Mississippi River below Hickman, Ky. & 3.8 & 4.2 & 2.4 & 1.4 & 1.4 & 1.8 & 62 & 65 & 75 \\
\hline $5-26$ & Mississippi River at Fulton, Tenn. & 2.8 & 3.3 & 3.1 & 1.5 & 1.5 & 1.5 & 63 & 63 & 62 \\
\hline $5-28$ & Mississippi River at Helena, Ark. & 130 & - & 240 & 1.7 & 1.6 & 1.6 & 69 & 63 & 70 \\
\hline $5-29$ & White River at Mile 11.5, Ark. & - & 3.1 & 3.2 & 0.95 & 0.86 & 0.93 & 38 & 38 & 38 \\
\hline $5-29$ & Arkansas River at Mile 1.0, Ark. & -- & -- & 660 & - & - & 8.6 & - & - & 400 \\
\hline $5-30$ & Mississippi River above Arkansas City, Ark. & 17 & 14 & 4.1 & 1.4 & 1.4 & 1.2 & 64 & 63 & 57 \\
\hline 6-01 & Yazoo River at Mile 10, Miss. & 6.3 & - & 7.6 & 1.3 & _- & 1.5 & 76 & - & 78 \\
\hline 6-02 & Mississippi River below Vicksburg, Miss. & 5.4 & 6.6 & 7.8 & 1.2 & 1.3 & 1.3 & 59 & 61 & 63 \\
\hline $6-04$ & $\begin{array}{l}\text { Old River Outflow Channel near Knox } \\
\text { Landing, La. }\end{array}$ & 0.95 & 5 & $<0.2$ & 0.89 & 0.8 & $<0.6$ & 62 & 62 & $<0.1$ \\
\hline 6-05 & Mississippi River near St. Francisville, La. & 1.5 & 1.5 & 3.5 & 0.92 & 0.97 & 0.89 & 66 & 67 & 69 \\
\hline 6-07 & Mississippi River below Belle Chasse, La. & - & 14 & 9.9 & 1.1 & 1.1 & 1.2 & 71 & 71 & 70 \\
\hline
\end{tabular}


Table 20.- Concentration data for dissolved trace elements in water samples collected from the Mississippi River and some of its tributaries during the May-June 1988 cruise - Continued

\begin{tabular}{|c|c|c|c|c|c|c|c|c|c|c|}
\hline \multirow{2}{*}{$\begin{array}{l}\text { Date } \\
1988\end{array}$} & \multirow[b]{2}{*}{ Sampling site } & \multicolumn{3}{|c|}{ Beryllium } & \multicolumn{3}{|c|}{ Boron } & \multicolumn{3}{|c|}{ Cadmium } \\
\hline & & A & B & Grab & $\mathbf{A}$ & B & Grab & A & B & Grab \\
\hline $5-16$ & Illinois River below Meredosia, Ill. & 0.14 & 0.14 & 0.13 & 120 & 120 & 120 & 0.4 & 0.46 & 0.42 \\
\hline $5-17$ & Mississippi River near Winfield, Mo. & 0.13 & 0.13 & 0.14 & 29 & 28 & 26 & 0.3 & 0.43 & 0.28 \\
\hline $5-19$ & Missouri River at Hermann, Mo. & 0.14 & 0.15 & 0.14 & 95 & 87 & 79 & 0.42 & 0.56 & 0.37 \\
\hline $5-20$ & Mississippi River at St. Louis, Mo. & 0.16 & 0.14 & 0.14 & 59 & 54 & 57 & 0.46 & 0.71 & 0.52 \\
\hline $5-22$ & Mississippi River at Thebes, Ill. & $<0.02$ & $<0.02$ & $<0.02$ & 49 & 53 & 53 & $<0.1$ & $<0.1$ & $<0.1$ \\
\hline $5-23$ & Ohio River at Olmsted, Ill. & 0.062 & 0.058 & 0.063 & 39 & 38 & 40 & $<0.1$ & 0.18 & $<0.1$ \\
\hline $5-24$ & Mississippi River below Hickman, Ky. & 0.064 & 0.06 & 0.061 & 50 & 51 & 54 & 0.1 & $<0.1$ & 0.16 \\
\hline $5-26$ & Mississippi River at Fulton, Tenn. & 0.59 & 0.59 & 0.59 & 49 & 47 & 47 & 0.56 & 0.57 & 0.54 \\
\hline $5-28$ & Mississippi River at Helena, Ark. & 0.61 & 0.6 & 0.61 & 48 & 45 & 45 & 0.72 & 0.56 & 0.55 \\
\hline $5-29$ & White River at Mile 11.5, Ark. & 0.59 & 0.58 & 0.61 & 11 & 11 & 10 & 0.76 & 0.54 & 0.61 \\
\hline $5-29$ & Arkansas River at Mile 1.0, Ark. & - & - & 0.56 & - & - & 170 & - & - & 0.89 \\
\hline $5-30$ & Mississippi River above Arkansas City, Ark. & 0.38 & 0.36 & 0.34 & 48 & 44 & 39 & 0.44 & 0.21 & 0.15 \\
\hline $6-01$ & Yazoo River at Mile 10, Miss. & 0.36 & - & 0.4 & 18 & - & 19 & 0.17 & - & 0.16 \\
\hline $6-02$ & Mississippi River below Vicksburg, Miss. & 0.37 & 0.35 & 0.38 & 42 & 42 & 41 & 0.23 & 0.18 & 0.19 \\
\hline $6-04$ & $\begin{array}{l}\text { Old River Outflow Channel near Knox } \\
\text { Landing, La. }\end{array}$ & $<0.02$ & $<0.02$ & $<0.02$ & 37 & 37 & $<0.4$ & $<0.1$ & $<0.1$ & $<0.1$ \\
\hline $6-05$ & Mississippi River near St. Francisville, La. & $<0.02$ & $<0.02$ & $<0.02$ & 41 & 40 & 37 & $<0.1$ & $<0.1$ & $<0.1$ \\
\hline $6-07$ & Mississippi River below Belle Chasse, La. & $<0.02$ & $<0.02$ & $<0.02$ & 36 & 36 & 34 & $<0.1$ & $<0.1$ & $<0.1$ \\
\hline
\end{tabular}

Table 20.- Concentration data for dissolved trace elements in water samples collected from the Mississippi River and some of its tributaries during the May-June 1988 cruise - Continued

\begin{tabular}{|c|c|c|c|c|c|c|c|c|c|c|}
\hline \multirow{2}{*}{$\begin{array}{l}\text { Date } \\
1988\end{array}$} & \multirow[b]{2}{*}{ Sampling site } & \multicolumn{3}{|c|}{ Chromium } & \multicolumn{3}{|c|}{ Cobalt } & \multicolumn{3}{|c|}{ Copper } \\
\hline & & $\bar{A}$ & B & Grab & A & B & Grab & A & B & Grab \\
\hline $5-16$ & Illinois River below Meredosia, Ill. & 0.34 & 0.57 & 0.7 & 0.52 & 0.62 & 0.55 & 2.3 & 2.4 & 6.3 \\
\hline $5-17$ & Mississippi River near Winfield, Mo. & 0.54 & 0.6 & 0.78 & 0.36 & 0.2 & 0.32 & 1.2 & 1.4 & 1.4 \\
\hline $5-19$ & Missouri River at Hermann, Mo. & 0.52 & 0.63 & 0.5 & $<0.008$ & $<0.008$ & 0.088 & 1.9 & 1.9 & 1.9 \\
\hline $5-20$ & Mississippi River at St. Louis, Mo. & 1.2 & 0.96 & 1.3 & 0.34 & 0.19 & 0.42 & 1.6 & 1.6 & 1.7 \\
\hline $5-22$ & Mississippi River at Thebes, Ill. & $<0.2$ & $<0.2$ & $<0.2$ & $<0.008$ & $<0.008$ & $<0.008$ & 0.066 & 0.12 & 0.22 \\
\hline $5-23$ & Ohio River at Olmsted, Ill. & 0.33 & $<0.2$ & $<0.2$ & $<0.008$ & 0.071 & 0.06 & 0.96 & 1.2 & 1.2 \\
\hline $5-24$ & Mississippi River below Hickman, Ky. & 0.34 & $<0.2$ & 0.47 & $<0.008$ & 0.022 & $<0.008$ & 1.2 & 1.1 & 1.2 \\
\hline $5-26$ & Mississippi River at Fulton, Tenn. & 1 & 0.82 & 1.1 & 0.46 & 0.53 & 0.5 & 1.7 & 2.8 & 1.7 \\
\hline $5-28$ & Mississippi River at Helena, Ark. & 1.4 & 1.1 & 1.2 & 0.62 & 0.46 & 0.79 & 2 & 1.7 & 2.8 \\
\hline $5-29$ & White River at Mile 11.5, Ark. & 1.9 & 1.5 & 0.98 & 0.49 & 0.43 & 0.46 & 0.68 & 0.63 & 0.93 \\
\hline $5-29$ & Arkansas River at Mile 1.0, Ark. & - & - & 7.8 & - & - & 5.1 & - & - & 8.1 \\
\hline $5-30$ & Mississippi River above Arkansas City, Ark. & 1.1 & 0.61 & 0.97 & 0.15 & 0.14 & $<0.008$ & 1.5 & 1.5 & 1.5 \\
\hline $6-01$ & Yazoo River at Mile 10, Miss. & 1.5 & - & 2 & $<0.008$ & - & 0.019 & 1.1 & - & 1.4 \\
\hline $6-02$ & Mississippi River below Vicksburg, Miss. & 1.6 & 1.1 & 1.8 & $<0.008$ & 0.016 & $<0.008$ & 1.4 & 1.5 & 1.7 \\
\hline $6-04$ & $\begin{array}{l}\text { Old River Outflow Channel near Knox } \\
\text { Landing, La. }\end{array}$ & $<0.2$ & $<0.2$ & $<0.2$ & $<0.008$ & $<0.008$ & $<0.008$ & 0.067 & 0.077 & $<0.02$ \\
\hline $6-05$ & Mississippi River near St. Francisville, La. & $<0.2$ & $<0.2$ & $<0.2$ & $<0.008$ & $<0.008$ & $<0.008$ & 0.03 & 0.049 & 0.09 \\
\hline $6-07$ & Mississippi River below Belle Chasse, La. & $<0.2$ & $<0.2$ & $<0.2$ & $<0.008$ & $<0.008$ & $<0.008$ & 0.45 & 0.36 & 0.37 \\
\hline
\end{tabular}


Table 20.- Concentration data for dissolved trace elements in water samples collected from the Mississippi River and some of its tributaries during the May-June 1988 cruise - Continued

\begin{tabular}{|c|c|c|c|c|c|c|c|c|c|c|}
\hline \multirow{2}{*}{$\begin{array}{l}\text { Date } \\
1988\end{array}$} & \multirow[b]{2}{*}{ Sampling site } & \multicolumn{3}{|c|}{ Iron } & \multicolumn{3}{|c|}{ Lead } & \multicolumn{3}{|c|}{ Lithium } \\
\hline & & A & B & Grab & A & $\mathbf{B}$ & Grab & A & $\mathbf{B}$ & Grab \\
\hline $5-16$ & Illinois River below Meredosia, Ill. & $<0.005$ & $<0.005$ & $<0.005$ & 0.27 & 0.32 & 0.26 & 6.3 & 6.7 & 6.5 \\
\hline $5-17$ & Mississippi River near Winfield, Mo. & $<0.005$ & 0.005 & 0.005 & 0.19 & 0.15 & 0.24 & 5.8 & 5.9 & 5.8 \\
\hline $5-19$ & Missouri River at Hermann, Mo. & $<0.005$ & $<0.005$ & $<0.005$ & 0.24 & 0.26 & 0.16 & 36 & 37 & 35 \\
\hline $5-20$ & Mississippi River at St. Louis, Mo. & 0.015 & $<0.005$ & $<0.005$ & 0.35 & 0.37 & 0.42 & 19 & 19 & 11 \\
\hline $5-22$ & Mississippi River at Thebes, III. & $<0.005$ & $<0.005$ & $<0.005$ & $<0.06$ & $<0.06$ & $<0.06$ & 16 & 16 & 17 \\
\hline $5-23$ & Ohio River at Olmsted, Ill. & $<0.005$ & $<0.005$ & $<0.005$ & $<0.06$ & $<0.06$ & 0.078 & 4.7 & - & 5.1 \\
\hline $5-24$ & Mississippi River below Hickman, Ky. & $<0.005$ & $<0.005$ & $<0.005$ & $<0.06$ & $<0.06$ & $<0.06$ & 12 & 13 & 16 \\
\hline $5-26$ & Mississippi River at Fulton, Tenn. & $<0.005$ & $<0.005$ & $<0.005$ & 0.062 & 0.16 & 0.07 & 12 & 12 & 12 \\
\hline $5-28$ & Mississippi River at Helena, Ark. & 0.28 & $<0.005$ & 0.53 & 0.54 & 0.089 & 0.75 & 11 & 11 & 11 \\
\hline $5-29$ & White River at Mile 11.5, Ark. & 0.041 & $<0.005$ & $<0.005$ & 0.12 & 0.094 & 0.1 & 0.91 & 0.89 & 0.91 \\
\hline $5-29$ & Arkansas River at Mile 1.0, Ark. & - & - & 0.24 & - & - & 1.7 & - & - & 19 \\
\hline $5-30$ & Mississippi River above Arkansas City, Ark. & 0.028 & 0.029 & $<0.005$ & 0.43 & 0.4 & 0.33 & 11 & 10 & 9.3 \\
\hline $6-01$ & Yazoo River at Mile 10, Miss. & $<0.005$ & - & 0.005 & 0.38 & - & 0.35 & 2.7 & - & 2.9 \\
\hline 6-02 & Mississippi River below Vicksburg, Miss. & $<0.005$ & $<0.005$ & $<0.005$ & 0.36 & 0.36 & 0.34 & 9.3 & 9.7 & 9.8 \\
\hline $6-04$ & $\begin{array}{l}\text { Old River Outflow Channel near Knox } \\
\text { Landing, La. }\end{array}$ & $<0.005$ & $<0.005$ & $<0.005$ & $<0.06$ & $<0.06$ & $<0.06$ & 9 & 9.2 & $<0.03$ \\
\hline 6-05 & Mississippi River near St. Francis & $<0.005$ & $<0.005$ & $<0.005$ & $<0.06$ & $<0.06$ & $<0.06$ & 8.7 & 8.6 & 9.1 \\
\hline 6-07 & Mississippi River below Belle Chasse, La. & 0.2 & $<0.005$ & $<0.005$ & $<0.06$ & $<0.06$ & $<0.06$ & 9 & 9.3 & 9.1 \\
\hline
\end{tabular}

Table 20.- Concentration data for dissolved trace elements in water samples collected from the Mississippi River and some of its tributaries during the May-June 1988 cruise - Continued

\begin{tabular}{|c|c|c|c|c|c|c|c|c|c|c|}
\hline \multirow{2}{*}{$\begin{array}{l}\text { Date } \\
1988\end{array}$} & \multirow[b]{2}{*}{ Sampling site } & \multicolumn{3}{|c|}{ Manganese } & \multicolumn{3}{|c|}{ Molybdenum } & \multicolumn{3}{|c|}{ Nickel } \\
\hline & & $\bar{A}$ & B & Grab & $\mathbf{A}$ & $\mathbf{B}$ & Grab & $\bar{A}$ & B & Grab \\
\hline $5-16$ & Illinois River below Meredosia, Ill. & 0.74 & - & 0.97 & 5.8 & 5.7 & 5.7 & 6.3 & 10 & 10 \\
\hline $5-17$ & Mississippi River near Winfield, Mo. & 6.5 & 4.9 & 7.3 & 1.2 & 1.3 & 1.3 & 2.7 & 1.1 & 1.1 \\
\hline $5-19$ & Missouri River at Hermann, Mo. & 0.65 & 0.84 & 0.55 & 3 & 3.1 & 3 & $<0.03$ & $<0.03$ & 1.4 \\
\hline $5-20$ & Mississippi River at St. Louis, Mo. & - & 0.9 & 0.81 & 2.9 & 2.6 & 2.6 & 3.1 & 0.85 & 3.7 \\
\hline $5-22$ & Mississippi River at Thebes, III. & $<0.06$ & $<0.06$ & $<0.06$ & 2.2 & 2.4 & 2.5 & 0.34 & 2.8 & 4.9 \\
\hline $5-23$ & Ohio River at Olmsted, Ill. & 0.36 & 0.37 & 0.34 & 2.8 & 2.7 & 2.9 & $<0.03$ & $<0.03$ & $<0.03$ \\
\hline $5-24$ & Mississippi River below Hickman, Ky. & 0.42 & 0.51 & 0.44 & 2.9 & 2.9 & 3 & $<0.03$ & $<0.03$ & $<0.03$ \\
\hline $5-26$ & Mississippi River at Fulton, Tenn. & 1.3 & 1.4 & 1.4 & 2.6 & 2.5 & 2.5 & 2.2 & 3.3 & 3.1 \\
\hline $5-28$ & Mississippi River at Helena, Ark. & 44 & - & 66 & 2.4 & 2.5 & 2.1 & 2.5 & 1.6 & 3 \\
\hline $5-29$ & White River at Mile 11.5, Ark. & 9.1 & 2.6 & 2.2 & 0.9 & 0.95 & 0.92 & 0.88 & 0.96 & 1.1 \\
\hline $5-29$ & Arkansas River at Mile 1.0, Ark. & - & - & 180 & - & -- & 4 & -- & - & 99 \\
\hline $5-30$ & Mississippi River above Arkansas City, Ark. & 3.9 & 3.1 & 0.45 & 2.7 & 2.6 & 2.4 & 4.5 & 4 & 1.1 \\
\hline $6-01$ & Yazoo River at Mile 10 , Miss. & 100 & - & 100 & 1.3 & - & 1.4 & $<0.03$ & - & $<0.03$ \\
\hline $6-02$ & Mississippi River below Vicksburg, Miss. & 0.28 & 0.6 & 1.3 & 2.4 & 2.5 & 2.5 & $<0.03$ & 0.97 & 0.99 \\
\hline $6-04$ & $\begin{array}{l}\text { Old River Outflow Channel near Knox } \\
\text { Landing, La. }\end{array}$ & $<0.06$ & $<0.06$ & $<0.06$ & 2.2 & 2.2 & $<0.09$ & 3.9 & 3 & $<0.03$ \\
\hline $6-05$ & Mississippi River near St. Francisville, La. & $<0.06$ & $<0.06$ & 2.1 & 2.3 & 2.2 & 2.3 & 1.7 & 1.6 & 2 \\
\hline $6-07$ & Mississippi River below Belle Chasse, La. & - & 0.24 & $<0.06$ & 2 & 2.2 & 2.2 & 2.1 & 6.1 & 4.7 \\
\hline
\end{tabular}


Table 20.- Concentration data for dissolved trace elements in water samples collected from the Mississippi River and some of its tributaries during the May-June 1988 cruise - Continued

\begin{tabular}{|c|c|c|c|c|c|c|c|c|c|c|}
\hline \multirow{2}{*}{$\begin{array}{l}\text { Date } \\
1988\end{array}$} & \multirow[b]{2}{*}{ Sampling site } & \multicolumn{3}{|c|}{ Strontium } & \multicolumn{3}{|c|}{ Thallium } & \multicolumn{3}{|c|}{ Uranium } \\
\hline & & $\mathbf{A}$ & B & Grab & A & B & Grab & $\bar{A}$ & B & Grab \\
\hline $5-16$ & Illinois River below Meredosia, Ill. & 0.19 & 0.2 & 0.2 & $<0.05$ & $<0.05$ & $<0.05$ & 1.3 & 1.2 & 1.1 \\
\hline $5-17$ & Mississippi River near Winfield, Mo. & 0.094 & 0.11 & 0.11 & $<0.05$ & $<0.05$ & $<0.05$ & 1.3 & 1.4 & 1.4 \\
\hline $5-19$ & Missouri River at Hermann, Mo. & 0.47 & 0.47 & 0.41 & $<0.05$ & $<0.05$ & $<0.05$ & 3.8 & 3.1 & 2.7 \\
\hline $5-20$ & Mississippi River at St. Louis, Mo. & 0.26 & 0.27 & 0.17 & $<0.05$ & $<0.05$ & $<0.05$ & 1.5 & 1.4 & 1.8 \\
\hline $5-22$ & Mississippi River at Thebes, Ill. & 0.28 & 0.27 & 0.26 & $<0.05$ & $<0.05$ & $<0.05$ & 3 & 3 & 3.3 \\
\hline $5-23$ & Ohio River at Olmsted, Ill. & 0.2 & 0.19 & 0.2 & 0.37 & 0.4 & 0.38 & 0.91 & 0.95 & 0.98 \\
\hline $5-24$ & Mississippi River below Hickman, Ky. & 0.24 & 0.24 & 0.26 & 0.37 & 0.37 & 0.36 & 2.3 & 2.3 & 2.8 \\
\hline $5-26$ & Mississippi River at Fulton, Tenn. & 0.23 & 0.22 & 0.22 & $<0.05$ & $<0.05$ & $<0.05$ & 2 & 2.1 & 2.1 \\
\hline $5-28$ & Mississippi River at Helena, Ark. & 0.22 & 0.21 & 0.22 & $<0.05$ & 0.051 & $<0.05$ & 2.1 & 2.1 & 2 \\
\hline $5-29$ & White River at Mile 11.5, Ark. & 0.039 & 0.041 & 0.04 & $<0.05$ & $<0.05$ & $<0.05$ & 0.61 & 0.6 & 0.62 \\
\hline $5-29$ & Arkansas River at Mile 1.0, Ark. & -- & - & 0.36 & - & -- & 0.41 & - & - & 6.4 \\
\hline $5-30$ & Mississippi River above Arkansas City, Ark. & 0.2 & 0.2 & 0.21 & 0.14 & 0.14 & 0.11 & 2 & 2 & 1.8 \\
\hline $6-01$ & Yazoo River at Mile 10, Miss. & 0.12 & - & 0.12 & 0.13 & - & 0.13 & 0.68 & - & 0.72 \\
\hline 6-02 & Mississippi River below Vicksburg, Miss. & 0.22 & 0.22 & 0.22 & 0.13 & 0.14 & 0.13 & 1.9 & 2 & 1.9 \\
\hline 6-04 & $\begin{array}{l}\text { Old River Outflow Channel near Knox } \\
\text { Landing, La. }\end{array}$ & 0.21 & 0.22 & $<0.001$ & $<0.05$ & $<0.05$ & $<0.05$ & 1.6 & 1.6 & $<0.06$ \\
\hline $6-05$ & Mississippi River near St. Fr & 0.22 & 0.22 & 0.22 & $<0.05$ & $<0.05$ & $<0.05$ & 1.5 & 1.6 & 1.7 \\
\hline $6-07$ & Mississippi River below Belle Chasse, La. & 0.23 & 0.22 & 0.22 & $<0.05$ & $<0.05$ & $<0.05$ & 1.7 & 1.8 & 1.7 \\
\hline
\end{tabular}

Table 20.-- Concentration data for dissolved trace elements in water samples collected from the Mississippi River and some of its tributaries during the May-June 1988 cruise - Continued

\begin{tabular}{|c|c|c|c|c|c|c|c|}
\hline \multirow{2}{*}{$\begin{array}{l}\text { Date } \\
1988\end{array}$} & \multirow[b]{2}{*}{ Sampling site } & \multicolumn{3}{|c|}{ Vanadium } & \multicolumn{3}{|c|}{ Zinc } \\
\hline & & A & B & Grab & A & B & Grab \\
\hline $5-16$ & Illinois River below Meredosia, Ill. & 1.2 & 1.6 & 1.2 & 3.4 & 4 & 6.7 \\
\hline $5-17$ & Mississippi River near Winfield, Mo. & 1.2 & 1.2 & 0.97 & 0.99 & 1 & 2.1 \\
\hline $5-19$ & Missouri River at Hermann, Mo. & 2.9 & 2.7 & 4.3 & 1.4 & 1.5 & 1.4 \\
\hline $5-20$ & Mississippi River at St. Louis, Mo. & 1.9 & 1.7 & 4.3 & 1 & 1.3 & 1 \\
\hline $5-22$ & Mississippi River at Thebes, Ill. & 1.1 & 1.5 & 4.1 & $<0.08$ & $<0.08$ & 0.083 \\
\hline $5-23$ & Ohio River at Olmsted, Ill. & $<0.07$ & $<0.07$ & 2.2 & 0.43 & - & 0.69 \\
\hline $5-24$ & Mississippi River below Hickman, Ky. & $<0.07$ & $<0.07$ & 0.099 & 0.59 & $<0.08$ & $<0.08$ \\
\hline $5-26$ & Mississippi River at Fulton, Tenn. & $<0.07$ & $<0.07$ & $<0.07$ & 0.96 & 2.1 & 1.3 \\
\hline $5-28$ & Mississippi River at Helena, Ark. & $<0.07$ & $<0.07$ & $<0.07$ & 3.5 & 1.9 & 5.5 \\
\hline $5-29$ & White River at Mile 11.5, Ark. & $<0.07$ & $<0.07$ & $<0.07$ & 1.7 & 1.2 & 1.2 \\
\hline $5-29$ & Arkansas River at Mile 1.0, Ark. & - & - & 12 & - & - & 17 \\
\hline $5-30$ & Mississippi River above Arkansas City, Ark. & $<0.07$ & $<0.07$ & $<0.07$ & 1.1 & 1.6 & 0.6 \\
\hline $6-01$ & Yazoo River at Mile 10, Miss. & $<0.07$ & - & $<0.07$ & 1.2 & - & 0.98 \\
\hline $6-02$ & Mississippi River below Vicksburg, Miss. & $<0.07$ & $<0.07$ & $<0.07$ & 1.5 & 1.9 & 0.76 \\
\hline $6-04$ & $\begin{array}{l}\text { Old River Outflow Channel near Knox } \\
\text { Landing, La. }\end{array}$ & 0.57 & 0.69 & $<0.07$ & $<0.08$ & $<0.08$ & $<0.08$ \\
\hline $6-05$ & Mississippi River near St. Francisville, La. & 1.6 & 1.6 & 1.1 & $<0.08$ & $<0.08$ & $<0.08$ \\
\hline $6-07$ & Mississippi River below Belle Chasse, La. & 0.89 & 0.77 & 4.2 & $<0.08$ & $<0.08$ & $<0.08$ \\
\hline
\end{tabular}




\section{Table 21.- Concentration data for dissolved trace elements in water samples collected from the Mississippi River and some of its tributaries during the March-April 1989 cruise}

[ A, Composite A; B, Composite B; -, not determined; <, less than the detection limit; All results are in micrograms per liter, except for iron and strontium, which are in milligrams per liter ]

\begin{tabular}{|c|c|c|c|c|c|c|c|c|c|c|}
\hline \multirow{2}{*}{$\begin{array}{l}\text { Date } \\
1989\end{array}$} & \multirow[b]{2}{*}{ Sampllng site } & \multicolumn{3}{|c|}{ Alumlnum } & \multicolumn{3}{|c|}{ Arsenic } & \multicolumn{3}{|c|}{ Barlum } \\
\hline & & A & $\mathbf{B}$ & Grab & A & B & Grab & A & B & Grab \\
\hline $3-09$ & Illinois River at Hardin, Ill. & 5.5 & 3.1 & 2.4 & 2.3 & 1.7 & 2.2 & 50 & 51 & 50 \\
\hline $3-10$ & Mississippi River near Winfield, Mo. & 3.2 & 3.9 & 5.2 & 0.99 & 0.91 & 0.93 & 45 & 46 & 46 \\
\hline $3-12$ & Missouri River at Hermann, Mo. & 8 & 9.7 & 5 & 1.8 & 1.6 & 1.7 & 66 & 65 & 61 \\
\hline $3-13$ & Mississippi River at St. Louis, Mo. & 3.1 & 4.2 & - & 1.6 & 1.5 & - & 54 & 54 & - \\
\hline $3-15$ & Mississippi River at Thebes, Ill. & 3 & 2.3 & 3 & 0.78 & 1.3 & 1.8 & 59 & 59 & 58 \\
\hline $3-16$ & Ohio River at Olmsted, Ill. & 9.8 & 7.2 & 7.6 & $<0.6$ & $<0.6$ & $<0.6$ & 27 & 27 & 26 \\
\hline $3-17$ & Mississippi River below Hickman, Ky. & 10 & 11 & 15 & $<0.6$ & $<0.6$ & $<0.6$ & 34 & 34 & 33 \\
\hline $3-19$ & Mississippi River below Fulton, Tenn. & 5.9 & 6.2 & 6.7 & $<0.6$ & $<0.6$ & $<0.6$ & 36 & 36 & 37 \\
\hline 3-21 & Mississippi River at Helena, Ark. & 100 & 230 & 230 & 0.68 & $<0.6$ & 0.78 & 42 & 42 & 46 \\
\hline $3-22$ & White River at Mile 11.5, Ark. & 9.8 & 7.5 & 2.9 & 1.1 & 0.87 & $<0.6$ & 31 & 31 & 31 \\
\hline $3-23$ & Arkansas River at Pendleton, & 5.4 & 7 & 4.1 & 1.1 & 0.81 & 0.9 & 49 & 50 & 52 \\
\hline 3-24 & Mississippi River above Arkansas City, Ark. & 4 & 5.1 & 3.9 & 0.72 & 1.2 & 0.91 & 44 & 43 & 43 \\
\hline $3-26$ & Yazoo River below Steele Bayou, Miss. & - & 23 & 35 & 1.9 & 2.1 & 2.2 & 25 & 25 & 27 \\
\hline 3-27 & Mississippi River below Vicksburg, Miss. & 4.1 & 3.9 & 4.5 & 1 & 0.9 & 0.9 & 45 & 46 & 46 \\
\hline 3-29 & $\begin{array}{l}\text { Old River Outflow Channel near Knox } \\
\text { Landing, La. }\end{array}$ & 2.6 & 5.3 & 1.9 & 1.5 & 1.3 & 0.77 & 47 & 47 & 48 \\
\hline 3-30 & Mississippi River & 2.3 & 2.1 & 2.1 & 1.5 & 0.78 & 0.98 & 48 & 47 & 48 \\
\hline 4-01 & Mississippi River below Belle Chasse, & 3.7 & 2.8 & 3.2 & 1.2 & 0.77 & 0.67 & 49 & 49 & 50 \\
\hline
\end{tabular}

Table 21.- Concentration data for dissolved trace elements in water samples collected from the Mississippi River and some of its tributaries during the March-April 1989 cruise - Continued

\begin{tabular}{|c|c|c|c|c|c|c|c|c|c|c|}
\hline \multirow{2}{*}{$\begin{array}{l}\text { Date } \\
1989\end{array}$} & \multirow[b]{2}{*}{ Sampling site } & \multicolumn{3}{|c|}{ Beryllium } & \multicolumn{3}{|c|}{ Boron } & \multicolumn{3}{|c|}{ Cadmium } \\
\hline & & A & B & Grab & A & B & Grab & A & B & Grab \\
\hline 3-09 & Illinois River at Hardin, Ill. & $<0.02$ & $<0.02$ & $<0.02$ & 130 & 140 & 130 & $<0.1$ & $<0.1$ & $<0.1$ \\
\hline $3-10$ & Mississippi River near Winfield, Mo. & $<0.02$ & $<0.02$ & $<0.02$ & 37 & 38 & 38 & $<0.1$ & $<0.1$ & $<0.1$ \\
\hline $3-12$ & Missouri River at Hermann, Mo. & $<0.02$ & $<0.02$ & $<0.02$ & 54 & 48 & 44 & $<0.1$ & $<0.1$ & $<0.1$ \\
\hline $3-13$ & Mississippi River at St. Louis, Mo. & $<0.02$ & $<0.02$ & - & 61 & 60 & - & $<0.1$ & $<0.1$ & - \\
\hline 3-15 & Mississippi River at Thebes, Ill. & $<0.02$ & $<0.02$ & $<0.02$ & 57 & 55 & 56 & $<0.1$ & $<0.1$ & $<0.1$ \\
\hline $3-16$ & Ohio River at Olmsted, Ill. & $<0.02$ & $<0.02$ & $<0.02$ & 15 & 17 & 15 & $<0.1$ & $<0.1$ & $<0.1$ \\
\hline $3-17$ & Mississippi River below Hickman, Ky. & $<0.02$ & $<0.02$ & $<0.02$ & 22 & 23 & 21 & $<0.1$ & $<0.1$ & $<0.1$ \\
\hline $3-19$ & Mississippi River below Fulton, Tenn. & $<0.02$ & $<0.02$ & $<0.02$ & 24 & 25 & 25 & $<0.1$ & $<0.1$ & $<0.1$ \\
\hline $3-21$ & Mississippi River at Helena, Ark. & $<0.02$ & $<0.02$ & $<0.02$ & 23 & 25 & 24 & $<0.1$ & $<0.1$ & $<0.1$ \\
\hline $3-22$ & White River at Mile 11.5, Ark. & $<0.02$ & $<0.02$ & 0.022 & 8.1 & 6.8 & 6.2 & $<0.1$ & $<0.1$ & $<0.1$ \\
\hline $3-23$ & Arkansas River at Pendleton, Ark. & $<0.02$ & $<0.02$ & $<0.02$ & 27 & 28 & 30 & $<0.1$ & $<0.1$ & $<0.1$ \\
\hline $3-24$ & Mississippi River above Arkansas City, Ark. & 0.039 & $<0.02$ & $<0.02$ & 24 & 26 & 27 & $<0.1$ & $<0.1$ & $<0.1$ \\
\hline $3-26$ & Yazoo River below Steele Bayou, Miss. & 0.022 & $<0.02$ & $<0.02$ & 13 & 12 & 13 & $<0.1$ & $<0.1$ & $<0.1$ \\
\hline 3-27 & Mississippi River below Vicksburg, Miss. & $<0.02$ & $<0.02$ & $<0.02$ & 23 & 25 & 25 & $<0.1$ & $<0.1$ & $<0.1$ \\
\hline $3-29$ & $\begin{array}{l}\text { Old River Outflow Channel near Knox } \\
\text { Landing, La. }\end{array}$ & $<0.02$ & $<0.02$ & $<0.02$ & 23 & 24 & 23 & $<0.1$ & $<0.1$ & $<0.1$ \\
\hline 3-30 & Mississippi River near St. Francisville, La. & $<0.02$ & $<0.02$ & $<0.02$ & 23 & 23 & 24 & $<0.1$ & $<0.1$ & $<0.1$ \\
\hline 4-01 & Mississippi River below Belle Chasse, La. & $<0.02$ & $<0.02$ & $<0.02$ & 22 & 24 & 23 & $<0.1$ & $<0.1$ & $<0.1$ \\
\hline
\end{tabular}


Table 21. - Concentration data for dissolved trace elements in water samples collected from the Mississippi River and some of its tributaries during the March-April 1989 cruise - Continued

\begin{tabular}{|c|c|c|c|c|c|c|c|c|c|c|}
\hline \multirow{2}{*}{$\begin{array}{l}\text { Date } \\
1989\end{array}$} & \multirow[b]{2}{*}{ Sampling site } & \multicolumn{3}{|c|}{ Chromium } & \multicolumn{3}{|c|}{ Cobalt } & \multicolumn{3}{|c|}{ Copper } \\
\hline & & A & B & Grab & A & B & Grab & A & B & Grab \\
\hline 3-09 & Illinois River at Hardin, Ill. & 0.96 & 0.96 & 0.81 & 0.87 & 0.83 & 0.77 & 2.1 & 2.2 & 2.2 \\
\hline 3-10 & Mississippi River near Winfield, Mo. & 0.67 & 0.77 & 0.54 & 0.44 & 0.48 & 0.44 & 1.2 & 1.2 & 1 \\
\hline $3-12$ & Missouri River at Hermann, Mo. & 0.48 & 0.27 & $<0.2$ & 0.34 & 0.35 & 0.32 & 1 & 1 & 0.88 \\
\hline 3-13 & Mississippi River at St. Louis, Mo. & 0.27 & 0.24 & - & 0.45 & 0.45 & - & 1.3 & 1.2 & - \\
\hline $3-15$ & Mississippi River at Thebes, Ill. & 0.37 & $<0.2$ & 0.21 & 0.44 & 0.41 & 0.41 & 1.2 & 1.4 & 1.1 \\
\hline $3-16$ & Ohio River at Olmsted, Ill. & $<0.2$ & $<0.2$ & $<0.2$ & 0.21 & 0.2 & 0.19 & 0.52 & 0.81 & 0.94 \\
\hline 3-17 & Mississippi River below Hickman, Ky. & $<0.2$ & $<0.2$ & $<0.2$ & 0.21 & 0.25 & 0.24 & 0.65 & 0.82 & 0.73 \\
\hline $3-19$ & Mississippi River below Fulton, Tenn. & $<0.2$ & $<0.2$ & $<0.2$ & 0.23 & 0.23 & 0.24 & 0.74 & 0.73 & 0.72 \\
\hline $3-21$ & Mississippi River at Helena, Ark. & $<0.2$ & $<0.2$ & $<0.2$ & 0.32 & 0.34 & 0.31 & 0.88 & 0.86 & 0.89 \\
\hline 3-22 & White River at Mile 11.5, Ark. & $<0.2$ & $<0.2$ & $<0.2$ & 0.1 & 0.1 & 0.088 & 0.31 & 0.32 & 0.3 \\
\hline $3-23$ & Arkansas River at Pendleton, Ark. & $<0.2$ & $<0.2$ & $<0.2$ & 0.18 & 0.14 & 0.21 & 0.45 & 0.55 & 0.66 \\
\hline 3-24 & Mississippi River above Arkansas City, Ark. & $<0.2$ & $<0.2$ & $<0.2$ & 0.21 & 0.22 & 0.2 & 0.46 & 0.52 & 0.51 \\
\hline $3-26$ & Yazoo River below Steele Bayou, Miss. & $<0.2$ & $<0.2$ & $<0.2$ & 0.03 & 0.031 & 0.042 & 0.69 & 0.7 & 0.81 \\
\hline 3-27 & Mississippi River below Vicksburg, Miss. & $<0.2$ & $<0.2$ & $<0.2$ & 0.19 & 0.19 & 0.19 & 0.49 & 0.48 & 0.48 \\
\hline 3-29 & $\begin{array}{l}\text { Old River Outflow Channel near Knox } \\
\text { Landing, La. }\end{array}$ & $<0.2$ & $<0.2$ & $<0.2$ & 0.19 & 0.15 & 0.16 & 0.92 & 0.94 & 0.91 \\
\hline 3-30 & Mississippi River near St. Francisville, La. & $<0.2$ & $<0.2$ & $<0.2$ & 0.16 & 0.17 & 0.14 & 0.91 & 0.84 & 1.1 \\
\hline 4-01 & Mississippi River below Belle Chasse, La. & $<0.2$ & $<0.2$ & $<0.2$ & 0.13 & 0.13 & 0.13 & 0.93 & 0.91 & 0.9 \\
\hline
\end{tabular}

Table 21. - Concentration data for dissolved trace elements in water samples collected from the Mississippi River and some of its tributaries during the March-April 1989 cruise - Continued

\begin{tabular}{|c|c|c|c|c|c|c|c|c|c|c|}
\hline \multirow{2}{*}{$\begin{array}{l}\text { Date } \\
1989\end{array}$} & \multirow[b]{2}{*}{ Sampling site } & \multicolumn{3}{|c|}{ Iron } & \multicolumn{3}{|c|}{ Lead } & \multicolumn{3}{|c|}{ Llthlum } \\
\hline & & A & B & Grab & A & $\mathbf{B}$ & Grab & A & B & Grab \\
\hline $3-09$ & Illinois River at Hardin, Ill. & $<0.005$ & $<0.005$ & $<0.005$ & 0.27 & 0.24 & 0.29 & 6.2 & 6.7 & 6.4 \\
\hline $3-10$ & Mississippi River near Winfield, Mo. & 0.016 & 0.017 & 0.016 & 0.13 & 0.11 & 0.072 & 3.2 & 3.4 & 3.1 \\
\hline $3-12$ & Missouri River at Hermann, Mo. & 0.006 & 0.011 & $<0.005$ & 0.065 & 0.16 & 0.089 & 16 & 14 & 13 \\
\hline 3-13 & Mississippi River at St. Louis, Mo. & $<0.005$ & 0.005 & - & 0.069 & 0.069 & - & 8.1 & 8.1 & - \\
\hline 3-15 & Mississippi River at Thebes, Ill. & 0.005 & $<0.005$ & $<0.005$ & 0.13 & 0.15 & 0.11 & 8 & 7.8 & 8.1 \\
\hline 3-16 & Ohio River at Olmsted, Ill. & 0.008 & 0.005 & 0.006 & $<0.06$ & $<0.06$ & $<0.06$ & 1.6 & 1.7 & 1.7 \\
\hline $3-17$ & Mississippi River below Hickman, Ky. & 0.006 & $<0.005$ & 0.006 & $<0.06$ & $<0.06$ & $<0.06$ & 2.6 & 2.7 & 2.5 \\
\hline $3-19$ & Mississippi River below Fulton, Tenn. & $<0.005$ & $<0.005$ & $<0.005$ & $<0.06$ & $<0.06$ & $<0.06$ & 2.6 & 2.8 & 2.9 \\
\hline $3-21$ & Mississippi River at Helena, Ark. & 0.095 & 0.17 & 0.16 & $<0.06$ & 0.087 & $<0.06$ & 2.6 & 2.8 & 2.6 \\
\hline $3-22$ & White River at Mile 11.5, Ark. & 0.013 & 0.015 & 0.012 & $<0.06$ & $<0.06$ & $<0.06$ & 0.24 & 0.2 & 0.19 \\
\hline $3-23$ & Arkansas River at Pendleton, Ark. & 0.009 & 0.009 & 0.009 & $<0.06$ & $<0.06$ & $<0.06$ & 2.1 & 2.3 & 2.4 \\
\hline 3-24 & Mississippi River above Arkansas City, Ark. & $<0.005$ & $<0.005$ & $<0.005$ & $<0.06$ & 0.094 & $<0.06$ & 2.9 & 3 & 2.8 \\
\hline $3-26$ & Yazoo River below Steele Bayou, Miss. & 0.028 & 0.036 & 0.047 & $<0.06$ & $<0.06$ & $<0.06$ & 0.86 & 0.83 & 1.1 \\
\hline 3-27 & Mississippi River below Vicksburg, Miss. & $<0.005$ & $<0.005$ & $<0.005$ & $<0.06$ & $<0.06$ & $<0.06$ & 2.9 & 3 & 3.3 \\
\hline $3-29$ & $\begin{array}{l}\text { Old River Outflow Channel near Knox } \\
\text { Landing, La. }\end{array}$ & 0.005 & $<0.005$ & $<0.005$ & $<0.06$ & 0.25 & 0.091 & 3 & 3 & 3.1 \\
\hline 3-30 & Mississippi River near St. Francisville, La. & $<0.005$ & 0.005 & $<0.005$ & $<0.06$ & $<0.06$ & $<0.06$ & 3.2 & 3 & 3.2 \\
\hline 4-01 & Mississippi River below Belle Chasse, La. & 0.005 & $<0.005$ & $<0.005$ & $<0.06$ & $<0.06$ & $<0.06$ & 3 & 3.1 & 3.2 \\
\hline
\end{tabular}


Table 21.- Concentration data for dissolved trace elements in water samples collected from the Mississippi River and some of its tributaries during the March-April 1989 cruise - Continued

\begin{tabular}{|c|c|c|c|c|c|c|c|c|c|c|}
\hline \multirow{2}{*}{$\begin{array}{l}\text { Date } \\
1989\end{array}$} & \multirow[b]{2}{*}{ Sampling slte } & \multicolumn{3}{|c|}{ Manganese } & \multicolumn{3}{|c|}{ Molybdenum } & \multicolumn{3}{|c|}{ Nickel } \\
\hline & & A & B & Grab & A & B & Grab & A & B & Grab \\
\hline 3-09 & Illinois River at Hardin, Ill. & 16 & 18 & 23 & 6.3 & 6.4 & 5.6 & 19 & 19 & 18 \\
\hline $3-10$ & Mississippi River near Winfield, Mo. & 1.7 & 1.9 & 1.4 & 1.3 & 1.3 & 1.4 & 9 & 9.5 & 8.9 \\
\hline $3-12$ & Missouri River at Hermann, Mo. & 4.4 & - & 7 & 1.8 & 1.7 & 1.6 & 7.9 & 7.1 & 6.5 \\
\hline $3-13$ & Mississippi River at St. Louis, Mo. & 2.5 & 3.3 & - & 2.5 & 2.3 & - & 9.3 & 9.1 & - \\
\hline $3-15$ & Mississippi River at Thebes, Ill. & 1.1 & 1.5 & 1.3 & 2.2 & 2.1 & 2.1 & 8.2 & 8.4 & 8.5 \\
\hline $3-16$ & Ohio River at Olmsted, Ill. & 4 & 4 & 4.6 & 0.77 & 0.76 & 0.67 & 4.6 & 4.8 & 4.5 \\
\hline $3-17$ & Mississippi River below Hickman, Ky. & 1.9 & 1.5 & 14 & 0.97 & 1.1 & 0.92 & 5.5 & 5.3 & 5 \\
\hline $3-19$ & Mississippi River below Fulton, Tenn. & 1.6 & 1.7 & 1.9 & 0.94 & 0.93 & 0.99 & 5.3 & 5.6 & 5.8 \\
\hline $3-21$ & Mississippi River at Helena, Ark. & 12 & 10 & 8.3 & 0.93 & 0.88 & 0.83 & 5.5 & 5.8 & 5.5 \\
\hline $3-22$ & White River at Mile 11.5, Ark. & 3.3 & 3.2 & 4.1 & 0.34 & 0.27 & 0.34 & 3.1 & 3.1 & 3 \\
\hline $3-23$ & Arkansas River at Pendleton, Ark. & 1.8 & 2 & 1.5 & 0.55 & 0.59 & 0.58 & 4.3 & 4.1 & 4.6 \\
\hline $3-24$ & Mississippi River above Arkansas City, Ark. & 1.6 & 1.5 & 1.3 & 0.92 & 1 & 1 & 5.3 & 5.4 & 5.1 \\
\hline 3-26 & Yazoo River below Steele Bayou, Miss. & 9.4 & 9.4 & 11 & 0.16 & 0.14 & 0.27 & 2.4 & 2.3 & 2.4 \\
\hline 3-27 & Mississippi River below Vicksburg, Miss. & 0.77 & 0.72 & 0.89 & 0.98 & 0.9 & 0.99 & 5 & 5.2 & 5.2 \\
\hline $3-29$ & $\begin{array}{l}\text { Old River Outflow Channel near Knox } \\
\text { Landing, La. }\end{array}$ & 2.6 & 2.5 & 2.7 & 1 & 0.99 & 1 & 5.4 & 5.3 & 5.6 \\
\hline 3-30 & Mississippi River near St. Francisville, La. & 1.7 & 1.6 & 1.6 & 0.97 & 0.99 & 1 & 5.4 & 5.4 & 5.3 \\
\hline 4-01 & Mississippi River below Belle Chasse, La. & 2.2 & 2.1 & 6.5 & 0.91 & 1.2 & 1 & 5 & 5.1 & 5.1 \\
\hline
\end{tabular}

Table 21.- Concentration data for dissolved trace elements in water samples collected from the Mississippi River and some of its tributaries during the March-April 1989 cruise - Continued

\begin{tabular}{|c|c|c|c|c|c|c|c|c|c|c|}
\hline \multirow{2}{*}{$\begin{array}{l}\text { Date } \\
1989\end{array}$} & \multirow[b]{2}{*}{ Sampiling site } & \multicolumn{3}{|c|}{ Strontlum } & \multicolumn{3}{|c|}{ Thailium } & \multicolumn{3}{|c|}{ Uranium } \\
\hline & & $\overline{\mathbf{A}}$ & B & Grab & $\overline{\mathbf{A}}$ & B & Grab & $\overline{\mathbf{A}}$ & $\mathbf{B}$ & Grab \\
\hline 3-09 & Illinois River at Hardin, Ill. & 0.28 & 0.28 & 0.27 & $<0.05$ & $<0.05$ & $<0.05$ & 1.6 & 1.7 & 1.6 \\
\hline 3-10 & Mississippi River near Winfield, Mo. & 0.094 & 0.093 & 0.092 & $<0.05$ & $<0.05$ & $<0.05$ & 0.78 & 0.8 & 0.74 \\
\hline $3-12$ & Missouri River at Hermann, Mo. & 0.27 & 0.26 & 0.24 & $<0.05$ & $<0.05$ & $<0.05$ & 3.2 & 3.1 & 2.9 \\
\hline 3-13 & Mississippi River at St. Louis, Mo. & 0.19 & 0.2 & -- & $<0.05$ & $<0.05$ & - & 2 & 1.9 & - \\
\hline $3-15$ & Mississippi River at Thebes, Ill. & 0.19 & 0.19 & 0.19 & $<0.05$ & $<0.05$ & $<0.05$ & 1.7 & 1.8 & 1.8 \\
\hline 3-16 & Ohio River at Olmsted, Ill. & 0.11 & 0.11 & 0.11 & $<0.05$ & $<0.05$ & $<0.05$ & 0.21 & 0.14 & 0.13 \\
\hline 3-17 & Mississippi River below Hickman, Ky. & 0.12 & 0.12 & 0.12 & $<0.05$ & $<0.05$ & $<0.05$ & 0.42 & 0.48 & 0.38 \\
\hline 3-19 & Mississippi River below Fulton, Tenn. & 0.12 & 0.12 & 0.12 & $<0.05$ & $<0.05$ & $<0.05$ & 0.41 & 0.53 & 0.44 \\
\hline $3-21$ & Mississippi River at Helena, Ark. & 0.12 & 0.12 & 0.12 & $<0.05$ & $<0.05$ & $<0.05$ & 0.52 & 0.49 & 0.49 \\
\hline $3-22$ & White River at Mile 11.5, Ark. & 0.027 & 0.027 & 0.032 & $<0.05$ & $<0.05$ & $<0.05$ & $<0.06$ & $<0.06$ & $<0.06$ \\
\hline $3-23$ & Arkansas River at Pendleton, Ark. & 0.17 & 0.17 & 0.18 & $<0.05$ & $<0.05$ & $<0.05$ & 0.47 & 0.49 & 0.44 \\
\hline 3-24 & Mississippi River above Arkansas City, Ark. & 0.12 & 0.12 & 0.12 & $<0.05$ & $<0.05$ & $<0.05$ & 0.52 & 0.45 & 0.5 \\
\hline $3-26$ & Yazoo River below Steele Bayou, Miss. & 0.036 & 0.036 & 0.037 & $<0.05$ & $<0.05$ & $<0.05$ & $<0.06$ & $<0.06$ & $<0.06$ \\
\hline 3-27 & Mississippi River below Vicksburg, Miss. & 0.12 & 0.12 & 0.13 & $<0.05$ & $<0.05$ & $<0.05$ & 0.51 & 0.5 & 0.79 \\
\hline $3-29$ & $\begin{array}{l}\text { Old River Outflow Channel near Knox } \\
\text { Landing, La. }\end{array}$ & 0.12 & 0.12 & 0.12 & $<0.05$ & $<0.05$ & $<0.05$ & 0.64 & 0.72 & 0.74 \\
\hline $3-30$ & Mississippi River near St. Francisville, La. & 0.12 & 0.12 & 0.12 & $<0.05$ & $<0.05$ & $<0.05$ & 0.71 & 0.7 & 0.75 \\
\hline 4-01 & Mississippi River below Belle Chasse, La. & 0.12 & 0.12 & 0.12 & $<0.05$ & $<0.05$ & $<0.05$ & 0.79 & 0.75 & 0.65 \\
\hline
\end{tabular}


Table 21.- Concentration data for dissolved trace elements in water samples collected from the Mississippi River and some of its tributaries during the March-April 1989 cruise - Continued

\begin{tabular}{|c|c|c|c|c|c|c|c|}
\hline \multirow{2}{*}{$\begin{array}{l}\text { Date } \\
1989\end{array}$} & \multirow[b]{2}{*}{ Sampling site } & \multicolumn{3}{|c|}{ Vanadium } & \multicolumn{3}{|c|}{ Zinc } \\
\hline & & A & B & Grab & A & B & Grab \\
\hline $3-09$ & Illinois River at Hardin, Ill. & 1.7 & 1.8 & 1.5 & 8.1 & 8.6 & 7.5 \\
\hline $3-10$ & Mississippi River near Winfield, Mo. & 0.65 & 0.72 & 0.62 & 3.6 & 2.2 & 1.5 \\
\hline $3-12$ & Missouri River at Hermann, Mo. & 1.3 & 1.2 & 1.1 & 1.9 & 1.3 & 1.1 \\
\hline $3-13$ & Mississippi River at St. Louis, Mo. & 0.92 & 1 & - & 2.1 & - & - \\
\hline 3-15 & Mississippi River at Thebes, Ill. & 1.1 & 1.2 & 1.1 & 2 & 1.9 & 1.8 \\
\hline $3-16$ & Ohio River at Olmsted, Ill. & 0.33 & 0.33 & 0.28 & 0.17 & 0.33 & 0.093 \\
\hline 3-17 & Mississippi River below Hickman, Ky. & 0.42 & 0.45 & 0.41 & 0.52 & 0.42 & 0.29 \\
\hline $3-19$ & Mississippi River below Fulton, Tenn. & 0.38 & 0.42 & 0.44 & 0.59 & 0.62 & 1 \\
\hline $3-21$ & Mississippi River at Helena, Ark. & 0.62 & 0.85 & 0.82 & - & 0.87 & 1 \\
\hline $3-22$ & White River at Mile 11.5, Ark. & 0.36 & 0.33 & 0.28 & 0.3 & 0.88 & 0.39 \\
\hline $3-23$ & Arkansas River at Pendleton, Ark. & 0.45 & 0.48 & 0.5 & 0.76 & 0.55 & 2.2 \\
\hline $3-24$ & Mississippi River above Arkansas City, Ark. & 0.51 & 0.48 & 0.54 & 1.1 & 6.3 & 0.62 \\
\hline $3-26$ & Yazoo River below Steele Bayou, Miss. & 0.52 & 0.54 & 0.54 & 0.57 & 0.78 & 0.42 \\
\hline 3-27 & Mississippi River below Vicksburg, Miss. & 0.57 & 0.49 & 0.52 & 0.49 & 0.49 & 1.5 \\
\hline $3-29$ & $\begin{array}{l}\text { Old River Outflow Channel near Knox } \\
\text { Landing, La. }\end{array}$ & 0.72 & 0.71 & 0.68 & 4.9 & 2.6 & 1.2 \\
\hline 3-30 & Mississippi River near St. Francisville, La. & 0.71 & 0.69 & 0.69 & 0.81 & 0.91 & 2.2 \\
\hline 4-01 & Mississippi River below Belle Chasse, La. & 0.92 & 0.83 & 0.88 & 2.2 & 1.7 & 3.1 \\
\hline
\end{tabular}

Table 22.- Concentration data for dissolved trace elements in water samples collected from the
Mississippi River and some of its tributaries during the June 1989 cruise

[ A, Composite A; B, Composite B; - , not determined; <, less than the detection limit; All results are in micrograms per liter, except for iron and strontium, which are in milligrams per liter ]

\begin{tabular}{|c|c|c|c|c|c|c|c|c|c|c|}
\hline \multirow{2}{*}{$\begin{array}{l}\text { Date } \\
1989\end{array}$} & \multirow[b]{2}{*}{ Sampling site } & \multicolumn{3}{|c|}{ Aluminum } & \multicolumn{3}{|c|}{ Arsenic } & \multicolumn{3}{|c|}{ Barlum } \\
\hline & & A & B & Grab & A & B & Grab & A & B & Grab \\
\hline $6-04$ & Illinois River at Hardin, III. & 7.2 & -- & 16 & 3.1 & - & 3.1 & 66 & - & 65 \\
\hline $6-05$ & Mississippi River near Winfield, Mo. & 5.3 & 4 & 2.8 & 2.4 & 2.2 & 2.3 & 63 & 63 & 63 \\
\hline $6-07$ & Missouri River at Hermann, Mo. & 6.2 & 5.7 & 4.7 & 2.6 & 2.6 & 2.4 & 88 & 87 & 87 \\
\hline $6-08$ & Mississippi River at St. Louis, Mo. & 3.5 & 3.4 & 2.8 & 2 & 2 & 2.1 & 75 & 75 & 72 \\
\hline $6-10$ & Mississippi River at Thebes, Ill. & 4.6 & 3.4 & 3 & 2.4 & 2.7 & 2.4 & 76 & 76 & 76 \\
\hline $6-11$ & Ohio River at Olmsted, Ill. & 6.1 & 5.8 & 45 & 1.4 & 1.1 & 1.1 & 39 & 39 & 42 \\
\hline $6-12$ & Mississippi River below Hickman, Ky. & 5.2 & 6.7 & 4.9 & 1.5 & 1.5 & 1.3 & 52 & 54 & 49 \\
\hline $6-14$ & Mississippi River at Fulton, Tenn. & 5.3 & 6.7 & 5.1 & 1.3 & 1.4 & 1.3 & 55 & 54 & 55 \\
\hline 6-17 & Mississippi River at Helena, Ark. & 8.7 & 7.1 & 4.4 & 1.5 & 1.2 & 1.3 & 52 & 52 & 51 \\
\hline $6-18$ & White River at Mile 11.5, Ark. & 20 & - & 35 & 0.9 & - & 0.78 & 39 & - & 39 \\
\hline $6-19$ & Arkansas River at Pendleton, Ark. & 66 & - & 2.8 & 1.8 & - & 1.4 & 74 & - & 76 \\
\hline $6-20$ & Mississippi River above Arkansas City, Ark. & 79 & 64 & 4.4 & 1.2 & 1.8 & 1.4 & 50 & 51 & 52 \\
\hline $6-22$ & Yazoo River below Steele Bayou, Miss. & 25 & 23 & 24 & 1.9 & 1.3 & 1.8 & 26 & 25 & 27 \\
\hline $6-23$ & Mississippi River below Vicksburg, Miss. & 4.9 & 4 & 3.3 & 1.3 & 1.2 & 1.8 & 48 & 48 & 48 \\
\hline $6-25$ & $\begin{array}{l}\text { Old River Outflow Channel near Knox } \\
\text { Landing, La. }\end{array}$ & 3.5 & 5.4 & 3.4 & 1 & 1.4 & 1.5 & 48 & 49 & 50 \\
\hline $6-26$ & Mississippi River near St. Francisville, La. & 3.7 & 3.7 & 7.3 & 2.1 & 0.84 & 2 & 48 & 49 & 49 \\
\hline $6-28$ & Mississippi River below Belle Chasse, La. & 4.4 & 4.6 & 3.6 & 1.9 & 2.2 & 1.7 & 50 & 50 & 52 \\
\hline
\end{tabular}


Table 22.- Concentration data for dissolved trace elements in water samples collected from the Mississippi River and some of its tributaries during the June 1989 cruise - Continued

\begin{tabular}{|c|c|c|c|c|c|c|c|c|c|c|}
\hline \multirow{2}{*}{$\begin{array}{l}\text { Date } \\
1989\end{array}$} & \multirow[b]{2}{*}{ Sampling site } & \multicolumn{3}{|c|}{ Beryllium } & \multicolumn{3}{|c|}{ Boron } & \multicolumn{3}{|c|}{ Cadmium } \\
\hline & & $\mathbf{A}$ & B & Grab & $\mathbf{A}$ & B & Grab & A & B & Grab \\
\hline $6-04$ & Illinois River at Hardin, Ill. & 0.043 & - & 0.03 & 150 & - & 150 & $<0.1$ & - & $<0.1$ \\
\hline $6-05$ & Mississippi River near Winfield, Mo. & $<0.02$ & $<0.02$ & 0.027 & 46 & 43 & 46 & $<0.1$ & $<0.1$ & $<0.1$ \\
\hline 6-07 & Missouri River at Hermann, Mo. & 0.033 & $<0.02$ & 0.025 & 110 & 110 & 110 & $<0.1$ & $<0.1$ & $<0.1$ \\
\hline $6-08$ & Mississippi River at St. Louis, Mo. & $<0.02$ & 0.041 & $<0.02$ & 89 & 93 & 87 & $<0.1$ & $<0.1$ & $<0.1$ \\
\hline $6-10$ & Mississippi River at Thebes, Ill. & 0.037 & 0.029 & 0.024 & 94 & 97 & 98 & $<0.1$ & $<0.1$ & $<0.1$ \\
\hline $6-11$ & Ohio River at Olmsted, Ill. & $<0.02$ & $<0.02$ & $<0.02$ & 33 & 32 & 33 & $<0.1$ & $<0.1$ & $<0.1$ \\
\hline $6-12$ & Mississippi River below Hickman, Ky. & $<0.02$ & $<0.02$ & $<0.02$ & 46 & 48 & 42 & $<0.1$ & $<0.1$ & $<0.1$ \\
\hline $6-14$ & Mississippi River at Fulton, Tenn. & $<0.02$ & $<0.02$ & $<0.02$ & 48 & 48 & 48 & $<0.1$ & $<0.1$ & $<0.1$ \\
\hline $6-17$ & Mississippi River at Helena, Ark. & $<0.02$ & $<0.02$ & $<0.02$ & 44 & 43 & 45 & $<0.1$ & $<0.1$ & $<0.1$ \\
\hline 6-18 & White River at Mile 11.5, Ark. & $<0.02$ & - & $<0.02$ & 8.3 & - & 7.9 & $<0.1$ & - & $<0.1$ \\
\hline $6-19$ & Arkansas River at Pendleton, Ark. & $<0.02$ & - & $<0.02$ & 44 & - & 41 & $<0.1$ & - & $<0.1$ \\
\hline $6-20$ & Mississippi River above Arkansas City, Ark. & $<0.02$ & $<0.02$ & 0.022 & 34 & 35 & 35 & $<0.1$ & $<0.1$ & $<0.1$ \\
\hline $6-22$ & Yazoo River below Steele Bayou, Miss. & $<0.02$ & $<0.02$ & $<0.02$ & 16 & 14 & 14 & $<0.1$ & $<0.1$ & $<0.1$ \\
\hline $6-23$ & Mississippi River below Vicksburg, Miss. & $<0.02$ & $<0.02$ & $<0.02$ & 31 & 31 & 32 & $<0.1$ & $<0.1$ & $<0.1$ \\
\hline $6-25$ & $\begin{array}{l}\text { Old River Outflow Channel near Knox } \\
\text { Landing, La. }\end{array}$ & $<0.02$ & $<0.02$ & $<0.02$ & 32 & 32 & 33 & $<0.1$ & $<0.1$ & $<0.1$ \\
\hline $6-26$ & Mississippi River near St. Francisville, La. & $<0.02$ & 0.033 & $<0.02$ & 34 & 34 & 34 & $<0.1$ & $<0.1$ & $<0.1$ \\
\hline $6-28$ & Mississippi River below Belle Chasse, La. & $<0.02$ & $<0.02$ & 0.028 & 32 & 38 & 36 & $<0.1$ & $<0.1$ & $<0.1$ \\
\hline
\end{tabular}

Table 22.- Concentration data for dissolved trace elements in water samples collected from the Mississippi River and some of its tributaries during the June 1989 cruise - Continued

\begin{tabular}{|c|c|c|c|c|c|c|c|c|c|c|}
\hline \multirow{2}{*}{$\begin{array}{l}\text { Date } \\
1989\end{array}$} & \multirow[b]{2}{*}{ Sampling site } & \multicolumn{3}{|c|}{ Chromium } & \multicolumn{3}{|c|}{ Cobait } & \multicolumn{3}{|c|}{ Copper } \\
\hline & & A & B & Grab & A & B & Grab & A & B & Grab \\
\hline$\overline{6-04}$ & Illinois River at Hardin, Ill. & 0.56 & - & 0.66 & 0.73 & - & 0.72 & 2.2 & - & 2.1 \\
\hline $6-05$ & Mississippi River near Winfield, Mo. & 0.39 & 0.4 & 0.44 & 0.44 & 0.4 & 0.44 & 1.5 & 1.4 & 1.3 \\
\hline $6-07$ & Missouri River at Hermann, Mo. & 0.53 & 0.59 & 0.46 & 0.37 & 0.35 & 0.37 & 1.5 & 1.6 & 1.5 \\
\hline $6-08$ & Mississippi River at St. Louis, Mo. & 0.34 & 0.39 & 0.29 & 0.39 & 0.45 & 0.44 & 1.5 & 1.5 & 1.4 \\
\hline $6-10$ & Mississippi River at Thebes, Ill. & 0.35 & 0.43 & 0.36 & 0.41 & 0.43 & 0.42 & 1.6 & 1.6 & 1.5 \\
\hline $6-11$ & Ohio River at Olmsted, Ill. & $<0.2$ & $<0.2$ & $<0.2$ & 0.13 & 0.11 & 0.18 & 1.3 & 1.2 & 1.3 \\
\hline $6-12$ & Mississippi River below Hickman, Ky. & $<0.2$ & $<0.2$ & $<0.2$ & 0.15 & 0.19 & 0.16 & 1.3 & 1.2 & 1.3 \\
\hline 6-14 & Mississippi River at Fulton, Tenn. & $<0.2$ & $<0.2$ & $<0.2$ & 0.14 & 0.16 & 0.18 & 1.3 & 1.2 & 1.3 \\
\hline 6-17 & Mississippi River at Helena, Ark. & $<0.2$ & $<0.2$ & $<0.2$ & 0.11 & 0.13 & 0.093 & 1.2 & 1.2 & 1.2 \\
\hline $6-18$ & White River at Mile 11.5, Ark. & $<0.2$ & - & $<0.2$ & 0.03 & - & 0.024 & 0.48 & - & 0.48 \\
\hline $6-19$ & Arkansas River at Pendleton, Ark. & $<0.2$ & - & $<0.2$ & 0.27 & - & 0.22 & 1.7 & -- & 1.5 \\
\hline $6-20$ & Mississippi River above Arkansas City, Ark. & $<0.2$ & $<0.2$ & $<0.2$ & 0.28 & 0.3 & 0.24 & 1.4 & 1.5 & 1.5 \\
\hline $6-22$ & Yazoo River below Steele Bayou, Miss. & $<0.2$ & $<0.2$ & $<0.2$ & 0.046 & 0.068 & 0.052 & 2 & 1.8 & 1.8 \\
\hline $6-23$ & Mississippi River below Vicksburg, Miss. & $<0.2$ & $<0.2$ & $<0.2$ & 0.22 & 0.2 & 0.2 & 1.4 & 1.3 & 1.4 \\
\hline $6-25$ & $\begin{array}{l}\text { Old River Outflow Channel near Knox } \\
\text { Landing, La. }\end{array}$ & $<0.2$ & $<0.2$ & $<0.2$ & 0.23 & 0.21 & 0.22 & 1.4 & 1.3 & 1.4 \\
\hline $6-26$ & Mississippi River near St. Francisville, La. & $<0.2$ & $<0.2$ & $<0.2$ & 0.28 & 0.24 & 0.27 & 1.5 & 1.5 & 1.5 \\
\hline $6-28$ & Mississippi River below Belle Chasse, La. & $<0.2$ & $<0.2$ & $<0.2$ & 0.22 & 0.27 & 0.32 & 1.7 & 2.4 & 1.6 \\
\hline
\end{tabular}


Table 22.- Concentration data for dissolved trace elements in water samples collected from the Mississippi River and some of its tributaries during the June 1989 cruise - Continued

\begin{tabular}{|c|c|c|c|c|c|c|c|c|c|c|}
\hline \multirow{2}{*}{$\begin{array}{l}\text { Date } \\
1989\end{array}$} & \multirow[b]{2}{*}{ Sampling site } & \multicolumn{3}{|c|}{ Iron } & \multicolumn{3}{|c|}{ Lead } & \multicolumn{3}{|c|}{ Lithium } \\
\hline & & A & B & Grab & A & B & Grab & A & B & Grab \\
\hline $6-04$ & Illinois River at Hardin, Ill. & $<0.005$ & - & $<0.005$ & 0.085 & - & 0.1 & 6.3 & - & 6.6 \\
\hline $6-05$ & Mississippi River near Winfield, Mo. & $<0.005$ & $<0.005$ & $<0.005$ & $<0.06$ & $<0.06$ & $<0.06$ & 4.7 & 4.7 & 5 \\
\hline $6-07$ & Missouri River at Hermann, Mo. & $<0.005$ & $<0.005$ & 0.007 & $<0.06$ & 0.32 & $<0.06$ & 33 & 32 & 32 \\
\hline $6-08$ & Mississippi River at St. Louis, Mo. & $<0.005$ & 0.005 & $<0.005$ & $<0.06$ & $<0.06$ & $<0.06$ & 16 & 17 & 14 \\
\hline $6-10$ & Mississippi River at Thebes, Ill. & $<0.005$ & $<0.005$ & 0.005 & $<0.06$ & $<0.06$ & $<0.06$ & 15 & 17 & 17 \\
\hline $6-11$ & Ohio River at Olmsted, Ill. & $<0.005$ & $<0.005$ & 0.04 & 0.087 & $<0.06$ & 0.07 & 2.2 & 2 & 2.2 \\
\hline $6-12$ & Mississippi River below Hickman, Ky. & $<0.005$ & $<0.005$ & $<0.005$ & 0.076 & $<0.06$ & $<0.06$ & 5.4 & 5.4 & 4.6 \\
\hline $6-14$ & Mississippi River at Fulton, Tenn. & 0.005 & $<0.005$ & $<0.005$ & $<0.06$ & $<0.06$ & $<0.06$ & 5.1 & 5.4 & 5.3 \\
\hline 6-17 & Mississippi River at Helena, Ark. & $<0.005$ & 0.005 & $<0.005$ & $<0.06$ & $<0.06$ & $<0.06$ & 4.5 & 4.4 & 4.5 \\
\hline 6-18 & White River at Mile 11.5, Ark. & 0.024 & - & 0.038 & $<0.06$ & - & $<0.06$ & 0.27 & - & 0.31 \\
\hline $6-19$ & Arkansas River at Pendleton, Ark. & 0.061 & - & $<0.005$ & 0.17 & - & 0.13 & 3.4 & - & 3.3 \\
\hline $6-20$ & Mississippi River above Arkansas City, Ark. & 0.085 & 0.07 & $<0.005$ & 0.18 & 0.15 & 0.16 & 3.4 & 3.7 & 3.4 \\
\hline 6-22 & Yazoo River below Steele Bayou, Miss. & 0.041 & 0.037 & 0.049 & $<0.06$ & 0.068 & 0.069 & 0.73 & 0.65 & 0.6 \\
\hline $6-23$ & Mississippi River below Vicksburg, Miss. & $<0.005$ & 0.006 & $<0.005$ & 0.063 & $<0.06$ & $<0.06$ & 2.9 & 2.9 & 2.9 \\
\hline $6-25$ & $\begin{array}{l}\text { Old River Outflow Channel near Knox } \\
\text { Landing, La. }\end{array}$ & $<0.005$ & $<0.005$ & $<0.005$ & $<0.06$ & $<0.06$ & $<0.06$ & 3.1 & 3 & 3.3 \\
\hline $6-26$ & Mississippi River near St. Francisville, La. & $<0.005$ & $<0.005$ & $<0.005$ & $<0.06$ & $<0.06$ & 1.4 & 3.4 & 3.3 & 3.3 \\
\hline $6-28$ & Mississippi River below Belle Chasse, La. & $<0.005$ & $<0.005$ & $<0.005$ & $<0.06$ & $<0.06$ & 0.096 & 3.4 & 3.6 & 3.5 \\
\hline
\end{tabular}

Table 22.- Concentration data for dissolved trace elements in water samples collected from the Mississippi River and some of its tributaries during the June 1989 cruise - Continued

\begin{tabular}{|c|c|c|c|c|c|c|c|c|c|c|}
\hline \multirow{2}{*}{$\begin{array}{l}\text { Date } \\
1989\end{array}$} & \multirow[b]{2}{*}{ Sampling slte } & \multicolumn{3}{|c|}{ Manganese } & \multicolumn{3}{|c|}{ Molybdenum } & \multicolumn{3}{|c|}{ Nickel } \\
\hline & & A & B & Grab & A & B & Grab & A & B & Grab \\
\hline $6-04$ & Illinois River at Hardin, Ill. & 0.36 & - & 1.1 & 5.7 & - & 6.1 & 14 & - & 15 \\
\hline 6-05 & Mississippi River near Winfield, Mo. & 0.44 & 0.39 & 0.57 & 1.7 & 2 & 1.8 & 7.6 & 7.1 & 7.7 \\
\hline 6-07 & Missouri River at Hermann, Mo. & 0.21 & 0.27 & 0.2 & 3.3 & 3.3 & 3.3 & 8.7 & 8.4 & 8.4 \\
\hline $6-08$ & Mississippi River at St. Louis, Mo. & 0.38 & 0.48 & 0.45 & 3.1 & 3.1 & 2.9 & 8.6 & 8.9 & 8.5 \\
\hline 6-10 & Mississippi River at Thebes, Ill. & 0.81 & 0.37 & 0.66 & 3.1 & 3.2 & 3.2 & 8.6 & 9 & 8.9 \\
\hline $6-11$ & Ohio River at Olmsted, Ill. & 1.4 & 0.54 & 8.3 & 2.4 & 2.1 & 2 & 5.4 & 5.5 & 5.5 \\
\hline $6-12$ & Mississippi River below Hickman, Ky. & 2 & 4.9 & 1.4 & 2.4 & 2.4 & 2.3 & 6.3 & 6.6 & 5.9 \\
\hline 6-14 & Mississippi River at Fulton, Tenn. & 2.2 & 3.9 & 1.3 & 2.2 & 2.2 & 2.4 & 6 & 6.1 & 5.9 \\
\hline $6-17$ & Mississippi River at Helena, Ark. & 0.2 & 0.12 & 0.16 & 2.2 & 2.2 & 2.1 & 5.8 & 5.6 & 5.3 \\
\hline $6-18$ & White River at Mile 11.5, Ark. & 1.5 & - & 2.4 & 0.73 & - & 0.58 & 3.4 & - & 3.6 \\
\hline $6-19$ & Arkansas River at Pendleton, Ark. & 3.4 & -- & 0.87 & 1.1 & - & 0.97 & 5.7 & - & 5.4 \\
\hline $6-20$ & Mississippi River above Arkansas City, Ark. & 4.4 & 4.4 & 0.32 & 1.8 & 1.7 & 1.7 & 5.6 & 5.8 & 5.4 \\
\hline $6-22$ & Yazoo River below Steele Bayou, Miss. & 2.4 & 2.8 & 6.3 & 0.3 & 0.41 & 0.29 & 2.4 & 2.5 & 2.3 \\
\hline $6-23$ & Mississippi River below Vicksburg, Miss. & 0.39 & 0.27 & 0.26 & 1.7 & 1.6 & 1.7 & 4.7 & 5 & 5.2 \\
\hline $6-25$ & $\begin{array}{l}\text { Old River Outflow Channel near Knox } \\
\text { Landing, La. }\end{array}$ & 0.33 & 0.43 & 0.42 & 1.7 & 1.7 & 1.7 & 5.4 & 5.1 & 5.1 \\
\hline $6-26$ & Mississippi River near St. Francisville, La. & 0.27 & 0.3 & 0.35 & 2.1 & 1.8 & 1.8 & 5.3 & 5.3 & 5.6 \\
\hline $6-28$ & Mississippi River below Belle Chasse, La. & 0.28 & 0.25 & 0.95 & 1.9 & 1.8 & 1.9 & 5.2 & 5.5 & 5.8 \\
\hline
\end{tabular}


Table 22.-- Concentration data for dissolved trace elements in water samples collected from the Mississippi River and some of its tributaries during the June 1989 cruise - Continued

\begin{tabular}{|c|c|c|c|c|c|c|c|c|c|c|}
\hline \multirow{2}{*}{$\begin{array}{l}\text { Date } \\
1989\end{array}$} & \multirow[b]{2}{*}{ Sampllng slte } & \multicolumn{3}{|c|}{ Strontlum } & \multicolumn{3}{|c|}{ Thalllum } & \multicolumn{3}{|c|}{ Uranlum } \\
\hline & & A & B & Grab & A & B & Grab & A & B & Grab \\
\hline 6-04 & Illinois River at Hardin, Ill. & 0.22 & - & 0.22 & 0.057 & - & 0.12 & 1.6 & - & 1.9 \\
\hline $6-05$ & Mississippi River near Winfield, Mo. & 0.094 & 0.095 & 0.091 & 0.054 & $<0.05$ & 0.064 & 1.4 & 1.3 & 1.3 \\
\hline $6-07$ & Missouri River at Hermann, Mo. & 0.4 & 0.4 & 0.4 & 0.063 & $<0.05$ & 0.065 & 4.1 & 4.5 & 4.3 \\
\hline $6-08$ & Mississippi River at St. Louis, Mo. & 0.23 & 0.23 & 0.2 & $<0.05$ & 0.054 & $<0.05$ & 2.5 & 2.6 & 2.3 \\
\hline $6-10$ & Mississippi River at Thebes, Ill. & 0.22 & 0.22 & 0.22 & 0.053 & $<0.05$ & 0.06 & 2.2 & 2.5 & 2.4 \\
\hline $6-11$ & Ohio River at Olmsted, Ill. & 0.17 & 0.17 & 0.2 & 0.091 & 0.054 & $<0.05$ & 0.55 & 0.48 & 0.4 \\
\hline $6-12$ & Mississippi River below Hickman, Ky. & 0.18 & 0.18 & 0.18 & $<0.05$ & 0.051 & $<0.05$ & 0.99 & 0.99 & 0.92 \\
\hline 6-14 & Mississippi River at Fulton, Tenn. & 0.18 & 0.18 & 0.18 & $<0.05$ & $<0.05$ & $<0.05$ & 0.93 & 0.91 & 1.1 \\
\hline 6-17 & Mississippi River at Helena, Ark. & 0.17 & 0.18 & 0.19 & $<0.05$ & $<0.05$ & $<0.05$ & 0.74 & 0.7 & 0.82 \\
\hline 6-18 & White River at Mile 11.5, Ark. & 0.096 & - & 0.047 & $<0.05$ & - & $<0.05$ & 0.22 & - & 0.3 \\
\hline $6-19$ & Arkansas River at Pendleton, Ark. & 0.27 & - & 0.27 & 0.083 & - & 0.063 & 0.9 & - & 0.99 \\
\hline $6-20$ & Mississippi River above Arkansas City, Ark. & 0.21 & 0.16 & 0.17 & $<0.05$ & 0.063 & $<0.05$ & 0.76 & 0.84 & 0.68 \\
\hline 6-22 & Yazoo River below Steele Bayou, Miss. & 0.031 & 0.093 & 0.031 & $<0.05$ & $<0.05$ & $<0.05$ & $<0.06$ & 0.11 & $<0.06$ \\
\hline $6-23$ & Mississippi River below Vicksburg, Miss. & 0.15 & 0.16 & 0.16 & $<0.05$ & $<0.05$ & 0.068 & 0.64 & 0.54 & 0.56 \\
\hline $6-25$ & $\begin{array}{l}\text { Old River Outflow Channel near Knox } \\
\text { Landing, La. }\end{array}$ & 0.15 & 0.15 & 0.15 & $<0.05$ & $<0.05$ & $<0.05$ & 0.68 & 0.56 & 0.59 \\
\hline $6-26$ & Mississippi River near St. Francisvi & 0.15 & 0.16 & 0.16 & $<0.05$ & $<0.05$ & $<0.05$ & 0.72 & 0.6 & 0.69 \\
\hline $6-28$ & Mississippi River below Belle Chasse, La. & 0.14 & 0.13 & 0.15 & $<0.05$ & $<0.05$ & $<0.05$ & 0.73 & 0.68 & 0.76 \\
\hline
\end{tabular}

Table 22.- Concentration data for dissolved trace elements in water samples collected from the Mississippi River and some of its tributaries during the June 1989 cruise - Continued

\begin{tabular}{|c|c|c|c|c|c|c|c|}
\hline \multirow{2}{*}{$\begin{array}{l}\text { Date } \\
1989\end{array}$} & \multirow[b]{2}{*}{ Sampllng site } & \multicolumn{3}{|c|}{ Vanadium } & \multicolumn{3}{|c|}{ Zinc } \\
\hline & & A & B & Grab & A & B & Grab \\
\hline $6-04$ & Illinois River at Hardin, Ill. & $\overline{3.4}$ & - & 3.4 & 4.3 & - & 4.8 \\
\hline $6-05$ & Mississippi River near Winfield, Mo. & 2.2 & 2.2 & 2.2 & 4.4 & 2.2 & 2.3 \\
\hline 6-07 & Missouri River at Hermann, Mo. & 2.7 & 2.6 & 2.4 & 3.3 & 7.5 & 1.9 \\
\hline $6-08$ & Mississippi River at St. Louis, Mo. & 2.6 & 2.7 & 2.6 & 4.5 & 3.2 & 3.8 \\
\hline $6-10$ & Mississippi River at Thebes, Ill. & 2.6 & 2.7 & 2.7 & 4.5 & 1.7 & 2.2 \\
\hline $6-11$ & Ohio River at Olmsted, Ill. & 0.7 & 0.62 & 0.62 & 1.7 & 3 & 2.8 \\
\hline $6-12$ & Mississippi River below Hickman, Ky. & 1.2 & 1.3 & 1 & 2.2 & 1.3 & 1.1 \\
\hline $6-14$ & Mississippi River at Fulton, Tenn. & 1.1 & 1.1 & 1.2 & 3.5 & 1.7 & 1 \\
\hline $6-17$ & Mississippi River at Helena, Ark. & 1.1 & 1.1 & 1.1 & 0.82 & 0.97 & 0.79 \\
\hline $6-18$ & White River at Mile 11.5, Ark. & 0.65 & - & 0.65 & 1.1 & - & 0.95 \\
\hline $6-19$ & Arkansas River at Pendleton, Ark. & 1.7 & - & 1.4 & 2.6 & - & 1.2 \\
\hline $6-20$ & Mississippi River above Arkansas City, Ark. & 1.3 & 1.2 & 1.1 & 2.4 & 2.1 & 2.7 \\
\hline $6-22$ & Yazoo River below Steele Bayou, Miss. & 1 & 1 & 0.91 & 3.3 & 0.58 & 17 \\
\hline $6-23$ & Mississippi River below Vicksburg, Miss. & 1 & 1 & 1 & 5.3 & 2.3 & 1.2 \\
\hline $6-25$ & $\begin{array}{l}\text { Old River Outflow Channel near Knox } \\
\text { Landing, La. }\end{array}$ & 1.1 & 1.1 & 1.1 & 1.8 & 1.1 & 2.7 \\
\hline $6-26$ & Mississippi River near St. Francisville, La. & 1.4 & 1.2 & 1.2 & 2.3 & 1.7 & 3 \\
\hline $6-28$ & Mississippi River below Belle Chasse, La. & 1.4 & 1.4 & 1.4 & 1.5 & 1.9 & 6.4 \\
\hline
\end{tabular}


Table 23.-- Concentration data for dissolved trace elements in water samples collected from the Mississippi River and some of its tributaries during the February-March 1990 cruise

[ A, Composite A; B, Composite B; --, not determined; <, less than the detection limit; All results are in micrograms per liter, except for iron and strontium, which are in milligrams per liter ]

\begin{tabular}{|c|c|c|c|c|c|c|c|c|c|c|}
\hline \multirow{2}{*}{$\begin{array}{l}\text { Date } \\
1990\end{array}$} & \multirow[b]{2}{*}{ Sampling site } & \multicolumn{3}{|c|}{ Aluminum } & \multicolumn{3}{|c|}{ Arsenic } & \multicolumn{3}{|c|}{ Barium } \\
\hline & & A & B & Grab & A & B & Grab & A & $\mathbf{B}$ & Grab \\
\hline $2-23$ & Cumberland River near Smithland, Ky. & 15 & 12 & 17 & 0.65 & $<0.6$ & $<0.6$ & 23 & 23 & 22 \\
\hline $2-24$ & Tennessee River near Calvert City, Ky. & 26 & 28 & 29 & $<0.6$ & 0.61 & $<0.6$ & 20 & 20 & 20 \\
\hline $2-25$ & Mississippi River near Cache, Ill. & 6.7 & - & 13 & 1.6 & -- & 1.7 & 65 & - & 66 \\
\hline $2-28$ & Wabash River near New Haven, Ill. & 4.7 & 4.8 & 7.1 & 0.77 & $<0.6$ & 1.1 & 40 & 41 & 41 \\
\hline 3-01 & Ohio River at Uniontown, Ky. & 13 & 11 & 13 & 0.6 & $<0.6$ & $<0.6$ & 31 & 32 & 31 \\
\hline $3-03$ & Ohio River at Olmsted, Ill. & 6.5 & 6.1 & 6.9 & 0.62 & $<0.6$ & 0.63 & 28 & 28 & 32 \\
\hline 3-04 & Mississippi River below Hickman, Ky. & 7.3 & 5 & 4.9 & $<0.6$ & 0.7 & 0.84 & 37 & 37 & 40 \\
\hline 3-05 & Mississippi River below Fulton, Tenn. & - & 4.9 & 5.9 & 0.74 & $<0.6$ & $<0.6$ & 36 & 36 & 37 \\
\hline 3-07 & Mississippi River at Helena, Ark. & 4 & - & 3.9 & 1.4 & 1.1 & 1.4 & 41 & 41 & 39 \\
\hline 3-07 & Arkansas River at Mile 1.0, Ark. & 2.2 & - & - & 0.79 & - & - & 52 & -- & - \\
\hline 3-08 & Mississippi River above Arkansas City, Ark. & 3.2 & 3.1 & 3.3 & 0.81 & 0.89 & 0.85 & 40 & 40 & 41 \\
\hline 3-08 & White River at Mile 1.0, Ark. & 8.5 & - & - & 0.72 & - & - & 32 & - & - \\
\hline $3-10$ & Yazoo River at Mile 1.0, Miss. & 18 & - & - & 1.1 & -- & - & 32 & - & - \\
\hline $3-10$ & Mississippi River below Vicksburg, Miss. & 3.7 & 4.1 & 360 & 0.87 & 1.1 & 0.98 & 40 & 40 & 46 \\
\hline 3-12 & Mississippi River near St. Francisville, La. & 2.9 & 4.1 & 2.5 & 0.81 & 0.75 & 0.88 & 42 & 42 & 42 \\
\hline 3-14 & Mississippi River below Belle Chasse, La. & 3.2 & 3.2 & 6.4 & 1.2 & 1.5 & 1 & 44 & 43 & 45 \\
\hline
\end{tabular}

Table 23.- Concentration data for dissolved trace elements in water samples collected from the Mississippi River and some of its tributaries during the February-March 1990 cruise - Continued

\begin{tabular}{|c|c|c|c|c|c|c|c|c|c|c|}
\hline \multirow{2}{*}{$\begin{array}{l}\text { Date } \\
1990\end{array}$} & \multirow[b]{2}{*}{ Sampling slte } & \multicolumn{3}{|c|}{ Beryllium } & \multicolumn{3}{|c|}{ Boron } & \multicolumn{3}{|c|}{ Cadmlum } \\
\hline & & A & B & Grab & A & B & Grab & A & B & Grab \\
\hline $2-23$ & Cumberland River near Smithland, Ky. & $<0.02$ & $<0.02$ & $<0.02$ & 13 & 13 & 13 & $<0.1$ & $<0.1$ & $<0.1$ \\
\hline $2-24$ & Tennessee River near Calvert City, Ky. & $<0.02$ & $<0.02$ & $<0.02$ & 7.2 & 7.4 & 6.7 & $<0.1$ & $<0.1$ & $<0.1$ \\
\hline $2-25$ & Mississippi River near Cache, Ill. & $<0.02$ & - & $<0.02$ & 61 & - & 65 & $<0.1$ & - & $<0.1$ \\
\hline $2-28$ & Wabash River near New Haven, Ill. & $<0.02$ & $<0.02$ & $<0.02$ & 35 & 40 & 39 & $<0.1$ & $<0.1$ & $<0.1$ \\
\hline $3-01$ & Ohio River at Uniontown, Ky. & $<0.02$ & $<0.02$ & $<0.02$ & 26 & 25 & 24 & $\varangle 0.1$ & $<0.1$ & $<0.1$ \\
\hline 3-03 & Ohio River at Olmsted, Ill. & $<0.02$ & $<0.02$ & $<0.02$ & 22 & 20 & 26 & $<0.1$ & $<0.1$ & $<0.1$ \\
\hline 3-04 & Mississippi River below Hickman, Ky. & $<0.02$ & $<0.02$ & $<0.02$ & 25 & 25 & 28 & $<0.1$ & $<0.1$ & $<0.1$ \\
\hline 3-05 & Mississippi River below Fulton, Tenn. & $<0.02$ & $<0.02$ & $<0.02$ & 27 & 25 & 28 & $<0.1$ & $<0.1$ & $<0.1$ \\
\hline 3-07 & Mississippi River at Helena, Ark. & 0.026 & $<0.02$ & 0.04 & 25 & 22 & 27 & $<0.1$ & $<0.1$ & $<0.1$ \\
\hline 3-07 & Arkansas River at Mile 1.0, Ark. & $<0.02$ & -- & -- & 26 & - & -- & $<0.1$ & - & - \\
\hline $3-08$ & Mississippi River above Arkansas City, Ark. & $<0.02$ & 0.039 & $<0.02$ & 22 & 23 & 24 & $<0.1$ & $<0.1$ & $<0.1$ \\
\hline 3-08 & White River at Mile 1.0, Ark. & $<0.02$ & -- & - & 3.9 & - & - & $<0.1$ & - & - \\
\hline $3-10$ & Yazoo River at Mile 1.0, Miss. & $<0.02$ & -- & -- & 8.8 & - & - & $<0.1$ & - & - \\
\hline $3-10$ & Mississippi River below Vicksburg, Miss. & $<0.02$ & $<0.02$ & 0.068 & 21 & 22 & 24 & $<0.1$ & $<0.1$ & $<0.1$ \\
\hline 3-12 & Mississippi River near St. Francisville, La. & $<0.02$ & $<0.02$ & $<0.02$ & 21 & 22 & 21 & $<0.1$ & $<0.1$ & $<0.1$ \\
\hline $3-14$ & Mississippi River below Belle Chasse, La. & 0.054 & 0.021 & $\ll .02$ & 21 & 23 & 22 & $<0.1$ & $<0.1$ & $<0.1$ \\
\hline
\end{tabular}


Table 23.- Concentration data for dissolved trace elements in water samples collected from the Mississippi River and some of its tributaries during the February-March 1990 cruise - Continued

\begin{tabular}{|c|c|c|c|c|c|c|c|c|c|c|}
\hline \multirow{2}{*}{$\begin{array}{l}\text { Date } \\
1990 \\
\end{array}$} & \multirow[b]{2}{*}{ Sampling site } & \multicolumn{3}{|c|}{ Chromium } & \multicolumn{3}{|c|}{ Cobalt } & \multicolumn{3}{|c|}{ Copper } \\
\hline & & A & B & Grab & A & B & Grab & A & B & Grab \\
\hline $2-23$ & Cumberland River near Smithland, Ky. & $<0.2$ & $<0.2$ & 0.65 & 0.29 & 0.27 & 0.27 & 0.74 & 0.83 & 0.61 \\
\hline $2-24$ & Tennessee River near Calvert City, Ky. & $<0.2$ & $<0.2$ & 0.51 & 0.2 & 0.18 & 0.19 & 0.77 & 0.7 & 0.75 \\
\hline $2-25$ & Mississippi River near Cache, IIl. & 0.58 & -- & 0.61 & 0.5 & - & 0.56 & 1.8 & - & 1.7 \\
\hline $2-28$ & Wabash River near New Haven, Ill. & $<0.2$ & $<0.2$ & $<0.2$ & 0.52 & 0.49 & 0.52 & 1.8 & 1.6 & 1.6 \\
\hline $3-01$ & Ohio River at Uniontown, Ky. & $<0.2$ & $<0.2$ & $<0.2$ & 0.32 & 0.31 & 0.31 & 1.4 & 1.6 & 1.4 \\
\hline 3-03 & Ohio River at Olmsted, Ill. & $<0.2$ & $<0.2$ & $<0.2$ & 0.23 & 0.21 & 0.26 & 1.4 & 1.2 & 1.3 \\
\hline 3-04 & Mississippi River below Hickman, Ky. & $<0.2$ & $<0.2$ & $<0.2$ & 0.27 & 0.26 & 0.28 & 1.3 & 1.2 & 1.3 \\
\hline 3-05 & Mississippi River below Fulton, Tenn. & 0.3 & $<0.2$ & $<0.2$ & 0.29 & 0.25 & 0.26 & 1.4 & 1.4 & 1.1 \\
\hline 3-07 & Mississippi River at Helena, Ark. & $<0.2$ & 0.4 & $<0.2$ & 0.28 & 0.27 & 0.32 & 1.5 & 1.7 & 1.3 \\
\hline 3-07 & Arkansas River at Mile 1.0, Ark. & $<0.2$ & - & -- & 0.16 & - & -- & 0.87 & - & - \\
\hline 3-08 & Mississippi River above Arkansas City, Ark. & $<0.2$ & $<0.2$ & $<0.2$ & 0.25 & 0.24 & 0.29 & 1.2 & 1.2 & 1.2 \\
\hline $3-08$ & White River at Mile 1.0, Ark. & $<0.2$ & - & - & 0.12 & -- & - & 0.74 & - & - \\
\hline $3-10$ & Yazoo River at Mile 1.0, Miss. & $<0.2$ & - & - & 0.025 & - & - & 1.3 & - & - \\
\hline $3-10$ & Mississippi River below Vicksburg, Miss. & $<0.2$ & $<0.2$ & 0.23 & 0.22 & 0.23 & 0.48 & 1.5 & 1.5 & 1.7 \\
\hline 3-12 & Mississippi River near St. Francisville, La. & $<0.2$ & $<0.2$ & $<0.2$ & 0.22 & 0.26 & 0.22 & 1.4 & 1.9 & 1.3 \\
\hline $3-14$ & Mississippi River below Belle Chasse, La. & $<0.2$ & $<0.2$ & $<0.2$ & 0.26 & 0.25 & 0.26 & 3 & 1.4 & 1.4 \\
\hline
\end{tabular}

Table 23.- Concentration data for dissolved trace elements in water samples collected from the Mississippi River and some of its tributaries during the February-March 1990 cruise - Continued

\begin{tabular}{|c|c|c|c|c|c|c|c|c|c|c|}
\hline \multirow{2}{*}{$\begin{array}{l}\text { Date } \\
1990\end{array}$} & \multirow[b]{2}{*}{ Sampling site } & \multicolumn{3}{|c|}{ Iron } & \multicolumn{3}{|c|}{ Lead } & \multicolumn{3}{|c|}{ Lithium } \\
\hline & & A & B & Grab & A & $\mathbf{B}$ & Grab & A & $\mathbf{B}$ & Grab \\
\hline $2-23$ & Cumberland River near Smithland, Ky. & 0.015 & 0.009 & 0.014 & $<0.06$ & $<0.06$ & $<0.06$ & 0.74 & 0.73 & 0.77 \\
\hline $2-24$ & Tennessee River near Calvert City, Ky. & 0.018 & 0.02 & 0.024 & $<0.06$ & $<0.06$ & $<0.06$ & 0.62 & 0.6 & 0.61 \\
\hline $2-25$ & Mississippi River near Cache, Ill. & 0.006 & - & 0.014 & $<0.06$ & - & $<0.06$ & 8.6 & - & 9.3 \\
\hline $2-28$ & Wabash River near New Haven, Ill. & 0.005 & 0.005 & 0.011 & $<0.06$ & $<0.06$ & $<0.06$ & 1.5 & 1.6 & 1.6 \\
\hline 3-01 & Ohio River at Uniontown, Ky. & 0.007 & 0.016 & 0.009 & $<0.06$ & $<0.06$ & $<0.06$ & 2.9 & 2.6 & 2.6 \\
\hline $3-03$ & Ohio River at Olmsted, Ill. & $<0.005$ & 0.013 & 0.006 & 0.092 & $<0.06$ & 0.064 & 1.7 & 1.6 & 2 \\
\hline 3-04 & Mississippi River below Hickman, Ky. & 0.006 & $<0.005$ & $<0.005$ & 0.1 & 0.07 & 0.12 & 2.7 & 2.6 & 3.2 \\
\hline 3-05 & Mississippi River below Fulton, Tenn. & 0.054 & 0.005 & $<0.005$ & 0.13 & 0.1 & $<0.06$ & 2.6 & 2.6 & 2.8 \\
\hline 3-07 & Mississippi River at Helena, Ark. & 0.006 & 0.015 & 0.005 & $<0.06$ & 0.079 & $<0.06$ & 2.5 & 2.4 & 2.7 \\
\hline 3-07 & Arkansas River at Mile 1.0, Ark. & $<0.005$ & -- & -- & $<0.06$ & - & - & 2.4 & - & - \\
\hline $3-08$ & Mississippi River above Arkansas City, Ark. & 0.007 & 0.014 & 0.012 & $<0.06$ & $<0.06$ & 0.076 & 2.3 & 2.2 & 2.5 \\
\hline 3-08 & White River at Mile 1.0, Ark. & 0.016 & -- & -- & $<0.06$ & - & - & 0.18 & - & - \\
\hline $3-10$ & Yazoo River at Mile 1.0, Miss. & 0.031 & - & - & $<0.06$ & - & - & 0.95 & - & - \\
\hline $3-10$ & Mississippi River below Vicksburg, Miss. & $<0.005$ & 0.011 & 0.4 & $<0.06$ & $<0.06$ & 0.48 & 2.2 & 2.1 & 2.7 \\
\hline $3-12$ & Mississippi River near St. Francisville, La. & $<0.005$ & $<0.005$ & $<0.005$ & $<0.06$ & $<0.06$ & $<0.06$ & 2.1 & 2.2 & 2 \\
\hline 3-14 & Mississippi River below Belle Chasse, La. & 0.006 & $<0.005$ & 0.005 & $<0.06$ & $<0.06$ & 0.072 & 2.1 & 2.2 & 2.2 \\
\hline
\end{tabular}


Table 23.- Concentration data for dissolved trace elements in water samples collected from the Mississippi River and some of its tributaries during the February-March 1990 cruise - Continued

\begin{tabular}{|c|c|c|c|c|c|c|c|c|c|c|}
\hline \multirow{2}{*}{$\begin{array}{l}\text { Date } \\
1990\end{array}$} & \multirow[b]{2}{*}{ Sampllng slte } & \multicolumn{3}{|c|}{ Manganese } & \multicolumn{3}{|c|}{ Molybdenum } & \multicolumn{3}{|c|}{ NIckel } \\
\hline & & A & B & Grab & $\mathbf{A}$ & B & Grab & A & $\mathbf{B}$ & Grab \\
\hline $2-23$ & Cumberland River near Smithland, Ky. & 1.5 & 1.3 & 2 & 0.8 & 0.74 & 0.96 & 6 & 5.8 & 5.1 \\
\hline $2-24$ & Tennessee River near Calvert City, Ky. & 1.1 & 1.1 & 5.4 & 0.26 & 0.16 & 0.62 & 3.4 & 3.1 & 3 \\
\hline $2-25$ & Mississippi River near Cache, Ill. & 1.3 & -- & 1.1 & 2.5 & - & 2.5 & 8.8 & - & 9.7 \\
\hline $2-28$ & Wabash River near New Haven, Ill. & 3.4 & 3.6 & 2.8 & 1.7 & 1.7 & 1.8 & 9.1 & 9.9 & 9.5 \\
\hline 3-01 & Ohio River at Uniontown, Ky. & 6.3 & 5.7 & 3.9 & 1.6 & 1.5 & 1.6 & 6.8 & 6.5 & 6.1 \\
\hline 3-03 & Ohio River at Olmsted, Ill. & 3.7 & 3.6 & 2.9 & 1.4 & 1.2 & 1.5 & 6 & 5.7 & 6.5 \\
\hline 3-04 & Mississippi River below Hickman, Ky. & 3.1 & 3.1 & 5.5 & 1.3 & 1.4 & 1.5 & 6.3 & 5.9 & 6.6 \\
\hline 3-05 & Mississippi River below Fulton, Tenn. & 4.4 & 1.8 & 3.3 & 1.3 & 1.3 & 1.2 & 6 & 6.1 & 5.8 \\
\hline 3-07 & Mississippi River at Helena, Ark. & 1.5 & 1.7 & 1.1 & 1.3 & 1.2 & 1.2 & 6.1 & 5.5 & 5.9 \\
\hline 3-07 & Arkansas River at Mile 1.0, Ark. & 0.71 & - & - & 0.26 & - & -- & 4.3 & - & - \\
\hline 3-08 & Mississippi River above Arkansas City, Ark. & 1.4 & 1.5 & 1.8 & 1 & 0.94 & 0.91 & 5.1 & 5.1 & 5.3 \\
\hline 3-08 & White River at Mile 1.0, Ark. & 28 & - & - & $<0.09$ & - & - & 3.1 & - & - \\
\hline 3-10 & Yazoo River at Mile 1.0, Miss. & 9.4 & - & - & $<0.09$ & -- & -- & 2.4 & -- & - \\
\hline 3-10 & Mississippi River below Vicksburg, Miss. & 1 & 1.1 & 22 & 0.93 & 0.99 & 0.93 & 4.7 & 4.8 & 5.8 \\
\hline 3-12 & Mississippi River near St. Francisville, La. & 1.8 & 1.7 & 2.3 & 1 & 0.91 & 0.86 & 4.7 & 5.5 & 4.9 \\
\hline 3-14 & Mississippi River below Belle Chasse, La. & 6.3 & 6.2 & 6.9 & 0.97 & 1 & 1 & 4.8 & 5 & 4.9 \\
\hline
\end{tabular}

Table 23.- Concentration data for dissolved trace elements in water samples collected from the Mississippi River and some of its tributaries during the February-March 1990 cruise - Continued

\begin{tabular}{|c|c|c|c|c|c|c|c|c|c|c|}
\hline \multirow{2}{*}{$\begin{array}{l}\text { Date } \\
1990\end{array}$} & \multirow[b]{2}{*}{ Sampllng slte } & \multicolumn{3}{|c|}{ Strontium } & \multicolumn{3}{|c|}{ Thallium } & \multicolumn{3}{|c|}{ Uranlum } \\
\hline & & A & B & Grab & A & B & Grab & A & B & Grab \\
\hline $2-23$ & Cumberland River near Smithland, Ky. & 0.088 & 0.082 & 0.079 & $<0.05$ & $<0.05$ & 0.052 & $<0.06$ & $<0.06$ & 0.13 \\
\hline $2-24$ & Tennessee River near Calvert City, Ky. & 0.046 & 0.045 & 0.26 & $<0.05$ & $<0.05$ & $<0.05$ & $<0.06$ & $<0.06$ & $<0.06$ \\
\hline $2-25$ & Mississippi River near Cache, Ill. & 0.16 & -- & 0.19 & $<0.05$ & - & $<0.05$ & 1.9 & - & 2.1 \\
\hline $2-28$ & Wabash River near New Haven, Ill. & 0.11 & 0.11 & 0.13 & $<0.05$ & $<0.05$ & $<0.05$ & 0.7 & 0.79 & 0.83 \\
\hline 3-01 & Ohio River at Uniontown, Ky. & 0.18 & 0.17 & 0.19 & $<0.05$ & 0.05 & 0.083 & 0.31 & 0.15 & 0.44 \\
\hline 3-03 & Ohio River at Olmsted, Ill. & 0.11 & 0.12 & 0.15 & $<0.05$ & $<0.05$ & $<0.05$ & 0.42 & 0.37 & 0.45 \\
\hline 3-04 & Mississippi River below Hickman, Ky. & 0.13 & 0.13 & 0.14 & $<0.05$ & $<0.05$ & $<0.05$ & 0.69 & 0.65 & 0.69 \\
\hline 3-05 & Mississippi River below Fulton, Tenn. & 0.11 & 0.13 & 0.14 & $<0.05$ & $<0.05$ & $<0.05$ & 0.62 & 0.65 & 0.71 \\
\hline 3-07 & Mississippi River at Helena, Ark. & 0.13 & 0.11 & 0.12 & $<0.05$ & $<0.05$ & $<0.05$ & 0.75 & 0.58 & 0.61 \\
\hline 3-07 & Arkansas River at Mile 1.0, Ark. & 0.18 & - & -- & $<0.05$ & -- & - & 0.34 & - & -- \\
\hline 3-08 & Mississippi River above Arkansas City, Ark. & 0.12 & 0.12 & 0.11 & $<0.05$ & $<0.05$ & $<0.05$ & 0.68 & 0.6 & 0.61 \\
\hline $3-08$ & White River at Mile 1.0, Ark. & 0.025 & - & - & $<0.05$ & -- & - & $<0.06$ & - & - \\
\hline $3-10$ & Yazoo River at Mile 1.0, Miss. & 0.054 & - & - & $<0.05$ & -- & - & $<0.06$ & - & - \\
\hline $3-10$ & Mississippi River below Vicksburg, Miss. & 0.1 & 0.098 & 0.12 & $<0.05$ & $<0.05$ & $<0.05$ & 0.53 & 0.54 & 0.69 \\
\hline $3-12$ & Mississippi River near St. Francisville, La. & 0.11 & 0.11 & 0.11 & $<0.05$ & $<0.05$ & $<0.05$ & 0.52 & 0.57 & 0.54 \\
\hline 3-14 & Mississippi River below Belle Chasse, La. & 0.11 & 0.11 & 0.11 & $<0.05$ & $<0.05$ & $<0.05$ & 0.77 & 0.67 & 0.67 \\
\hline
\end{tabular}


Table 23.-- Concentration data for dissolved trace elements in water samples collected from the Mississippi River and some of its tributaries during the February-March 1990 cruise - Continued

\begin{tabular}{|c|c|c|c|c|c|c|c|}
\hline \multirow{2}{*}{$\begin{array}{l}\text { Date } \\
1990\end{array}$} & \multirow[b]{2}{*}{ Sampling site } & \multicolumn{3}{|c|}{ Vanadium } & \multicolumn{3}{|c|}{ Zinc } \\
\hline & & A & B & Grab & $\mathbf{A}$ & B & Grab \\
\hline $2-23$ & Cumberland River near Smithland, Ky. & 0.56 & 0.57 & 0.72 & 2.4 & 1.7 & 0.53 \\
\hline $2-24$ & Tennessee River near Calvert City, Ky. & 0.52 & 0.53 & 0.57 & 2.3 & 1.1 & 1.2 \\
\hline $2-25$ & Mississippi River near Cache, Ill. & 1.5 & - & 1.6 & 3 & - & 3.7 \\
\hline $2-28$ & Wabash River near New Haven, 111 . & 0.46 & 0.53 & 0.58 & 1.3 & 1.9 & 1.3 \\
\hline $3-01$ & Ohio River at Uniontown, Ky. & 0.39 & 0.33 & 0.3 & 2.1 & 2.7 & 2 \\
\hline $3-03$ & Ohio River at Olmsted, Ill. & 0.41 & 0.34 & 0.33 & 4.6 & 1.7 & 1.3 \\
\hline 3-04 & Mississippi River below Hickman, Ky. & 0.51 & 0.58 & 0.55 & 1.4 & 1.5 & 1.5 \\
\hline 3-05 & Mississippi River below Fulton, Tenn. & 0.73 & 0.59 & 0.63 & 1.5 & 3 & 1.2 \\
\hline 3-07 & Mississippi River at Helena, Ark. & 0.71 & 0.73 & 0.52 & 1.3 & 3.2 & 1.2 \\
\hline 3-07 & Arkansas River at Mile 1.0, Ark. & 0.071 & - & - & 1.1 & - & - \\
\hline 3-08 & Mississippi River above Arkansas City, Ark. & 0.54 & 0.54 & 0.63 & 1.3 & 1.5 & 2.9 \\
\hline $3-08$ & White River at Mile 1.0, Ark. & $<0.07$ & - & - & 1 & - & - \\
\hline $3-10$ & Yazoo River at Mile 1.0, Miss. & $<0.07$ & -- & -- & 0.63 & - & - \\
\hline $3-10$ & Mississippi River below Vicksburg, Miss. & 0.58 & 0.57 & 1.2 & 1.6 & 2.5 & 3.5 \\
\hline $3-12$ & Mississippi River near St. Francisville, La. & 0.58 & 0.58 & 0.56 & 0.93 & 12 & 1.4 \\
\hline $3-14$ & Mississippi River below Belle Chasse, La. & 0.81 & 0.83 & 0.83 & 2.3 & 1.7 & 1.9 \\
\hline
\end{tabular}

Table 24.- Concentration data for dissolved trace elements in water samples collected from the Mississippi River and some of its tributaries during the May-June 1990 cruise

[ A, Composite A; B, Composite B; --, not determined; <, less than the detection limit; All results are in micrograms per liter, except for iron and strontium, which are in milligrams per liter ]

\begin{tabular}{|c|c|c|c|c|c|c|c|c|c|c|}
\hline \multirow{2}{*}{$\begin{array}{l}\text { Date } \\
1990\end{array}$} & \multirow[b]{2}{*}{ Sampling site } & \multicolumn{3}{|c|}{ Aluminum } & \multicolumn{3}{|c|}{ Arsenic } & \multicolumn{3}{|c|}{ Barium } \\
\hline & & A & $\mathbf{B}$ & Grab & A & B & Grab & A & B & Grab \\
\hline $6-07$ & Illinois River at Valley City, Ill. & 10 & 10 & 2.9 & 0.71 & 0.71 & $<0.6$ & 64 & 68 & 75 \\
\hline $6-10$ & Missouri River at St. Charles, Mo. ${ }^{1}$ & 39 & -- & - & 0.89 & - & 0.63 & 81 & - & 99 \\
\hline $6-11$ & Mississippi River below Grafton, $\mathrm{Il}$. & 5.8 & 5.8 & 17 & 1.0 & 1 & 0.78 & 76 & 78 & 78 \\
\hline $6-13$ & Mississippi River at Thebes, $\mathrm{Ill}$. & 11 & 11 & 6.4 & 1.7 & 2.3 & 1.4 & 78 & 77 & 83 \\
\hline $6-14$ & Ohio River at Olmsted, Ill. & 11 & 11 & 7.6 & 1.1 & $<0.6$ & 0.92 & 30 & 32 & 32 \\
\hline $6-18$ & Mississippi River below Memphis, Tenn. & 11 & 15 & 13 & 0.94 & 0.94 & 1.2 & 59 & 64 & 62 \\
\hline $6-19$ & White River at Mile 1.0, Ark. & - & - & 1.4 & - & -- & 2.7 & - & - & 35 \\
\hline $6-19$ & Arkansas River at Mile 1.0, Ark. & - & - & 2.6 & - & - & 1.9 & - & - & 55 \\
\hline 6-20 & Mississippi River below Arkansas City, Ark. & 9.2 & 14 & 3.7 & 1.0 & 1 & 2.1 & 55 & 64 & 59 \\
\hline $6-22$ & Yazoo River below Steele Bayou, Miss. & 3.5 & 3.5 & 2.3 & 4.4 & 4.2 & 5.1 & 33 & 35 & 37 \\
\hline $6-23$ & Mississippi River below Vicksburg, Miss. & 4.8 & 4.8 & 8.3 & 1.5 & 1.5 & 2.2 & 56 & 57 & 57 \\
\hline $6-25$ & Mississippi River near St. Francisville, La. & 13 & 13 & 3.5 & 1.9 & 1.9 & 1.6 & 57 & 59 & 57 \\
\hline $6-27$ & Mississippi River below Belle Chasse, La. & 5.6 & 5.7 & 4.1 & 1.7 & 2 & 2.2 & 58 & 57 & 62 \\
\hline
\end{tabular}


Table 24.- Concentration data for dissolved trace elements in water samples collected from the Mississippi River and some of its tributaries during the May-June 1990 cruise - Continued

\begin{tabular}{|c|c|c|c|c|c|c|c|c|c|c|}
\hline \multirow{2}{*}{$\begin{array}{l}\text { Date } \\
1990\end{array}$} & \multirow[b]{2}{*}{ Sampling site } & \multicolumn{3}{|c|}{ Beryllium } & \multicolumn{3}{|c|}{ Boron } & \multicolumn{3}{|c|}{ Cadmium } \\
\hline & & $\mathbf{A}$ & B & Grab & A & B & Grab & $\mathbf{A}$ & B & Grab \\
\hline $6-07$ & Illinois River at Valley City, Ill. & $<0.02$ & $<0.02$ & $<0.02$ & 96 & 96 & 29 & $<0.1$ & $<0.1$ & $<0.1$ \\
\hline $6-10$ & Missouri River at St. Charles, Mo. ${ }^{1}$ & 0.032 & - & 0.23 & 34 & - & 36 & $<0.1$ & - & $<0.1$ \\
\hline $6-11$ & Mississippi River below Grafton, Ill. & $<0.02$ & 0.028 & 0.05 & 33 & 33 & 36 & $<0.1$ & $<0.1$ & $<0.1$ \\
\hline $6-13$ & Mississippi River at Thebes, Ill. & 0.03 & 0.028 & 0.1 & 36 & 36 & 35 & $<0.1$ & $<0.1$ & $<0.1$ \\
\hline $6-14$ & Ohio River at Olmsted, Ill. & 0.02 & 0.047 & $<0.02$ & 30 & 29 & 36 & $<0.1$ & $<0.1$ & $<0.1$ \\
\hline $6-18$ & Mississippi River below Memphis, Tenn. & $<0.02$ & $<0.02$ & $<0.02$ & 39 & 41 & 38 & $<0.1$ & $<0.1$ & $<0.1$ \\
\hline $6-19$ & White River at Mile 1.0, Ark. & - & - & $<0.02$ & - & - & 9.2 & - & - & $<0.1$ \\
\hline $6-19$ & Arkansas River at Mile 1.0, Ark. & - & - & $<0.02$ & - & - & 24 & - & - & $<0.1$ \\
\hline $6-20$ & Mississippi River below Arkansas City, Ark. & $<0.02$ & $<0.02$ & $<0.02$ & 35 & 34 & 33 & $<0.1$ & $<0.1$ & $<0.1$ \\
\hline $6-22$ & Yazoo River below Steele Bayou, Miss. & $<0.02$ & $<0.02$ & $<0.02$ & 22 & 26 & 24 & $<0.1$ & $<0.1$ & $<0.1$ \\
\hline $6-23$ & Mississippi River below Vicksburg, Miss. & $<0.02$ & $<0.02$ & 0.044 & 33 & 38 & 36 & $<0.1$ & $<0.1$ & 0.14 \\
\hline $6-25$ & Mississippi River near St. Francisville, La. & $<0.02$ & $<0.02$ & 0.038 & 32 & 32 & 32 & $<0.1$ & $<0.1$ & $<0.1$ \\
\hline $6-27$ & Mississippi River below Belle Chasse, La. & $<0.02$ & 0.009 & $<0.02$ & 26 & 31 & 35 & $<0.1$ & $<0.1$ & $<0.1$ \\
\hline
\end{tabular}

Table 24. - Concentration data for dissolved trace elements in water samples collected from the Mississippi River and some of its tributaries during the May-June 1990 cruise - Continued

\begin{tabular}{|c|c|c|c|c|c|c|c|c|c|c|}
\hline \multirow{2}{*}{$\begin{array}{l}\text { Date } \\
1990\end{array}$} & \multirow[b]{2}{*}{ Sampling site } & \multicolumn{3}{|c|}{ Chromium } & \multicolumn{3}{|c|}{ Cobalt } & \multicolumn{3}{|c|}{ Copper } \\
\hline & & A & B & Grab & A & B & Grab & A & B & Grab \\
\hline $6-07$ & Illinois River at Valley City, Ill. & 0.18 & $<0.2$ & $<0.2$ & 0.61 & 0.7 & 0.39 & 1.5 & 1.5 & 1.5 \\
\hline $6-10$ & Missouri River at St. Charles, Mo. ${ }^{1}$ & $<0.2$ & - & 2.4 & 0.28 & - & 1.2 & 1.4 & - & 3.6 \\
\hline $6-11$ & Mississippi River below Grafton, Ill. & $<0.2$ & $<0.2$ & $<0.2$ & 0.38 & 0.36 & 0.38 & 2.1 & 1.4 & 1.3 \\
\hline $6-13$ & Mississippi River at Thebes, Ill. & $<0.2$ & $<0.2$ & 1.4 & 0.32 & 0.33 & 0.28 & 2.2 & 1.7 & 1.5 \\
\hline $6-14$ & Ohio River at Olmsted, Ill. & $<0.2$ & $<0.2$ & $<0.2$ & 0.31 & 0.45 & 0.26 & 1.5 & 1.5 & 1.3 \\
\hline $6-18$ & Mississippi River below Memphis, Tenn. & $<0.2$ & $<0.2$ & $<0.2$ & 0.19 & 0.27 & 0.3 & 1.6 & 1.6 & 1.6 \\
\hline $6-19$ & White River at Mile 1.0, Ark. & - & - & 0.81 & - & - & 0.2 & - & - & 0.98 \\
\hline $6-19$ & Arkansas River at Mile 1.0, Ark. & - & - & $<0.2$ & - & - & 0.23 & - & - & 1.5 \\
\hline $6-20$ & Mississippi River below Arkansas City, Ark. & 0.22 & 2.5 & 0.41 & 0.17 & 0.63 & 0.3 & 1.8 & 2.1 & 1.6 \\
\hline $6-22$ & Yazoo River below Steele Bayou, Miss. & $<0.2$ & $<0.2$ & $<0.2$ & 0.03 & 0.2 & 0.14 & 1.8 & 1.8 & 1.6 \\
\hline $6-23$ & Mississippi River below Vicksburg, Miss. & $<0.2$ & $<0.2$ & $<0.2$ & 0.32 & 0.35 & 0.33 & 1.7 & 1.7 & 1.6 \\
\hline $6-25$ & Mississippi River near St. Francisville, La. & $<0.2$ & 0.23 & $<0.2$ & 0.30 & 0.37 & 0.31 & 1.9 & 1.9 & 2 \\
\hline $6-27$ & Mississippi River below Belle Chasse, La. & $<0.2$ & $<0.2$ & $<0.2$ & 0.24 & 0.28 & 0.29 & 1.6 & 1.5 & 1.5 \\
\hline
\end{tabular}


Table 24.- Concentration data for dissolved trace elements in water samples collected from the Mississippi River and some of its tributaries during the May-June 1990 cruise - Continued

\begin{tabular}{|c|c|c|c|c|c|c|c|c|c|c|}
\hline \multirow{2}{*}{$\begin{array}{l}\text { Date } \\
1990\end{array}$} & \multirow[b]{2}{*}{ Sampling site } & \multicolumn{3}{|c|}{ Iron } & \multicolumn{3}{|c|}{ Lead } & \multicolumn{3}{|c|}{ Lithium } \\
\hline & & $\bar{A}$ & B & Grab & $\mathbf{A}$ & B & Grab & $\mathbf{A}$ & $\mathbf{B}$ & Grab \\
\hline$\overline{6-07}$ & Illinois River at Valley City, III. & - & 0.008 & $<0.005$ & 0.08 & 0.11 & $<0.06$ & $\overline{4.3}$ & $\overline{4.3}$ & 3.8 \\
\hline $6-10$ & Missouri River at St. Charles, Mo. ${ }^{1}$ & 0.034 & - & 2.3 & $<0.06$ & - & 2.1 & 8.3 & - & 10 \\
\hline $6-11$ & Mississippi River below Grafton, Ill. & - & $<0.005$ & 0.009 & $<0.06$ & $<0.06$ & $<0.06$ & 3.7 & 3.2 & 3.5 \\
\hline $6-13$ & Mississippi River at Thebes, Ill. & - & 0.029 & 0.007 & $<0.06$ & $<0.06$ & $<0.06$ & 5.1 & 5.1 & 4.9 \\
\hline $6-14$ & Ohio River at Olmsted, Ill. & - & $<0.005$ & $<0.005$ & $<0.06$ & $<0.06$ & $<0.06$ & 1.8 & 1.8 & 2.2 \\
\hline 6-18 & Mississippi River below Memphis, Tenn. & -- & 0.009 & 0.005 & $<0.06$ & $<0.06$ & $<0.06$ & 3.7 & 3.8 & 3.5 \\
\hline $6-19$ & White River at Mile 1.0, Ark. & - & - & $<0.005$ & - & - & $<0.06$ & - & - & 0.31 \\
\hline 6-19 & Arkansas River at Mile 1.0, Ark. & - & - & $<0.005$ & - & - & $<0.06$ & - & - & 1.7 \\
\hline $6-20$ & Mississippi River below Arkansas City, Ark. & - & 0.65 & $<0.005$ & $<0.06$ & 0.62 & $<0.06$ & 3.2 & 3.8 & 3.3 \\
\hline $6-22$ & Yazoo River below Steele Bayou, Miss. & - & 0.011 & 0.006 & $<0.06$ & $<0.06$ & $<0.06$ & 1.5 & 1.7 & 1.6 \\
\hline $6-23$ & Mississippi River below Vicksburg, Miss. & - & $<0.005$ & $<0.005$ & $<0.06$ & $<0.06$ & $<0.06$ & 2.9 & 3.4 & 3 \\
\hline $6-25$ & Mississippi River near St. Francisville, La. & - & 0.094 & $<0.005$ & $<0.06$ & 0.12 & $<0.06$ & 2.9 & 3.2 & 3.1 \\
\hline 6-27 & Mississippi River below Belle Chasse, La. & - & $<0.005$ & $<0.005$ & $<0.06$ & $<0.06$ & $<0.06$ & 2.8 & 3.3 & 3.3 \\
\hline
\end{tabular}

Table 24.-- Concentration data for dissolved trace elements in water samples collected from the Mississippi River and some of its tributaries during the May-June 1990 cruise - Continued

\begin{tabular}{|c|c|c|c|c|c|c|c|c|c|c|}
\hline \multirow{2}{*}{$\begin{array}{l}\text { Date } \\
1990 \\
\end{array}$} & \multirow[b]{2}{*}{ Sampling site } & \multicolumn{3}{|c|}{ Manganese } & \multicolumn{3}{|c|}{ Molybdenum } & \multicolumn{3}{|c|}{ Nickel } \\
\hline & & $\bar{A}$ & B & Grab & $\bar{A}$ & B & Grab & A & $\mathbf{B}$ & Grab \\
\hline $6-07$ & Illinois River at Valley City, Ill. & 3.0 & 8 & 0.41 & 3.7 & 3.6 & 1.4 & - & 14 & 8.4 \\
\hline $6-10$ & Missouri River at St. Charles, Mo. ${ }^{1}$ & 1.9 & - & 65 & 1.9 & - & 0.65 & 6.2 & - & 9.5 \\
\hline $6-11$ & Mississippi River below Grafton, Ill. & 0.41 & 0.49 & 1 & 1.9 & 1.9 & 1.7 & - & 8.4 & 8.8 \\
\hline $6-13$ & Mississippi River at Thebes, Ill. & 0.36 & 0.7 & 0.52 & 1.7 & 1.7 & 1.8 & - & 7.4 & 7.3 \\
\hline 6-14 & Ohio River at Olmsted, Ill. & 0.19 & 0.47 & 0.64 & 1.5 & 1.5 & 1.4 & - & 6.4 & 6.5 \\
\hline $6-18$ & Mississippi River below Memphis, Tenn. & 0.30 & 0.75 & 0.57 & 1.7 & 1.7 & 1.7 & -- & 6.6 & 6.6 \\
\hline 6-19 & White River at Mile 1.0, Ark. & - & - & 28 & - & - & 0.67 & - & - & 4.6 \\
\hline $6-19$ & Arkansas River at Mile 1.0, Ark. & - & -- & 0.93 & - & - & 0.56 & - & - & 6.2 \\
\hline $6-20$ & Mississippi River below Arkansas City, Ark. & 0.91 & 34 & 0.55 & 1.0 & 0.93 & 1.6 & - & 7 & 6.6 \\
\hline $6-22$ & Yazoo River below Steele Bayou, Miss. & 58 & 68 & 80 & 0.61 & 0.65 & 0.48 & - & 3.1 & 3.3 \\
\hline $6-23$ & Mississippi River below Vicksburg, Miss. & 0.67 & 0.67 & 2.9 & 1.5 & 1.5 & 1.7 & - & 6.1 & 6.1 \\
\hline $6-25$ & Mississippi River near St. Francisville, La. & 3.1 & 9.3 & 1.5 & 1.5 & 1.5 & 1.5 & - & 6.7 & 6.6 \\
\hline $6-27$ & Mississippi River below Belle Chasse, La. & 0.76 & 0.74 & 1.2 & 1.5 & 1.7 & 1.6 & -- & 6.5 & 6.4 \\
\hline
\end{tabular}


Table 24.-- Concentration data for dissolved trace elements in water samples collected from the Mississippi River and some of its tributaries during the May-June 1990 cruise - Continued

\begin{tabular}{|c|c|c|c|c|c|c|c|c|c|c|}
\hline \multirow{2}{*}{$\begin{array}{l}\text { Date } \\
1990\end{array}$} & \multirow[b]{2}{*}{ Sampling site } & \multicolumn{3}{|c|}{ Strontium } & \multicolumn{3}{|c|}{ Thallium } & \multicolumn{3}{|c|}{ Uranium } \\
\hline & & A & B & Grab & A & B & Grab & A & B & Grab \\
\hline $6-07$ & Illinois River at Valley City, Ill. & 0.22 & 0.21 & 0.11 & $<0.05$ & $<0.05$ & $<0.05$ & 1.8 & 1.8 & 1.6 \\
\hline $6-10$ & Missouri River at St. Charles, Mo.' & 0.19 & - & 0.2 & $<0.05$ & - & $<0.05$ & 1.8 & - & 1.8 \\
\hline $6-11$ & Mississippi River below Grafton, IIl. & 0.14 & 0.12 & 0.12 & $<0.05$ & $<0.05$ & $<0.05$ & 1.8 & 2.1 & 1.7 \\
\hline $6-13$ & Mississippi River at Thebes, Ill. & 0.16 & 0.16 & 0.16 & $<0.05$ & $<0.05$ & $<0.05$ & 1.5 & 1.6 & 1.3 \\
\hline $6-14$ & Ohio River at Olmsted, Ill. & 0.14 & 0.12 & 0.13 & $<0.05$ & $<0.05$ & $<0.05$ & 0.24 & 0.18 & 0.24 \\
\hline $6-18$ & Mississippi River below Memphis, Tenn. & 0.16 & 0.15 & 0.15 & $<0.05$ & $<0.05$ & $<0.05$ & 0.83 & 0.83 & 0.91 \\
\hline $6-19$ & White River at Mile 1.0, Ark. & - & -- & 0.041 & - & - & $<0.05$ & - & - & 0.17 \\
\hline $6-19$ & Arkansas River at Mile 1.0, Ark. & - & - & 0.16 & - & - & $<0.05$ & - & - & 0.52 \\
\hline $6-20$ & Mississippi River below Arkansas City, Ark. & 0.15 & 0.14 & 0.14 & $<0.05$ & 0.051 & $<0.05$ & 0.81 & 0.81 & 0.64 \\
\hline $6-22$ & Yazoo River below Steele Bayou, Miss. & 0.059 & 0.057 & 0.058 & $<0.05$ & $<0.05$ & $<0.05$ & $<0.06$ & 0.11 & 0.074 \\
\hline $6-23$ & Mississippi River below Vicksburg, Miss. & 0.15 & 0.14 & 0.14 & $<0.05$ & 0.051 & $<0.05$ & 0.83 & 0.69 & 0.66 \\
\hline $6-25$ & Mississippi River near St. Francisville, La. & 0.15 & 0.14 & 0.14 & $<0.05$ & $<0.05$ & $<0.05$ & 0.79 & 0.86 & 0.73 \\
\hline $6-27$ & Mississippi River below Belle Chasse, La. & 0.15 & 0.15 & 0.15 & $<0.05$ & $<0.05$ & $<0.05$ & 0.64 & 0.67 & 0.6 \\
\hline
\end{tabular}

Table 24.- Concentration data for dissolved trace elements in water samples collected from the Mississippi River and some of its tributaries during the May-June 1990 cruise - Continued

\begin{tabular}{|c|c|c|c|c|c|c|c|}
\hline \multirow{2}{*}{$\begin{array}{l}\text { Date } \\
1990\end{array}$} & \multirow[b]{2}{*}{ Sampling site } & \multicolumn{3}{|c|}{ Vanadium } & \multicolumn{3}{|c|}{ Zinc } \\
\hline & & A & B & Grab & A & B & Grab \\
\hline $6-07$ & Illinois River at Valley City, IIl. & 1.9 & 1.8 & 1.8 & 2.2 & 2.2 & 0.73 \\
\hline $6-10$ & Missouri River at St. Charles, Mo. ${ }^{1}$ & 2.1 & - & 6.2 & 1.1 & - & 9.7 \\
\hline 6-11 & Mississippi River below Grafton, Ill. & 1.7 & 1.7 & 1.9 & 0.94 & 0.81 & 0.92 \\
\hline $6-13$ & Mississippi River at Thebes, Ill. & 2.4 & 2.1 & 1.9 & 0.88 & 1.6 & 0.58 \\
\hline $6-14$ & Ohio River at Olmsted, Ill. & 0.63 & 0.63 & 0.55 & 0.87 & 1.9 & 0.71 \\
\hline $6-18$ & Mississippi River below Memphis, Tenn. & 1.5 & 1.5 & 1.5 & 0.57 & 0.56 & 0.76 \\
\hline $6-19$ & White River at Mile 1.0, Ark. & - & - & 0.66 & - & -- & 0.96 \\
\hline $6-19$ & Arkansas River at Mile 1.0, Ark. & - & -- & 1.2 & - & -- & 0.96 \\
\hline $6-20$ & Mississippi River below Arkansas City, Ark. & 1.5 & 2.7 & 1.6 & 0.91 & 3.8 & 0.7 \\
\hline $6-22$ & Yazoo River below Steele Bayou, Miss. & 1.0 & 1.1 & 1 & 0.73 & 0.73 & 1.2 \\
\hline $6-23$ & Mississippi River below Vicksburg, Miss. & 1.5 & 1.5 & 1.4 & 0.63 & 0.63 & 0.79 \\
\hline $6-25$ & Mississippi River near St. Francisville, La. & 1.5 & 1.7 & 1.5 & 0.93 & 1.7 & 0.73 \\
\hline $6-27$ & Mississippi River below Belle Chasse, La. & 1.5 & 1.6 & 1.9 & 1.2 & 0.73 & 1.3 \\
\hline
\end{tabular}

'Single vertical composite near centroid of flow 


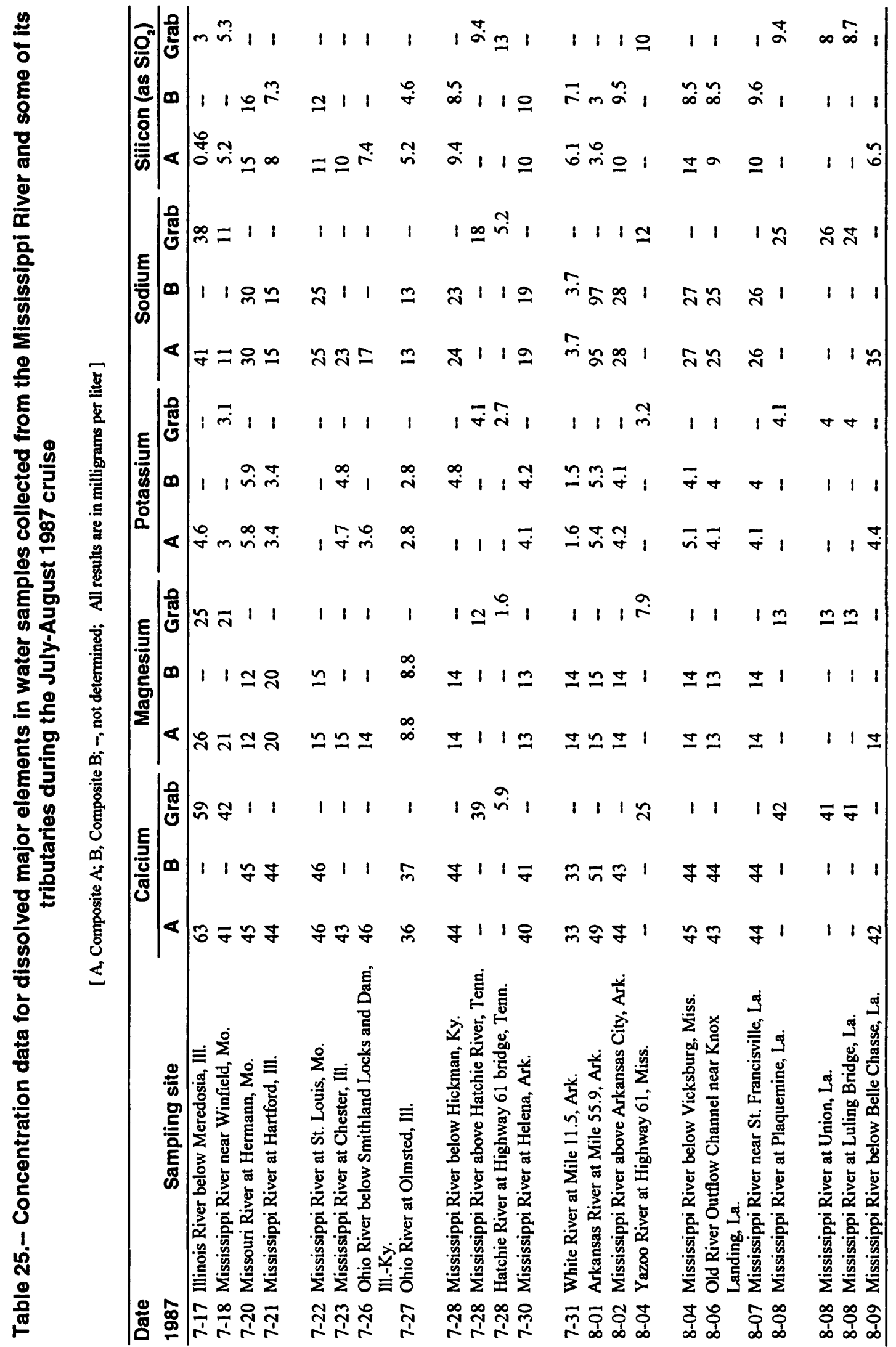



Table 26.- Concentration data for dissolved major elements in water samples collected from the
Mississippi River and some of its tributaries during the November-December 1987 cruise

[ A, Composite A; B, Composite B; -, not determined; All results are in milligrams per liter ]

\begin{tabular}{|c|c|c|c|c|c|c|c|c|c|}
\hline \multirow[b]{2}{*}{ Sampling site } & \multicolumn{3}{|c|}{ Calcium } & \multicolumn{3}{|c|}{ Magnesium } & \multicolumn{3}{|c|}{ Potassium } \\
\hline & A & B & Grab & A & B & Grab & A & B & Grab \\
\hline 11-29 Illinois River below Meredosia, IIl. & 64 & - & -- & 29 & - & - & 4.5 & - & - \\
\hline 11-30 Mississippi River near Winfield, Mo. & 40 & 43 & - & 21 & 22 & - & 2.7 & 2.8 & - \\
\hline 12-02 Missouri River at St. Charles, Mo. & 56 & 55 & - & 19 & 19 & -- & 4.7 & 4.7 & - \\
\hline 12-03 Mississippi River at St. Louis, Mo. & 50 & 47 & -- & 20 & 19 & - & 4.6 & 3.8 & - \\
\hline 12-05 Mississippi River at Thebes, Ill. & 46 & 48 & - & 19 & 20 & - & 3.9 & 3.9 & - \\
\hline 12-06 Ohio River at Olmsted, Ill. & 38 & 38 & - & 11 & 11 & - & 3.3 & 3.2 & - \\
\hline 12-07 Mississippi River below Hickman, Ky. & 44 & 44 & - & 16 & 16 & - & 3.6 & 3.5 & -- \\
\hline 12-08 Mississippi River at Fulton, Tenn. & 44 & 44 & - & 16 & 16 & - & 3.5 & 3.6 & -- \\
\hline 12-11 Mississippi River at Helena, Ark. & 42 & 43 & -- & 15 & 15 & -- & 3.5 & 3.5 & - \\
\hline 12-12 White River at Mile 11.5, Ark. & 23 & 23 & -- & 8.8 & 8.8 & - & 2.4 & 2.4 & - \\
\hline 12-13 Mississippi River above Arkansas City, Ark. & 40 & 41 & - & 15 & 15 & - & 3.5 & 3.5 & - \\
\hline 12-14 Yazoo River at Mile 10, Miss. & 8 & 8.1 & - & 2.8 & 2.8 & - & 3.3 & 3.3 & -- \\
\hline 12-15 Mississippi River below Vicksburg, Miss. & 38 & 38 & - & 14 & 14 & -- & 3.5 & 3.5 & - \\
\hline $\begin{array}{l}\text { 12-17 Old River Outflow Channel near Knox } \\
\text { Landing, La. }\end{array}$ & 39 & 39 & - & 14 & 14 & - & 3.7 & 3.5 & - \\
\hline 12-18 Mississippi River near St. Francisville, La. & 40 & 40 & - & 14 & 14 & - & 3.5 & 3.5 & -- \\
\hline 12-20 Mississippi River below Belle Chasse, La. & 36 & 37 & -- & 14 & 14 & - & 3.7 & 3.7 & -- \\
\hline
\end{tabular}

Table 26. - Concentration data for dissolved major elements in water samples collected from the Mississippi River and some of its tributaries during the November-December 1987 cruise Continued

\begin{tabular}{|c|c|c|c|c|c|c|}
\hline \multirow{2}{*}{$\begin{array}{l}\text { Date } \\
1987\end{array}$} & \multicolumn{3}{|c|}{ Sodium } & \multicolumn{3}{|c|}{ Silicon (as $\mathrm{SiO}_{2}$ ) } \\
\hline & $\mathbf{A}$ & B & Grab & $\mathbf{A}$ & B & Grab \\
\hline 11-29 Illinois River below Meredosia, Ill. & 44 & - & - & 3.3 & -- & -- \\
\hline 11-30 Mississippi River near Winfield, Mo. & 12 & 13 & - & 1.3 & 0.92 & - \\
\hline 12-02 Missouri River at St. Charles, Mo. & 44 & 43 & - & 12 & 11 & - \\
\hline 12-03 Mississippi River at St. Louis, Mo. & 28 & 26 & - & 7.6 & 8.1 & - \\
\hline 12-05 Mississippi River at Thebes, Ill. & 25 & 27 & -- & 9.9 & 7.5 & -- \\
\hline 12-06 Ohio River at Olmsted, Ill. & 24 & 23 & - & 5.6 & 6.4 & - \\
\hline 12-07 Mississippi River below Hickman, Ky. & 25 & 25 & - & 7 & 6.2 & - \\
\hline 12-08 Mississippi River at Fulton, Tenn. & 26 & 26 & - & 7.4 & 6.4 & - \\
\hline 12-11 Mississippi River at Helena, Ark. & 25 & 25 & - & 6.1 & 6.9 & -- \\
\hline 12-12 White River at Mile 11.5, Ark. & 3.7 & 3.7 & - & 9.8 & 9 & - \\
\hline 12-13 Mississippi River above Arkansas City, Ark. & 28 & 28 & - & 5.3 & 5.2 & -- \\
\hline 12-14 Yazoo River at Mile 10, Miss. & 5.7 & 5.7 & - & 11 & 10 & - \\
\hline 12-15 Mississippi River below Vicksburg, Miss. & 25 & 26 & -- & 5.7 & 6 & -- \\
\hline $\begin{array}{l}\text { 12-17 Old River Outflow Channel near Knox } \\
\text { Landing, La. }\end{array}$ & 26 & 27 & -- & 5.9 & 7.3 & -- \\
\hline 12-18 Mississippi River near St. Francisville, La. & 27 & 27 & - & 7.8 & 6.8 & - \\
\hline 12-20 Mississippi River below Belle Chasse, La. & 33 & 34 & - & 6.4 & 5.9 & - \\
\hline
\end{tabular}




\section{Table 27. - Concentration data for dissolved major elements in water samples collected from the Mississippi River and some of its tributaries during the May-June 1988 cruise}

[ A, Composite A; B, Composite B; --, not determined; <, less than detection limit; All results are in milligrams per liter ]

\begin{tabular}{|c|c|c|c|c|c|c|c|c|c|c|}
\hline \multirow{2}{*}{$\begin{array}{l}\text { Date } \\
1988\end{array}$} & \multirow[b]{2}{*}{ Sampling site } & \multicolumn{3}{|c|}{ Calcium } & \multicolumn{3}{|c|}{ Magnesium } & \multicolumn{3}{|c|}{ Potassium } \\
\hline & & A & B & Grab & A & B & Grab & A & B & Grab \\
\hline $5-16$ & Illinois River below Meredosia, Ill. & 61 & $\overline{62}$ & 63 & 30 & 30 & 31 & 4 & 4.1 & 4.1 \\
\hline $5-17$ & Mississippi River near Winfield, Mo. & 34 & 39 & 38 & 21 & 23 & 22 & 3.3 & 3.4 & 3.3 \\
\hline $5-19$ & Missouri River at Hermann, Mo. & 63 & 63 & 57 & 21 & 21 & 19 & 7.4 & 7.3 & 7.2 \\
\hline $5-20$ & Mississippi River at St. Louis, Mo. & 52 & 52 & 43 & 23 & 23 & 22 & 5.6 & 4.6 & 4 \\
\hline $5-22$ & Mississippi River at Thebes, Ill. & 54 & 53 & 50 & 24 & 23 & 22 & 4.6 & 4.6 & 4.6 \\
\hline $5-23$ & Ohio River at Olmsted, Ill. & 37 & 36 & 37 & 12 & 12 & 13 & 3 & 3 & 3 \\
\hline $5-24$ & Mississippi River below Hickman, Ky. & 46 & 46 & 50 & 18 & 18 & 21 & 3.9 & 3.9 & 4.3 \\
\hline $5-26$ & Mississippi River at Fulton, Tenn. & 45 & 42 & 44 & 17 & 17 & 17 & 3.9 & 3.9 & 3.9 \\
\hline $5-28$ & Mississippi River at Helena, Ark. & 43 & 42 & 43 & 16 & 16 & 17 & 4 & 3.9 & 4 \\
\hline $5-29$ & White River at Mile 11.5, Ark. & 33 & 33 & 33 & 12 & 12 & 12 & 3.9 & 2.1 & 2.1 \\
\hline $5-29$ & Arkansas River at Mile 1.0, Ark. & - & - & 48 & - & - & 12 & - & - & 4.1 \\
\hline $5-30$ & Mississippi River above Arkansas City, Ark. & 42 & 42 & 43 & 16 & 16 & 16 & 3.8 & 3.8 & 3.8 \\
\hline 6-01 & Yazoo River at Mile 10, Miss. & 25 & - & 25 & 7.9 & - & 7.9 & 3.4 & - & 3.4 \\
\hline 6-02 & Mississippi River below Vicksburg, Miss. & 45 & 45 & 44 & 17 & 17 & 17 & 3.8 & 3.8 & 3.8 \\
\hline $6-04$ & $\begin{array}{l}\text { Old River Outflow Channel near Knox } \\
\text { Landing, La. }\end{array}$ & 43 & 46 & - & 16 & 17 & - & 3.8 & 3.8 & - \\
\hline 6-05 & Mississippi River near St. Francisville, La. & 46 & 47 & 47 & 17 & 17 & 17 & 3.8 & 3.8 & 3.9 \\
\hline 6-07 & Mississippi River below Belle Chasse, La. & 50 & 45 & 46 & 18 & 17 & 17 & 4.1 & 4.1 & 4.1 \\
\hline
\end{tabular}

Table 27. - Concentration data for dissolved major elements in water samples collected from the Mississippi River and some of its tributaries during the May-June 1988 cruise - Continued

\begin{tabular}{|c|c|c|c|c|c|c|c|}
\hline \multirow{2}{*}{$\begin{array}{l}\text { Date } \\
1988\end{array}$} & \multirow[b]{2}{*}{ Sampling site } & \multicolumn{3}{|c|}{ Sodium } & \multicolumn{3}{|c|}{ Silicon (as $\mathrm{SiO}_{2}$ ) } \\
\hline & & A & B & Grab & A & B & Grab \\
\hline $5-16$ & Illinois River below Meredosia, Ill. & 34 & 34 & 35 & 0.42 & 1.1 & 2.6 \\
\hline $5-17$ & Mississippi River near Winfield, Mo. & 11 & 12 & 12 & 0.31 & - & 0.13 \\
\hline $5-19$ & Missouri River at Hermann, Mo. & 54 & 54 & 47 & 9.1 & 10 & 8.4 \\
\hline $5-20$ & Mississippi River at St. Louis, Mo. & 32 & 32 & 21 & 3.9 & 4.2 & 1.4 \\
\hline $5-22$ & Mississippi River at Thebes, Ill. & 34 & 33 & 31 & 3.9 & 3.9 & 3.6 \\
\hline $5-23$ & Ohio River at Olmsted, Ill. & 17 & 17 & 18 & 0.34 & 0.33 & 0.2 \\
\hline $5-24$ & Mississippi River below Hickman, Ky. & 26 & 26 & 29 & 2.3 & 2.3 & 3.1 \\
\hline $5-26$ & Mississippi River at Fulton, Tenn. & 24 & 23 & 24 & 2.1 & 2 & 2 \\
\hline $5-28$ & Mississippi River at Helena, Ark. & 22 & 22 & 23 & 2.9 & 2 & 3.5 \\
\hline $5-29$ & White River at Mile 11.5, Ark. & 3.5 & 3.6 & 3.6 & 3.9 & 3.9 & 3.8 \\
\hline $5-29$ & Arkansas River at Mile 1.0, Ark. & - & - & 62 & - & - & 3.7 \\
\hline $5-30$ & Mississippi River above Arkansas City, Ark. & 22 & 22 & 22 & 2 & 2 & 1.9 \\
\hline 6-01 & Yazoo River at Mile 10, Miss. & 13 & - & 13 & 10 & - & 10 \\
\hline 6-02 & Mississippi River below Vicksburg, Miss. & 23 & 23 & 23 & 1.7 & 1.7 & 1.8 \\
\hline $6-04$ & $\begin{array}{l}\text { Old River Outflow Channel near Knox } \\
\text { Landing, La. }\end{array}$ & 22 & 24 & $<0.01$ & 1.9 & 1.9 & $<0.04$ \\
\hline $6-05$ & Mississippi River near St. Francisville, La. & 24 & 24 & 24 & 1.9 & 1.9 & 1.9 \\
\hline $6-07$ & Mississippi River below Belle Chasse, La. & 34 & 30 & 31 & 2.5 & 1.7 & 1.6 \\
\hline
\end{tabular}


Table 28. - Concentration data for dissolved major elements in water samples collected from the Mississippi River and some of its tributaries during the March-April 1989 cruise

[ A, Composite A; B, Composite B; --, not determined; All results are in milligrams per liter ]

\begin{tabular}{|c|c|c|c|c|c|c|c|c|c|c|}
\hline \multirow{2}{*}{$\begin{array}{l}\text { Date } \\
1989\end{array}$} & \multirow[b]{2}{*}{ Sampling site } & \multicolumn{3}{|c|}{ Calcium } & \multicolumn{3}{|c|}{ Magnesium } & \multicolumn{3}{|c|}{ Potassium } \\
\hline & & A & B & Grab & $\bar{A}$ & B & Grab & $\mathbf{A}$ & B & Grab \\
\hline $3-09$ & Illinois River at Hardin, Ill. & 79 & 80 & 79 & 31 & 31 & 31 & 4.2 & $\overline{4.2}$ & 4.1 \\
\hline $3-10$ & Mississippi River near Winfield, Mo. & 50 & 50 & 50 & 21 & 21 & 21 & 3.5 & 3.4 & 3.2 \\
\hline $3-12$ & Missouri River at Hermann, Mo. & 45 & 44 & 42 & 16 & 16 & 16 & 3.7 & 3.5 & 3.5 \\
\hline 3-13 & Mississippi River at St. Louis, Mo. & 52 & 52 & - & 20 & 20 & - & 3.7 & 3.9 & - \\
\hline $3-15$ & Mississippi River at Thebes, IIl. & 48 & 48 & 48 & 18 & 19 & 19 & 3.7 & 3.8 & 3.6 \\
\hline $3-16$ & Ohio River at Olmsted, Ill. & 28 & 27 & 28 & 6.3 & 6.3 & 6 & 1.7 & 1.7 & 1.7 \\
\hline 3-17 & Mississippi River below Hickman, Ky. & 31 & 32 & 31 & 8.5 & 8.8 & 8.4 & 2.3 & 2.2 & 1.9 \\
\hline 3-19 & Mississippi River below Fulton, Tenn. & 31 & 31 & 31 & 8.5 & 8.6 & 8.6 & 2.1 & 2.2 & 2.2 \\
\hline $3-21$ & Mississippi River at Helena, Ark. & 32 & 31 & 31 & 8.7 & 8.6 & 8.4 & 2.1 & 2.2 & 1.9 \\
\hline $3-22$ & White River at Mile 11.5, Ark. & 18 & 18 & 18 & 6.7 & 6.7 & 6.8 & 1.5 & 1.5 & 1.5 \\
\hline $3-23$ & Arkansas River at Pendleton, Ark. & 24 & 24 & 26 & 6.2 & 6.2 & 6.6 & 2.2 & 2.2 & 2.1 \\
\hline $3-24$ & Mississippi River above Arkansas City, Ark. & 33 & 31 & 31 & 9.4 & 9.1 & 9.1 & 2.3 & 2.2 & 2.3 \\
\hline $3-26$ & Yazoo River below Steele Bayou, Miss. & 7.1 & 7.1 & 7.7 & 2.2 & 2.2 & 2.3 & 2.4 & 2.6 & 2.7 \\
\hline 3-27 & Mississippi River below Vicksburg, Miss. & 32 & 32 & 33 & 9 & 9 & 9.4 & 2.3 & 2.3 & 2.3 \\
\hline 3-29 & $\begin{array}{l}\text { Old River Outflow Channel near Knox } \\
\text { Landing, La. }\end{array}$ & 32 & 32 & 32 & 8.8 & 8.8 & 8.9 & 2.4 & 2.2 & 2.3 \\
\hline $3-30$ & Mississippi River near St. Francisville, La. & 31 & 31 & 31 & 8.7 & 8.8 & 8.8 & 2.3 & 2.4 & 2.3 \\
\hline 4-01 & Mississippi River below Belle Chasse, La. & 31 & 31 & 31 & 8.6 & 8.6 & 8.6 & 2.4 & 2.3 & 2.3 \\
\hline
\end{tabular}

Table 28. - Concentration data for dissolved major elements in water samples collected from the Mississippi River and some of its tributaries during the March-April 1989 cruise - Continued

\begin{tabular}{|c|c|c|c|c|c|c|c|}
\hline \multirow{2}{*}{$\begin{array}{l}\text { Date } \\
1989\end{array}$} & \multirow[b]{2}{*}{ Sampling site } & \multicolumn{3}{|c|}{ Sodium } & \multicolumn{3}{|c|}{ Silicon (as $\mathrm{SiO}_{2}$ ) } \\
\hline & & A & B & Grab & $\bar{A}$ & B & Grab \\
\hline $3-09$ & Illinois River at Hardin, Ill. & 73 & 73 & 72 & 5.4 & 5.1 & 5.1 \\
\hline 3-10 & Mississippi River near Winfield, Mo. & 18 & 18 & 18 & 4.7 & 4.6 & 4.3 \\
\hline $3-12$ & Missouri River at Hermann, Mo. & 33 & 32 & 28 & 9.3 & 9.1 & 8.3 \\
\hline $3-13$ & Mississippi River at St. Louis, Mo. & 36 & 36 & - & 5.7 & 5.7 & - \\
\hline $3-15$ & Mississippi River at Thebes, Ill. & 31 & 32 & 32 & 5.7 & 5.7 & 5.5 \\
\hline $3-16$ & Ohio River at Olmsted, Ill. & 7.4 & 7.4 & 6.8 & 5.4 & 5.4 & 5.4 \\
\hline 3-17 & Mississippi River below Hickman, Ky. & 12 & 12 & 11 & 5.3 & 5.2 & 5.3 \\
\hline $3-19$ & Mississippi River below Fulton, Tenn. & 12 & 12 & 12 & 5.2 & 5.2 & 5.2 \\
\hline 3-21 & Mississippi River at Helena, Ark. & 12 & 12 & 11 & 5.5 & 5.8 & 6.1 \\
\hline $3-22$ & White River at Mile 11.5, Ark. & 1.8 & 1.8 & 1.8 & 4.4 & 4.4 & 4.4 \\
\hline 3-23 & Arkansas River at Pendleton, Ark. & 31 & 31 & 34 & 3.8 & 3.8 & 3.8 \\
\hline 3-24 & Mississippi River above Arkansas City, Ark. & 14 & 13 & 14 & 5.6 & 5.4 & 5.3 \\
\hline 3-26 & Yazoo River below Steele Bayou, Miss. & 3.2 & 3.2 & 3.2 & 5.2 & 5.2 & 5.2 \\
\hline 3-27 & Mississippi River below Vicksburg, Miss. & 14 & 14 & 14 & 5.5 & 5.5 & 5.5 \\
\hline $3-29$ & $\begin{array}{l}\text { Old River Outflow Channel near Knox } \\
\text { Landing, La. }\end{array}$ & 14 & 14 & 14 & 5.5 & 5.5 & 5.5 \\
\hline 3-30 & Mississippi River near St. Francisville, La. & 13 & 14 & 14 & 5.4 & 5.4 & 5.4 \\
\hline 4-01 & Mississippi River below Belle Chasse, La. & 15 & 15 & 15 & 5.4 & 5.4 & 5.4 \\
\hline
\end{tabular}




\section{Table 29.- Concentration data for dissolved major elements in water samples collected from the Mississippi River and some of its tributaries during the June 1989 cruise}

[ A, Composite A; B, Composite B; --, not determined; All results are in milligrams per liter ]

\begin{tabular}{|c|c|c|c|c|c|c|c|c|c|c|}
\hline \multirow{2}{*}{$\begin{array}{l}\text { Date } \\
1989\end{array}$} & \multirow[b]{2}{*}{ Sampling site } & \multicolumn{3}{|c|}{ Calcium } & \multicolumn{3}{|c|}{ Magnesium } & \multicolumn{3}{|c|}{ Potassium } \\
\hline & & A & B & Grab & $\bar{A}$ & B & Grab & A & B & Grab \\
\hline $6-04$ & Illinois River at Hardin, Ill. & 70 & - & 70 & 32 & - & 33 & 4.1 & -- & 4.1 \\
\hline 6-05 & Mississippi River near Winfield, Mo. & 39 & 38 & 37 & 19 & 19 & 19 & 3.3 & 3.2 & 3.3 \\
\hline 6-07 & Missouri River at Hermann, Mo. & 53 & 53 & 53 & 23 & 20 & 20 & 5.1 & 4.9 & 4.9 \\
\hline $6-08$ & Mississippi River at St. Louis, Mo. & 49 & 49 & 47 & 20 & 20 & 20 & 4.1 & 4.2 & 3.6 \\
\hline $6-10$ & Mississippi River at Thebes, Ill. & 48 & 48 & 49 & 20 & 20 & 20 & 4.1 & 4.1 & 4 \\
\hline $6-11$ & Ohio River at Olmsted, Ill. & 40 & 40 & 40 & 11 & 11 & 11 & 2.2 & 2.2 & 2.2 \\
\hline $6-12$ & Mississippi River below Hickman, Ky. & 40 & 40 & 40 & 14 & 14 & 14 & 2.8 & 3 & 2.9 \\
\hline 6-14 & Mississippi River at Fulton, Tenn. & 40 & 41 & 41 & 14 & 14 & 14 & 2.9 & 3.1 & 3 \\
\hline 6-17 & Mississippi River at Helena, Ark. & 38 & 40 & 43 & 13 & 15 & 16 & 2.9 & 2.9 & 2.9 \\
\hline $6-18$ & White River at Mile 11.5, Ark. & 30 & - & 31 & 15 & - & 15 & 1.8 & - & 1.7 \\
\hline $6-19$ & Arkansas River at Pendleton, Ark. & 34 & - & 35 & 9.4 & -- & 9.6 & 3 & - & 3 \\
\hline $6-20$ & Mississippi River above Arkansas City, Ark. & 34 & 34 & 34 & 12 & 12 & 12 & 2.6 & 2.7 & 2.8 \\
\hline $6-22$ & Yazoo River below Steele Bayou, Miss. & 5.5 & 5.7 & 5.6 & 1.8 & 1.8 & 1.8 & 2.6 & 2.5 & 2.5 \\
\hline $6-23$ & Mississippi River below Vicksburg, Miss. & 33 & 34 & 34 & 9.6 & 11 & 9.9 & 2.7 & 2.6 & 2.5 \\
\hline $6-25$ & $\begin{array}{l}\text { Old River Outflow Channel near Knox } \\
\text { Landing, La. }\end{array}$ & 32 & 32 & 31 & 9.5 & 9.4 & 9.2 & 2.5 & 2.5 & 2.5 \\
\hline $6-26$ & Mississippi River near St. Francisville, La. & 32 & 34 & 33 & 9.4 & 9.8 & 9.7 & 2.6 & 2.7 & 2.6 \\
\hline $6-28$ & Mississippi River below Belle Chasse, La. & 30 & 28 & 32 & 9.1 & 8.4 & 9.6 & 2.5 & 2.7 & 2.7 \\
\hline
\end{tabular}

Table 29.- Concentration data for dissolved major elements in water samples collected from the Mississippi River and some of its tributaries during the June 1989 cruise - Continued

\begin{tabular}{|c|c|c|c|c|c|c|c|}
\hline \multirow{2}{*}{$\begin{array}{l}\text { Date } \\
1989\end{array}$} & \multirow[b]{2}{*}{ Sampling site } & \multicolumn{3}{|c|}{ Sodium } & \multicolumn{3}{|c|}{ Silicon (as SiO) } \\
\hline & & A & B & Grab & $\bar{A}$ & B & Grab \\
\hline $6-04$ & Illinois River at Hardin, Ill. & 41 & $\overline{-}$ & 41 & 3.3 & - & $\overline{3.3}$ \\
\hline $6-05$ & Mississippi River near Winfield, Mo. & 13 & 13 & 12 & 1.3 & 1.3 & 1.3 \\
\hline $6-07$ & Missouri River at Hermann, Mo. & 51 & 52 & 51 & 7.9 & 7.7 & 7.7 \\
\hline $6-08$ & Mississippi River at St. Louis, Mo. & 33 & 33 & 29 & 4.6 & 4.5 & 3.9 \\
\hline $6-10$ & Mississippi River at Thebes, Ill. & 32 & 32 & 32 & 4.3 & 4.3 & 4.3 \\
\hline $6-11$ & Ohio River at Olmsted, Ill. & 9.4 & 9.5 & 9.5 & 5.4 & 5.5 & 5.5 \\
\hline $6-12$ & Mississippi River below Hickman, Ky. & 16 & 17 & 15 & 4.7 & 4.8 & 4.9 \\
\hline $6-14$ & Mississippi River at Fulton, Tenn. & 17 & 17 & 17 & 4.9 & 4.9 & 4.9 \\
\hline $6-17$ & Mississippi River at Helena, Ark. & 15 & 15 & 16 & 5.2 & 6 & 6.2 \\
\hline 6-18 & White River at Mile 11.5, Ark. & 3.5 & - & 3.5 & 8 & - & 8.2 \\
\hline $6-19$ & Arkansas River at Pendleton, Ark. & 82 & -- & 85 & 5.9 & - & 5.7 \\
\hline $6-20$ & Mississippi River above Arkansas City, Ark. & 22 & 22 & 24 & 6 & 6 & 5.8 \\
\hline $6-22$ & Yazoo River below Steele Bayou, Miss. & 2.6 & 2.6 & 2.5 & 7 & 7.1 & 7.2 \\
\hline $6-23$ & Mississippi River below Vicksburg, Miss. & 20 & 20 & 20 & 5.7 & 5.8 & 5.6 \\
\hline $6-25$ & $\begin{array}{l}\text { Old River Outflow Channel near Knox } \\
\text { Landing, La. }\end{array}$ & 19 & 19 & 19 & 5.6 & 5.6 & 5.5 \\
\hline $6-26$ & Mississippi River near St. Francisville, La. & 19 & 19 & 19 & 5.6 & 5.7 & 5.7 \\
\hline $6-28$ & Mississippi River below Belle Chasse, La. & 21 & 19 & 22 & 5.3 & 5 & 5.4 \\
\hline
\end{tabular}


Table 30.- Concentration data for dissolved major elements in water samples collected from the Mississippi River and some of its tributaries during February-March 1990 cruise

[ A, Composite A; B, Composite B; -, not determined; All results are in milligrams per liter ]

\begin{tabular}{|c|c|c|c|c|c|c|c|c|c|c|}
\hline \multirow{2}{*}{$\begin{array}{l}\text { Date } \\
1990\end{array}$} & \multirow[b]{2}{*}{ Sampling site } & \multicolumn{3}{|c|}{ Calcium } & \multicolumn{3}{|c|}{ Magnesium } & \multicolumn{3}{|c|}{ Potassium } \\
\hline & & A & B & Grab & A & B & Grab & A & B & Grab \\
\hline $2-23$ & Cumberland River near Smithland, Ky. & 31 & 30 & 28 & $\overline{4.2}$ & $\overline{4.1}$ & 3.8 & 1.5 & $\overline{1.5}$ & 1.4 \\
\hline $2-24$ & Tennessee River near Calvert City, Ky. & 18 & 17 & 17 & 3 & 2.9 & 3 & 1.3 & 1.3 & 1.5 \\
\hline $2-25$ & Mississippi River near Cache, Ill. & 41 & - & 47 & 20 & - & 22 & 4.5 & - & 4.5 \\
\hline $2-28$ & Wabash River near New Haven, Ill. & 46 & 44 & 52 & 16 & 16 & 18 & 3.2 & 3.2 & 3.1 \\
\hline 3-01 & Ohio River at Uniontown, Ky. & 33 & 33 & 34 & 9 & 9 & 9.1 & 2.4 & 2.3 & 2.2 \\
\hline $3-03$ & Ohio River at Olmsted, Ill. & 29 & 32 & 35 & 7.6 & 8.4 & 9.9 & 2.1 & 2.2 & 2.5 \\
\hline $3-04$ & Mississippi River below Hickman, Ky. & 34 & 33 & 36 & 9.7 & 9.4 & 14 & 2.7 & 2.8 & 2.9 \\
\hline 3-05 & Mississippi River below Fulton, Tenn. & 29 & 34 & 36 & 9.3 & 11 & 12 & 2.6 & 2.5 & 2.7 \\
\hline 3-07 & Mississippi River at Helena, Ark. & 33 & 29 & 31 & 11 & 9.2 & 10 & 2.8 & 2.4 & 2.4 \\
\hline 3-07 & Arkansas River at Mile 1.0, Ark. & 27 & - & - & 6.5 & - & -- & 2.8 & -- & - \\
\hline $3-08$ & Mississippi River above Arkansas City, Ark. & 31 & 31 & 27 & 9.9 & 11 & 8.6 & 2.3 & 2.4 & 2.5 \\
\hline 3-08 & White River at Mile 1.0, Ark. & 16 & - & - & 7.1 & - & - & 1.9 & - & - \\
\hline 3-10 & Yazoo River at Mile 1.0, Miss. & 12 & - & - & 3.4 & -- & - & 2.2 & - & - \\
\hline 3-10 & Mississippi River below Vicksburg, Miss. & 25 & 24 & 29 & 7.9 & 7.7 & 9.3 & 2.3 & 2.5 & 2.7 \\
\hline 3-12 & Mississippi River near St. Francisville, La. & 27 & 27 & 27 & 8.4 & 8.4 & 8.3 & 2.4 & 2.5 & 2.6 \\
\hline 3-14 & Mississippi River below Belle Chasse, La. & 27 & 27 & 27 & 8.2 & 8.2 & 8.4 & 2.5 & 2.4 & 2.5 \\
\hline
\end{tabular}

Table 30.- Concentration data for dissolved major elements in water samples collected from the Mississippi River and some of its tributaries during February-March 1990 cruise -- Continued

\begin{tabular}{|c|c|c|c|c|c|c|c|}
\hline \multirow{2}{*}{$\begin{array}{l}\text { Date } \\
1990\end{array}$} & \multirow[b]{2}{*}{ Sampling site } & \multicolumn{3}{|c|}{ Sodium } & \multicolumn{3}{|c|}{ Silicon (as $\mathrm{SiO}_{2}$ ) } \\
\hline & & A & B & Grab & A & B & Grab \\
\hline $2-23$ & Cumberland River near Smithland, Ky. & 3 & 3 & 2.8 & 5.1 & 5 & 4.7 \\
\hline 2-24 & Tennessee River near Calvert City, Ky. & 3.5 & 3.5 & 3.5 & 5.4 & 5.3 & 5.4 \\
\hline $2-25$ & Mississippi River near Cache, Ill. & 23 & - & 27 & 6.5 & - & 7.5 \\
\hline $2-28$ & Wabash River near New Haven, Ill. & 8 & 7.8 & 8.6 & 7.2 & 7.1 & 8.1 \\
\hline 3-01 & Ohio River at Uniontown, Ky. & 9.3 & 9.2 & 9.3 & 6.7 & 6.7 & 6.7 \\
\hline $3-03$ & Ohio River at Olmsted, Ill. & 6.7 & 7.3 & 8.4 & 6.3 & 6.8 & 7.1 \\
\hline 3-04 & Mississippi River below Hickman, Ky. & 11 & 10 & 12 & 6.6 & 6.4 & 6.8 \\
\hline $3-05$ & Mississippi River below Fulton, Tenn. & 9 & 11 & 11 & 6.2 & 7.2 & 7.3 \\
\hline 3-07 & Mississippi River at Helena, Ark. & 10 & 9 & 9.7 & 7 & 6.1 & 6.5 \\
\hline $3-07$ & Arkansas River at Mile 1.0, Ark. & 33 & - & - & 4 & - & - \\
\hline $3-08$ & Mississippi River above Arkansas City, Ark. & 12 & 12 & 13 & 6.6 & 6.7 & 5.7 \\
\hline 3-08 & White River at Mile 1.0, Ark. & 2.1 & -- & - & 5.7 & - & - \\
\hline $3-10$ & Yazoo River at Mile 1.0, Miss. & 5.1 & - & - & 5.7 & - & - \\
\hline $3-10$ & Mississippi River below Vicksburg, Miss. & 10 & 9.9 & 12 & 5.6 & 5.5 & 7.7 \\
\hline $3-12$ & Mississippi River near St. Francisville, La. & 11 & 11 & 11 & 5.8 & 5.8 & 5.7 \\
\hline $3-14$ & Mississippi River below Belle Chasse, La. & 12 & 12 & 12 & 5.8 & 5.8 & 6 \\
\hline
\end{tabular}




\section{Table 31.- Concentration data for dissolved major elements in water samples collected from the Mississippi River and some of its tributaries during the May-June 1990 cruise}

[ A, Composite A; B, Composite B; --, not determined; All results are in milligrams per liter ]

\begin{tabular}{|c|c|c|c|c|c|c|c|c|c|c|}
\hline \multirow{2}{*}{$\begin{array}{l}\text { Date } \\
1990\end{array}$} & \multirow[b]{2}{*}{ Sampling site } & \multicolumn{3}{|c|}{ Calcium } & \multicolumn{3}{|c|}{ Magnesium } & \multicolumn{3}{|c|}{ Potassium } \\
\hline & & $\bar{A}$ & B & Grab & $\mathbf{A}$ & $\mathbf{B}$ & Grab & A & $\mathbf{B}$ & Grab \\
\hline 6-07 & Illinois River at Valley City, Ill. & - & 77 & 47 & $\overline{-}$ & 33 & 17 & 3.1 & 3.1 & 3.6 \\
\hline $6-10$ & Missouri River at St. Charles, Mo. ${ }^{1}$ & 36 & _- & 37 & 9.3 & -- & 10 & 3.9 & - & 4.6 \\
\hline $6-11$ & Mississippi River below Grafton, Ill. & 50 & 50 & 51 & 18 & 18 & 18 & 3.7 & 3.7 & 3.6 \\
\hline $6-13$ & Mississippi River at Thebes, $\mathrm{nl}$. & 39 & 40 & 41 & 12 & 13 & 13 & 3.6 & 3.9 & 3.8 \\
\hline $6-14$ & Ohio River at Olmsted, Ill. & 35 & 34 & 34 & 8.4 & 8.2 & 8.4 & 2.4 & 2.7 & 2.5 \\
\hline $6-18$ & Mississippi River below Memphis, Tenn. & 38 & 38 & 38 & 11 & 11 & 11 & 3.6 & 3.5 & 3.3 \\
\hline $6-19$ & White River at Mile 1.0, Ark. & - & - & 26 & -- & - & 9.8 & - & - & 1.7 \\
\hline $6-19$ & Arkansas River at Mile 1.0, Ark. & - & - & 29 & - & - & 6.1 & - & - & 2.5 \\
\hline $6-20$ & Mississippi River below Arkansas City, Ark. & 37 & 37 & 36 & 9.8 & 11 & 11 & 3.2 & 3.3 & 3.1 \\
\hline $6-22$ & Yazoo River below Steele Bayou, Miss. & 11 & 11 & 11 & 3.3 & 3.4 & 3.4 & 3.2 & 3.2 & 3.2 \\
\hline $6-23$ & Mississippi River below Vicksburg, Miss. & 35 & 36 & 35 & 9.9 & 10 & 9.9 & 3.4 & 3.3 & 3.3 \\
\hline $6-25$ & Mississippi River near St. Francisville, La. & 35 & 36 & 36 & 9.8 & 10 & 10 & 3.3 & 3.4 & 3.4 \\
\hline 6-27 & Mississippi River below Belle Chasse, La. & 35 & 36 & 36 & 9.9 & 11 & 11 & 3.3 & 3.5 & 3.4 \\
\hline
\end{tabular}

Table 31.- Concentration data for dissolved major elements in water samples collected from the Mississippi River and some of its tributaries during the May-June 1990 cruise - Continued

\begin{tabular}{|c|c|c|c|c|c|c|c|}
\hline \multirow{2}{*}{$\begin{array}{l}\text { Date } \\
1990\end{array}$} & \multirow[b]{2}{*}{ Sampling site } & \multicolumn{3}{|c|}{ Sodium } & \multicolumn{3}{|c|}{ Silicon (as $\mathrm{SiO}_{2}$ ) } \\
\hline & & A & B & Grab & $\bar{A}$ & B & Grab \\
\hline 6-07 & Illinois River at Valley City, Ill. & - & 26 & 9.3 & - & 6.4 & 6.8 \\
\hline $6-10$ & Missouri River at St. Charles, Mo. ${ }^{1}$ & 16 & - & 17 & 7.9 & - & 16 \\
\hline $6-11$ & Mississippi River below Grafton, Ill. & 11 & 11 & 11 & 6.7 & 6.6 & 6.4 \\
\hline $6-13$ & Mississippi River at Thebes, Ill. & 13 & 13 & 13 & 7.4 & 7.5 & 7.6 \\
\hline $6-14$ & Ohio River at Olmsted, Ill. & 7.3 & 7 & 7.1 & 6 & 5.5 & 5.6 \\
\hline $6-18$ & Mississippi River below Memphis, Tenn. & 11 & 11 & 11 & 6.9 & 6.7 & 6.6 \\
\hline $6-19$ & White River at Mile 1.0, Ark. & - & - & 2.2 & - & - & 7.1 \\
\hline $6-19$ & Arkansas River at Mile 1.0, Ark. & - & - & 15 & - & - & 6.2 \\
\hline $6-20$ & Mississippi River below Arkansas City, Ark. & 11 & 11 & 11 & 6.9 & 9.2 & 6.8 \\
\hline $6-22$ & Yazoo River below Steele Bayou, Miss. & 4.8 & 4.9 & 5 & 6.5 & 6.5 & 6.6 \\
\hline $6-23$ & Mississippi River below Vicksburg, Miss. & 10 & 11 & 10 & 6.7 & 6.7 & 6.7 \\
\hline $6-25$ & Mississippi River near St. Francisville, La. & 10 & 11 & 11 & 6.7 & 7.2 & 6.9 \\
\hline $6-27$ & Mississippi River below Belle Chasse, La. & 12 & 13 & 13 & 6.8 & 7 & 7 \\
\hline
\end{tabular}

${ }^{1}$ Single vertical composite near centroid of flow 
Table 32.- Concentration data for dissolved chloride, sulfate, and nitrate (as nitrogen) in water samples collected from the Mississippi River and some of its tributaries during the July-August 1987 cruise

[ A, Composite A; B, Composite B; --, not determined; All results are in milligrams per liter ]

\begin{tabular}{|c|c|c|c|c|c|c|c|c|c|c|}
\hline \multirow{2}{*}{$\begin{array}{l}\text { Date } \\
1987\end{array}$} & \multirow[b]{2}{*}{ Sampling site } & \multicolumn{3}{|c|}{ Chloride } & \multicolumn{3}{|c|}{ Sulfate } & \multicolumn{3}{|c|}{ Nitrate as nitrogen } \\
\hline & & $\bar{A}$ & B & Grab & $\mathbf{A}$ & B & Grab & A & B & Grab \\
\hline $7-17$ & Illinois River below Meredosia, Ill. & - & - & - & -- & -- & - & - & - & - \\
\hline $7-18$ & Mississippi River near Winfield, Mo. & 16 & - & 16 & 35 & 36 & -- & 1.7 & - & 2.1 \\
\hline $7-20$ & Missouri River at Hermann, Mo. & 17 & 18 & - & 81 & 76 & -- & 1.8 & 1.9 & - \\
\hline $7-21$ & Mississippi River at Hartford, Ill. & 20 & 20 & - & 40 & 40 & - & 2.0 & 1.9 & - \\
\hline $7-22$ & Mississippi River at St. Louis, Mo. & 20 & 20 & - & 66 & 67 & - & 1.5 & 1.8 & - \\
\hline $7-23$ & Mississippi River at Chester, Ill. & 19 & 19 & - & 65 & 62 & - & 1.9 & 1.6 & - \\
\hline $7-26$ & $\begin{array}{l}\text { Ohio River below Smithland Locks and Dam, } \\
\text { Ill.-Ky. }\end{array}$ & 20 & - & - & 71 & - & - & 1.8 & - & - \\
\hline $7-27$ & Ohio River at Olmsted, Ill. & 14 & 14 & - & 46 & 45 & - & 1.0 & 1.0 & - \\
\hline $7-28$ & Mississippi River below Hickman, Ky. & 18 & 18 & - & 69 & 69 & - & 1.6 & 1.6 & - \\
\hline $7-28$ & $\begin{array}{l}\text { Mississippi River above Hatchie River, } \\
\text { Tenn. }\end{array}$ & - & - & 16 & - & - & 55 & - & - & 1.4 \\
\hline $7-28$ & Hatchie River at Highway 61 bridge, Tenn. & - & - & 6.4 & -- & - & 4.2 & - & - & 0.7 \\
\hline $7-30$ & Mississippi River at Helena, Ark. & 16 & 15 & - & 53 & 50 & - & 1.4 & 1.4 & - \\
\hline $7-31$ & White River at Mile 11.5, Ark. & 6.2 & 6.2 & - & 7.5 & 7.3 & - & 0.3 & 0.3 & - \\
\hline $8-01$ & Arkansas River at Mile 55.9, Ark. & 132 & 133 & - & 90 & 92 & - & 0.6 & 0.4 & - \\
\hline $8-02$ & Mississippi River above Arkansas City, Ark. & 30 & 30 & - & 61 & 62 & - & 1.6 & 1.8 & - \\
\hline $8-04$ & Yazoo River at Highway 61, Miss. & - & - & 7.5 & - & - & 1.0 & - & -- & - \\
\hline 8-04 & Mississippi River below Vicksburg, Miss. & 28 & 27 & - & 59 & 57 & - & 1.5 & 1.4 & - \\
\hline $8-06$ & $\begin{array}{l}\text { Old River Outflow Channel near Knox } \\
\text { Landing, La. }\end{array}$ & 27 & 27 & - & 56 & 56 & - & 1.3 & 1.4 & - \\
\hline $8-07$ & Mississippi River near St. Francisville, La. & 26 & 27 & - & 55 & 56 & & 1.5 & 1.3 & -- \\
\hline $8-08$ & Mississippi River at Plaquemine, La. & - & - & 25 & -- & - & 51 & - & - & 1.2 \\
\hline $8-08$ & Mississippi River at Union, La. & - & - & 28 & -- & - & 51 & - & - & 1.2 \\
\hline $8-08$ & Mississippi River at Luling Bridge, La. & - & - & 25 & - & - & 51 & - & - & 1.3 \\
\hline 8-09 & Mississippi River below Belle Chasse, La. & & - & 42 & -- & - & 58 & 1.4 & - & - \\
\hline
\end{tabular}


Table 33.- Concentration data for dissolved chloride, sulfate, and nitrate (as nitrogen) in water samples collected from the Mississippi River and some of its tributaries during the NovemberDecember 1987 cruise

[ A, Composite A; B, Composite B; -, not determined; All results are in milligrams per liter ]

\begin{tabular}{|c|c|c|c|c|c|c|c|c|c|c|}
\hline \multirow{2}{*}{$\begin{array}{l}\text { Date } \\
1987\end{array}$} & \multirow[b]{2}{*}{ Sampling site } & \multicolumn{3}{|c|}{ Chloride } & \multicolumn{3}{|c|}{ Sulfate } & \multicolumn{3}{|c|}{ Nitrate as nitrogen } \\
\hline & & A & B & Grab & A & B & Grab & A & $\mathbf{B}$ & Grab \\
\hline $11-29$ & Illinois River below Meredosia, Ill. & 58 & - & - & 80 & - & - & 3.2 & - & - \\
\hline $11-30$ & Mississippi River near Winfield, Mo. & 18 & 18 & - & 29 & 29 & - & 1.6 & 1.5 & - \\
\hline $12-02$ & Missouri River at St. Charles, Mo. & 16 & 15 & - & 126 & 126 & -- & 1.2 & 1.1 & - \\
\hline $12-03$ & Mississippi River at St. Louis, Mo. & 19 & 18 & - & 76 & 71 & - & 1.5 & 1.4 & - \\
\hline $12-05$ & Mississippi River at Thebes, Ill. & 19 & 20 & - & 78 & 79 & - & 1.5 & 1.5 & - \\
\hline $12-06$ & Ohio River at Olmsted, Ill. & 25 & 26 & - & 70 & 71 & -. & 1.3 & 1.3 & - \\
\hline 12-07 & Mississippi River below Hickman, Ky. & 22 & 22 & - & 71 & 71 & - & 1.4 & 1.4 & - \\
\hline $12-08$ & Mississippi River at Fulton, Tenn. & 21 & 21 & - & 70 & 70 & - & 1.3 & 1.3 & - \\
\hline $12-11$ & Mississippi River at Helena, Ark. & 21 & 21 & - & 69 & 67 & - & 1.4 & 1.3 & - \\
\hline $12-12$ & White River at Mile 11.5, Ark. & 6.1 & 7.3 & - & 8.1 & 8.2 & - & 0.4 & 0.4 & - \\
\hline $12-13$ & Mississippi River above Arkansas City, Ark. & 26 & 27 & - & 61 & 61 & - & 1.3 & 1.2 & - \\
\hline $12-14$ & Yazoo River at Mile 10, Miss. & & & - & & & - & 0.5 & 0.5 & - \\
\hline $12-15$ & Mississippi River below Vicksburg, Miss. & 27 & 26 & -- & 62 & 59 & - & 1.2 & 1.2 & -- \\
\hline $12-17$ & $\begin{array}{l}\text { Old River Outflow Channel near Knox Landing, } \\
\text { La. }\end{array}$ & & & - & & & -- & 1.2 & 1.2 & - \\
\hline $12-18$ & Mississippi River near St. Francisville, La. & 27 & 27 & -- & 62 & 63 & - & 1.3 & 1.3 & - \\
\hline $12-20$ & Mississippi River below Belle Chasse, La. & 40 & 40 & -- & 61 & 61 & - & 1.3 & 1.3 & - \\
\hline
\end{tabular}

Table 34.- Concentration data for dissolved chloride, sulfate, and nitrate (as nitrogen) in water samples collected from the Mississippi River and some of its tributaries during the May-June 1988 cruise

[ A, Composite A; B, Composite B; --, not determined; <, less than the detection limit; All results are in milligrams per liter ]

\begin{tabular}{|c|c|c|c|c|c|c|c|c|c|c|}
\hline \multirow{2}{*}{$\begin{array}{l}\text { Date } \\
1988\end{array}$} & \multirow[b]{2}{*}{ Sampling site } & \multicolumn{3}{|c|}{ Chloride } & \multicolumn{3}{|c|}{ Sulfate } & \multicolumn{3}{|c|}{ Nitrate as nitrogen } \\
\hline & & A & B & Grab & A & B & Grab & A & B & Grab \\
\hline $5-16$ & Illinois River below Meredosia, Ill. & 58 & 56 & 58 & 85 & 85 & 87 & 3.4 & 3.3 & 3.2 \\
\hline $5-17$ & Mississippi River near Winfield, Mo. & 18 & 17 & 17 & 37 & 36 & 37 & 1.3 & 1.2 & 1.3 \\
\hline $5-19$ & Missouri River at Hermann, Mo. & 19 & 19 & - & 143 & 152 & - & 0.9 & 0.9 & - \\
\hline $5-20$ & Mississippi River at St. Louis, Mo. & 22 & 22 & - & 89 & 89 & - & 1.3 & 1.2 & - \\
\hline $5-22$ & Mississippi River at Thebes, Ill. & 22 & 22 & - & 90 & 91 & - & 1.3 & 1.4 & - \\
\hline $5-23$ & Ohio River at Olmsted, Ill. & 18 & 12 & -- & 59 & 40 & -- & 0.8 & 0.6 & -- \\
\hline $5-24$ & Mississippi River below Hickman, Ky. & 20 & 20 & 20 & 76 & 76 & 80 & 1.2 & 1.2 & 1.1 \\
\hline $5-26$ & Mississippi River at Fulton, Tenn. & 19 & 19 & 14 & 73 & 72 & 48 & 1.0 & 0.9 & 0.8 \\
\hline $5-28$ & Mississippi River at Helena, Ark. & 17 & 19 & 22 & 59 & 68 & 81 & 0.9 & 1.0 & 1.2 \\
\hline $5-29$ & White River at Mile 11.5, Ark. & 6.2 & 6.8 & 5.7 & 7.4 & 7.2 & 7.3 & $<0.009$ & $<0.009$ & $<0.009$ \\
\hline $5-29$ & Arkansas River at Mile 1.0, Ark. & _- & - & 90 & - & - & 58 & - & - & 0.4 \\
\hline $5-30$ & Mississippi River above Arkansas City, Ark. & 19 & 20 & 16 & 66 & 67 & 58 & 1.0 & 1.0 & 0.8 \\
\hline 6-01 & Yazoo River at Mile 10, Miss. & 7.1 & - & 7.1 & 9.2 & - & 9.4 & $<0.009$ & - & $<0.009$ \\
\hline 6-02 & Mississippi River below Vicksburg, Miss. & 20 & 19 & 19 & 65 & 64 & 65 & 0.9 & 0.9 & 0.8 \\
\hline $6-04$ & $\begin{array}{l}\text { Old River Outflow Channel near Knox Landing, } \\
\text { La. }\end{array}$ & 19 & 19 & 21 & 65 & 65 & 69 & 0.9 & 0.9 & 0.9 \\
\hline 6-05 & Mississippi River near St. Francisville, La. & 12 & 13 & 21 & 39 & 30 & 57 & 0.6 & 0.6 & 0.8 \\
\hline 6-07 & Mississippi River below Belle Chasse, La. & 32 & 31 & - & 64 & 62 & - & 1.0 & 1.0 & - \\
\hline
\end{tabular}


Table 35.- Concentration data for dissolved chloride and sulfate in water samples collected from the Mississippi River and some of its tributaries during the March-April 1989 cruise

[ A, Composite A; B, Composite B; --, not determined; All results are in milligrams per liter ]

\begin{tabular}{|c|c|c|c|c|c|c|c|}
\hline \multirow{2}{*}{$\begin{array}{l}\text { Date } \\
1989\end{array}$} & \multirow[b]{2}{*}{ Sampiing site } & \multicolumn{3}{|c|}{ Chioride } & \multicolumn{3}{|c|}{ Suifate } \\
\hline & & $\mathbf{A}$ & $\mathbf{B}$ & Grab & A & B & Grab \\
\hline $3-09$ & Illinois River at Hardin, Ill. & 109 & 104 & 105 & 109 & 101 & 109 \\
\hline $3-10$ & Mississippi River near Winfield, Mo. & 24.4 & - & 25 & 30.6 & - & 31.0 \\
\hline $3-12$ & Missouri River at Hermann, Mo. & 16.9 & 16.7 & 15.5 & 78.2 & 72.5 & 66.4 \\
\hline $3-13$ & Mississippi River at St. Louis, Mo. & 41.4 & 40.7 & -- & 58.4 & 61.7 & - \\
\hline $3-15$ & Mississippi River at Thebes, Ill. & 34 & 33.2 & 33.8 & 61 & 58.5 & 60.0 \\
\hline $3-16$ & Ohio River at Olmsted, Ill. & 9.5 & 10.0 & 8.8 & 36.1 & 37.8 & 35.2 \\
\hline $3-17$ & Mississippi River below Hickman, Ky. & 14.1 & 14.5 & - & 39.9 & 40.8 & - \\
\hline $3-19$ & Mississippi River below Fulton, Tenn. & 14.3 & 14.2 & 15.0 & 41.2 & 40.3 & 42.3 \\
\hline $3-21$ & Mississippi River at Helena, Ark. & 14.3 & 14.3 & 13.0 & 39.7 & 39.4 & 36.7 \\
\hline $3-22$ & White River at Mile 11.5, Ark. & 3.1 & 4.1 & 3.0 & 5.3 & 5.7 & 5.3 \\
\hline $3-23$ & Arkansas River at Pendleton, Ark. & 42.2 & 41.6 & 44.3 & 31.0 & 31.5 & 32.6 \\
\hline $3-24$ & Mississippi River above Arkansas City, Ark. & 15.7 & 16.3 & 16.6 & 37.2 & 38.2 & 36.6 \\
\hline $3-26$ & Yazoo River below Steele Bayou, Miss. & 2.1 & 2.1 & 3.8 & 5.1 & 5.2 & 5.8 \\
\hline $3-27$ & Mississippi River below Vicksburg, Miss. & 15.7 & 16.0 & 15.8 & 37.9 & 38.4 & 37.9 \\
\hline 3-29 & $\begin{array}{l}\text { Old River Outflow Channel near Knox } \\
\text { Landing, La. }\end{array}$ & 15.5 & 15.5 & 15.1 & 37.0 & 37.0 & 36.3 \\
\hline $3-30$ & Mississippi River near St. Francisville, La. & 15.6 & 14.6 & 14.9 & 37.2 & 35.6 & 36.3 \\
\hline 4-01 & Mississippi River below Belle Chasse, La. & 17.3 & 16.6 & 17.8 & 35.9 & 34.4 & 36.9 \\
\hline
\end{tabular}

Table 36.-- Concentration data for dissolved chloride and sulfate in water samples collected from the Mississippi River and some of its tributaries during the June 1989 cruise

[ A, Composite A; B, Composite B; -, not determined; All results are in milligrams per liter ]

\begin{tabular}{|c|c|c|c|c|c|c|c|}
\hline \multirow{2}{*}{$\begin{array}{l}\text { Date } \\
1989\end{array}$} & \multirow[b]{2}{*}{ Sampling site } & \multicolumn{3}{|c|}{ Chloride } & \multicolumn{3}{|c|}{ Sulfate } \\
\hline & & A & B & Grab & A & B & Grab \\
\hline $6-04$ & Illinois River at Hardin, Ill. & - & - & - & - & - & - \\
\hline $6-05$ & Mississippi River near Winfield, Mo. & - & - & - & - & - & - \\
\hline $6-07$ & Missouri River at Hermann, Mo. & - & - & - & - & - & - \\
\hline $6-08$ & Mississippi River at St. Louis, Mo. & 25.4 & 24.8 & - & 82.3 & 82.5 & 20.4 \\
\hline $6-10$ & Mississippi River at Thebes, Ill. & 25.3 & 25.9 & 26.2 & 79.2 & 82.8 & 80.3 \\
\hline $6-11$ & Ohio River at Olmsted, Ill. & 12.3 & 12.6 & 15.8 & 46.4 & 46.6 & 52.8 \\
\hline $6-12$ & Mississippi River below Hickman, Ky. & 15.8 & 17.2 & 15.5 & 54.5 & 54.5 & 52.8 \\
\hline $6-14$ & Mississippi River at Fulton, Tenn. & 15.9 & 15.9 & 16.6 & 53.2 & 53.2 & 52.2 \\
\hline $6-17$ & Mississippi River at Helena, Ark. & 14.7 & 14.5 & 14.0 & 46.9 & 46.4 & 48.7 \\
\hline $6-18$ & White River at Mile 11.5, Ark. & 4.0 & 4.0 & - & 5.1 & 5.1 & - \\
\hline $6-19$ & Arkansas River at Pendleton, Ark. & 103 & - & - & 45.5 & - & - \\
\hline $6-20$ & Mississippi River above Arkansas City, Ark. & 29.3 & 28.6 & - & 39.2 & 41.4 & - \\
\hline $6-22$ & Yazoo River below Steele Bayou, Miss. & 4.0 & - & 2.7 & 4.4 & - & 4.0 \\
\hline $6-23$ & Mississippi River below Vicksburg, Miss. & 23.1 & 26.2 & 25.4 & 33.9 & 38.4 & 38.7 \\
\hline $6-25$ & $\begin{array}{l}\text { Old River Outflow Channel near Knox } \\
\text { Landing, La. }\end{array}$ & 25.4 & 25.7 & 25.2 & 38.7 & 37.8 & 36.8 \\
\hline $6-26$ & Mississippi River near St. Francisville, La. & 23.4 & 23.4 & 24.2 & 35.7 & 35.9 & 36.6 \\
\hline $6-28$ & Mississippi River below Belle Chasse, La. & 29.7 & 29.2 & 30.0 & 36.1 & 38.8 & 38.7 \\
\hline
\end{tabular}


Table 37.- Concentration data for dissolved chloride, sulfate, and nitrate (as nitrogen) in water samples collected from the Mississippi River and some of its tributaries during the FebruaryMarch 1990 cruise

[ A, Composite A; B, Composite B; --, not determined; <, less than the detection limit; All results are in milligrams per liter ]

\begin{tabular}{|c|c|c|c|c|c|c|c|c|c|c|}
\hline \multirow{2}{*}{$\begin{array}{l}\text { Date } \\
1990 \\
\end{array}$} & \multirow[b]{2}{*}{ Sampling site } & \multicolumn{3}{|c|}{ Chioride } & \multicolumn{3}{|c|}{ Sulfate } & \multicolumn{3}{|c|}{ Nitrate as nitrogen } \\
\hline & & A & $\mathbf{B}$ & Grab & A & B & Grab & A & $\mathbf{B}$ & Grab \\
\hline $2-23$ & Cumberland River near Smithland, Ky. & 3.3 & 4.0 & - & 17.2 & 17.9 & -- & 0.8 & 1.0 & - \\
\hline $2-24$ & Tennessee River near Calvert City, Ky. & 4.2 & 4.3 & 4.3 & 9.8 & 9.6 & 9.6 & 0.6 & 0.6 & 0.6 \\
\hline $2-25$ & Mississippi River near Cache, Ill. & 31.7 & - & 32.5 & 63.2 & - & 62.9 & 2.7 & -- & 2.3 \\
\hline $2-28$ & Wabash River near New Haven, Ill. & 18.3 & 17.6 & 16.5 & 43.1 & 41.5 & 40.0 & 4.4 & 4.6 & 4.4 \\
\hline 3-01 & Ohio River at Uniontown, Ky. & 13.5 & 12.5 & 12.5 & 44.8 & 49.6 & 49.9 & 2.2 & 1.8 & 1.9 \\
\hline $3-03$ & Ohio River at Olmsted, Ill. & 10.3 & 10.3 & 12.6 & 36.4 & 35.4 & 45.2 & 1.9 & 1.9 & 2.3 \\
\hline 3-04 & Mississippi River below Hickman, Ky. & - & - & - & -- & - & - & - & - & - \\
\hline 3-05 & Mississippi River below Fulton, Tenn. & - & 12.8 & 13.1 & - & 37.4 & 37.7 & 1.8 & 1.9 & 1.9 \\
\hline 3-07 & Mississippi River at Helena, Ark. & 13.3 & - & 13.4 & 37.6 & - & 38.0 & 1.9 & -- & 1.8 \\
\hline 3-07 & Arkansas River at Mile 1.0, Ark. & - & - & 54.6 & - & - & 31.0 & - & - & 0.1 \\
\hline 3-08 & Mississippi River above Arkansas City, Ark. & -- & 18.9 & 18.8 & - & 32.8 & 32.2 & -- & 1.6 & 1.2 \\
\hline $3-08$ & White River at Mile 1.0, Ark. & - & - & 2.7 & - & - & 4.8 & - & - & 0.1 \\
\hline 3-10 & Yazoo River at Mile 1.0, Miss. & - & - & 4.0 & - & - & 7.3 & - & - & $<0.01$ \\
\hline $3-10$ & Mississippi River below Vicksburg, Miss. & 16.0 & - & 16.1 & 31.9 & - & 32.2 & 1.3 & -- & 1.3 \\
\hline $3-12$ & Mississippi River near St. Francisville, La. & 15.2 & 15.3 & 13.2 & 31.1 & 31.1 & 26.7 & 1.0 & 0.7 & 1.1 \\
\hline 3-14 & Mississippi River below Belle Chasse, La. & 17.8 & 17.6 & 18.5 & 31.5 & 31.1 & 30.8 & 1.3 & 1.1 & 1.3 \\
\hline
\end{tabular}

Table 38. - Concentration data for dissolved chloride, sulfate, and nitrate (as nitrogen) in water samples collected from the Mississippi River and some of its tributaries during the May-June 1990 cruise

[ A, Composite A; B, Composite B; --, not determined; All results are in milligrams per liter ]

\begin{tabular}{|c|c|c|c|c|c|c|c|c|c|c|}
\hline \multirow{2}{*}{$\begin{array}{l}\text { Date } \\
1990\end{array}$} & \multirow[b]{2}{*}{ Sampling site } & \multicolumn{3}{|c|}{ Chloride } & \multicolumn{3}{|c|}{ Sulfate } & \multicolumn{3}{|c|}{ Nitrate as nitrogen } \\
\hline & & A & B & Grab & A & B & Grab & A & B & Grab \\
\hline $6-07$ & Illinois River at Valley City, Ill. & 46.0 & 43.7 & 46.1 & 75.8 & 73.8 & 75.1 & 8.4 & 8.2 & 8.3 \\
\hline $6-10$ & Missouri River at St. Charles, Mo. ${ }^{1}$ & 7.4 & - & 8.6 & 47.2 & - & 44.8 & 1.4 & - & 1.4 \\
\hline $6-11$ & Mississippi River below Grafton, Ill. & 20.3 & 19.6 & 20.5 & 36.1 & 34.7 & 35.2 & 5.7 & 5.7 & 5.9 \\
\hline $6-13$ & Mississippi River at Thebes, Ill. & 11.7 & 12.0 & 13.6 & 38.8 & 38.8 & 39.1 & 2.8 & 2.9 & 3.2 \\
\hline 6-14 & Ohio River at Olmsted, Ill. & 8.4 & 8.4 & 8.7 & 28.4 & 32.1 & 33.3 & 1.5 & 1.5 & 1.7 \\
\hline $6-18$ & Mississippi River below Memphis, Tenn. & 11.3 & 11.1 & 11.5 & 38.2 & 36.6 & 37.3 & 2.2 & 2.2 & 2.1 \\
\hline $6-19$ & White River at Mile 1.0, Ark. & - & - & 2.4 & - & -- & 4.1 & - & -- & 0.29 \\
\hline $6-19$ & Arkansas River at Mile 1.0, Ark. & - & - & 17.4 & -- & -- & 21.6 & -- & - & 0.63 \\
\hline $6-20$ & Mississippi River below Arkansas City, Ark. & 11.2 & 11.3 & 11.6 & 33.5 & 33.5 & 32.2 & 1.9 & 1.9 & 1.8 \\
\hline $6-22$ & Yazoo River below Steele Bayou, Miss. & 3.3 & 3.3 & 3.3 & 6.4 & 6.4 & 6.4 & 0.5 & 0.6 & 0.5 \\
\hline $6-23$ & Mississippi River below Vicksburg, Miss. & 11.4 & 11.1 & 11.1 & 31.7 & 32.0 & 31.0 & 1.8 & 1.7 & 1.7 \\
\hline $6-25$ & Mississippi River near St. Francisville, La. & 10.2 & 11.3 & 11.1 & 31.5 & 32.1 & 32.1 & 1.7 & 1.7 & 1.8 \\
\hline 6-27 & Mississippi River below Belle Chasse, La. & 14.9 & 10.2 & 14.7 & 32.9 & 32.3 & 32.7 & 1.7 & 1.7 & 2.0 \\
\hline
\end{tabular}

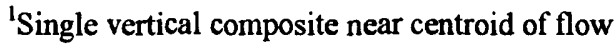


Table 39.- Concentration data for dissolved bicarbonate and carbonate in water samples collected from the Mississippi River and some of its tributaries during the July-August 1987 cruise

[ A, Composite A; B, Composite B; -, not determined; <, less than the detection limit; All results are in milligrams per liter ]

\begin{tabular}{|c|c|c|c|c|c|c|c|}
\hline \multirow{2}{*}{$\begin{array}{l}\text { Date } \\
1987\end{array}$} & \multirow[b]{2}{*}{ Sampling site } & \multicolumn{3}{|c|}{ Bicarbonate } & \multicolumn{3}{|c|}{ Carbonate } \\
\hline & & $\bar{A}$ & B & Grab & A & B & Grab \\
\hline $7-17$ & Illinois River below Meredosia, Ill. & - & - & - & - & - & - \\
\hline $7-18$ & Mississippi River near Winfield, Mo. & 181 & - & 178 & 0.90 & - & $<0.50$ \\
\hline $7-20$ & Missouri River at Hermann, Mo. & 138 & 143 & -- & $<0.05$ & $<0.05$ & - \\
\hline $7-21$ & Mississippi River at Hartford, Ill. & 175 & 175 & - & 0.87 & 0.87 & - \\
\hline $7-22$ & Mississippi River at St. Louis, Mo. & 155 & 154 & - & 0.77 & 0.76 & - \\
\hline $7-23$ & Mississippi River at Chester, Ill. & 154 & 155 & -- & 0.76 & 0.77 & _- \\
\hline $7-26$ & $\begin{array}{l}\text { Ohio River below Smithland Locks and Dam, } \\
\text { Ill.-Ky. }\end{array}$ & 124 & - & - & 0.61 & - & - \\
\hline $7-27$ & Ohio River at Olmsted, Ill. & 95 & 95 & -- & $<0.05$ & $<0.05$ & - \\
\hline $7-28$ & Mississippi River below Hickman, Ky. & 147 & 147 & - & 0.73 & 0.73 & - \\
\hline $7-28$ & $\begin{array}{l}\text { Mississippi River above Hatchie River, } \\
\text { Tenn. }\end{array}$ & - & - & 133 & - & - & 0.66 \\
\hline $7-28$ & Hatchie River at Highway 61 bridge, Tenn. & -- & -- & 23 & -- & - & $<0.50$ \\
\hline $7-30$ & Mississippi River at Helena, Ark. & 136 & 136 & -- & 0.68 & 0.68 & - \\
\hline $7-31$ & White River at Mile 11.5, Ark. & 164 & 166 & & 0.82 & 0.82 & - \\
\hline 8-01 & Arkansas River at Mile 55.9, Ark. & 145 & 145 & - & 0.72 & 0.72 & - \\
\hline $8-02$ & Mississippi River above Arkansas City, Ark. & 147 & 146 & - & 0.73 & 0.73 & - \\
\hline $8-04$ & Yazoo River at Highway 61, Miss. & - & - & 131 & - & - & 0.65 \\
\hline 8-04 & Mississippi River below Vicksburg, Miss. & 149 & 148 & - & 0.74 & 0.74 & - \\
\hline 8-06 & $\begin{array}{l}\text { Old River Outflow Channel near Knox } \\
\text { Landing, La. }\end{array}$ & 150 & 151 & - & 0.74 & 0.75 & - \\
\hline $8-07$ & Mississippi River near St. Francisville, La. & 151 & 151 & - & 0.75 & 0.75 & - \\
\hline $8-08$ & Mississippi River at Plaquemine, La. & - & -- & 148 & - & - & 0.74 \\
\hline $8-08$ & Mississippi River at Union, La. & - & - & 145 & - & - & 0.72 \\
\hline 8-08 & Mississippi River at Luling Bridge, La. & - & - & 142 & -- & - & 0.70 \\
\hline 8-09 & Mississippi River below Belle Chasse, La. & 140 & - & - & 0.70 & - & -- \\
\hline
\end{tabular}


Table 40.- Concentration data for dissolved bicarbonate and carbonate in water samples collected from the Mississippi River and some of its tributaries during November-December 1987 cruise

[ A, Composite A; B, Composite B; -, not determined; <, less than the detection limit; All results are in milligrams per liter ]

\begin{tabular}{|c|c|c|c|c|c|c|}
\hline \multirow[b]{2}{*}{ Sampling site } & \multicolumn{3}{|c|}{ Bicarbonate } & \multicolumn{3}{|c|}{ Carbonate } \\
\hline & A & B & Grab & A & B & Grab \\
\hline 11-29 Illinois River below Meredosia, Ill. & 250 & - & - & 1.2 & - & - \\
\hline 11-30 Mississippi River near Winfield, Mo. & 196 & 196 & -- & 2.0 & 2.0 & -- \\
\hline 12-02 Missouri River at St. Charles, Mo. & 188 & 190 & -- & 1.9 & 0.94 & - \\
\hline 12-03 Mississippi River at St. Louis, Mo. & 194 & 194 & -- & 0.96 & 0.96 & - \\
\hline 12-05 Mississippi River at Thebes, IIl. & 193 & 193 & -- & 0.96 & 0.96 & - \\
\hline 12-06 Ohio River at Olmsted, Ill. & 97 & 97 & -- & $<0.50$ & $<0.50$ & - \\
\hline 12-07 Mississippi River below Hickman, Ky. & 147 & 147 & - & 0.73 & 0.73 & -- \\
\hline 12-08 Mississippi River at Fulton, Tenn. & 146 & 146 & -- & - & - & - \\
\hline 12-11 Mississippi River at Helena, Ark. & 145 & 142 & - & $<0.50$ & $<0.50$ & -- \\
\hline 12-12 White River at Mile 11.5, Ark. & 108 & 108 & -- & $<0.50$ & $<0.50$ & -- \\
\hline 12-13 Mississippi River above Arkansas City, Ark. & 136 & 139 & -- & $<0.50$ & $<0.50$ & -- \\
\hline 12-14 Yazoo River at Mile 10, Miss. & 35 & 35 & - & $<0.50$ & $<0.50$ & - \\
\hline 12-15 Mississippi River below Vicksburg, Miss. & 137 & 137 & -- & $<0.50$ & $<0.50$ & - \\
\hline $\begin{array}{l}\text { 12-17 Old River Outflow Channel near Knox } \\
\text { Landing, La. }\end{array}$ & 134 & 135 & - & $<0.50$ & $<0.50$ & - \\
\hline 12-18 Mississippi River near St. Francisville, La. & 134 & 134 & -- & $<0.50$ & $<0.50$ & - \\
\hline 12-20 Mississippi River below Belle Chasse, La. & 132 & 134 & - & $<0.50$ & $<0.50$ & - \\
\hline
\end{tabular}

Table 41.- Concentration data for dissolved bicarbonate and carbonate in water samples collected from the Mississippi River and some of its tributaries during May-June 1988 cruise

[ A, Composite A; B, Composite B; --, not determined; <, less than the detection limit; All results are in milligrams per liter ]

\begin{tabular}{|c|c|c|c|c|c|c|c|}
\hline \multirow{2}{*}{$\begin{array}{l}\text { Date } \\
1988\end{array}$} & \multirow[b]{2}{*}{ Sampling site } & \multicolumn{3}{|c|}{ Bicarbonate } & \multicolumn{3}{|c|}{ Carbonate } \\
\hline & & $\overline{\mathbf{A}}$ & B & Grab & A & B & Grab \\
\hline $5-16$ & Illinois River below Meredosia, Ill. & 237 & 239 & 239 & 2.4 & 1.2 & 1.2 \\
\hline $5-17$ & Mississippi River near Winfield, Mo. & 166 & 166 & 166 & 0.82 & 0.82 & 0.82 \\
\hline $5-19$ & Missouri River at Hermann, Mo. & 178 & 165 & -- & 0.89 & 0.82 & - \\
\hline $5-20$ & Mississippi River at St. Louis, Mo. & 190 & 172 & -- & 0.94 & 0.86 & - \\
\hline $5-22$ & Mississippi River at Thebes, Ill. & 159 & 190 & - & 0.79 & 0.95 & - \\
\hline $5-23$ & Ohio River at Olmsted, Ill. & 97 & 69 & -- & $<0.50$ & $<0.50$ & -- \\
\hline $5-24$ & Mississippi River below Hickman, Ky. & 147 & 148 & 171 & 0.73 & 0.73 & 0.85 \\
\hline $5-26$ & Mississippi River at Fulton, Tenn. & 149 & 121 & 149 & 0.74 & 0.60 & 0.74 \\
\hline $5-28$ & Mississippi River at Helena, Ark. & 138 & 127 & 151 & 0.68 & 0.63 & 0.75 \\
\hline $5-29$ & White River at Mile 11.5, Ark. & 159 & 159 & 160 & 0.79 & 0.79 & 0.79 \\
\hline $5-29$ & Arkansas River at Mile 1.0, Ark. & -- & - & 155 & -- & - & 0.77 \\
\hline $5-30$ & Mississippi River above Arkansas City, Ark. & 153 & 148 & 108 & 0.76 & $<0.50$ & 0.54 \\
\hline $6-01$ & Yazoo River at Mile 10, Miss. & 123 & -- & 123 & $<0.50$ & -- & $<0.50$ \\
\hline $6-02$ & Mississippi River below Vicksburg, Miss. & 117 & 119 & 105 & 0.58 & 0.59 & 0.52 \\
\hline $6-04$ & $\begin{array}{l}\text { Old River Outflow Channel near Knox } \\
\text { Landing, La. }\end{array}$ & 157 & 158 & 156 & 0.78 & 0.78 & 0.78 \\
\hline $6-05$ & Mississippi River near St. Francisville, La. & 94 & 107 & 108 & 0.47 & $<0.50$ & 0.53 \\
\hline $6-07$ & Mississippi River below Belle Chasse, La. & 164 & 117 & - & $<0.50$ & 0.58 & - \\
\hline
\end{tabular}


Table 42.- Concentration data for dissolved bicarbonate and carbonate in water samples collected from the Mississippi River and some of its tributaries during March-April 1989 cruise

[ A, Composite A; B, Composite B; -, not determined; <, less than the detection limit; All results are in milligrams per liter ]

\begin{tabular}{|c|c|c|c|c|c|c|c|}
\hline \multirow{2}{*}{$\begin{array}{l}\text { Date } \\
1989\end{array}$} & \multirow[b]{2}{*}{ Sampiing site } & \multicolumn{3}{|c|}{ Bicarbonate } & \multicolumn{3}{|c|}{ Carbonate } \\
\hline & & $\bar{A}$ & $\mathbf{B}$ & Grab & $\mathbf{A}$ & $\mathbf{B}$ & Grab \\
\hline 3-09 & Illinois River at Hardin, Ill. & - & - & - & - & - & - \\
\hline $3-10$ & Mississippi River near Winfield, Mo. & - & - & - & -- & - & - \\
\hline $3-12$ & Missouri River at Hermann, Mo. & 133 & 139 & - & 0.66 & 0.69 & - \\
\hline $3-13$ & Mississippi River at St. Louis, Mo. & 137 & 146 & - & $<0.5$ & $<0.5$ & - \\
\hline $3-15$ & Mississippi River at Thebes, Ill. & 131 & 142 & 161 & $<0.5$ & $<0.5$ & $<0.5$ \\
\hline $3-16$ & Ohio River at Olmsted, Ill. & 63.4 & 64.7 & 66.0 & $<0.5$ & $<0.5$ & $<0.5$ \\
\hline $3-17$ & Mississippi River below Hickman, Ky. & 83.7 & 84.9 & 81.6 & $<0.5$ & $<0.5$ & $<0.5$ \\
\hline 3-19 & Mississippi River below Fulton, Tenn. & 86.3 & 86.2 & 86.0 & $<0.5$ & $<0.5$ & $<0.5$ \\
\hline $3-21$ & Mississippi River at Helena, Ark. & 88.7 & 87.4 & 83.8 & $<0.5$ & $<0.5$ & $<0.5$ \\
\hline $3-22$ & White River at Mile 11.5, Ark. & 80.0 & 81.7 & 80.7 & $<0.5$ & $<0.5$ & $<0.5$ \\
\hline $3-23$ & Arkansas River at Pendleton, Ark. & 70.2 & 74.3 & 71.9 & $<0.5$ & $<0.5$ & $<0.5$ \\
\hline $3-24$ & Mississippi River above Arkansas City, Ark. & 96.5 & 98.1 & 96.8 & $<0.5$ & $<0.5$ & $<0.5$ \\
\hline $3-26$ & Yazoo River below Steele Bayou, Miss. & 28.1 & 27.1 & 32.2 & $<0.5$ & $<0.5$ & $<0.5$ \\
\hline $3-27$ & Mississippi River below Vicksburg, Miss. & 97.0 & 98.9 & 99.6 & $<0.5$ & $<0.5$ & $<0.5$ \\
\hline $3-29$ & $\begin{array}{l}\text { Old River Outflow Channel near Knox } \\
\text { Landing, La. }\end{array}$ & 96.5 & 96.4 & 96.2 & $<0.5$ & $<0.5$ & $<0.5$ \\
\hline $3-30$ & Mississippi River near St. Francisville, La. & 95.5 & 95.3 & 94.9 & $<0.5$ & $<0.5$ & $<0.5$ \\
\hline 4-01 & Mississippi River below Belle Chasse, La. & 93.4 & 94.1 & 95.5 & $<0.5$ & $<0.5$ & $<0.5$ \\
\hline
\end{tabular}

Table 43.- Concentration data for dissolved bicarbonate and carbonate in water samples collected from the Mississippi River and some of its tributaries during June 1989 cruise

[ A, Composite A; B, Composite B; -, not determined; <, less than the detection limit; All results are in milligrams per liter ]

\begin{tabular}{|c|c|c|c|c|c|c|c|}
\hline \multirow{2}{*}{$\begin{array}{l}\text { Date } \\
1989\end{array}$} & \multirow[b]{2}{*}{ Sampling site } & \multicolumn{3}{|c|}{ Bicarbonate } & \multicolumn{3}{|c|}{ Carbonate } \\
\hline & & $\bar{A}$ & $\mathbf{B}$ & Grab & $\mathbf{A}$ & B & Grab \\
\hline$\overline{6-04}$ & Illinois River at Hardin, Ill. & - & - & - & - & - & - \\
\hline $6-05$ & Mississippi River near Winfield, Mo. & - & - & - & - & - & - \\
\hline $6-07$ & Missouri River at Hermann, Mo. & - & 103 & 133 & - & $<0.5$ & $<0.5$ \\
\hline $6-08$ & Mississippi River at St. Louis, Mo. & 145 & 150 & 66.5 & 0.72 & $<0.5$ & 0.67 \\
\hline $6-10$ & Mississippi River at Thebes, Ill. & - & 164 & - & - & $<0.5$ & -- \\
\hline $6-11$ & Ohio River at Olmsted, Ill. & 88.9 & 75.9 & 56.7 & $<0.5$ & $<0.5$ & $<0.5$ \\
\hline $6-12$ & Mississippi River below Hickman, Ky. & 104 & 115 & 105 & $<0.5$ & $<0.5$ & $<0.5$ \\
\hline $6-14$ & Mississippi River at Fulton, Tenn. & 124 & 124 & 126 & $<0.5$ & $<0.5$ & 0.63 \\
\hline $6-17$ & Mississippi River at Helena, Ark. & 117 & 119 & 118 & $<0.5$ & 0.59 & $<0.5$ \\
\hline $6-18$ & White River at Mile 11.5, Ark. & - & - & 133 & - & - & 0.66 \\
\hline $6-19$ & Arkansas River at Pendleton, Ark. & 87.9 & - & - & $<0.5$ & - & - \\
\hline $6-20$ & Mississippi River above Arkansas City, Ark. & 107 & 107 & 107 & $<0.5$ & $<0.5$ & $<0.5$ \\
\hline $6-22$ & Yazoo River below Steele Bayou, Miss. & 20.9 & 20.7 & 21.8 & $<0.5$ & $<0.5$ & $<0.5$ \\
\hline $6-23$ & Mississippi River below Vicksburg, Miss. & 100 & 103 & - & $<0.5$ & 0.51 & - \\
\hline $6-25$ & $\begin{array}{l}\text { Old River Outflow Channel near Knox } \\
\text { Landing, La. }\end{array}$ & 101 & 102 & 101 & $<0.5$ & $<0.5$ & $<0.5$ \\
\hline $6-26$ & Mississippi River near St. Francisville, La. & 100 & 99.1 & 99.4 & 0.50 & $<0.5$ & $<0.5$ \\
\hline $6-28$ & Mississippi River below Belle Chasse, La. & 103 & - & 102 & 0.51 & - & $<0.5$ \\
\hline
\end{tabular}


Table 44. - Concentration data for dissolved bicarbonate and carbonate in water samples collected from the Mississippi River and some of its tributaries during the February-March 1990 cruise

[ A, Composite A; B, Composite B; -, not determined; <, less than the detection limit; All results are in milligrams per liter ]

\begin{tabular}{|c|c|c|c|c|c|c|c|}
\hline \multirow{2}{*}{$\begin{array}{l}\text { Date } \\
1990\end{array}$} & \multirow[b]{2}{*}{ Sampling site } & \multicolumn{3}{|c|}{ Bicarbonate } & \multicolumn{3}{|c|}{ Carbonate } \\
\hline & & $\mathbf{A}$ & B & Grab & A & B & Grab \\
\hline $2-23$ & Cumberland River near Smithland, Ky. & 96.5 & 93.7 & 96.8 & $<0.5$ & $<0.5$ & $<0.5$ \\
\hline $2-24$ & Tennessee River near Calvert City, Ky. & 55.5 & 55.4 & 55.8 & $<0.5$ & $<0.5$ & $<0.5$ \\
\hline $2-25$ & Mississippi River near Cache, Ill. & 174 & - & 177 & 0.87 & - & 0.88 \\
\hline $2-28$ & Wabash River near New Haven, Ill. & 147 & 148 & 150 & $<0.5$ & 0.74 & 0.75 \\
\hline 3-01 & Ohio River at Uniontown, Ky. & - & 77.2 & 76.9 & - & $<0.5$ & $<0.5$ \\
\hline 3-03 & Ohio River at Olmsted, Ill. & 83.3 & 85.8 & 94.8 & $<0.5$ & $<0.5$ & $<0.5$ \\
\hline 3-04 & Mississippi River below Hickman, Ky. & - & - & - & - & - & - \\
\hline 3-05 & Mississippi River below Fulton, Tenn. & 24.5 & 94.1 & 98.6 & $<0.5$ & $<0.5$ & $<0.5$ \\
\hline 3-07 & Mississippi River at Helena, Ark. & 99.0 & - & 99.2 & $<0.5$ & - & $<0.5$ \\
\hline 3-07 & Arkansas River at Mile 1.0, Ark. & - & - & 85.0 & $<0.5$ & - & - \\
\hline 3-08 & Mississippi River above Arkansas City, Ark. & - & 91.6 & 92.9 & - & $<0.5$ & $<0.5$ \\
\hline $3-08$ & White River at Mile 1.0, Ark. & - & - & 55.1 & 0.55 & - & - \\
\hline 3-10 & Yazoo & - & - & 32.2 & $<0.5$ & - & - \\
\hline 3-10 & Mississippi River below Vicksburg, Miss. & 91.0 & - & 93.5 & $<0.5$ & - & $<0.5$ \\
\hline $3-12$ & Mississippi River near St. Francisville, La. & 90.3 & 91.0 & 67.7 & $<0.5$ & $<0.5$ & $<0.5$ \\
\hline $3-14$ & Mississippi River below Belle Chasse, La. & 88.9 & 90.2 & 87.0 & $<0.5$ & $<0.5$ & $<0.5$ \\
\hline
\end{tabular}

Table 45.- Concentration data for dissolved bicarbonate and carbonate in water samples collected from the Mississippi River and some of its tributaries during the May-June 1990 cruise

[ A, Composite A; B, Composite B; -, not determined; <, less than the detection limit; All results are in milligrams per liter ]

\begin{tabular}{|c|c|c|c|c|c|c|c|}
\hline \multirow{2}{*}{$\begin{array}{l}\text { Date } \\
1990\end{array}$} & \multirow[b]{2}{*}{ Sampling site } & \multicolumn{3}{|c|}{ Bicarbonate } & \multicolumn{3}{|c|}{ Carbonate } \\
\hline & & $\mathbf{A}$ & $\mathbf{B}$ & Grab & $\mathbf{A}$ & $\mathbf{B}$ & Grab \\
\hline $6-07$ & Illinois River at Valley City, Ill. & 248 & 249 & 250 & 1.2 & 1.2 & 1.2 \\
\hline $6-10$ & Missouri River at St. Charles, Mo. ${ }^{1}$ & 120 & - & 115 & 0.59 & - & 0.57 \\
\hline $6-11$ & Mississippi River below Grafton, Ill. & 164 & 161 & 160 & 0.82 & 0.80 & $<0.5$ \\
\hline $6-13$ & Mississippi River at Thebes, Ill. & 129 & 130 & 130 & 0.64 & 0.65 & 0.64 \\
\hline $6-14$ & Ohio River at Olmsted, Ill. & 98.3 & 97.7 & 101 & $<0.5$ & $<0.5$ & 0.50 \\
\hline $6-18$ & Mississippi River below Memphis, Tenn. & 120 & 120 & 120 & 0.60 & 0.59 & 0.60 \\
\hline $6-19$ & White River at Mile 1.0, Ark. & - & - & 121 & - & - & 0.60 \\
\hline $6-19$ & Arkansas River at Mile 1.0, Ark. & - & - & 92.4 & - & - & $<0.5$ \\
\hline $6-20$ & Mississippi River below Arkansas City, Ark. & 117 & 117 & 117 & 0.58 & 0.58 & 0.58 \\
\hline $6-22$ & Yazoo River below Steele Bayou, Miss. & 45.2 & 46.7 & 45.9 & $<0.5$ & $<0.5$ & $<0.5$ \\
\hline $6-23$ & Mississippi River below Vicksburg, Miss. & 117 & 116 & 115 & 0.58 & 0.58 & 0.57 \\
\hline $6-25$ & Mississippi River near St. Francisville, La. & 115 & 116 & 116 & 0.57 & 0.58 & 0.58 \\
\hline $6-27$ & Mississippi River below Belle Chasse, La. & 115 & 115 & 115 & 0.57 & 0.57 & 0.57 \\
\hline
\end{tabular}

${ }^{1}$ Single vertical composite near centroid of flow 
Table 46. - Discharge and corresponding transport data for dissolved trace elements in water samples collected from the Mississippi River and some of its tributaries during the July-August 1987 cruise

[ *, concentration less than the detection limit; All results are in kilograms per day except discharge, which is in cubic meters per second ]

\begin{tabular}{|c|c|c|c|c|c|c|c|c|}
\hline $\begin{array}{l}\text { Date } \\
1987\end{array}$ & Sampling site & Discharge & Aluminum & Arsenic & Barium & Beryllium & Boron & Cadmium \\
\hline $7 \cdot 17$ & Illinois River below Meredosia, Ill. & 312 & 2,350 & 135 & 1,600 & $*$ & 4,560 & 5.6 \\
\hline $7-18$ & Mississippi River near Winfield, Mo. & 1,370 & 1,780 & 393 & 8,460 & * & 5,740 & 689 \\
\hline $7-20$ & Missouri River at Hermann, Mo. & 2,640 & 3,950 & 655 & 28,900 & * & 12,100 & 56 \\
\hline $7-21$ & Mississippi River at Hartford, 111. & 1,500 & 4,090 & 395 & 9,130 & * & 7,230 & 58 \\
\hline $7-22$ & Mississippi River at St. Louis, Mo. & 3,940 & 8,590 & 932 & 36,300 & * & 15,300 & 57 \\
\hline 7.23 & Mississippi River at Chester, Ill. & 4,250 & 12,200 & 1,010 & 39,000 & * & 16,000 & 64 \\
\hline $7-26$ & $\begin{array}{l}\text { Ohio River below Smithland Locks and Dam, } \\
\text { Ill.-Ky. }\end{array}$ & 652 & 495 & 74 & 3,010 & * & 3,820 & * \\
\hline $7-27$ & Ohio River at Olmsted, Ill. & 2,070 & 1,740 & 236 & 6,910 & * & 8,120 & * \\
\hline $7-28$ & Mississippi River below Hickman, Ky. & 6,270 & 5,410 & 1,790 & 52,500 & 27 & 51,000 & 186 \\
\hline 7.30 & Mississippi River at Helena, Ark. & 6,850 & 6,440 & 1,210 & 45,200 & * & 33,100 & 321 \\
\hline $7-31$ & White River at Mile 11.5, Ark. & 332 & 222 & 30 & 1,160 & 2.8 & 393 & 8.2 \\
\hline 8-01 & Arkansas River at Mile 55.9, Ark. & 790 & 410 & 431 & 8,000 & 14 & 5,940 & 39 \\
\hline 8-02 & Mississippi River above Arkansas City, Ark. & 7,630 & 2,650 & 1,940 & 51,800 & 125 & 42,600 & 627 \\
\hline 8-04 & Mississippi River below Vicksburg, Miss. & 7,750 & 3,210 & 1,560 & 56,000 & * & 33,500 & 325 \\
\hline $8-06$ & $\begin{array}{l}\text { Old River Outflow Channel near Knox } \\
\text { Landing, La. }\end{array}$ & 2,050 & 357 & 441 & 13,000 & 33 & 10,700 & 231 \\
\hline 8-07 & Mississippi River near St. Francisville, La. & 6,190 & 2,970 & 1,150 & 39,800 & * & 25,300 & 331 \\
\hline
\end{tabular}

Table 46.- Discharge and corresponding transport data for dissolved trace elements in water samples collected from the Mississippi River and some of its tributaries during the July-August 1987 cruise - Continued

\begin{tabular}{|c|c|c|c|c|c|c|c|c|}
\hline $\begin{array}{l}\text { Date } \\
1987\end{array}$ & Sampling site & Chromium & Cobalt & Copper & Iron & Lead & Lithium & Manganese \\
\hline $7-17$ & Illinois River below Meredosia, Ill. & 28 & $*$ & $*$ & 3,020 & 20 & 204 & 243 \\
\hline $7-18$ & Mississippi River near Winfield, Mo. & 136 & 2.6 & 338 & 2,140 & 132 & 936 & 54 \\
\hline $7-20$ & Missouri River at Hermann, Mo. & 329 & * & 895 & 26,600 & 178 & 4,200 & 38 \\
\hline $7-21$ & Mississippi River at Hartford, Ill. & 136 & * & 371 & $*$ & 102 & 871 & 111 \\
\hline $7-22$ & Mississippi River at St. Louis, Mo. & 314 & * & 766 & $*$ & 186 & 4,250 & 169 \\
\hline $7-23$ & Mississippi River at Chester, IIl. & 333 & $*$ & 852 & 5,600 & 241 & 4,280 & 195 \\
\hline $7-26$ & $\begin{array}{l}\text { Ohio River below Smithland Locks and Dam, } \\
\text { Ill.-Ky. }\end{array}$ & 17 & * & 99 & - & 17 & 262 & 20 \\
\hline $7-27$ & Ohio River at Olmsted, III. & 74 & * & 554 & $*$ & 50 & 577 & * \\
\hline $7-28$ & Mississippi River below Hickman, Ky. & 663 & 44 & 2,080 & * & 4,670 & 7,400 & * \\
\hline $7-30$ & Mississippi River at Helena, Ark. & 218 & $*$ & 1,180 & 3,460 & 2,670 & 4,870 & * \\
\hline $7-31$ & White River at Mile 11.5, Ark. & 15 & 4.1 & 36 & $*$ & 8 & 18 & * \\
\hline $8-01$ & Arkansas River at Mile 55.9, Ark. & 220 & 28 & 279 & $*$ & 25 & 658 & 67 \\
\hline $8-02$ & Mississippi River above Arkansas City, Ark. & 668 & 156 & 2,220 & * & 321 & 6,900 & 644 \\
\hline 8-04 & Mississippi River below Vicksburg, Miss. & 400 & * & 1,390 & * & 342 & 5,550 & * \\
\hline 8-06 & $\begin{array}{l}\text { Old River Outflow Channel near Knox } \\
\text { Landing, La. }\end{array}$ & 133 & 43 & 560 & $*$ & 92 & 1,640 & 143 \\
\hline $8-07$ & Mississippi River near St. Francisville, La. & * & * & 1,230 & * & 127 & 4,630 & 318 \\
\hline
\end{tabular}


Table 46.-- Discharge and corresponding transport data for dissolved trace elements in water samples collected from the Mississippi River and some of its tributaries during the July-August 1987 cruise - Continued

\begin{tabular}{|c|c|c|c|c|c|c|c|c|}
\hline $\begin{array}{l}\text { Date } \\
1987\end{array}$ & Sampling site & Molybdenum & Nickel & Strontium & Thallium & Uranium & Vanadium & Zinc \\
\hline 7.17 & Illinois River below Meredosia, III. & 213 & $*$ & 5,600 & 11 & * & 214 & $*$ \\
\hline $7-18$ & Mississippi River near Winfield, Mo. & 267 & 66 & 14,300 & 19 & 237 & 387 & 1,570 \\
\hline $7-20$ & Missouri River at Hermann, Mo. & 702 & $*$ & 61,200 & 40 & 503 & 549 & 786 \\
\hline $7-21$ & Mississippi River at Hartford, Ill. & 373 & $*$ & 16,800 & 24 & 214 & 329 & 295 \\
\hline $7-22$ & Mississippi River at St. Louis, Mo. & 1,080 & * & 76,400 & 66 & 725 & 623 & 331 \\
\hline $7-23$ & Mississippi River at Chester, Ill. & 1,100 & * & 79,000 & 74 & 770 & 689 & 560 \\
\hline $7-26$ & $\begin{array}{l}\text { Ohio River below Smithland Locks and Dam, } \\
\text { Ill.-Ky. }\end{array}$ & 188 & 24 & - & * & 25 & 32 & 120 \\
\hline $7-27$ & Ohio River at Olmsted, Ill. & 459 & * & 30,500 & * & 40 & 130 & 282 \\
\hline $7-28$ & Mississippi River below Hickman, Ky. & 2,190 & 3,300 & 120,000 & 260 & 1,470 & * & 981 \\
\hline $7-30$ & Mississippi River at Helena, Ark. & 1,590 & $*$ & 120,000 & * & 715 & 1,260 & 1,650 \\
\hline $7-31$ & White River at Mile 11.5, Ark. & 38 & * & 1,220 & * & 22 & * & 20 \\
\hline 8-01 & Arkansas River at Mile 55.9, Ark. & 133 & 53 & 31,800 & * & 144 & 1,010 & 122 \\
\hline 8-02 & Mississippi River above Arkansas City, Ark. & 2,060 & $*$ & 153,000 & $*$ & 1,260 & $*$ & 639 \\
\hline $8-04$ & Mississippi River below Vicksburg, Miss. & 1,580 & $*$ & 156,000 & * & 956 & 1,460 & 2,110 \\
\hline $8-06$ & $\begin{array}{l}\text { Old River Outflow Channel near Knox } \\
\text { Landing, La. }\end{array}$ & 535 & 163 & 40,100 & * & $*$ & * & 873 \\
\hline 8-07 & Mississippi River near St. Francisville, La. & 1,860 & * & 124,000 & 78 & * & * & 2,200 \\
\hline
\end{tabular}

Table 47.-- Discharge and corresponding transport data for dissolved trace elements in water samples collected from the Mississippi River and some of its tributaries during the NovemberDecember 1987 cruise

[*, concentration less than the detection limit; All results are in kilograms per day except discharge, which is in cubic meters per second ]

\begin{tabular}{|c|c|c|c|c|c|c|c|c|}
\hline $\begin{array}{l}\text { Date } \\
1987\end{array}$ & Sampling site & Discharae & Aluminum & Arsenic & Barium & Bervllium & Boron & Cadmium \\
\hline $11-29$ & Illinois River below Meredosia, Ill. & 262 & 152 & 62 & 1,050 & $*$ & 3,120 & 2.5 \\
\hline $11-30$ & Mississippi River near Winfield, Mo. & 2,040 & 1,330 & 211 & 8,070 & * & 5,180 & * \\
\hline $12-02$ & Missouri River at St. Charles, Mo. & 2,810 & 1,190 & 411 & 24,600 & * & 17,300 & * \\
\hline $12-03$ & Mississippi River at St. Louis, Mo. & 5,440 & 4,700 & 787 & 34,600 & * & 28,600 & * \\
\hline $12-05$ & Mississippi River at Thebes, III. & 5,190 & 12,100 & 749 & 35,200 & * & 23,200 & * \\
\hline $12-06$ & Ohio River at Olmsted, Ill. & 4,200 & 14,000 & 460 & 14,800 & * & 18,900 & * \\
\hline $12-07$ & Mississippi River below Hickman, Ky. & 8,820 & 5,510 & 914 & 47,200 & * & 44,600 & $*$ \\
\hline $12-08$ & Mississippi River at Fulton, Tenn. & 9,470 & 2,390 & 1,110 & 54,300 & * & 48,300 & * \\
\hline $12-11$ & Mississippi River at Helena, Ark. & $\mathbf{8 , 7 7 0}$ & 10,800 & 669 & 50,300 & * & 42,700 & * \\
\hline $12-12$ & White River at Mile 11.5, Ark. & 519 & 532 & * & 1,800 & * & 369 & * \\
\hline $12-13$ & Mississippi River above Arkansas City, Ark. & 9,920 & * & 1,460 & 61,100 & * & 42,900 & * \\
\hline $12-14$ & Yazoo River at Mile 10, Miss. & 177 & 662 & 16 & 466 & 1.0 & 270 & 9.7 \\
\hline $12-15$ & Mississippi River below Vicksburg, Miss. & 10,410 & 52,200 & 1,170 & 64,400 & * & 47,500 & 110 \\
\hline $12-17$ & $\begin{array}{l}\text { Old River Outflow Channel near Knox } \\
\text { Landing, La. }\end{array}$ & 1,830 & 835 & 221 & 11,500 & * & 8,370 & * \\
\hline $12-18$ & Mississippi River near St. Francisville, La. & 8,180 & 8,060 & 992 & 51,100 & * & 36,400 & * \\
\hline $12-20$ & Mississippi River below Belle Chasse, La. & 9,560 & 15,700 & 1,260 & 60,200 & * & 41,400 & * \\
\hline
\end{tabular}


Table 47.- Discharge and corresponding transport data for dissolved trace elements in water samples collected from the Mississippi River and some of its tributaries during the November-

December 1987 cruise - Continued

\begin{tabular}{|c|c|c|c|c|c|c|c|c|}
\hline $\begin{array}{l}\text { Date } \\
1987\end{array}$ & Sampling site & Chromium & Cobait & Copper & Iron & Lead & Llthium & Manganese \\
\hline 11.29 & Illinois River below Meredosia, Ill. & 12 & * & 42 & 138 & 6.7 & 157 & 9.3 \\
\hline $11-30$ & Mississippi River near Winfield, Mo. & 67 & * & 118 & 1,730 & 18 & 722 & 285 \\
\hline $12-02$ & Missouri River at St. Charles, Mo. & * & * & 265 & * & $*$ & 6,010 & 181 \\
\hline $12-03$ & Mississippi River at St. Louis, Mo. & 346 & * & 593 & 5,380 & 43 & 6,970 & 249 \\
\hline $12-05$ & Mississippi River at Thebes, Ill. & 184 & $*$ & 483 & 6,570 & 105 & 6,520 & 718 \\
\hline $12-06$ & Ohio River at Olmsted, Ill. & 222 & $*$ & 562 & 14,300 & 47 & 2,180 & 2,260 \\
\hline $12-07$ & Mississippi River below Hickman, Ky. & 367 & $*$ & 798 & * & $*$ & 6,940 & 555 \\
\hline $12-08$ & Mississippi River at Fulton, Tenn. & 308 & * & 827 & * & 87 & 8,130 & 125 \\
\hline $12-11$ & Mississippi River at Helena, Ark. & * & * & 455 & 8,680 & * & 7,270 & 863 \\
\hline $12-12$ & White River at Mile 11.5, Ark. & * & $*$ & 26 & 572 & 13 & 14 & 50 \\
\hline $12-13$ & Mississippi River above Arkansas City, Ark. & 6,940 & * & 855 & * & * & 7,310 & $*$ \\
\hline $12-14$ & Yazoo River at Mile 10, Miss. & 8.1 & 0.4 & 24 & 1,210 & 3.5 & 12 & 483 \\
\hline $12-15$ & Mississippi River below Vicksburg, Miss. & 697 & $*$ & 1,380 & 54,500 & 187 & 6,950 & 2,930 \\
\hline $12-17$ & $\begin{array}{l}\text { Old River Outflow Channel near Knox } \\
\text { Landing, La. }\end{array}$ & 104 & $*$ & 256 & $*$ & 26 & 1,260 & 178 \\
\hline $12-18$ & Mississippi River near St. Francisville, La. & 412 & $*$ & 1,060 & * & 57 & 5,750 & 298 \\
\hline $12-20$ & Mississippi River below Belle Chasse, La. & 513 & * & 1,370 & 9,140 & 208 & 7,040 & 332 \\
\hline
\end{tabular}

Table 47.-- Discharge and corresponding transport data for dissolved trace elements in water samples collected from the Mississippi River and some of its tributaries during the November-

December 1987 cruise - Continued

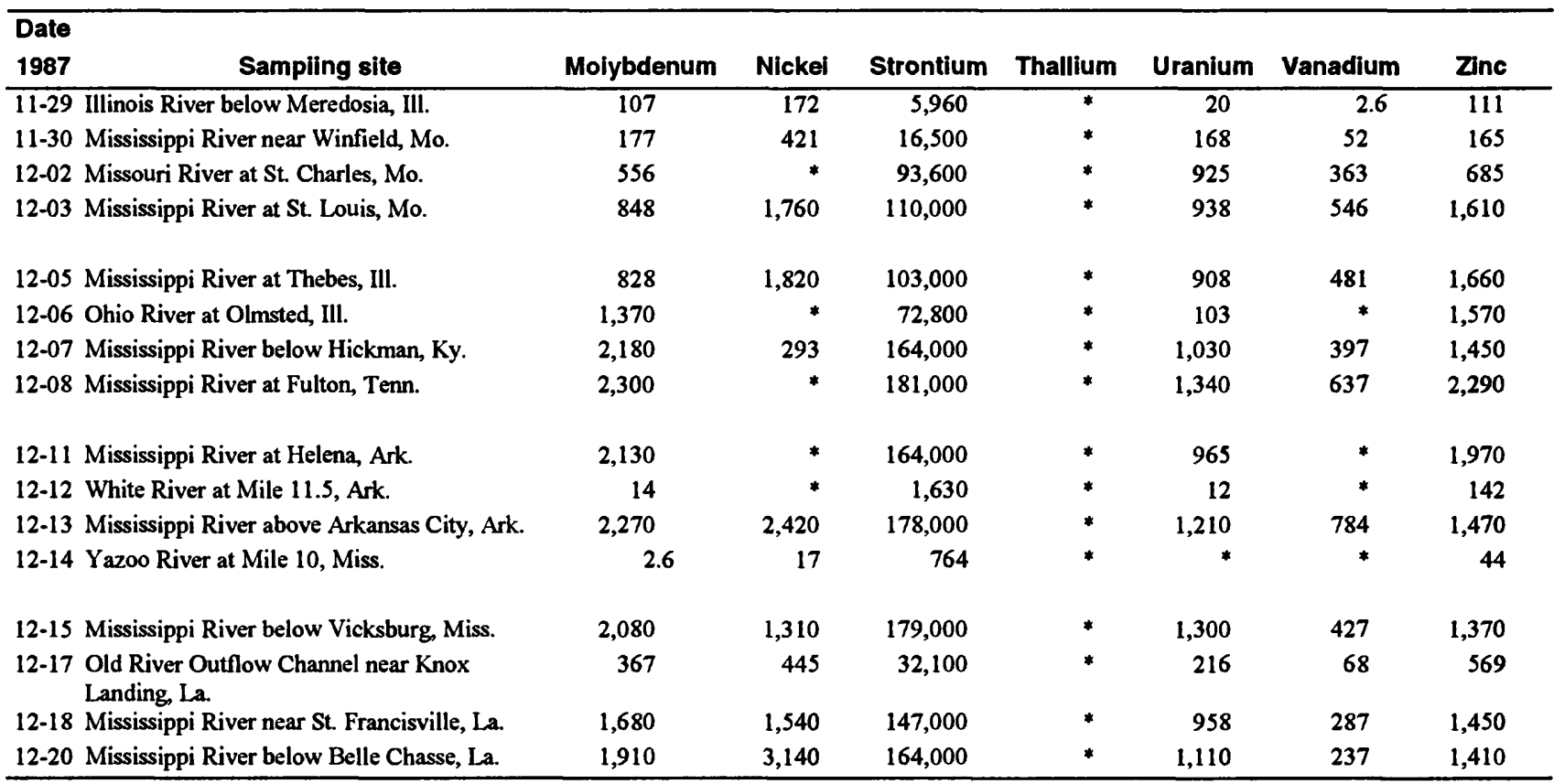


Table 48.- Discharge and corresponding transport data for dissolved trace elements in water samples collected from the Mississippi River and some of its tributaries during the May-June 1988 cruise

[ , concentration less than the detection limit; All results are in kilograms per day except discharge, which is in cubic meters per second ]

\begin{tabular}{|c|c|c|c|c|c|c|c|c|}
\hline $\begin{array}{l}\text { Date } \\
1988\end{array}$ & Sampling site & Discharge & Aluminum & Arsenic & Barium & Beryllium & Boron & Cadmium \\
\hline $5-16$ & Illinois River below Meredosia, Ill. & 332 & 229 & 64 & 1,540 & 4.1 & 3,500 & 12 \\
\hline 5-17 & Mississippi River near Winfield, Mo. & 1,740 & 831 & 248 & 7,550 & 19 & 4,270 & 55 \\
\hline 5-19 & Missouri River at Hermann, Mo. & 1,480 & 392 & 366 & 12,000 & 19 & 11,600 & 63 \\
\hline $5-20$ & Mississippi River at St. Louis, Mo. & 3,350 & 1,140 & 664 & 22,000 & 44 & 16,300 & 171 \\
\hline $5-22$ & Mississippi River at Thebes, Ill. & 3,590 & * & 406 & 22,700 & * & 15,800 & * \\
\hline $5-23$ & Ohio River at Olmsted, Ill. & 3,230 & 1,840 & 201 & 10,700 & 17 & 10,800 & * \\
\hline $5-24$ & Mississippi River below Hickman, Ky. & 6,790 & 2,340 & 820 & 37,200 & 36 & 29,700 & * \\
\hline $5-26$ & Mississippi River at Fulton, Tenn. & 7,170 & 1,880 & 924 & 38,900 & 365 & 30,000 & 349 \\
\hline 5-28 & Mississippi River at Helena, Ark. & 7,050 & 81,400 & 1,010 & 40,100 & 366 & 28,200 & 393 \\
\hline 5-29 & White River at Mile 11.5, Ark. & 438 & 116 & 34 & 1,430 & 22 & 421 & 25 \\
\hline $5-29$ & Arkansas River at Mile 1.0, Ark. & 8,160 & 11,100 & 998 & 44,800 & 262 & 32,500 & 230 \\
\hline $5-30$ & Mississippi River above Arkansas City, Ark. & 73 & 40 & 8.1 & 479 & 2.3 & 113 & 1.1 \\
\hline $6-02$ & Mississippi River below Vicksburg, Miss. & 7,950 & 4,130 & 863 & 41,500 & 248 & 28,600 & 142 \\
\hline 6-04 & $\begin{array}{l}\text { Old River Outflow Channel near Knox } \\
\text { Landing, La. }\end{array}$ & 2,150 & 553 & 157 & 11,500 & * & 6,910 & * \\
\hline $6-05$ & Mississippi River near St. Francisville, La. & 5,700 & 721 & 467 & 32,600 & * & 19,800 & $*$ \\
\hline $6-07$ & Mississippi River below Belle Chasse, La. & 5,570 & 6,920 & 522 & 34,000 & * & 17,400 & * \\
\hline
\end{tabular}

Table 48.-- Discharge and corresponding transport data for dissolved trace elements in water samples collected from the Mississippi River and some of its tributaries during the May-June 1988 cruise - Continued

\begin{tabular}{|c|c|c|c|c|c|c|c|c|}
\hline $\begin{array}{l}\text { Date } \\
1988\end{array}$ & Sampling site & Chromium & Cobalt & Copper & Iron & Lead & Lithium & Manganese \\
\hline $5-16$ & Illinois River below Meredosia, Ill. & 13 & 16 & 67 & * & 8.5 & 186 & 21 \\
\hline $5-17$ & Mississippi River near Winfield, Mo. & 85 & 42 & 198 & * & 26 & 880 & 855 \\
\hline 5-19 & Missouri River at Hermann, Mo. & 73 & * & 242 & * & 32 & 4,650 & 95 \\
\hline $5-20$ & Mississippi River at St. Louis, Mo. & 311 & 77 & 464 & * & 105 & 5,520 & 259 \\
\hline 5-22 & Mississippi River at Thebes, $\mathrm{Ill}$. & * & * & 29 & * & * & 4,890 & * \\
\hline $5-23$ & Ohio River at Olmsted, Ill. & * & * & 307 & * & * & 1,310 & 101 \\
\hline $5-24$ & Mississippi River below Hickman, Ky. & * & * & 658 & * & * & 7,350 & 275 \\
\hline $5-26$ & Mississippi River at Fulton, Tenn. & 574 & 7 & 1,370 & * & 68 & 7,310 & 839 \\
\hline $5-28$ & Mississippi River at Helena, Ark. & 759 & 27 & 1,140 & * & 190 & 6,580 & 26,900 \\
\hline $5-29$ & White River at Mile 11.5, Ark. & 65 & 17 & 25 & * & 4.1 & 34 & 221 \\
\hline $5-29$ & Arkansas River at Mile 1.0, Ark. & 600 & 4 & 1,060 & 20,100 & 291 & 7,340 & 2,490 \\
\hline $5-30$ & Mississippi River above Arkansas City, Ark. & 9.6 & * & 7.1 & * & 2.4 & 17 & 636 \\
\hline $6-02$ & Mississippi River below Vicksburg, Miss. & 945 & * & 999 & * & 247 & 6,500 & 304 \\
\hline 6-04 & $\begin{array}{l}\text { Old River Outflow Channel near Knox } \\
\text { Landing, La. }\end{array}$ & $*$ & * & 13 & * & $*$ & 1,690 & $*$ \\
\hline $6-05$ & Mississippi River near St. Francisville, La. & * & * & 19 & * & * & 4,240 & * \\
\hline 6-07 & Mississippi River below Belle Chasse, La. & * & * & 193 & * & * & 4,410 & 115 \\
\hline
\end{tabular}


Table 48.-- Discharge and corresponding transport data for dissolved trace elements in water samples collected from the Mississippi River and some of its tributaries during the May-June 1988 cruise - Continued

\begin{tabular}{|c|c|c|c|c|c|c|c|c|}
\hline $\begin{array}{l}\text { Date } \\
1988\end{array}$ & Sampling site & Molybdenum & Nickel & Strontium & Thallium & Uranium & Vanadium & Zinc \\
\hline $5-16$ & Illinois River below Meredosia, III. & 165 & 234 & 5,590 & $*$ & 36 & 40 & 106 \\
\hline $5-17$ & Mississippi River near Winfield, Mo. & 189 & 284 & 15,100 & * & 209 & 175 & 149 \\
\hline $5-19$ & Missouri River at Hermann, Mo. & 395 & $*$ & 59,600 & * & 440 & 354 & 190 \\
\hline $5-20$ & Mississippi River at St. Louis, Mo. & 792 & 571 & 76,500 & $*$ & 428 & 524 & 335 \\
\hline $5-22$ & Mississippi River at Thebes, Ill. & 716 & 481 & 85,600 & $*$ & 938 & 407 & * \\
\hline $5-23$ & Ohio River at Olmsted, Ill. & 769 & $*$ & 54,800 & 106 & 259 & * & 121 \\
\hline $5-24$ & Mississippi River below Hickman, Ky. & 1,700 & * & 140,000 & 218 & 1,340 & * & * \\
\hline $5-26$ & Mississippi River at Fulton, Tenn. & 1,600 & 1,680 & 138,000 & * & 1,270 & * & 961 \\
\hline $5-28$ & Mississippi River at Helena, Ark. & 1,500 & 1,250 & 131,000 & $*$ & 1,270 & * & 1,650 \\
\hline $5-29$ & White River at Mile 11.5, Ark. & 35 & 35 & 1,520 & $*$ & 23 & * & 54 \\
\hline $5-29$ & Arkansas River at Mile 1.0, Ark. & 1,860 & 2,990 & 144,000 & 99 & 1,400 & * & 952 \\
\hline $5-30$ & Mississippi River above Arkansas City, Ark. & 8.1 & $*$ & 766 & 0.8 & 4.3 & * & 7.6 \\
\hline $6-02$ & Mississippi River below Vicksburg, Miss. & 1,670 & $*$ & 149,000 & 92 & 1,320 & $*$ & 1,170 \\
\hline $6-04$ & $\begin{array}{l}\text { Old River Outflow Channel near Knox } \\
\text { Landing, La. }\end{array}$ & 413 & 643 & 40,000 & $*$ & 301 & 118 & $*$ \\
\hline $6-05$ & Mississippi River near St. Francisville, La. & 1,090 & 818 & 110,000 & * & 775 & 789 & * \\
\hline $6-07$ & Mississippi River below Belle Chasse, La. & 993 & 1,960 & 109,000 & $*$ & 849 & 400 & $*$ \\
\hline
\end{tabular}

Table 49.-- Discharge and corresponding transport data for dissolved trace elements in water samples collected from the Mississippi River and some of its tributaries during the March-April 1989 cruise

[ *, concentration less than the detection limit; All results are in kilograms per day except discharge, which is in cubic meters per second ]

\begin{tabular}{|c|c|c|c|c|c|c|c|c|}
\hline $\begin{array}{l}\text { Date } \\
1989\end{array}$ & Sampling site & Discharge & Aluminum & Arsenic & Barium & Beryllium & Boron & Cadmium \\
\hline 3-09 & Illinois River at Hardin, III. & 410 & 152 & 72 & 1,790 & $*$ & 4,780 & $*$ \\
\hline $3-10$ & Mississippi River near Winfield, Mo. & 850 & 260 & 70 & 3,340 & $*$ & 2,740 & $*$ \\
\hline $3-12$ & Missouri River at Hermann, Mo. & 1,480 & 1,130 & 217 & 8,340 & $*$ & 6,470 & $*$ \\
\hline $3-13$ & Mississippi River at St. Louis, Mo. & 3,940 & 1,240 & 522 & 18,400 & $*$ & 20,700 & $*$ \\
\hline $3-15$ & Mississippi River at Thebes, III. & 4,890 & 1,110 & 432 & 24,900 & $*$ & 23,600 & $*$ \\
\hline $3-16$ & Ohio River at Olmsted, Ill. & 20,400 & 15,000 & $*$ & 47,000 & $*$ & 28,300 & $*$ \\
\hline 3-17 & Mississippi River below Hickman, Ky. & 24,700 & 22,600 & * & 72,300 & $*$ & 48,700 & $*$ \\
\hline 3-19 & Mississippi River below Fulton, Tenn. & 24,800 & 12,900 & * & 77,700 & * & 52,800 & * \\
\hline $3-21$ & Mississippi River at Helena, Ark. & 25,900 & 373,000 & * & 93,200 & * & 52,900 & * \\
\hline 3-22 & White River at Mile 11.5, Ark. & 1,500 & 1,120 & 125 & 4,040 & * & 971 & * \\
\hline 3-23 & Arkansas River at Pendleton, Ark. & 1,900 & 1,010 & 153 & 8,100 & $*$ & 4,540 & * \\
\hline 3-24 & Mississippi River above Arkansas City, Ark. & 26,800 & 10,600 & 2,230 & 101,000 & * & 58,700 & * \\
\hline $3-26$ & Yazoo River below Steele Bayou, Miss. & 1,500 & 1,230 & * & 3,260 & $*$ & 1,610 & $*$ \\
\hline 3-27 & Mississippi River below Vicksburg, Miss. & 26,600 & 9,250 & 2,190 & 105,000 & * & 55,400 & $*$ \\
\hline $3-29$ & $\begin{array}{l}\text { Old River Outflow Channel near Knox } \\
\text { Landing, La. }\end{array}$ & 6,160 & 2,100 & 735 & 25,100 & * & 12,400 & * \\
\hline $3-30$ & Mississippi River near St. Francisville, La. & 23,100 & 4,410 & 2,260 & 95,500 & * & 45,600 & * \\
\hline 4-01 & Mississippi River below Belle Chasse, La. & 22,500 & 6,300 & 1,880 & 95,400 & * & 44,200 & * \\
\hline
\end{tabular}


Table 49.-- Discharge and corresponding transport data for dissolved trace elements in water samples collected from the Mississippi River and some of its tributaries during the March-April 1989 cruise - Continued

\begin{tabular}{|c|c|c|c|c|c|c|c|c|}
\hline $\begin{array}{l}\text { Date } \\
1989\end{array}$ & Sampling site & Chromium & Cobalt & Copper & Iron & Lead & Lithlum & Manganese \\
\hline $3-09$ & Illinois River at Hardin, III. & 34 & 30 & 77 & $*$ & 9 & 229 & 603 \\
\hline $3-10$ & Mississippi River near Winfield, Mo. & 53 & 34 & 89 & 1,220 & 8.8 & 240 & 130 \\
\hline $3-12$ & Missouri River at Hermann, Mo. & 48 & 44 & 130 & 1,130 & 15 & 1,910 & 568 \\
\hline $3-13$ & Mississippi River at St. Louis, Mo. & 86 & 153 & 429 & * & 24 & 2,760 & 999 \\
\hline $3-15$ & Mississippi River at Thebes, Ill. & $*$ & 180 & 540 & $*$ & 58 & 3,340 & 555 \\
\hline $3-16$ & Ohio River at Olmsted, Ill. & $*$ & 361 & 1,180 & 12,300 & $*$ & 2,970 & 6,990 \\
\hline $3-17$ & Mississippi River below Hickman, Ky. & $*$ & 489 & 1,560 & $*$ & $*$ & 5,640 & 3,610 \\
\hline 3-19 & Mississippi River below Fulton, Tenn. & $*$ & 499 & 1,580 & $*$ & $*$ & 5,750 & 3,590 \\
\hline $3-21$ & Mississippi River at Helena, Ark. & $*$ & 743 & 1,940 & - & $*$ & 6,080 & 25,000 \\
\hline $3-22$ & White River at Mile 11.5, Ark. & $*$ & 13 & 41 & 1,780 & * & 29 & 416 \\
\hline $3-23$ & Arkansas River at Pendleton, Ark. & $*$ & 26 & 82 & 1,520 & $*$ & 362 & 313 \\
\hline $3-24$ & Mississippi River above Arkansas City, Ark. & $*$ & 492 & 1,130 & $*$ & $*$ & 6,800 & 3,560 \\
\hline 3-26 & Yazoo River below Steele Bayou, Miss. & $*$ & 4 & 90 & 4,160 & * & 110 & 1,220 \\
\hline 3-27 & Mississippi River below Vicksburg, Miss. & $*$ & 443 & 1,120 & * & * & 6,710 & 1,710 \\
\hline $3-29$ & $\begin{array}{l}\text { Old River Outflow Channel near Knox } \\
\text { Landing, La. }\end{array}$ & $*$ & 90 & 494 & $*$ & * & 1,620 & 1,350 \\
\hline $3-30$ & Mississippi River near St. Francisville, La. & $*$ & 326 & 1,740 & $*$ & * & 6,190 & 3,290 \\
\hline $4-01$ & Mississippi River below Belle Chasse, La. & $*$ & 255 & 1,790 & $*$ & * & 5,870 & 4,230 \\
\hline
\end{tabular}

Table 49.- Discharge and corresponding transport data for dissolved trace elements in water samples collected from the Mississippi River and some of its tributaries during the March-April 1989 cruise -- Continued

\begin{tabular}{|c|c|c|c|c|c|c|c|c|}
\hline $\begin{array}{l}\text { Date } \\
1989\end{array}$ & Sampling site & Molybdenum & Nickel & Strontium & Thallium & Uranlum & Vanadlum & Zinc \\
\hline $3-09$ & Illinois River at Hardin, Ill. & 225 & 680 & 9,790 & * & 57 & 62 & 296 \\
\hline $3-10$ & Mississippi River near Winfield, Mo. & 95 & 679 & 6,830 & * & 58 & 50 & 212 \\
\hline $3-12$ & Missouri River at Hermann, Mo. & 226 & 959 & 34,200 & * & 407 & 157 & 204 \\
\hline $3-13$ & Mississippi River at St. Louis, Mo. & 815 & 3,120 & 66,600 & * & 672 & 333 & 729 \\
\hline $3-15$ & Mississippi River at Thebes, Ill. & 898 & 3,510 & 81,100 & * & 747 & 480 & 830 \\
\hline $3-16$ & Ohio River at Olmsted, Ill. & 1,350 & 8,340 & 189,000 & * & 303 & 580 & 438 \\
\hline $3-17$ & Mississippi River below Hickman, Ky. & 2,170 & 11,400 & 258,000 & * & 963 & 932 & 998 \\
\hline $3-19$ & Mississippi River below Fulton, Tenn. & 2,000 & 11,700 & 254,000 & * & 1,010 & 856 & 1,310 \\
\hline $3-21$ & Mississippi River at Helena, Ark. & 2,020 & 12,700 & 271,000 & * & 1,130 & 1,650 & 1,940 \\
\hline $3-22$ & White River at Mile 11.5, Ark. & 40 & 400 & 3,520 & $*$ & * & 44 & 77 \\
\hline $3-23$ & Arkansas River at Pendleton, Ark. & 93 & 683 & 27,500 & * & 79 & 76 & 108 \\
\hline $3-24$ & Mississippi River above Arkansas City, Ark. & 2,250 & 12,500 & 283,000 & * & 1,120 & 1,150 & 8,590 \\
\hline $3-26$ & Yazoo River below Steele Bayou, Miss. & 20 & 307 & 4,670 & $*$ & * & 68 & 87 \\
\hline $3-27$ & Mississippi River below Vicksburg, Miss. & 2,160 & 11,700 & 280,000 & * & 1,160 & 1,210 & 1,120 \\
\hline $3-29$ & $\begin{array}{l}\text { Old River Outflow Channel near Knox } \\
\text { Landing, La. }\end{array}$ & 540 & 2,850 & 64,300 & $*$ & 359 & 379 & 2,000 \\
\hline $3-30$ & Mississippi River near St. Francisville, La. & 1,960 & 10,800 & 242,000 & $*$ & 1,410 & 1,400 & 1,720 \\
\hline 4-01 & Mississippi River below Belle Chasse, La. & 2,020 & 9,800 & 235,000 & $*$ & 1,500 & 1,710 & 3,770 \\
\hline
\end{tabular}


Table 50.-- Discharge and corresponding transport data for dissolved trace elements in water samples collected from the Mississippi River and some of its tributaries during the June 1989 cruise

[ ", concentration less than the detection limit; All results are in kilograms per day except discharge, which is in cubic meters per second ]

\begin{tabular}{|c|c|c|c|c|c|c|c|c|}
\hline $\begin{array}{l}\text { Date } \\
1989\end{array}$ & Sampling site & Discharge & Aluminum & Arsenic & Barium & Beryilium & Boron & Cadmium \\
\hline$\overline{6-04}$ & Illinois River at Hardin, Ill. & 780 & 488 & 212 & 4,460 & 2.9 & 10,400 & $*$ \\
\hline $6-05$ & Mississippi River near Winfield, Mo. & 2,320 & 938 & 458 & 12,600 & $*$ & $\mathbf{8 , 8 8 0}$ & * \\
\hline $6-07$ & Missouri River at Hermann, Mo. & 1,760 & 906 & 399 & 13,300 & * & 16,800 & * \\
\hline $6-08$ & Mississippi River at St. Louis, Mo. & 4,760 & 1,430 & 835 & 30,900 & $*$ & 37,500 & $*$ \\
\hline $6-10$ & Mississippi River at Thebes, Ill. & 5,230 & 1,800 & 1,140 & 34,300 & 15 & 43,200 & $*$ \\
\hline $6-11$ & Ohio River at Olmsted, Ill. & 8,760 & 4,520 & 925 & 29,500 & * & 24,700 & * \\
\hline 6-12 & Mississippi River below Hickman, Ky. & 14,100 & 7,220 & 1,810 & 64,400 & * & 57,200 & * \\
\hline $6-14$ & Mississippi River at Fulton, Tenn. & 15,300 & 7,940 & 1,810 & 72,200 & * & 63,600 & * \\
\hline 6-17 & Mississippi River at Helena, Ark. & 16,900 & 11,600 & 2,000 & 75,800 & * & 63,700 & * \\
\hline 6-18 & White River at Mile 11.5, Ark. & 770 & 1,480 & 66 & 2,910 & * & 614 & $*$ \\
\hline 6-19 & Arkansas River at Pendleton, Ark. & 3,600 & 20,400 & 554 & 23,000 & $*$ & 13,500 & $*$ \\
\hline $6-20$ & Mississippi River above Arkansas City, Ark. & 23,300 & 145,000 & 3,020 & 102,000 & * & 69,400 & * \\
\hline 6-22 & Yazoo River below Steele Bayou, Miss. & 1,070 & 2,210 & 150 & 2,350 & * & 1,380 & * \\
\hline $6-23$ & Mississippi River below Vicksburg, Miss. & 24,800 & 9,600 & 2,660 & 103,000 & * & 66,000 & * \\
\hline $6-25$ & $\begin{array}{l}\text { Old River Outflow Channel near Knox } \\
\text { Landing, La. }\end{array}$ & 4,890 & 1,880 & 518 & 20,400 & * & 13,300 & * \\
\hline 6-26 & Mississippi River near St. Francisville, La. & 19,000 & 6,080 & 2,430 & 80,200 & * & 55,500 & $*$ \\
\hline 6-28 & Mississippi River below Belle Chasse, La. & 20,100 & 7,820 & 3,490 & 86,700 & * & 60,200 & * \\
\hline
\end{tabular}

Table 50.- Discharge and corresponding transport data for dissolved trace elements in water samples collected from the Mississippi River and some of its tributaries during the June 1989 cruise - Continued

\begin{tabular}{|c|c|c|c|c|c|c|c|c|}
\hline $\begin{array}{l}\text { Date } \\
1989\end{array}$ & Sampling site & Chromium & Cobait & Copper & Iron & Lead & Lithium & Manganese \\
\hline $6-04$ & Illinois River at Hardin, Ill. & 38 & 49 & 145 & * & 5.8 & 427 & 24 \\
\hline $6-05$ & Mississippi River near Winfield, Mo. & 79 & 84 & 287 & * & $*$ & 947 & 84 \\
\hline $6-07$ & Missouri River at Hermann, Mo. & 85 & 55 & 235 & * & * & 4,970 & 37 \\
\hline $6-08$ & Mississippi River at St. Louis, Mo. & 149 & 173 & 625 & * & * & 6,910 & 176 \\
\hline $6-10$ & Mississippi River at Thebes, Ill. & 176 & 189 & 727 & * & * & 7,270 & 265 \\
\hline $6-11$ & Ohio River at Olmsted, Ill. & * & 88 & 927 & * & * & 1,590 & 749 \\
\hline $6-12$ & Mississippi River below Hickman, Ky. & * & 212 & 1,540 & * & * & 6,550 & 4,230 \\
\hline $6-14$ & Mississippi River at Fulton, Tenn. & * & 202 & 1,600 & * & * & 6,930 & 4,040 \\
\hline $6-17$ & Mississippi River at Helena, Ark. & * & 178 & 1,780 & * & * & 6,480 & 237 \\
\hline $6-18$ & White River at Mile 11.5, Ark. & * & 2.2 & 36 & 1,770 & * & 20 & 111 \\
\hline $6-19$ & Arkansas River at Pendleton, Ark. & * & 84 & 539 & 19,000 & 53 & 1,060 & 1,050 \\
\hline $6-20$ & Mississippi River above Arkansas City, Ark. & * & 586 & 2,880 & 156,000 & 336 & 7,140 & 8,900 \\
\hline $6-22$ & Yazoo River below Steele Bayou, Miss. & * & 5.3 & 176 & 3,630 & * & 64 & 243 \\
\hline $6-23$ & Mississippi River below Vicksburg, Miss. & * & 447 & 2,940 & * & * & 6,220 & 710 \\
\hline $6-25$ & $\begin{array}{l}\text { Old River Outflow Channel near Knox } \\
\text { Landing, La. }\end{array}$ & * & 92 & 569 & * & * & 1,300 & 161 \\
\hline $6-26$ & Mississippi River near St. Francisville, La. & * & 427 & 2,480 & * & * & 5,550 & 465 \\
\hline $6-28$ & Mississippi River below Belle Chasse, La. & * & 423 & 3,580 & * & $*$ & 6,100 & 464 \\
\hline
\end{tabular}


Table 50.- Discharge and corresponding transport data for dissolved trace elements in water samples collected from the Mississippi River and some of its tributaries during the June 1989 cruise - Continued

\begin{tabular}{|c|c|c|c|c|c|c|c|c|}
\hline $\begin{array}{l}\text { Date } \\
1989\end{array}$ & Sampling site & Molybdenum & Nickel & Strontium & Thallium. & Uranium & Vanadium & Zinc \\
\hline 6-04 & Illinois River at Hardin, Ill. & 385 & 972 & 14,700 & 3.9 & 109 & 226 & 287 \\
\hline 6-05 & Mississippi River near Winfield, Mo. & 372 & 1,470 & 18,900 & $*$ & 273 & 442 & 654 \\
\hline 6-07 & Missouri River at Hermann, Mo. & 502 & 1,300 & 60,800 & $*$ & 650 & 404 & 824 \\
\hline 6-08 & Mississippi River at St. Louis, Mo. & 1,260 & 3,600 & 93,800 & * & 1,050 & 1,080 & 1,580 \\
\hline $6-10$ & Mississippi River at Thebes, Ill. & 1,410 & 3,950 & 97,000 & * & 1,060 & 1,200 & 1,400 \\
\hline $6-11$ & Ohio River at Olmsted, Iil. & 1,710 & 4,130 & 129,000 & 55 & 392 & 499 & 1,790 \\
\hline $6-12$ & Mississippi River below Hickman, Ky. & 2,910 & 7,870 & 218,000 & $*$ & 1,210 & 1,540 & 2,170 \\
\hline $6-14$ & Mississippi River at Fulton, Tenn. & 2,890 & 8,010 & 236,000 & * & 1,220 & 1,460 & 3,440 \\
\hline $6-17$ & Mississippi River at Helena, Ark. & 3,230 & 8,350 & 255,000 & * & 1,050 & 1,650 & 1,310 \\
\hline $6-18$ & White River at Mile 11.5, Ark. & 54 & 252 & 7,100 & * & 16 & 48 & 79 \\
\hline $6-19$ & Arkansas River at Pendleton, Ark. & 340 & 1,780 & 85,000 & 26 & 278 & 519 & 804 \\
\hline $6-20$ & Mississippi River above Arkansas City, Ark. & 3,530 & 11,500 & 374,000 & $*$ & 1,610 & 2,460 & 4,610 \\
\hline $6-22$ & Yazoo River below Steele Bayou, Miss. & 33 & 226 & 5,690 & * & * & 94 & 178 \\
\hline $6-23$ & Mississippi River below Vicksburg, Miss. & 3,610 & 10,400 & 337,000 & * & 1,260 & 2,180 & 8,130 \\
\hline 6-25 & $\begin{array}{l}\text { Old River Outflow Channel near Knox } \\
\text { Landing, La. }\end{array}$ & 707 & 2,210 & 63,100 & $*$ & 260 & 469 & 599 \\
\hline $6-26$ & Mississippi River near St. Francisville, La. & 3,150 & 8,690 & 254,000 & * & 1,090 & 2,150 & 3,280 \\
\hline $6-28$ & Mississippi River below Belle Chasse, La. & 3,190 & 9,290 & 239,000 & * & 1,220 & 2,400 & 2,920 \\
\hline
\end{tabular}

Table 51.- Discharge and corresponding transport data for dissolved trace elements in water samples collected from the Mississippi River and some of its tributaries during the FebruaryMarch 1990 cruise

[ *, concentration less than the detection limit; All results are in kilograms per day except discharge, which is in cubic meters per second ]

\begin{tabular}{|c|c|c|c|c|c|c|c|c|}
\hline $\begin{array}{l}\text { Date } \\
1990\end{array}$ & Sampling site & Discharge & Aluminum & Arsenic & Barium & Beryllium & Boron & Cadmlum \\
\hline $2-23$ & Cumberland River near Smithland, Ky. & 2,170 & 2,600 & $*$ & 4,250 & $*$ & 2,400 & $*$ \\
\hline $2-24$ & Tennessee River near Calvert City, Ky. & 6,570 & 15,200 & * & 11,300 & * & 4,140 & $*$ \\
\hline $2-25$ & Mississippi River near Cache, Ill. & 4,240 & 2,620 & 646 & 25,400 & * & 23,900 & * \\
\hline $2-28$ & Wabash River near New Haven, Ill. & 2,340 & 961 & * & 8,170 & * & 7,560 & * \\
\hline 3-01 & Ohio River at Uniontown, Ky. & 6,620 & 7,030 & * & 17,900 & * & 14,700 & * \\
\hline $3-03$ & Ohio River at Olmsted, Ill. & 16,100 & 8,700 & * & 39,400 & * & 29,700 & * \\
\hline 3-04 & Mississippi River below Hickman, Ky. & 21,000 & 11,200 & * & 66,800 & * & 45,700 & * \\
\hline 3-05 & Mississippi River below Fulton, Tenn. & 22,800 & 9,620 & * & 70,400 & * & 50,600 & * \\
\hline 3-07 & Mississippi River at Helena, Ark. & 23,330 & 8,070 & 2,580 & 82,700 & * & 48,100 & * \\
\hline 3-08 & Mississippi River above Arkansas City, Ark. & 33,200 & 9,110 & 2,430 & 115,000 & * & 64,000 & * \\
\hline 3-10 & Mississippi River below Vicksburg, Miss. & 34,100 & 11,500 & 2,970 & 118,000 & * & 63,000 & * \\
\hline $3-12$ & Mississippi River near St. Francisville, La. & 26,300 & 7,960 & 1,770 & 96,000 & * & 49,000 & $*$ \\
\hline $3-14$ & Mississippi River below Belle Chasse, La. & 26,700 & 7,380 & 3,090 & 101,000 & 87 & 51,000 & * \\
\hline
\end{tabular}


Table 51.- Discharge and corresponding transport data for dissolved trace elements in water samples collected from the Mississippi River and some of its tributaries during the FebruaryMarch 1990 cruise - Continued

\begin{tabular}{|c|c|c|c|c|c|c|c|c|}
\hline $\begin{array}{l}\text { Date } \\
1990\end{array}$ & Sampling site & Chromium & Cobalt & Copper & Iron & Lead & LIthium & Manganese \\
\hline $2-23$ & Cumberland River near Smithland, Ky. & * & 53 & 147 & 2,240 & * & 138 & 264 \\
\hline $2-24$ & Tennessee River near Calvert City, Ky. & $*$ & 107 & 419 & 10,900 & * & 345 & 619 \\
\hline $2-25$ & Mississippi River near Cache, Ill. & 228 & 195 & 711 & 2,590 & * & 3,360 & 512 \\
\hline $2-28$ & Wabash River near New Haven, III. & * & 102 & 345 & 1,120 & * & 308 & 711 \\
\hline 3-01 & Ohio River at Uniontown, Ky. & * & 180 & 861 & 6,920 & * & 1,560 & 3,420 \\
\hline 3-03 & Ohio River at Olmsted, III. & * & 303 & 1,790 & $*$ & * & 2,340 & 5,090 \\
\hline 3-04 & Mississippi River below Hickman, Ky. & * & 481 & 2,280 & * & 156 & 4,800 & 5,690 \\
\hline 3-05 & Mississippi River below Fulton, Tenn. & * & 534 & 2,780 & 58,600 & 223 & 5,080 & 6,150 \\
\hline 3-07 & Mississippi River at Helena, Ark. & * & 556 & 3,190 & 21,900 & * & 4,940 & 3,290 \\
\hline 3-08 & Mississippi River above Arkansas City, Ark. & * & 711 & 3,530 & 29,500 & * & 6,460 & 4,150 \\
\hline $3-10$ & Mississippi River below Vicksburg, Miss. & * & 672 & 4,320 & * & * & 6,350 & 3,190 \\
\hline $3-12$ & Mississippi River near St. Francisville, La. & * & 546 & 3,800 & * & * & 4,900 & 4,040 \\
\hline $3-14$ & Mississippi River below Belle Chasse, La. & * & 588 & 5,080 & * & * & 4,960 & 14,500 \\
\hline
\end{tabular}

Table 51.- Discharge and corresponding transport data for dissolved trace elements in water samples collected from the Mississippi River and some of its tributaries during the FebruaryMarch 1990 cruise - Continued

\begin{tabular}{|c|c|c|c|c|c|c|c|c|}
\hline $\begin{array}{l}\text { Date } \\
1990\end{array}$ & Sampling site & Molybdenum & Nickel & Strontium & Thallium & Uranium & Vanadium & Zinc \\
\hline $2-23$ & Cumberland River near Smithland, Ky. & 144 & 1,110 & 15,900 & $*$ & $*$ & 106 & 387 \\
\hline $2-24$ & Tennessee River near Calvert City, Ky. & 119 & 1,840 & 26,000 & $*$ & $*$ & 296 & 984 \\
\hline $2-25$ & Mississippi River near Cache, III. & 992 & 3,460 & 62,900 & $*$ & 754 & 571 & 1,160 \\
\hline $2-28$ & Wabash River near New Haven, III. & 347 & 1,930 & 22,200 & * & 151 & 100 & 324 \\
\hline $3-01$ & Ohio River at Uniontown, Ky. & 888 & 3,790 & 101,000 & $*$ & 131 & 205 & 1,390 \\
\hline $3-03$ & Ohio River at Olmsted, Ill. & 1,790 & 8,100 & 161,000 & * & 548 & 523 & 4,420 \\
\hline $3-04$ & Mississippi River below Hickman, Ky. & 2,470 & 11,100 & 234,000 & $*$ & 1,210 & 987 & 2,630 \\
\hline 3-05 & Mississippi River below Fulton, Tenn. & 2,480 & 11,900 & 237,000 & * & 1,260 & 1,300 & 4,360 \\
\hline 3-07 & Mississippi River at Helena, Ark. & 2,570 & 11,700 & 238,000 & $*$ & 1,340 & 1,450 & 4,560 \\
\hline 3-08 & Mississippi River above Arkansas City, Ark. & 2,830 & 14,700 & 357,000 & * & 1,840 & 1,550 & 4,000 \\
\hline $3-10$ & Mississippi River below Vicksburg, Miss. & 2,830 & 14,000 & 291,000 & * & 1,580 & 1,700 & 6,080 \\
\hline $3-12$ & Mississippi River near St. Francisville, La. & 2,190 & 11,700 & 248,000 & $*$ & 1,240 & 1,320 & 14,600 \\
\hline $3-14$ & Mississippi River below Belle Chasse, La. & 2,290 & 11,400 & 248,000 & $*$ & 1,660 & 1,890 & 4,610 \\
\hline
\end{tabular}


Table 52.- Discharge and corresponding transport data for dissolved trace elements in water samples collected from the Mississippi River and some of its tributaries during the May-June 1990 cruise

[ *, concentration less than the detection limit; All results are in kilograms per day except discharge, which is in cubic meters per second ]

\begin{tabular}{|c|c|c|c|c|c|c|c|c|}
\hline $\begin{array}{l}\text { Date } \\
1990\end{array}$ & Sampling site & Discharge & Aluminum & Arsenic & Barium & Beryllium & Boron & Cadmlum \\
\hline $6-07$ & Illinois River at Valley City, Ill. & 1,230 & 1,110 & 79 & 7,010 & $*$ & 10,200 & $*$ \\
\hline $6-11$ & Mississippi River below Grafton, III. & 5,040 & 2,520 & 446 & 33,500 & 12 & 14,500 & * \\
\hline $6-13$ & Mississippi River at Thebes, Ill. & 12,600 & 15,010 & 2,180 & 84,400 & 30 & 39,200 & * \\
\hline $6-14$ & Ohio River at Olmsted, Ill. & 9,550 & 9,150 & * & 26,000 & 39 & 24,400 & * \\
\hline $6-18$ & Mississippi River below Memphis, Tenn. & 20,800 & 23,200 & 1,690 & 110,500 & * & 73,500 & $*$ \\
\hline $6-20$ & Mississippi River below Arkansas City, Ark. & 25,500 & 25,000 & 2,200 & 131,000 & * & 76,100 & $*$ \\
\hline $6-22$ & Yazoo River below Steele Bayou, Miss. & 1,250 & 373 & 403 & 3,670 & * & 2,800 & * \\
\hline $6-23$ & Mississippi River below Vicksburg, Miss. & 27,300 & 11,200 & 3,490 & 134,000 & * & 91,200 & $*$ \\
\hline $6-25$ & Mississippi River near St. Francisville, La. & 23,200 & 26,600 & 3,800 & 116,200 & * & 63,200 & * \\
\hline 6-27 & Mississippi River below Belle Chasse, La. & 23,300 & 11,500 & 3,720 & 115,800 & * & 61,700 & * \\
\hline
\end{tabular}

Table 52.-- Discharge and corresponding transport data for dissolved trace elements in water samples collected from the Mississippi River and some of its tributaries during the May-June 1990 cruise - Continued

\begin{tabular}{|c|c|c|c|c|c|c|c|c|}
\hline Date & & & & & & & & \\
\hline 1990 & Sampling slte & Chromlum & Cobalt & Copper & Iron & Lead & Lithium & Manganese \\
\hline 6-07 & Illinois River at Valley City, Ill. & * & 75 & 219 & 829 & 12 & 455 & 850 \\
\hline $6-11$ & Mississippi River below Grafton, Ill. & * & 155 & 880 & * & * & 1,390 & 215 \\
\hline $6-13$ & Mississippi River at Thebes, 111. & * & 357 & 2,380 & 31,900 & 75 & 5,560 & 760 \\
\hline $6-14$ & Ohio River at Olmsted, Ill. & * & 368 & 1,370 & * & * & 1,520 & 391 \\
\hline $6-18$ & Mississippi River below Memphis, Tenn. & * & 505 & 3,400 & 15,800 & * & 6,780 & 1,350 \\
\hline $6-20$ & Mississippi River below Arkansas City, Ark. & 5,500 & 1,380 & 4,670 & $1,440,000$ & 1,360 & 8,320 & 74,700 \\
\hline $6-22$ & Yazoo River below Steele Bayou, Miss. & * & 22 & 211 & 1,210 & * & 185 & 7,290 \\
\hline $6-23$ & Mississippi River below Vicksburg, Miss. & * & 839 & 4,670 & * & * & 7,940 & 1,590 \\
\hline $6-25$ & Mississippi River near St. Francisville, La. & 460 & 749 & 4,100 & 189,000 & 237 & 6,440 & 18,500 \\
\hline $6-27$ & Mississippi River below Belle Chasse, La. & * & 556 & 3,660 & * & * & 6,620 & 1,500 \\
\hline
\end{tabular}

Table 52.-- Discharge and corresponding transport data for dissolved trace elements in water samples collected from the Mississippi River and some of its tributaries during the May-June 1990 cruise -- Continued

\begin{tabular}{|c|c|c|c|c|c|c|c|c|}
\hline $\begin{array}{l}\text { Date } \\
1990\end{array}$ & Sampling site & Molybdenum & Nickel & Strontium & Thallium & Uranium & Vanadium & Zinc \\
\hline 6-07 & Illinois River at Valley City, III. & 380 & 1,500 & 21,800 & $*$ & 187 & 196 & 260 \\
\hline $6-11$ & Mississippi River below Grafton, Ill. & 860 & 3,660 & 53,500 & * & 920 & 756 & 353 \\
\hline $6-13$ & Mississippi River at Thebes, Ill. & 1,900 & 8,060 & 171,000 & * & 1,780 & 2,300 & 1,580 \\
\hline $6-14$ & Ohio River at Olmsted, Ill. & 1,300 & 5,280 & 103,000 & * & 195 & 517 & 1,300 \\
\hline 6-18 & Mississippi River below Memphis, Tenn. & 3,100 & 11,800 & 269,000 & * & 1,490 & 2,660 & 1,480 \\
\hline 6-20 & Mississippi River below Arkansas City, Ark. & 2,200 & 15,400 & 316,000 & 112 & 1,790 & 5,950 & 6,100 \\
\hline $6-22$ & Yazoo River below Steele Bayou, Miss. & 78 & 334 & 6,130 & * & 12 & 122 & 130 \\
\hline $6-23$ & Mississippi River below Vicksburg, Miss. & 3,700 & 14,400 & 333,000 & 120 & 1,620 & 3,450 & 1,480 \\
\hline $6-25$ & Mississippi River near St. Francisville, La. & 3,100 & 13,600 & 289,000 & * & 1,750 & 3,370 & 2,920 \\
\hline $6-27$ & Mississippi River below Belle Chasse, La. & 3,600 & 13,300 & 293,000 & * & 1,360 & 3,280 & 1,850 \\
\hline
\end{tabular}


Table 53.- Discharge and corresponding transport data for dissolved major elements in water samples collected from the Mississippi River and some of its tributaries during the July-August 1987 cruise

[ --, not determined; All results are in kilograms per day except discharge, which is in cubic meters per second ]

\begin{tabular}{|c|c|c|c|c|c|c|c|}
\hline $\begin{array}{l}\text { Date } \\
1987\end{array}$ & Samplina site & Discharae & Calcium & Maanesium & Potassium & Sodium & Silica \\
\hline $7-17$ & Illinois River below Meredosia, IIl. & 312 & $1,700,000$ & 714,000 & 123,000 & $1,100,000$ & 12,500 \\
\hline $7-18$ & Mississippi River near Winfield, Mo. & 1,370 & $4,880,000$ & $2,430,000$ & 354,000 & $1,320,000$ & 610,000 \\
\hline $7-20$ & Missouri River at Hermann, Mo. & 2,640 & $10,200,000$ & $2,780,000$ & $1,330,000$ & $6,810,000$ & $3,470,000$ \\
\hline $7-21$ & Mississippi River at Hartford, $\mathrm{Ill}$. & 1,500 & $5,650,000$ & $2,640,000$ & 438,000 & $1,960,000$ & 997,000 \\
\hline $7-22$ & Mississippi River at St. Louis, Mo. & 3,940 & $15,700,000$ & $5,260,000$ & - & $8,560,000$ & $3,890,000$ \\
\hline 7.23 & Mississippi River at Chester, Ill. & 4,250 & $15,900,000$ & $5,520,000$ & $1,730,000$ & $8,510,000$ & $3,720,000$ \\
\hline 7.26 & $\begin{array}{l}\text { Ohio River below Smithland Locks and Dam, } \\
\text { Ill.-Ky. }\end{array}$ & 652 & - & - & - & - & - \\
\hline $7-27$ & Ohio River at Olmsted, III. & 2,070 & $6,520,000$ & $1,570,000$ & 492,000 & $2,300,000$ & 876,000 \\
\hline $7-28$ & Mississippi River below Hickman, Ky. & 6,270 & $24,100,000$ & $7,820,000$ & -- & $12,700,000$ & $4,630,000$ \\
\hline $7-30$ & Mississippi River at Helena, Ark. & 6,850 & $24,100,000$ & $7,530,000$ & $2,420,000$ & $11,100,000$ & $6,020,000$ \\
\hline 7.31 & White River at Mile 11.5, Ark. & 332 & 943,000 & 402,000 & 45,300 & 106,000 & 189,000 \\
\hline $8-01$ & Arkansas River at Mile 55.9, Ark. & 790 & $3,420,000$ & $1,010,000$ & 366,000 & $6,570,000$ & 225,000 \\
\hline $8-02$ & Mississippi River above Arkansas City, Ark. & 7,630 & $28,600,000$ & $8,970,000$ & $2,740,000$ & $18,500,000$ & $6,560,000$ \\
\hline $8-04$ & Mississippi River below Vicksburg, Miss. & 7,750 & $29,900,000$ & $9,140,000$ & $3,440,000$ & $18,200,000$ & $7,510,000$ \\
\hline $8-06$ & $\begin{array}{l}\text { Old River Outflow Channel near Knox } \\
\text { Landing, La. }\end{array}$ & 2,050 & $7,620,000$ & $2,370,000$ & 719,000 & $4,470,000$ & $1,550,000$ \\
\hline $8-07$ & Mississippi River near St. Francisville, La. & 6,190 & $23,500,000$ & $7,360,000$ & $2,170,000$ & $14,000,000$ & $5,370,000$ \\
\hline
\end{tabular}

Table 54.-- Discharge and corresponding transport data for dissolved major elements in water samples collected from the Mississippi River and some of its tributaries during the NovemberDecember 1987 cruise

[ All results are in kilograms per day except discharge, which is in cubic meters per second ]

\begin{tabular}{|c|c|c|c|c|c|c|c|}
\hline $\begin{array}{l}\text { Date } \\
1987\end{array}$ & Sampling site & Discharge & Calcium & Magnesium & Potassium & Sodium & Silica \\
\hline $11-29$ & Illinois River below Meredosia, III. & 262 & $1,440,000$ & 657,000 & 103,000 & 996,000 & 75,300 \\
\hline $11-30$ & Mississippi River near Winfield, Mo. & 2,040 & $7,290,000$ & $3,750,000$ & 478,000 & $2,170,000$ & 199,000 \\
\hline $12-02$ & Missouri River at St. Charles, Mo. & 2,810 & $13,500,000$ & $4,610,000$ & $1,140,000$ & $10,500,000$ & $2,770,000$ \\
\hline $12-03$ & Mississippi River at St. Louis, Mo. & 5,440 & $22,800,000$ & $9,290,000$ & $2,170,000$ & $12,600,000$ & $3,680,000$ \\
\hline $12-05$ & Mississippi River at Thebes, III. & 5,190 & $21,000,000$ & $8,630,000$ & $1,760,000$ & $11,800,000$ & $3,900,000$ \\
\hline $12-06$ & Ohio River at Olmsted, Ill. & 4,200 & $13,700,000$ & $4,040,000$ & $1,190,000$ & $8,590,000$ & $2,160,000$ \\
\hline 12-07 & Mississippi River below Hickman, Ky. & 8,820 & $33,400,000$ & $11,900,000$ & $2,710,000$ & $19,100,000$ & $5,030,000$ \\
\hline $12-08$ & Mississippi River at Fulton, Tenn. & 9,470 & $35,900,000$ & $12,900,000$ & $2,900,000$ & $21,300,000$ & $5,650,000$ \\
\hline $12-11$ & Mississippi River at Helena, Ark. & 8,770 & $32,100,000$ & $11,400,000$ & $2,630,000$ & $18,700,000$ & $4,930,000$ \\
\hline $12-12$ & White River at Mile 11.5, Ark. & 519 & $1,020,000$ & 396,000 & 109,000 & 165,000 & 419,000 \\
\hline $12-13$ & Mississippi River above Arkansas City, Ark. & 9,920 & $34,700,000$ & $13,000,000$ & $2,970,000$ & $23,700,000$ & $4,530,000$ \\
\hline $12-14$ & Yazoo River at Mile 10, Miss. & 177 & 123,000 & 42,500 & 50,800 & 87,700 & 159,000 \\
\hline $12-15$ & Mississippi River below Vicksburg, Miss. & 10,410 & $34,300,000$ & $12,400,000$ & $3,150,000$ & $22,900,000$ & $5,300,000$ \\
\hline $12-17$ & $\begin{array}{l}\text { Old River Outflow Channel near Knox } \\
\text { Landing, La. }\end{array}$ & 1,830 & $6,140,000$ & $2,220,000$ & 591,000 & $4,200,000$ & $1,050,000$ \\
\hline 12-18 & Mississippi River near St. Francisville, La. & 8,180 & $28,000,000$ & $10,200,000$ & $2,470,000$ & $19,200,000$ & $5,160,000$ \\
\hline $12-20$ & Mississippi River below Belle Chasse, La. & 9,560 & $30,300,000$ & $11,300,000$ & $3,020,000$ & $27,700,000$ & $5,060,000$ \\
\hline
\end{tabular}


Table 55.- Discharge and corresponding transport data for dissolved major elements in water samples collected from the Mississippi River and some of its tributaries during the May-June 1988 cruise

[ All results are in kilograms per day except discharge, which is in cubic meters per second ]

\begin{tabular}{|c|c|c|c|c|c|c|c|}
\hline $\begin{array}{l}\text { Date } \\
1988\end{array}$ & Sampling site & Discharge & Calcium & Magneslum & Potasslum & Sodium & Sillca \\
\hline $5-16$ & Illinois River below Meredosia, Ill. & 332 & $1,750,000$ & 866,000 & 116,000 & 978,000 & 21,900 \\
\hline $5-17$ & Mississippi River near Winfield, Mo. & 1,740 & $5,490,000$ & $3,250,000$ & 499,000 & $1,720,000$ & 47,200 \\
\hline $5-19$ & Missouri River at Hermann, Mo. & 1,480 & $8,040,000$ & $2,740,000$ & 941,000 & $6,920,000$ & $1,240,000$ \\
\hline $5-20$ & Mississippi River at St. Louis, Mo. & 3,350 & $15,000,000$ & $6,630,000$ & $1,620,000$ & $9,200,000$ & $1,180,000$ \\
\hline $5-22$ & Mississippi River at Thebes, Ill. & 3,590 & $16,700,000$ & $7,230,000$ & $1,430,000$ & $10,300,000$ & $1,210,000$ \\
\hline $5-23$ & Ohio River at Olmsted, III. & 3,230 & $10,200,000$ & $3,400,000$ & 834,000 & $4,790,000$ & 93,900 \\
\hline $5-24$ & Mississippi River below Hickman, Ky. & 6,790 & $26,900,000$ & $10,600,000$ & $2,280,000$ & $15,000,000$ & $1,340,000$ \\
\hline $5-26$ & Mississippi River at Fulton, Tenn. & 7,170 & $27,100,000$ & $10,500,000$ & $2,420,000$ & $14,700,000$ & $1,250,000$ \\
\hline 5.28 & Mississippi River at Helena, Ark. & 7,050 & $25,800,000$ & $9,930,000$ & $2,430,000$ & $13,600,000$ & $1,500,000$ \\
\hline 5.29 & White River at Mile 11.5, Ark. & 438 & $1,240,000$ & 455,000 & 147,000 & 134,000 & 148,000 \\
\hline $5-29$ & Arkansas River at Mile 1.0, Ark. & 8,160 & $29,700,000$ & $11,300,000$ & $2,710,000$ & $15,200,000$ & $1,390,000$ \\
\hline $5-30$ & Mississippi River above Arkansas City, Ark. & 73 & 156,000 & 50,000 & 21,400 & 78,800 & 64,800 \\
\hline $6-02$ & Mississippi River below Vicksburg, Miss. & 7,950 & $30,800,000$ & $11,600,000$ & $2,620,000$ & $15,900,000$ & $1,190,000$ \\
\hline 6-04 & $\begin{array}{l}\text { Old River Outflow Channel near Knox } \\
\text { Landing, La. }\end{array}$ & 2,150 & $8,330,000$ & $3,080,000$ & 711,000 & $4,260,000$ & 353,000 \\
\hline 6-05 & Mississippi River near St. Francisville, La. & 5,700 & $22,900,000$ & $8,400,000$ & $1,890,000$ & $11,600,000$ & 959,000 \\
\hline 6.07 & Mississippi River below Belle Chasse, La. & 5,570 & $22,800,000$ & $8,370,000$ & $1,970,000$ & $15,400,000$ & $1,020,000$ \\
\hline
\end{tabular}

Table 56.-- Discharge and corresponding transport data for dissolved major elements in water samples collected from the Mississippi River and some of its tributaries during the March-April 1989 cruise

[ All results are in kilograms per day except discharge, which is in cubic meters per second ]

\begin{tabular}{|c|c|c|c|c|c|c|c|}
\hline $\begin{array}{l}\text { Date } \\
1989\end{array}$ & Sampling slte & Discharge & Calcium & Magnesium & Potasslum & Sodium & sillca \\
\hline 3-09 & Illinois River at Hardin, Ill. & 410 & $2,820,000$ & $1,110,000$ & 149,000 & $2,600,000$ & 186,000 \\
\hline $3-10$ & Mississippi River near Winfield, Mo. & 850 & $3,700,000$ & $1,550,000$ & 259,000 & $1,330,000$ & 341,000 \\
\hline $3-12$ & Missouri River at Hermann, Mo. & 1,480 & $5,700,000$ & $2,090,000$ & 471,000 & $4,160,000$ & $1,180,000$ \\
\hline $3-13$ & Mississippi River at St. Louis, Mo. & 3,940 & $17,600,000$ & $6,750,000$ & $1,250,000$ & $12,200,000$ & $1,930,000$ \\
\hline $3-15$ & Mississippi River at Thebes, Ill. & 4,890 & $20,300,000$ & $7,850,000$ & $1,550,000$ & $13,300,000$ & $2,420,000$ \\
\hline 3-16 & Ohio River at Olmsted, III. & 20,400 & $48,500,000$ & $11,000,000$ & $2,960,000$ & $13,000,000$ & $9,470,000$ \\
\hline $3-17$ & Mississippi River below Hickman, Ky. & 24,700 & $67,100,000$ & $18,500,000$ & $4,860,000$ & $25,800,000$ & $11,200,000$ \\
\hline $3-19$ & Mississippi River below Fulton, Tenn. & 24,800 & $66,100,000$ & $18,300,000$ & $4,440,000$ & $24,800,000$ & $11,100,000$ \\
\hline $3-21$ & Mississippi River at Helena, Ark. & 25,900 & $70,600,000$ & $19,400,000$ & $4,630,000$ & $26,500,000$ & $12,700,000$ \\
\hline $3-22$ & White River at Mile 11.5, Ark. & 1,500 & $2,330,000$ & 870,000 & 194,000 & 232,000 & 573,000 \\
\hline $3-23$ & Arkansas River at Pendleton, Ark. & 1,900 & $4,000,000$ & $1,030,000$ & 364,000 & $5,120,000$ & 621,000 \\
\hline 3.24 & Mississippi River above Arkansas City, Ark. & 26,800 & $74,400,000$ & $21,500,000$ & $5,310,000$ & $31,500,000$ & $12,700,000$ \\
\hline $3-26$ & Yazoo River below Steele Bayou, Miss. & 1,500 & 921,000 & 287,000 & 306,000 & 413,000 & 671,000 \\
\hline 3-27 & Mississippi River below Vicksburg, Miss. & 26,600 & $72,300,000$ & $20,600,000$ & $5,390,000$ & $31,500,000$ & $12,600,000$ \\
\hline 3-29 & $\begin{array}{l}\text { Old River Outflow Channel near Knox } \\
\text { Landing, La. }\end{array}$ & 6,160 & $16,800,000$ & $4,700,000$ & $1,260,000$ & $7,240,000$ & $2,910,000$ \\
\hline 3-30 & Mississippi River near St. Francisville, La. & 23,100 & $61,900,000$ & $17,500,000$ & $4,490,000$ & $27,000,000$ & $10,800,000$ \\
\hline 4-01 & Mississippi River below Belle Chasse, La. & 22,500 & $60,400,000$ & $16,700,000$ & $4,680,000$ & $29,400,000$ & $10,600,000$ \\
\hline
\end{tabular}


Table 57. - Discharge and corresponding transport data for dissolved major elements in water samples collected from the Mississippi River and some of its tributaries during the June 1989 cruise

[ All results are in kilograms per day except discharge, which is in cubic meters per second ]

\begin{tabular}{|c|c|c|c|c|c|c|c|}
\hline $\begin{array}{l}\text { Date } \\
1989\end{array}$ & Sampling site & Discharge & Calcium & Magnesium & Potassium & Sodium & Sllica \\
\hline $6-04$ & Illinois River at Hardin, Ill. & 780 & $4,700,000$ & $2,130,000$ & 276,000 & $2,770,000$ & 223,000 \\
\hline $6-05$ & Mississippi River near Winfield, Mo. & 2,320 & $7,670,000$ & $3,880,000$ & 661,000 & $2,550,000$ & 263,000 \\
\hline $6-07$ & Missouri River at Hermann, Mo. & 1,760 & $8,060,000$ & $3,300,000$ & 776,000 & $7,830,000$ & $1,190,000$ \\
\hline $6-08$ & Mississippi River at St. Louis, Mo. & 4,760 & $20,100,000$ & $8,340,000$ & $1,690,000$ & $13,700,000$ & $1,870,000$ \\
\hline $6-10$ & Mississippi River at Thebes, Ill. & 5,230 & $21,800,000$ & $9,160,000$ & $1,850,000$ & $14,400,000$ & $1,950,000$ \\
\hline $6-11$ & Ohio River at Olmsted, 111. & 8,760 & $30,300,000$ & $8,510,000$ & $1,670,000$ & $7,170,000$ & $4,120,000$ \\
\hline $6-12$ & Mississippi River below Hickman, Ky. & 14,100 & $48,600,000$ & $17,500,000$ & $3,410,000$ & $20,100,000$ & $5,790,000$ \\
\hline $6-14$ & Mississippi River at Fulton, Tenn. & 15,300 & $53,400,000$ & $18,600,000$ & $3,830,000$ & $22,100,000$ & $6,440,000$ \\
\hline $6-17$ & Mississippi River at Helena, Ark. & 16,900 & $56,900,000$ & $20,300,000$ & $4,240,000$ & $21,900,000$ & $8,220,000$ \\
\hline 6-18 & White River at Mile 11.5, Ark. & 770 & $2,250,000$ & $1,120,000$ & 134,000 & 258,000 & 595,000 \\
\hline $6-19$ & Arkansas River at Pendleton, Ark. & 3,600 & $10,600,000$ & $2,910,000$ & 931,000 & $25,300,000$ & $1,830,000$ \\
\hline $6-20$ & Mississippi River above Arkansas City, Ark. & 23,300 & $68,800,000$ & $23,800,000$ & $5,240,000$ & $44,300,000$ & $12,100,000$ \\
\hline $6-22$ & Yazoo River below Steele Bayou, Miss. & 1,070 & 519,000 & 168,000 & 240,000 & 243,000 & 653,000 \\
\hline $6-23$ & Mississippi River below Vicksburg, Miss. & 24,800 & $72,000,000$ & $22,300,000$ & $5,790,000$ & $42,800,000$ & $12,400,000$ \\
\hline $6-25$ & $\begin{array}{l}\text { Old River Outflow Channel near Knox } \\
\text { Landing, La. }\end{array}$ & 4,890 & $13,500,000$ & $3,990,000$ & $1,050,000$ & $8,010,000$ & $2,360,000$ \\
\hline $6-26$ & Mississippi River near St. Francisville, La. & 19,000 & $54,200,000$ & $15,800,000$ & $4,280,000$ & $31,200,000$ & $9,300,000$ \\
\hline $6-28$ & Mississippi River below Belle Chasse, La. & 20,100 & $50,600,000$ & $15,200,000$ & $4,330,000$ & $34,500,000$ & $8,950,000$ \\
\hline
\end{tabular}

Table 58. - Discharge and corresponding transport data for dissolved major elements in water samples collected from the Mississippi River and some of its tributaries during the FebruaryMarch 1990 cruise

[ All results are in kilograms per day except discharge, which is in cubic meters per second ]

\begin{tabular}{|c|c|c|c|c|c|c|c|}
\hline $\begin{array}{l}\text { Date } \\
1990\end{array}$ & Sampiing site & Discharge & Calcium & Magnesium & Potassium & Sodium & Silica \\
\hline $2-23$ & Cumberland River near Smithland, Ky. & 2,170 & $5,700,000$ & 775,000 & 281,000 & 561,000 & 946,000 \\
\hline $2-24$ & Tennessee River near Calvert City, Ky. & 6,570 & $9,950,000$ & $1,680,000$ & 738,000 & $1,980,000$ & $3,050,000$ \\
\hline $2-25$ & Mississippi River near Cache, Ill. & 4,240 & $15,900,000$ & $7,660,000$ & $1,770,000$ & $9,140,000$ & $2,550,000$ \\
\hline 2-28 & Wabash River near New Haven, Ill. & 2,340 & $9,110,000$ & $3,250,000$ & 647,000 & $1,590,000$ & $1,440,000$ \\
\hline 3-01 & Ohio River at Uniontown, Ky. & 6,620 & $19,000,000$ & $5,150,000$ & $1,370,000$ & $5,280,000$ & $3,840,000$ \\
\hline $3-03$ & Ohio River at Olmsted, Ill. & 16,100 & $42,700,000$ & $11,100,000$ & $2,920,000$ & $9,750,000$ & $9,100,000$ \\
\hline 3-04 & Mississippi River below Hickman, Ky. & 21,000 & $60,100,000$ & $17,300,000$ & $4,900,000$ & $18,900,000$ & $11,800,000$ \\
\hline $3-05$ & Mississippi River below Fulton, Tenn. & 22,800 & $62,300,000$ & $20,200,000$ & $5,120,000$ & $19,400,000$ & $13,200,000$ \\
\hline 3-07 & Mississippi River at Helena, Ark. & 23,330 & $62,300,000$ & $20,400,000$ & $5,650,000$ & $19,600,000$ & $13,200,000$ \\
\hline 3-08 & Mississippi River above Arkansas City, Ark. & 33,200 & $88,600,000$ & $30,000,000$ & $6,600,000$ & $34,400,000$ & $19,000,000$ \\
\hline $3-10$ & Mississippi River below Vicksburg, Miss. & 34,100 & $72,400,000$ & $23,000,000$ & $6,780,000$ & $29,500,000$ & $16,400,000$ \\
\hline $3-12$ & Mississippi River near St. Francisvilie, La. & 26,300 & $61,400,000$ & $19,100,000$ & $5,460,000$ & $24,600,000$ & $13,100,000$ \\
\hline 3-14 & Mississippi River below Belle Chasse, La. & 26,700 & $61,300,000$ & $19,000,000$ & $5,770,000$ & $27,900,000$ & $13,500,000$ \\
\hline
\end{tabular}


Table 59.- Discharge and corresponding transport data for dissolved major elements in water samples collected from the Mississippi River and some of its tributaries during the May-June 1990 cruise

[ All results are in kilograms per day except discharge, which is in cubic meters per second ]

\begin{tabular}{|c|c|c|c|c|c|c|c|}
\hline $\begin{array}{l}\text { Date } \\
1990\end{array}$ & Sampling site & Discharge & Calcium & Magnesium & Potassium & Sodium & Silica \\
\hline $6-06$ & Illinois River at Valley City, Ill. & 1,230 & $8,100,000$ & $3,460,000$ & 329,000 & $2,730,000$ & 677,000 \\
\hline $6-08$ & Mississippi River below Grafton, Ill. & 5,040 & $21,700,000$ & $7,880,000$ & $1,610,000$ & $4,880,000$ & $2,860,000$ \\
\hline $6-13$ & Mississippi River at Thebes, Ill. & 12,600 & $43,100,000$ & $13,700,000$ & $3,920,000$ & $14,000,000$ & $8,130,000$ \\
\hline $6-14$ & Ohio River at Olmsted, Ill. & 9,550 & $28,600,000$ & $6,860,000$ & $1,980,000$ & $5,900,000$ & $4,500,000$ \\
\hline $6-17$ & Mississippi River below Memphis, Tenn. & 20,800 & $68,400,000$ & $19,400,000$ & $6,470,000$ & $19,700,000$ & $12,000,000$ \\
\hline $6-20$ & Mississippi River below Arkansas City, Ark. & 25,500 & $81,000,000$ & $23,200,000$ & $7,040,000$ & $23,900,000$ & $20,200,000$ \\
\hline $6-22$ & Yazoo River below Steele Bayou, Miss. & 1,250 & $1,180,000$ & 364,000 & 346,000 & 526,000 & 698,000 \\
\hline $6-23$ & Mississippi River below Vicksburg, Miss. & 27,300 & $84,300,000$ & $23,500,000$ & $8,020,000$ & $25,300,000$ & $15,800,000$ \\
\hline $6-25$ & Mississippi River near St. Francisville, La. & 23,200 & $71,000,000$ & $19,800,000$ & $6,610,000$ & $21,400,000$ & $14,400,000$ \\
\hline $6-27$ & Mississippi River below Belle Chasse, La. & 23,300 & $71,400,000$ & $21,000,000$ & $6,640,000$ & $23,500,000$ & $14,100,000$ \\
\hline
\end{tabular}

Table 60.- Discharge and corresponding transport data for dissolved major anions in water samples collected from the Mississippi River and some of its tributaries during the July-August 1987 cruise

[ --, not determined; All results are in kilograms per day except discharge, which is in cubic meters per second ]

\begin{tabular}{|c|c|c|c|c|c|c|}
\hline $\begin{array}{l}\text { Date } \\
1987\end{array}$ & Sampling site & Discharge & Chloride & Sulfate & $\begin{array}{c}\text { Nitrate as } \\
\text { nitrogen }\end{array}$ & Bicarbonate \\
\hline $7-17$ & Illinois River below Meredosia, Ill. & 312 & - & - & - & - \\
\hline $7-18$ & Mississippi River near Winfield, Mo. & 1,370 & $1,800,000$ & $4,200,000$ & 200,000 & $21,400,000$ \\
\hline $7-20$ & Missouri River at Hermann, Mo. & 2,640 & $4,000,000$ & $17,900,000$ & 420,000 & $32,000,000$ \\
\hline $7-21$ & Mississippi River at Hartford, Ill. & 1,500 & $2,600,000$ & $5,200,000$ & 250,000 & $22,700,000$ \\
\hline $7-22$ & Mississippi River at St. Louis, Mo. & 3,940 & $6,700,000$ & $22,600,000$ & 550,000 & $52,500,000$ \\
\hline $7-23$ & Mississippi River at Chester, Ill. & 4,250 & $6,900,000$ & $23,400,000$ & 640,000 & $56,700,000$ \\
\hline $7-26$ & $\begin{array}{l}\text { Ohio River below Smithland Locks and Dam, } \\
\text { Ill.-Ky. }\end{array}$ & 652 & - & - & - & - \\
\hline $7-27$ & Ohio River at Olmsted, Ill. & 2,070 & $2,500,000$ & $8,100,000$ & 640,000 & $17,000,000$ \\
\hline $7-28$ & Mississippi River below Hickman, Ky. & 6,270 & $9,800,000$ & $37,100,000$ & 860,000 & $79,700,000$ \\
\hline $7-30$ & Mississippi River at Helena, Ark. & 6,850 & $9,100,000$ & $30,300,000$ & 850,000 & $80,500,000$ \\
\hline $7-31$ & White River at Mile 11.5, Ark. & 332 & 170,000 & 200,000 & 8,000 & $4,700,000$ \\
\hline $8-01$ & Arkansas River at Mile 55.9, Ark. & 790 & $9,000,000$ & $6,200,000$ & 30,000 & $9,900,000$ \\
\hline $8-02$ & Mississippi River above Arkansas City, Ark. & 7,630 & $19,600,000$ & $40,300,000$ & - & $1,100,000$ \\
\hline $8-04$ & Mississippi River below Vicksburg, Miss. & 7,750 & $18,500,000$ & $38,900,000$ & 980,000 & $99,400,000$ \\
\hline $8-06$ & $\begin{array}{l}\text { Old River Outflow Channel near Knox } \\
\text { Landing, La. }\end{array}$ & 2,050 & - & - & -- & - \\
\hline 8-07 & Mississippi River near St. Francisville, La. & 6,190 & $14,200,000$ & $29,700,000$ & 770,000 & $80,800,000$ \\
\hline
\end{tabular}


Table 61.- Discharge and corresponding transport data for dissolved major anions in water samples collected from the Mississippi River and some of its tributaries during the NovemberDecember 1987 cruise

[ -, not determined; All results are in kilograms per day except discharge, which is in cubic meters per second ]

\begin{tabular}{|c|c|c|c|c|c|c|}
\hline \multirow{2}{*}{$\begin{array}{l}\text { Date } \\
1987\end{array}$} & \multirow[b]{2}{*}{ Sampling site } & \multirow[b]{2}{*}{ Discharge } & \multirow[b]{2}{*}{ Chloride } & \multicolumn{3}{|c|}{ Nitrate as } \\
\hline & & & & Sulfate & nltrogen & Blcarbonate \\
\hline $11-29$ & Illinois River below Meredosia, Ill. & 262 & - & - & 73,000 & - \\
\hline $11-30$ & Mississippi River near Winfield, Mo. & 2,040 & $3,200,000$ & $5,100,000$ & 270,000 & $34,600,000$ \\
\hline $12-02$ & Missouri River at St. Charles, Mo. & 2,810 & $3,800,000$ & $30,600,000$ & 280,000 & -. \\
\hline $12-03$ & Mississippi River at St. Louis, Mo. & 5,440 & $8,700,000$ & $34,600,000$ & 670,000 & $91,200,000$ \\
\hline $12-05$ & Mississippi River at Thebes, Ill. & 5,190 & $8,800,000$ & $35,100,000$ & 670,000 & $86,600,000$ \\
\hline $12-06$ & Ohio River at Olmsted, Ill. & 4,200 & $9,300,000$ & $25,500,000$ & 460,000 & - \\
\hline 12-07 & Mississippi River below Hickman, Ky. & 8,820 & $16,800,000$ & $54,200,000$ & $1,090,000$ & $112,000,000$ \\
\hline $12-08$ & Mississippi River at Fulton, Tenn. & 9,470 & $17,000,000$ & $57,300,000$ & $1,040,000$ & $120,000,000$ \\
\hline $12-11$ & Mississippi River at Helena, Ark. & 8,770 & $16,000,000$ & $51,700,000$ & $1,040,000$ & $118,000,000$ \\
\hline $12-12$ & White River at Mile 11.5, Ark. & 519 & 300,000 & 370,000 & 18,000 & - \\
\hline 12-13 & Mississippi River above Arkansas City, Ark. & 9,920 & $22,800,000$ & $52,400,000$ & $1,070,000$ & - \\
\hline $12-14$ & Yazoo River at Mile 10, Miss. & 177 & $1,000,000$ & 100,000 & 7,000 & - \\
\hline $12-15$ & Mississippi River below Vicksburg, Miss. & 10,410 & $23,700,000$ & $54,500,000$ & $1,090,000$ & $123,000,000$ \\
\hline $12-17$ & $\begin{array}{l}\text { Old River Outflow Channel near Knox } \\
\text { Landing, La. }\end{array}$ & 1,830 & - & - & - & - \\
\hline $12-18$ & Mississippi River near St. Francisville, La. & 8,180 & $19,000,000$ & $44,300,000$ & 920,000 & $9,500,000$ \\
\hline $12-20$ & Mississippi River below Belle Chasse, La. & 9,560 & $32,800,000$ & $50,300,000$ & $1,040,000$ & $110,000,000$ \\
\hline
\end{tabular}

Table 62.-- Discharge and corresponding transport data for dissolved major anions in water samples collected from the Mississippi River and some of its tributaries during the May-June 1988 cruise

[ -, not determined; All results are in kilograms per day except discharge, which is in cubic meters per second ]

\begin{tabular}{|c|c|c|c|c|c|c|}
\hline $\begin{array}{l}\text { Date } \\
1988\end{array}$ & Sampling site & Discharge & Chloride & Sulfate & $\begin{array}{c}\text { Nitrate as } \\
\text { nitrogen }\end{array}$ & Bicarbonate \\
\hline $5-16$ & Illinois River below Meredosia, Ill. & 332 &.- & - & 97,000 & $6,800,000$ \\
\hline $5-17$ & Mississippi River near Winfield, Mo. & 1,740 & $2,600,000$ & $5,500,000$ & 190,000 & $25,100,000$ \\
\hline 5-19 & Missouri River at Hermann, Mo. & 1,480 & $2,500,000$ & $1,900,000$ & 120,000 & $21,900,000$ \\
\hline $5-20$ & Mississippi River at St. Louis, Mo. & 3,350 & $6,300,000$ & $25,600,000$ & 370,000 & $52,400,000$ \\
\hline $5-22$ & Mississippi River at Thebes, Ill. & 3,590 & $6,800,000$ & $28,200,000$ & 430,000 & $54,200,000$ \\
\hline 5.23 & Ohio River at Olmsted, Ill. & 3,230 & $4,300,000$ & $13,800,000$ & 190,000 & $32,100,000$ \\
\hline $5-24$ & Mississippi River below Hickman, Ky. & 6,790 & $11,800,000$ & $44,300,000$ & 710,000 & $86,500,000$ \\
\hline $5-26$ & Mississippi River at Fulton, Tenn. & 7,170 & $11,900,000$ & $44,600,000$ & 600,000 & $83,700,000$ \\
\hline $5-28$ & Mississippi River at Helena, Ark. & 7,050 & $10,900,000$ & $38,600,000$ & 590,000 & $80,700,000$ \\
\hline $5-29$ & White River at Mile 11.5, Ark. & 438 & 250,000 & 280,000 & - & $6,000,000$ \\
\hline $5-29$ & Mississippi River above Arkansas City, Ark. & 8,160 & $13,600,000$ & $46,800,000$ & 700,000 & $106,000,000$ \\
\hline 5.30 & Yazoo River at Mile 10, Miss & 73 & 450,000 & 590,000 & - & 780,000 \\
\hline $6-02$ & Mississippi River below Vicksburg, Miss. & 7,950 & $13,400,000$ & $44,200,000$ & 610,000 & $81,000,000$ \\
\hline $6-04$ & $\begin{array}{l}\text { Old River Outflow Channel near Knox } \\
\text { Landing, La. }\end{array}$ & 2,150 & -- & - & - & - \\
\hline $6-05$ & Mississippi River near St. Francisville, La. & 5,700 & $6,070,000$ & $16,900,000$ & 300,000 & $60,300,000$ \\
\hline $6-07$ & Mississippi River below Belle Chasse, La. & 5,570 & $15,100,000$ & $30,300,000$ & 490,000 & $67,800,000$ \\
\hline
\end{tabular}


Table 63.- Discharge and corresponding transport data for dissolved major anions in water samples collected from the Mississippi River and some of its tributaries during the March-April 1989 cruise

[ --, not determined; All results are in kilograms per day except discharge, which is in cubic meters per second ]

\begin{tabular}{|c|c|c|c|c|c|}
\hline $\begin{array}{l}\text { Date } \\
1989\end{array}$ & Sampling site & Discharge & Chioride & Suifate & Bicarbonate \\
\hline 3-09 & Illinois River at Hardin, III. & 410 & $3,800,000$ & $3,700,000$ & $3,100,000$ \\
\hline $3-10$ & Mississippi River near Winfield, Mo. & 850 & $1,800,000$ & $2,200,000$ & - \\
\hline $3-12$ & Missouri River at Hermann, Mo. & 1,480 & $2,000,000$ & $9,600,000$ & $17,500,000$ \\
\hline 3-13 & Mississippi River at St. Louis, Mo. & 3,940 & $14,000,000$ & $20,400,000$ & $48,100,000$ \\
\hline $3-15$ & Mississippi River at Thebes, Ill. & 4,890 & $14,200,000$ & $25,200,000$ & $57,600,000$ \\
\hline $3-16$ & Ohio River at Olmsted, Ill. & 20,400 & $17,000,000$ & $65,200,000$ & $113,000,000$ \\
\hline $3-17$ & Mississippi River below Hickman, Ky. & 24,700 & $30,100,000$ & $86,100,000$ & $180,000,000$ \\
\hline 3-19 & Mississippi River below Fulton, Tenn. & 24,800 & $30,500,000$ & $87,400,000$ & $185,000,000$ \\
\hline 3-21 & Mississippi River at Helena, Ark. & 25,900 & $32,100,000$ & $88,500,000$ & $197,000,000$ \\
\hline $3-22$ & White River at Mile 11.5, Ark. & 1,500 & 460,000 & 710,000 & $10,500,000$ \\
\hline 3-23 & Arkansas River at Pendleton, Ark. & 1,900 & $6,900,000$ & $5,100,000$ & $11,900,000$ \\
\hline $3-24$ & Mississippi River above Arkansas City, Ark. & 26,800 & $37,000,000$ & $87,400,000$ & $226,000,000$ \\
\hline 3-26 & Yazoo River below Steele Bayou, Miss. & 1,500 & 270,000 & 660,000 & $3,600,000$ \\
\hline 3-27 & Mississippi River below Vicksburg, Miss. & 26,600 & $36,400,000$ & $87,500,000$ & $225,000,000$ \\
\hline 3-29 & $\begin{array}{l}\text { Old River Outflow Channel near Knox } \\
\text { Landing, La. }\end{array}$ & 6,160 & - & - & $51,100,000$ \\
\hline $3-30$ & Mississippi River near St. Francisville, La. & 23,100 & $30,200,000$ & $72,700,000$ & $191,000,000$ \\
\hline 4-01 & Mississippi River below Belle Chasse, La. & 22,500 & $32,900,000$ & $68,300,000$ & $182,000,000$ \\
\hline
\end{tabular}

Table 64.-- Discharge and corresponding transport data for dissolved major anions in water samples collected from the Mississippi River and some of its tributaries during the June 1989 cruise

[ --, not determined; All results are in kilograms per day except discharge, which is in cubic meters per second ]

\begin{tabular}{|c|c|c|c|c|c|}
\hline $\begin{array}{l}\text { Date } \\
1989\end{array}$ & Sampling site & Discharge & Chioride & Suifate & Bicarbonate \\
\hline $6-04$ & Illinois River at Hardin, Ill. & 780 & -- & - & - \\
\hline $6-05$ & Mississippi River near Winfield, Mo. & 2,320 & - & -- & - \\
\hline $6-07$ & Missouri River at Hermann, Mo. & 1,760 & - & - & $11,700,000$ \\
\hline $6-08$ & Mississippi River at St. Louis, Mo. & 4,760 & $10,300,000$ & $33,900,000$ & $60,700,000$ \\
\hline $6-10$ & Mississippi River at Thebes, Ill. & 5,230 & $11,600,000$ & $36,500,000$ & $73,800,000$ \\
\hline $6-11$ & Ohio River at Olmsted, Ill. & 8,760 & $9,400,000$ & $35,200,000$ & $62,400,000$ \\
\hline $6-12$ & Mississippi River below Hickman, Ky. & 14,100 & $20,100,000$ & $66,400,000$ & $133,000,000$ \\
\hline $6-14$ & Mississippi River at Fulton, Tenn. & 15,300 & $21,000,000$ & $70,300,000$ & $164,000,000$ \\
\hline $6-17$ & Mississippi River at Helena, Ark. & 16,900 & $21,400,000$ & $68,300,000$ & $173,000,000$ \\
\hline $6-18$ & White River at Mile 11.5, Ark. & 770 & 300,000 & 380,000 & - \\
\hline 6-19 & Arkansas River at Pendleton, Ark. & 3,600 & $32,000,000$ & $14,100,000$ & $27,300,000$ \\
\hline $6-20$ & Mississippi River above Arkansas City, Ark. & 23,300 & $58,400,000$ & $81,200,000$ & $216,000,000$ \\
\hline $6-22$ & Yazoo River below Steele Bayou, Miss. & 1,070 & 370,000 & 410,000 & $1,900,000$ \\
\hline $6-23$ & Mississippi River below Vicksburg, Miss. & 24,800 & $52,800,000$ & $77,500,000$ & $218,000,000$ \\
\hline 6-25 & $\begin{array}{l}\text { Old River Outflow Channel near Knox } \\
\text { Landing, La. }\end{array}$ & 4,890 & - & - & $39,500,000$ \\
\hline $6-26$ & Mississippi River near St. Francisville, La. & 19,000 & $38,500,000$ & $58,900,000$ & $164,000,000$ \\
\hline $6-28$ & Mississippi River below Belle Chasse, La. & 20,100 & $51,100,000$ & $64,900,000$ & $178,000,000$ \\
\hline
\end{tabular}


Table 65.- Discharge and corresponding transport data for dissolved major anions in water samples collected from the Mississippi River and some of its tributaries during the FebruaryMarch 1990 cruise

[ --, not determined; All results are in kilograms per day except discharge, which is in cubic meters per second ]

\begin{tabular}{|c|c|c|c|c|c|c|}
\hline \multicolumn{4}{|l|}{ Date } & \multicolumn{3}{|c|}{ Nitrate as } \\
\hline 1990 & Sampling site & Discharge & Chloride & Sulfate & nitrogen & Bicarbonate \\
\hline $2-23$ & Cumberland River near Smithland, Ky. & 2,170 & - & - & 3,000 & $17,800,000$ \\
\hline $2-24$ & Tennessee River near Calvert City, Ky. & 6,570 & - & - & 5,000 & $31,500,000$ \\
\hline $2-25$ & Mississippi River near Cache, Ill. & 4,240 & $12,400,000$ & $24,700,000$ & $1,100,000$ & $68,300,000$ \\
\hline $2-28$ & Wabash River near New Haven, Ill. & 2,340 & -- & - & 2,000 & $29,900,000$ \\
\hline $3-01$ & Ohio River at Uniontown, Ky. & 6,620 & $7,400,000$ & $27,000,000$ & $1,100,000$ & $36,700,000$ \\
\hline $3-03$ & Ohio River at Olmsted, III. & 16,100 & $14,300,000$ & $50,000,000$ & $2,600,000$ & $118,000,000$ \\
\hline 3-04 & Mississippi River beiow Hickman, Ky. & 21,000 & - & - & -. & - \\
\hline 3-05 & Mississippi River below Fulton, Tenn. & 22,800 & $25,200,000$ & $73,700,000$ & $2,000,000$ & $117,000,000$ \\
\hline 3-07 & Mississippi River at Helena, Ark. & 23,300 & $26,800,000$ & $75,800,000$ & $2,800,000$ & $121,000,000$ \\
\hline $3-08$ & Mississippi River above Arkansas City, Ark. & 33,200 & $54,200,000$ & $94,100,000$ & $3,400,000$ & $160,000,000$ \\
\hline $3-10$ & Mississippi River below Vicksburg, Miss. & 34,100 & $47,200,000$ & $94,000,000$ & $3,400,000$ & $238,000,000$ \\
\hline $3-12$ & Mississippi River near St. Francisville, La. & 26,300 & $34,700,000$ & $72,500,000$ & $2,000,000$ & $206,000,000$ \\
\hline $3-14$ & Mississippi River below Belle Chasse, La. & 26,700 & $40,900,000$ & $72,300,000$ & $2,700,000$ & $207,000,000$ \\
\hline
\end{tabular}

Table 66. - Discharge and corresponding transport data for dissolved major anions in water samples collected from the Mississippi River and some of its tributaries during the May-June 1990 cruise

[ --, not determined; All results are in kilograms per day except discharge, which is in cubic meters per second ]

\begin{tabular}{|c|c|c|c|c|c|c|}
\hline $\begin{array}{l}\text { Date } \\
1990\end{array}$ & Sampling site & Discharge & Chloride & Sulfate & $\begin{array}{c}\text { Nitrate as } \\
\text { nitrogen }\end{array}$ & Bicarbonate \\
\hline 6-07 & Illinois River at Valley City, Ill. & 1,230 & $4,800,000$ & $8,000,000$ & - & $26,400,000$ \\
\hline $6-11$ & Mississippi River below Grafton, Ill. & 5,040 & $8,700,000$ & $16,000,000$ & $2,500,000$ & $70,700,000$ \\
\hline $6-13$ & Mississippi River at Thebes, III. & 12,600 & $13,000,000$ & $42,200,000$ & $3,100,000$ & $141,000,000$ \\
\hline 6-14 & Ohio River at Olmsted, Ill. & 9,550 & $6,900,000$ & $24,900,000$ & $1,300,000$ & $80,900,000$ \\
\hline 6-18 & Mississippi River below Memphis, Tenn. & 20,800 & $20,100,000$ & $67,200,000$ & $3,900,000$ & $215,000,000$ \\
\hline $6-20$ & Mississippi River below Arkansas City, Ark. & 25,500 & $24,600,000$ & $73,700,000$ & $4,200,000$ & $258,000,000$ \\
\hline $6-22$ & Yazoo River below Steele Bayou, Miss. & 1,250 & 360,000 & 690,000 & 60,000 & $5,000,000$ \\
\hline $6-23$ & Mississippi River below Vicksburg, Miss. & 27,300 & $26,500,000$ & $75,000,000$ & $4,200,000$ & $274,000,000$ \\
\hline $6-25$ & Mississippi River near St. Francisville, La. & 23,200 & $21,500,000$ & $63,800,000$ & $3,500,000$ & $232,000,000$ \\
\hline $6-27$ & Mississippi River below Belle Chasse, La. & 23,300 & $25,400,000$ & $65,600,000$ & $3,500,000$ & $232,000,000$ \\
\hline
\end{tabular}


Table 67.- Concentration data for dissolved trace elements in ultrafilter permeate from water samples collected from the Mississippi River and some of its tributaries during the July-August 1987 cruise

[<, less than detection limit; All results are in micrograms per liter, except iron and strontium which are in milligrams per liter ]

\begin{tabular}{|c|c|c|c|c|c|c|c|c|}
\hline $\begin{array}{l}\text { Date } \\
1987\end{array}$ & Sampling site & Aiuminum & Arsenic & Barium & Beryllium & Boron & Cadmium & Chromlum \\
\hline 7.21 & Mississippi River at Hartford, Ill. & 57 & 3.1 & 71 & $<0.02$ & 53 & 0.29 & 0.95 \\
\hline $7-22$ & Mississippi River at St. Louis, Mo. & 44 & 2.9 & 110 & $<0.02$ & 48 & 7.8 & 3.2 \\
\hline $7-23$ & Mississippi River at Chester, Ill. & 26 & 3.7 & 110 & 0.042 & 78 & 2.6 & 2.2 \\
\hline $7-26$ & $\begin{array}{l}\text { Ohio River below Smithland Locks and Dam, } \\
\text { Ill.-Ky. }\end{array}$ & 15 & 2.2 & 56 & 0.046 & 94 & 0.75 & 1.1 \\
\hline $7-27$ & Ohio River at Olmsted, III. & 8.1 & 2 & 42 & 0.027 & 61 & 0.59 & 0.43 \\
\hline $7-28$ & Mississippi River below Hickman, Ky. & 14 & 3.3 & 93 & 0.031 & 81 & 0.89 & 0.69 \\
\hline $7-30$ & Mississippi River at Helena, Ark. & 10 & 3 & 79 & 0.037 & 69 & 0.51 & 0.51 \\
\hline $7-31$ & White River at Mile 11.5, Ark. & 2.9 & 1.2 & 38 & 0.18 & 15 & 0.37 & 1.2 \\
\hline $8-01$ & Arkansas River at Mile 55.9, Ark. & 3.8 & 6.4 & 120 & 0.19 & 90 & 0.51 & 1.3 \\
\hline $8-02$ & Mississippi River above Arkansas City, Ark. & 2.4 & 2.3 & 76 & 0.19 & 60 & 0.47 & 0.72 \\
\hline 8-04 & Mississippi River below Vicksburg, Miss. & 2.2 & 3.2 & 73 & 0.2 & 69 & 0.46 & 8.2 \\
\hline $8-06$ & $\begin{array}{l}\text { Old River Outflow Channel near Knox } \\
\text { Landing, La. }\end{array}$ & 3.8 & 3.2 & 75 & 0.19 & 59 & 0.61 & 0.96 \\
\hline $8-07$ & Mississippi River near St. Francisville, La. & 4.6 & 3.1 & 76 & 0.18 & 60 & 1 & 0.74 \\
\hline $8-09$ & Mississippi River below Belle Chasse, La. & 9.1 & 3.8 & 76 & 0.2 & 66 & 0.58 & 0.83 \\
\hline
\end{tabular}

Table 67.-- Concentration data for dissolved trace elements in ultrafilter permeate from water samples collected from the Mississippi River and some of its tributaries during the July-August 1987 cruise - Continued

\begin{tabular}{|c|c|c|c|c|c|c|c|c|}
\hline $\begin{array}{l}\text { Date } \\
1987\end{array}$ & Sampling site & Cobalt & Copper & Iron & Lead & Lithium & Manganese & Molybdenum \\
\hline $7-21$ & Mississippi River at Hartford, Ill. & 0.068 & 2.1 & $<0.005$ & 0.49 & 6.8 & 3.8 & 2.9 \\
\hline 7.22 & Mississippi River at St. Louis, Mo. & $<0.008$ & 7.3 & $<0.005$ & 1.6 & 12 & 2 & 3.1 \\
\hline $7-23$ & Mississippi River at Chester, Ill. & 0.028 & 3 & $<0.005$ & 0.31 & 14 & 0.67 & 3.7 \\
\hline $7-26$ & $\begin{array}{l}\text { Ohio River below Smithland Locks and Dam, } \\
\text { Ill.-Ky. }\end{array}$ & 0.044 & 2.5 & $<0.005$ & 0.56 & 5.7 & 0.75 & 4.3 \\
\hline $7-27$ & Ohio River at Olmsted, Ill. & 0.15 & 2.1 & $<0.005$ & 0.68 & 3.6 & 0.24 & 3.5 \\
\hline $7-28$ & Mississippi River below Hickman, Ky. & $<0.008$ & 2.7 & $<0.005$ & 0.23 & 14 & 0.26 & 3.8 \\
\hline 7.30 & Mississippi River at Helena, Ark. & 0.026 & 3 & $<0.005$ & 0.29 & 9.2 & 0.48 & 3.4 \\
\hline $7-31$ & White River at Mile 11.5, Ark. & 0.14 & 1.2 & $<0.005$ & 0.14 & 0.68 & 0.51 & 1.2 \\
\hline $8-01$ & Arkansas River at Mile 55.9, Ark. & 0.28 & 4.2 & $<0.005$ & 0.25 & 10 & 0.91 & 1.9 \\
\hline $8-02$ & Mississippi River above Arkansas City, Ark. & 0.17 & 2.3 & $<0.005$ & 2.4 & 9.5 & 0.84 & 3 \\
\hline 8-04 & Mississippi River below Vicksburg, Miss. & 0.21 & 2.5 & $<0.005$ & 0.29 & 9.2 & 1 & 3 \\
\hline 8-06 & $\begin{array}{l}\text { Old River Outflow Channel near Knox } \\
\text { Landing, La. }\end{array}$ & 0.093 & 2.7 & $<0.005$ & 0.21 & 8.7 & 0.82 & 3 \\
\hline $8-07$ & Mississippi River near St. Francisville, La. & 0.2 & 2.7 & $<0.005$ & 0.15 & 8.8 & 0.84 & 3 \\
\hline 8-09 & Mississippi River below Belle Chasse, La. & 0.27 & 3 & $<0.005$ & 0.51 & 9.9 & 2 & 3.1 \\
\hline
\end{tabular}


Table 67.- Concentration data for dissolved trace elements in ultrafilter permeate from water samples collected from the Mississippi River and some of its tributaries during the July-August 1987 cruise - Continued

\begin{tabular}{|c|c|c|c|c|c|c|c|}
\hline $\begin{array}{l}\text { Date } \\
1987\end{array}$ & Sampling site & Nickel & Strontium & Thallium & Uranium & Vanadium & Zinc \\
\hline $7-21$ & Mississippi River at Hartford, Ill. & $<0.03$ & 0.13 & 0.2 & 1.7 & 4.1 & 1.8 \\
\hline $7-22$ & Mississippi River at St. Louis, Mo. & 3.5 & 0.22 & 0.2 & 2.1 & 3.7 & 26 \\
\hline $7-23$ & Mississippi River at Chester, Ill. & 2.6 & 0.21 & 0.41 & 3.1 & 5.2 & 3.6 \\
\hline $7-26$ & $\begin{array}{l}\text { Ohio River below Smithland Locks and Dam, } \\
\text { IIl.-Ky. }\end{array}$ & 0.99 & 0.23 & 0.47 & 0.85 & 2.7 & 2.2 \\
\hline $7-27$ & Ohio River at Olmsted, IIl. & 2.5 & 0.16 & 0.45 & 0.61 & 2.1 & 2.1 \\
\hline $7-28$ & Mississippi River below Hickman, Ky. & 1.1 & 0.23 & 0.47 & 2.8 & 4.5 & 2.1 \\
\hline $7-30$ & Mississippi River at Helena, Ark. & 1.8 & 0.2 & 0.46 & 1.9 & 4.1 & 4 \\
\hline $7-31$ & White River at Mile 11.5, Ark. & $<0.03$ & 0.04 & $<0.05$ & 0.47 & 1.8 & 0.38 \\
\hline 8-01 & Arkansas River at Mile 55.9, Ark. & $<0.03$ & 0.5 & $<0.05$ & 2.1 & 15 & 1.8 \\
\hline $8-02$ & Mississippi River above Arkansas City, Ark. & $<0.03$ & 0.23 & $<0.05$ & 2.5 & $<0.07$ & 0.69 \\
\hline $8-04$ & Mississippi River below Vicksburg, Miss. & $<0.03$ & 0.22 & $<0.05$ & 3.3 & 4.8 & 2 \\
\hline $8-06$ & $\begin{array}{l}\text { Old River Outflow Channel near Knox } \\
\text { Landing, La. }\end{array}$ & $<0.03$ & 0.23 & 0.055 & 1.5 & 4.7 & 1.2 \\
\hline 8-07 & Mississippi River near St. Francisville, La. & $<0.03$ & 0.23 & $<0.05$ & 1.3 & 4.9 & 1.4 \\
\hline 8-09 & Mississippi River below Belle Chasse, La. & 1.3 & 0.24 & $<0.05$ & 0.93 & 7.4 & 2 \\
\hline
\end{tabular}

Table 68.- Concentration data for dissolved trace elements in ultrafilter permeate from water samples collected from the Mississippi River and some of its tributaries during the NovemberDecember 1987 cruise

$[<$, less than detection limit; All results are in micrograms per liter, except iron and strontium which are in milligrams per liter ]

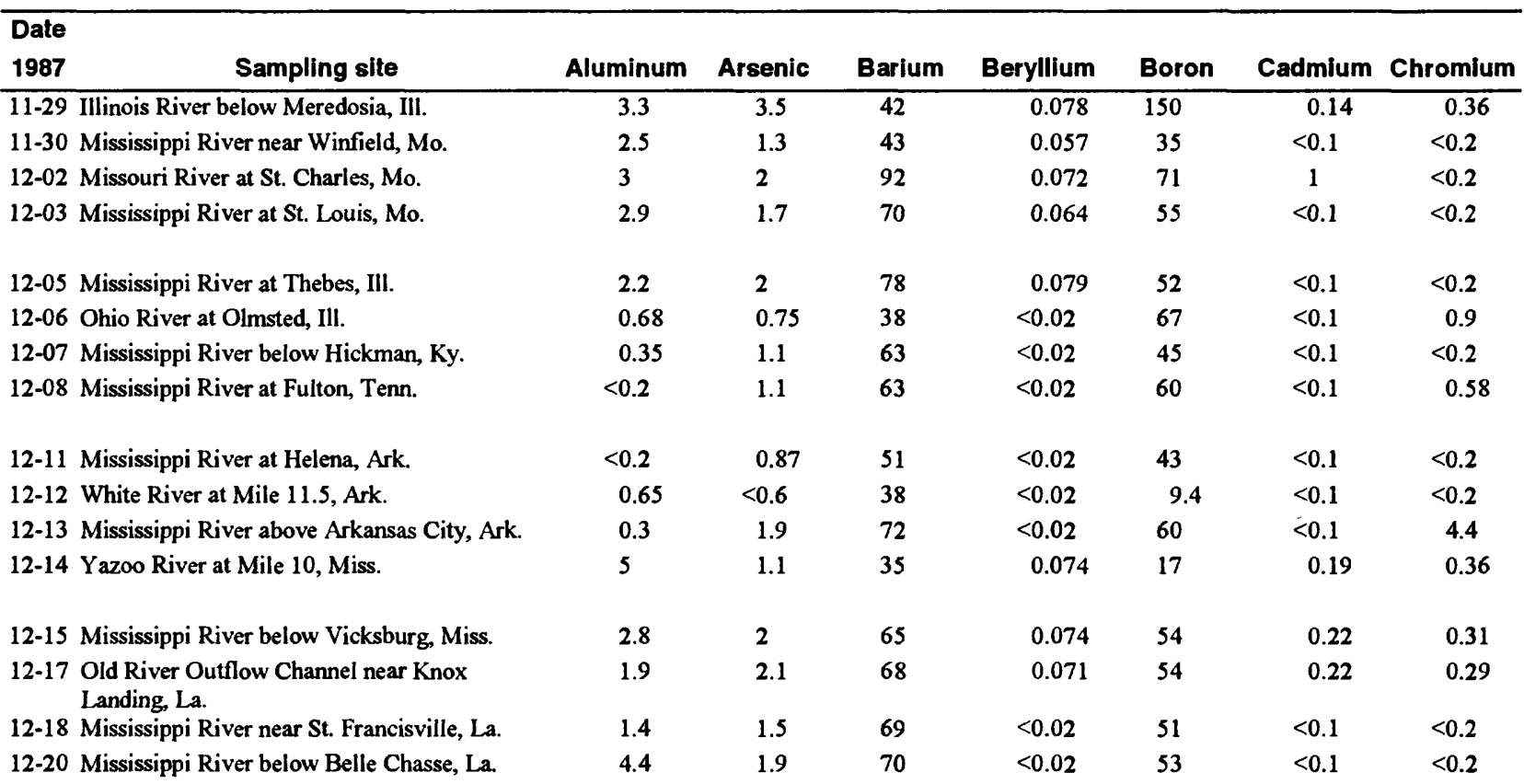


Table 68.-- Concentration data for dissolved trace elements in ultrafilter permeate from water samples collected from the Mississippi River and some of its tributaries during the NovemberDecember 1987 cruise - Continued

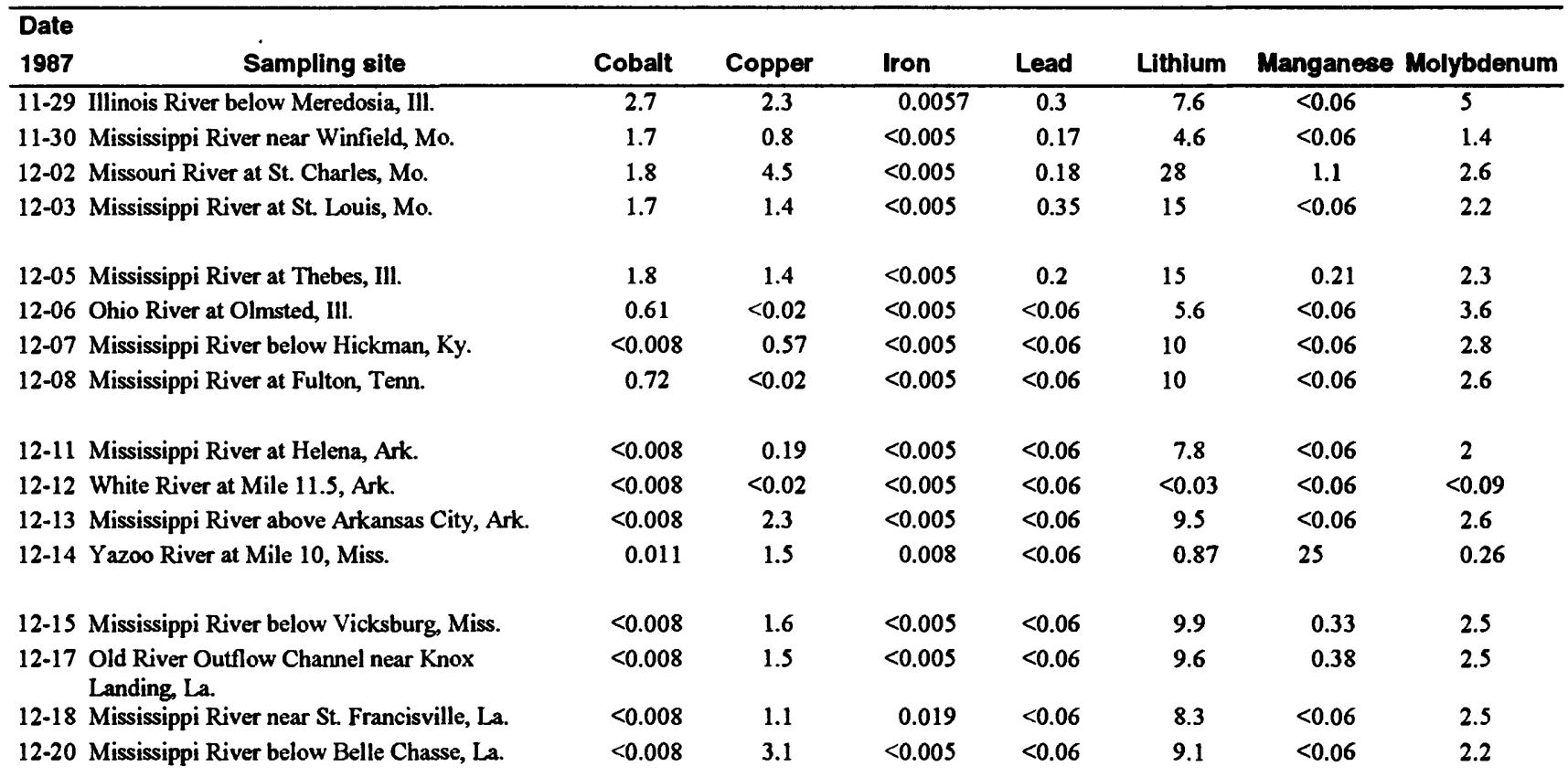

Table 68.-- Concentration data for dissolved trace elements in ultrafilter permeate from water samples collected from the Mississippi River and some of its tributaries during the NovemberDecember 1987 cruise -- Continued

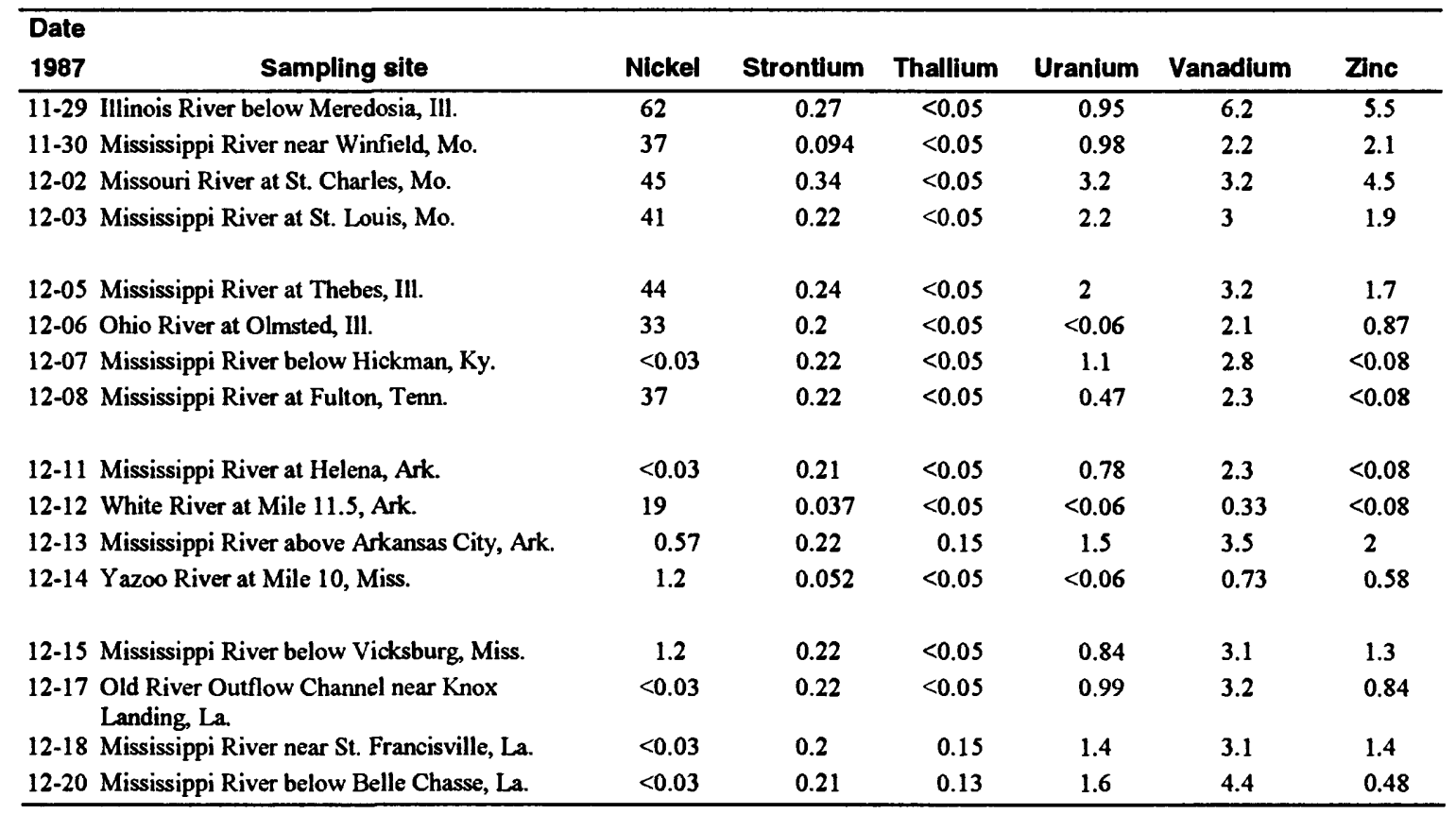


Table 69.- Concentration data for dissolved trace elements in ultrafilter permeate from water samples collected from the Mississippi River and some of its tributaries during the May-June 1988 cruise

[ <, less than detection limit; All results are in micrograms per liter, except iron and strontium which are in milligrams per liter ]

\begin{tabular}{|c|c|c|c|c|c|c|c|c|}
\hline $\begin{array}{l}\text { Date } \\
1988\end{array}$ & Sampling site & Aluminum & Arsenic & Barlum & Berylllum & Boron & Cadmlum & Chromium \\
\hline $5-16$ & Illinois River below Meredosia, Ill. & 2 & 3.9 & 62 & 0.13 & 110 & 0.98 & 3.9 \\
\hline 5-17 & Mississippi River near Winfield, Mo. & 2.7 & 1.5 & 54 & 0.07 & 23 & 5.8 & 1.4 \\
\hline $5-19$ & Missouri River at Hermann, Mo. & 2.5 & 2.9 & 100 & 0.11 & 74 & 1.2 & 1.8 \\
\hline $5-20$ & Mississippi River at St. Louis, Mo. & 2.6 & 2.4 & 86 & 0.047 & 66 & 1.2 & $<0.2$ \\
\hline $5-22$ & Mississippi River at Thebes, Ill. & 2.3 & 2.4 & 83 & 0.059 & 55 & 0.71 & $<0.2$ \\
\hline $5-23$ & Ohio River at Olmsted, Ill. & 5.7 & 1.1 & 38 & 0.063 & 41 & 1.2 & 0.37 \\
\hline $5-24$ & Mississippi River below Hickman, Ky. & 7.7 & 2.2 & 61 & 0.6 & 51 & 1.2 & 1 \\
\hline $5-26$ & Mississippi River at Fulton, Tenn. & 2.3 & 2.1 & 64 & 0.57 & 49 & 1.5 & 1 \\
\hline $5-28$ & Mississippi River at Helena, Ark. & 2.7 & 2.2 & 65 & 0.58 & 49 & 1.4 & 1.2 \\
\hline $5-29$ & White River at Mile 11.5, Ark. & 3.4 & 1.1 & 36 & 0.61 & 10 & 0.78 & 1.5 \\
\hline $5-29$ & Arkansas River at Mile 1.0, Ark. & 4.2 & 1.8 & 57 & 0.36 & 42 & 0.83 & 0.6 \\
\hline $5-30$ & Mississippi River above Arkansas City, Ark. & 4.8 & 1.4 & 76 & 0.38 & 17 & 0.52 & 1.5 \\
\hline $6-02$ & Mississippi River below Vicksburg, Miss. & 4 & 1.8 & 60 & 0.4 & 46 & 0.77 & 1.7 \\
\hline $6-04$ & $\begin{array}{l}\text { Old River Outflow Channel near Knox } \\
\text { Landing, La. }\end{array}$ & 2.5 & 1.4 & 67 & $<0.02$ & 47 & 0.38 & 2.3 \\
\hline $6-05$ & Mississippi River near St. Francisville, La. & 3.5 & 1.6 & 74 & $<0.02$ & 45 & 0.25 & 1.5 \\
\hline $6-07$ & Mississippi River below Belle Chasse, La. & 14 & 1.7 & 72 & $<0.02$ & 40 & 0.23 & 2.2 \\
\hline
\end{tabular}

Table 65.- Concentration data for dissolved trace elements in ultrafilter permeate from water samples collected from the Mississippi River and some of its tributaries during the May-June 1988 cruise - Continued

\begin{tabular}{|c|c|c|c|c|c|c|c|c|}
\hline $\begin{array}{l}\text { Date } \\
1988\end{array}$ & Sampllng site & Cobalt & Copper & Iron & Lead & Lithlum & Manganese & Molybdenum \\
\hline $5-16$ & Illinois River below Meredosia, Ill. & 0.43 & 2 & $<0.005$ & 0.67 & 6.8 & 0.29 & 6.1 \\
\hline 5.17 & Mississippi River near Winfield, Mo. & 0.19 & 1.3 & $<0.005$ & 0.3 & 6.3 & 0.36 & 1.3 \\
\hline $5-19$ & Missouri River at Hermann, Mo. & $<0.008$ & 1.8 & $<0.005$ & 0.25 & 33 & 0.63 & 3.1 \\
\hline $5-20$ & Mississippi River at St. Louis, Mo. & 0.18 & 1.2 & $<0.005$ & $<0.06$ & 25 & 0.73 & 3.2 \\
\hline $5-22$ & Mississippi River at Thebes, Ill. & $<0.008$ & 1.1 & $<0.005$ & $<0.06$ & 18 & 0.38 & 2.9 \\
\hline $5-23$ & Ohio River at Olmsted, Ill. & $<0.008$ & 1.4 & $<0.005$ & 0.12 & 4.7 & 0.44 & 2.8 \\
\hline $5-24$ & Mississippi River below Hickman, Ky. & 0.49 & 1.8 & $<0.005$ & 0.081 & 12 & 1.2 & 2.5 \\
\hline $5-26$ & Mississippi River at Fulton, Tenn. & 0.39 & 1.7 & $<0.005$ & 0.069 & 11 & 1.2 & 2.5 \\
\hline $5-28$ & Mississippi River at Helena, Ark. & 0.36 & 1.9 & $<0.005$ & 0.12 & 11 & 1.4 & 2.5 \\
\hline $5-29$ & White River at Mile 11.5, Ark. & 0.37 & 0.77 & $<0.005$ & $<0.06$ & 0.91 & 1.1 & 0.92 \\
\hline $5-29$ & Arkansas River at Mile 1.0, Ark. & $<0.008$ & 1.8 & $<0.005$ & 0.5 & 9 & 0.48 & 2.3 \\
\hline $5-30$ & Mississippi River above Arkansas City, Ark. & $<0.008$ & 1.3 & $<0.005$ & 0.31 & 2.8 & 90 & 1.4 \\
\hline $6-02$ & Mississippi River below Vicksburg, Miss. & $<0.008$ & 1.7 & $<0.005$ & 0.75 & 9.5 & 0.35 & 2.4 \\
\hline $6-04$ & $\begin{array}{l}\text { Old River Outflow Channel near Knox } \\
\text { Landing, La. }\end{array}$ & $<0.008$ & 1.1 & $<0.005$ & $<0.06$ & 10 & $<0.06$ & 2.6 \\
\hline 6-05 & Mississippi River near St. Francisville, La. & $<0.008$ & 1 & $<0.005$ & $<0.06$ & 9.9 & $<0.06$ & 2.7 \\
\hline $6-07$ & Mississippi River below Belle Chasse, La. & $<0.008$ & 1.2 & $<0.005$ & $<0.06$ & 9.6 & 0.086 & 2.7 \\
\hline
\end{tabular}


Table 69.- Concentration data for dissolved trace elements in ultrafilter permeate from water samples collected from the Mississippi River and some of its tributaries during the May-June 1988 cruise - Continued

\begin{tabular}{|c|c|c|c|c|c|c|c|}
\hline $\begin{array}{l}\text { Date } \\
1988\end{array}$ & Sampling site & Nickel & Strontium & Thallium & Uranium & Vanadium & Zinc \\
\hline $5-16$ & Illinois River below Meredosia, III. & 13 & 0.2 & $<0.05$ & 1.8 & 7.7 & 2 \\
\hline $5-17$ & Mississippi River near Winfield, Mo. & 3.3 & 0.096 & $<0.05$ & 2.1 & 2.5 & 1.7 \\
\hline 5.19 & Missouri River at Hermann, Mo. & 0.96 & 0.44 & $<0.05$ & 5.1 & 4.2 & 1.4 \\
\hline $5-20$ & Mississippi River at St. Louis, Mo. & 2.2 & 0.3 & 0.31 & 4.1 & 4.1 & $<0.08$ \\
\hline $5-22$ & Mississippi River at Thebes, Ill. & $<0.03$ & 0.27 & 0.35 & 3.6 & 4 & $<0.08$ \\
\hline $5-23$ & Ohio River at Olmsted, Ill. & $<0.03$ & 0.19 & 0.38 & 0.97 & 2.2 & 0.9 \\
\hline $5-24$ & Mississippi River below Hickman, $\mathrm{Ky}$. & 2 & 0.22 & $<0.05$ & 2 & 3.1 & 1.2 \\
\hline $5-26$ & Mississippi River at Fulton, Tenn. & 0.47 & 0.22 & $<0.05$ & 2.2 & 2.9 & 1.4 \\
\hline $5-28$ & Mississippi River at Helena, Ark. & $<0.03$ & 0.22 & $<0.05$ & 2.3 & 2.9 & 1.4 \\
\hline $5-29$ & White River at Mile 11.5, Ark. & $<0.03$ & 0.042 & $<0.05$ & 0.56 & 0.92 & 0.28 \\
\hline $5-29$ & Arkansas River at Mile 1.0, Ark. & $<0.03$ & 0.21 & 0.13 & 2 & 2.5 & 1.9 \\
\hline $5-30$ & Mississippi River above Arkansas City, Ark. & $<0.03$ & 0.12 & 0.1 & 0.62 & 1.5 & 0.34 \\
\hline $6-02$ & Mississippi River below Vicksburg, Miss. & $<0.03$ & 0.22 & 0.14 & 2.1 & 2.7 & 1.1 \\
\hline $6-04$ & $\begin{array}{l}\text { Old River Outflow Channel near Knox } \\
\text { Landing, La. }\end{array}$ & $<0.03$ & 0.22 & $<0.05$ & 1.7 & 3.1 & $<0.08$ \\
\hline $6-05$ & Mississippi River near St. Francisville, La. & $<0.03$ & 0.21 & $<0.05$ & 1.8 & 3.2 & $<0.08$ \\
\hline 6-07 & Mississippi River below Belle Chasse, La. & $<0.03$ & 0.22 & $<0.05$ & 1.9 & 4.3 & $<0.08$ \\
\hline
\end{tabular}

Table 70.- Concentration data for dissolved trace elements in ultrafilter permeate from water samples collected from the Mississippi River and some of its tributaries during the March-April 1989 cruise

$[<$, less than detection limit; All results are in micrograms per liter, except iron and strontium which are in milligrams per liter ]

\begin{tabular}{|c|c|c|c|c|c|c|c|c|}
\hline $\begin{array}{l}\text { Date } \\
1989\end{array}$ & Sampling site & Aluminum & Arsenic & Barium & Beryllium & Boron & Cadmium & Chromium \\
\hline 3-09 & Illinois River at Hardin, III. & 1.6 & 1.7 & 50 & $<0.02$ & 130 & 1.3 & 0.67 \\
\hline $3-10$ & Mississippi River near Winfield, Mo. & 0.5 & 0.98 & 45 & $<0.02$ & 36 & $<0.1$ & 0.55 \\
\hline $3-12$ & Missouri River at Hermann, Mo. & 3.8 & 1.8 & 62 & $<0.02$ & 45 & 0.81 & $<0.2$ \\
\hline $3-13$ & Mississippi River at St. Louis, Mo. & 2.8 & 1.5 & 54 & $<0.02$ & 58 & $<0.1$ & 0.46 \\
\hline $3-15$ & Mississippi River at Thebes, Ill. & 2.5 & 1.5 & 58 & $<0.02$ & 53 & $<0.1$ & $<0.2$ \\
\hline $3-16$ & Ohio River at Olmsted, Ill. & 5 & $<0.6$ & 26 & $<0.02$ & 14 & 0.22 & $<0.2$ \\
\hline $3-17$ & Mississippi River below Hickman, Ky. & 7.6 & $<0.6$ & 34 & $<0.02$ & 22 & $<0.1$ & $<0.2$ \\
\hline 3-19 & Mississippi River below Fulton, Tenn. & 7.2 & $<0.6$ & 35 & $<0.02$ & 24 & 0.12 & $<0.2$ \\
\hline $3-21$ & Mississippi River at Helena, Ark. & 4.8 & $<0.6$ & 39 & $<0.02$ & 22 & $<0.1$ & $<0.2$ \\
\hline $3-22$ & White River at Mile 11.5, Ark. & 3.7 & 0.85 & 31 & 0.047 & 5.3 & $<0.1$ & $<0.2$ \\
\hline $3-23$ & Arkansas River at Pendleton, Ark. & 4 & 1 & 50 & $<0.02$ & 25 & 0.12 & $<0.2$ \\
\hline 3-24 & Mississippi River above Arkansas City, Ark. & 4.8 & 1 & 43 & $<0.02$ & 24 & $<0.1$ & $<0.2$ \\
\hline 3-26 & Yazoo River below Steele Bayou, Miss. & 5.4 & 1.7 & 26 & $<0.02$ & 12 & $<0.1$ & $<0.2$ \\
\hline 3-27 & Mississippi River below Vicksburg, Miss. & 3.8 & 1.4 & 46 & $<0.02$ & 24 & $<0.1$ & $<0.2$ \\
\hline $3-29$ & $\begin{array}{l}\text { Old River Outflow Channel near Knox } \\
\text { Landing, La. }\end{array}$ & 2.2 & 1.2 & 47 & $<0.02$ & 21 & $<0.1$ & $<0.2$ \\
\hline 3-30 & Mississippi River near St. Francisville, La. & 2 & 0.84 & 48 & $<0.02$ & 20 & $<0.1$ & $<0.2$ \\
\hline 4-01 & Mississippi River below Belle Chasse, La. & 2.5 & 1 & 50 & $<0.02$ & 22 & $<0.1$ & $<0.2$ \\
\hline
\end{tabular}


Table 70.- Concentration data for dissolved trace elements in ultrafilter permeate from water samples collected from the Mississippi River and some of its tributaries during the March-April 1989 cruise - Continued

\begin{tabular}{|c|c|c|c|c|c|c|c|c|}
\hline $\begin{array}{l}\text { Date } \\
1989\end{array}$ & Sampling site & Cobalt & Copper & Iron & Lead & Lithium & Manganese & Molybdenum \\
\hline 3-09 & Illinois River at Hardin, Ill. & 0.75 & 2.3 & $<0.005$ & 0.39 & 6.6 & 19 & 6.1 \\
\hline 3.10 & Mississippi River near Winfield, Mo. & 0.45 & 1.3 & $<0.005$ & 0.11 & 3.1 & 2.4 & 1.4 \\
\hline 3-12 & Missouri River at Hermann, Mo. & 0.31 & 1.3 & $<0.005$ & $<0.06$ & 13 & 4.2 & 1.5 \\
\hline 3-13 & Mississippi River at St. Louis, Mo. & 0.48 & 1.4 & $<0.005$ & 0.18 & 7.5 & 2.5 & 2.5 \\
\hline $3-15$ & Mississippi River at Thebes, Ill. & 0.42 & 1.2 & $<0.005$ & $<0.06$ & 7.7 & 1.5 & 2 \\
\hline 3-16 & Ohio River at Olmsted, III. & 0.2 & 0.52 & 0.0072 & $<0.06$ & 1.6 & 3.6 & 0.64 \\
\hline 3-17 & Mississippi River below Hickman, Ky. & 0.23 & 2 & $<0.005$ & $<0.06$ & 2.6 & 1.7 & 0.91 \\
\hline 3-19 & Mississippi River below Fulton, Tenn. & 0.24 & 1.1 & $<0.005$ & $<0.06$ & 2.6 & 0.44 & 0.99 \\
\hline $3-21$ & Mississippi River at Helena, Ark. & 0.21 & 0.88 & 0.0051 & $<0.06$ & 2.3 & 0.48 & 0.94 \\
\hline 3-22 & White River at Mile 11.5, Ark. & 0.13 & 0.41 & 0.0086 & $<0.06$ & 0.24 & 3.8 & 0.22 \\
\hline $3-23$ & Arkansas River at Pendleton, Ark. & 0.13 & 0.54 & 0.0098 & $<0.06$ & 2.2 & 2.6 & 0.5 \\
\hline 3-24 & Mississippi River above Arkansas City, Ark. & 0.18 & 0.47 & $<0.005$ & $<0.06$ & 2.6 & 0.6 & 0.97 \\
\hline $3-26$ & Yazoo River below Steele Bayou, Miss. & 0.031 & 0.7 & 0.017 & $<0.06$ & 0.82 & 12 & 0.21 \\
\hline 3-27 & Mississippi River below Vicksburg, Miss. & 0.18 & 1 & $<0.005$ & $<0.06$ & 3.1 & 0.63 & 1.1 \\
\hline 3-29 & $\begin{array}{l}\text { Old River Outflow Channel near Knox } \\
\text { Landing, La. }\end{array}$ & 0.16 & 1.2 & 0.0062 & $<0.06$ & 2.8 & 3.1 & 0.94 \\
\hline $3-30$ & Mississippi River near St. Francisville, La. & 0.12 & 0.9 & $<0.005$ & $<0.06$ & 2.8 & 1.7 & 1 \\
\hline 4-01 & Mississippi River below Belle Chasse, La. & 0.1 & 0.89 & $<0.005$ & $<0.06$ & 2.8 & 3 & 0.94 \\
\hline
\end{tabular}

Table 70.- Concentration data for dissolved trace elements in ultrafilter permeate from water samples collected from the Mississippi River and some of its tributaries during the March-April 1989 cruise - Continued

\begin{tabular}{|c|c|c|c|c|c|c|c|}
\hline $\begin{array}{l}\text { Date } \\
1989\end{array}$ & Sampling site & Nickel & Strontium & Thallium & Uranium & Vanadium & Zinc \\
\hline $3-09$ & Illinois River at Hardin, Ill. & 18 & 0.27 & $<0.05$ & 1.7 & 1.4 & 9.2 \\
\hline $3-10$ & Mississippi River near Winfield, Mo. & 8.6 & 0.093 & $<0.05$ & 0.78 & 0.56 & 2 \\
\hline $3-12$ & Missouri River at Hermann, Mo. & 6.9 & 0.24 & $<0.05$ & 3.2 & 0.93 & 1.6 \\
\hline $3-13$ & Mississippi River at St. Louis, Mo. & 9.6 & 0.19 & $<0.05$ & 1.8 & 0.99 & 2.9 \\
\hline $3-15$ & Mississippi River at Thebes, Ill. & 8.3 & 0.19 & $<0.05$ & 1.9 & 1 & 2.6 \\
\hline $3-16$ & Ohio River at Olmsted, Ill. & 4.4 & 0.1 & $<0.05$ & 0.23 & 0.3 & 0.6 \\
\hline $3-17$ & Mississippi River below Hickman, Ky. & 5.1 & 0.12 & $<0.05$ & 0.49 & 0.36 & 0.44 \\
\hline $3-19$ & Mississippi River below Fulton, Tenn. & 5.4 & 0.12 & $<0.05$ & 0.47 & 0.45 & 0.44 \\
\hline $3-21$ & Mississippi River at Helena, Ark. & 4.8 & 0.12 & $<0.05$ & 0.43 & 0.44 & 0.35 \\
\hline 3-22 & White River at Mile 11.5, Ark. & 2.7 & 0.027 & $<0.05$ & $<0.06$ & 0.3 & 0.46 \\
\hline 3.23 & Arkansas River at Pendleton, Ark. & 3.8 & 0.17 & $<0.05$ & 0.56 & 0.39 & 0.78 \\
\hline $3-24$ & Mississippi River above Arkansas City, Ark. & 5.3 & 0.12 & $<0.05$ & 0.49 & 0.45 & 2.6 \\
\hline $3-26$ & Yazoo River below Steele Bayou, Miss. & 2.2 & 0.036 & $<0.05$ & $<0.06$ & 0.39 & 0.47 \\
\hline $3-27$ & Mississippi River below Vicksburg, Miss. & 5.3 & 0.12 & $<0.05$ & 0.63 & 0.75 & 1.5 \\
\hline 3-29 & $\begin{array}{l}\text { Old River Outflow Channel near Knox } \\
\text { Landing, La. }\end{array}$ & 5 & 0.12 & $<0.05$ & 0.72 & 0.64 & 1.1 \\
\hline 3-30 & Mississippi River near St. Francisville, La. & 5 & 0.12 & $<0.05$ & 0.75 & 0.6 & 2.7 \\
\hline $4-01$ & Mississippi River below Belle Chasse, La. & 5.1 & 0.12 & $<0.05$ & 0.64 & 0.76 & 0.82 \\
\hline
\end{tabular}


Table 71.- Concentration data for dissolved trace elements in ultrafilter permeate from water samples collected from the Mississippi River and some of its tributaries during the June 1989 cruise

$[<$, less than detection limit; All results are in micrograms per liter, except iron and strontium which are in milligrams per liter ]

\begin{tabular}{|c|c|c|c|c|c|c|c|c|}
\hline $\begin{array}{l}\text { Date } \\
1989\end{array}$ & Sampling site & Aluminum & Arsenic & Barium & Beryllium & Boron & Cadmium & Chromium \\
\hline $6-04$ & Illinois River at Hardin, Ill. & 7.2 & 2.9 & 65 & 0.038 & 150 & 0.34 & 0.42 \\
\hline $6-05$ & Mississippi River near Winfield, Mo. & 5.7 & 2.2 & 63 & $<0.02$ & 45 & $<0.1$ & 0.38 \\
\hline $6-07$ & Missouri River at Hermann, Mo. & 7.7 & 1.9 & 87 & 0.023 & 100 & $<0.1$ & 0.26 \\
\hline $6-08$ & Mississippi River at St. Louis, Mo. & 6.7 & 2 & 74 & $<0.02$ & 92 & $<0.1$ & 0.25 \\
\hline $6-10$ & Mississippi River at Thebes, Ill. & 5.7 & 2.5 & 78 & 0.045 & 96 & $<0.1$ & 0.28 \\
\hline $6-11$ & Ohio River at Olmsted, IIl. & 6.7 & 0.92 & 41 & $<0.02$ & 32 & $<0.1$ & $<0.2$ \\
\hline $6-12$ & Mississippi River below Hickman, Ky. & 4.7 & 1.4 & 55 & $<0.02$ & 47 & $<0.1$ & $<0.2$ \\
\hline $6-14$ & Mississippi River at Fulton, Tenn. & 8.6 & 1.4 & 53 & $<0.02$ & 43 & $<0.1$ & $<0.2$ \\
\hline $6-17$ & Mississippi River at Helena, Ark. & 5.1 & 1.3 & 53 & $<0.02$ & 38 & $<0.1$ & $<0.2$ \\
\hline 6-18 & White River at Mile 11.5, Ark. & 610 & 1.3 & 43 & $<0.02$ & 7.4 & 0.48 & $<0.2$ \\
\hline $6-20$ & Mississippi River above Arkansas City, Ark. & 5.2 & 1.6 & 49 & 0.042 & 32 & $<0.1$ & $<0.2$ \\
\hline 6-22 & Yazoo River below Steele Bayou, Miss. & 5 & 2 & 26 & $<0.02$ & 15 & $<0.1$ & $<0.2$ \\
\hline $6-23$ & Mississippi River below Vicksburg, Miss. & 4 & 1.4 & 48 & $<0.02$ & 32 & $<0.1$ & $<0.2$ \\
\hline $6-25$ & $\begin{array}{l}\text { Old River Outflow Channel near Knox } \\
\text { Landing, La. }\end{array}$ & 7 & 1.3 & 47 & $<0.02$ & 31 & 0.85 & $<0.2$ \\
\hline 6-26 & Mississippi River near St. Francisville, La. & 3.6 & 1.5 & 49 & $<0.02$ & 32 & $<0.1$ & $<0.2$ \\
\hline $6-28$ & Mississippi River below Belle Chasse, La. & 6 & 1.9 & 51 & 0.024 & 32 & $<0.1$ & $<0.2$ \\
\hline
\end{tabular}

Table 71.- Concentration data for dissolved trace elements in ultrafilter permeate from water samples collected from the Mississippi River and some of its tributaries during the June 1989 cruise -- Continued

\begin{tabular}{|c|c|c|c|c|c|c|c|c|}
\hline $\begin{array}{l}\text { Date } \\
1989\end{array}$ & Sampling site & Cobalt & Copper & Iron & Lead & Lithlum & Manganese & Molybdenum \\
\hline $6-04$ & Illinois River at Hardin, Ill. & 0.71 & 2.2 & $<0.005$ & $<0.06$ & 6.1 & 0.48 & 5.9 \\
\hline $6-05$ & Mississippi River near Winfield, Mo. & 0.41 & 1.4 & $<0.005$ & $<0.06$ & 4.8 & 0.46 & 1.7 \\
\hline 6-07 & Missouri River at Hermann, Mo. & 0.32 & 1.5 & $<0.005$ & $<0.06$ & 30 & 0.26 & 3.3 \\
\hline $6-08$ & Mississippi River at St. Louis, Mo. & 0.42 & 1.5 & $<0.005$ & $<0.06$ & 17 & 0.43 & 3 \\
\hline $6-10$ & Mississippi River at Thebes, $\mathrm{Ill}$. & 0.42 & 1.6 & $<0.005$ & $<0.06$ & 16 & 0.25 & 3.1 \\
\hline $6-11$ & Ohio River at Olmsted, Ill. & 0.1 & 1.3 & $<0.005$ & $<0.06$ & 2.2 & 0.19 & 2.1 \\
\hline $6-12$ & Mississippi River below Hickman, Ky. & 0.15 & 1.1 & $<0.005$ & $<0.06$ & 5.4 & 0.12 & 2.4 \\
\hline $6-14$ & Mississippi River at Fulton, Tenn. & 0.12 & 1.3 & $<0.005$ & 0.074 & 5.1 & 0.13 & 2.2 \\
\hline $6-17$ & Mississippi River at Helena, Ark. & 0.076 & 1.5 & $<0.005$ & $<0.06$ & 4.1 & 0.2 & 2.1 \\
\hline $6-18$ & White River at Mile 11.5, Ark. & 0.11 & 0.92 & 0.67 & 0.51 & 0.53 & 11 & 0.38 \\
\hline $6-20$ & Mississippi River above Arkansas City, Ark. & 0.23 & 1.3 & $<0.005$ & 0.076 & 3.4 & 0.31 & 1.8 \\
\hline $6-22$ & Yazoo River below Steele Bayou, Miss. & 0.056 & 2.1 & 0.011 & $<0.06$ & 0.66 & 4.5 & 0.24 \\
\hline $6-23$ & Mississippi River below Vicksburg, Miss. & 0.21 & 1.5 & $<0.005$ & $<0.06$ & 3 & 0.42 & 1.7 \\
\hline $6-25$ & $\begin{array}{l}\text { Old River Outflow Channel near Knox } \\
\text { Landing, La. }\end{array}$ & 0.19 & 1.9 & $<0.005$ & 0.091 & 3 & 0.79 & 1.8 \\
\hline $6-26$ & Mississippi River near St. Francisville, La. & 0.23 & 1.6 & $<0.005$ & $<0.06$ & 3 & 0.36 & 1.8 \\
\hline $6-28$ & Mississippi River below Belle Chasse, La. & 0.24 & 1.7 & $<0.005$ & $<0.06$ & 3.4 & 0.33 & 1.7 \\
\hline
\end{tabular}


Table 71.- Concentration data for dissolved trace elements in ultrafilter permeate from water samples collected from the Mississippi River and some of its tributaries during the June 1989 cruise - Continued

\begin{tabular}{|c|c|c|c|c|c|c|c|}
\hline $\begin{array}{l}\text { Date } \\
1989\end{array}$ & Sampling site & Nickel & Strontium & Thallium & Uranium & Vanadium & Zinc \\
\hline $6-04$ & Illinois River at Hardin, III. & 14 & 0.22 & $<0.05$ & 1.7 & 3.2 & 4.1 \\
\hline 6-05 & Mississippi River near Winfield, Mo. & 7.4 & 0.089 & $<0.05$ & 1.5 & 2.3 & 2.5 \\
\hline 6-07 & Missouri River at Hermann, Mo. & 7.8 & 0.4 & $<0.05$ & 3.8 & 2.4 & 3 \\
\hline 6-08 & Mississippi River at St. Louis, Mo. & 8.4 & 0.23 & 0.061 & 2.4 & 2.6 & 1.9 \\
\hline $6-10$ & Mississippi River at Thebes, III. & 8.6 & 0.22 & 0.073 & 2.3 & 2.7 & 1.6 \\
\hline $6-11$ & Ohio River at Olmsted, III. & 5.3 & 0.16 & $<0.05$ & 0.49 & 0.6 & 1.5 \\
\hline 6-12 & Mississippi River below Hickman, Ky. & 5.9 & 0.18 & $<0.05$ & 0.96 & 1.2 & 0.96 \\
\hline 6-14 & Mississippi River at Fulton, Tenn. & 5.9 & 0.18 & $<0.05$ & 0.95 & 1.1 & 1.5 \\
\hline $6-17$ & Mississippi River at Helena, Ark. & 5.1 & 0.19 & $<0.05$ & 0.72 & 1.1 & 0.97 \\
\hline $6-18$ & White River at Mile 11.5, Ark. & 3.9 & 0.043 & $<0.05$ & 0.3 & 1.5 & 4 \\
\hline $6-20$ & Mississippi River above Arkansas City, Ark. & 5.2 & 0.16 & 0.058 & 0.76 & 1.1 & 1.8 \\
\hline $6-22$ & Yazoo River below Steele Bayou, Miss. & 2.4 & 0.03 & $<0.05$ & $<0.06$ & 0.94 & 1.2 \\
\hline $6-23$ & Mississippi River below Vicksburg, Miss. & 5.2 & 0.15 & $<0.05$ & 0.59 & 1.1 & 0.99 \\
\hline 6-25 & $\begin{array}{l}\text { Old River Outflow Channel near Knox } \\
\text { Landing, La. }\end{array}$ & 4.7 & 0.15 & 0.099 & 0.78 & 1.2 & 1.6 \\
\hline $6-26$ & Mississippi River near St. Francisville, La. & 5.1 & 0.14 & $<0.05$ & 0.63 & 1.1 & 1.4 \\
\hline $6-28$ & Mississippi River below Belle Chasse, La. & 4.7 & 0.15 & $<0.05$ & 0.72 & 1.2 & 2.2 \\
\hline
\end{tabular}

Table 72.- Concentration data for dissolved trace elements in ultrafilter permeate from water samples collected from the Mississippi River and some of its tributaries during the FebruaryMarch 1990 cruise

$[<$, less than detection limit; All results are in micrograms per liter, except iron and strontium which are in milligrams per liter ]

\begin{tabular}{|c|c|c|c|c|c|c|c|c|}
\hline $\begin{array}{l}\text { Date } \\
1990\end{array}$ & Sampling site & Aluminum & Arsenic & Barlum & Beryllium & Boron & Cadmlum & Chromium \\
\hline $2-23$ & Cumberland River near Smithland, Ky. & 4.4 & $<0.6$ & 23 & $<0.02$ & 12 & 0.24 & $<0.2$ \\
\hline $2-24$ & Tennessee River near Calvert City, Ky. & 3.4 & $<0.6$ & 19 & $<0.02$ & 7.4 & $<0.1$ & 0.36 \\
\hline $2-25$ & Mississippi River near Cache, Ill. & 2.9 & 1.7 & 64 & $<0.02$ & 67 & $<0.1$ & 0.54 \\
\hline $2-28$ & Wabash River near New Haven, III. & 4 & 0.66 & 40 & $<0.02$ & 33 & $<0.1$ & $<0.2$ \\
\hline 3-01 & Ohio River at Uniontown, Ky. & 7 & $<0.6$ & 32 & 0.068 & 22 & 0.32 & 0.86 \\
\hline $3-03$ & Ohio River at Olmsted, Ill. & 4.5 & $<0.6$ & 27 & $<0.02$ & 18 & $<0.1$ & $<0.2$ \\
\hline 3-04 & Mississippi River below Hickman, Ky. & 3.9 & $<0.6$ & 35 & $<0.02$ & 24 & $<0.1$ & $<0.2$ \\
\hline $3-05$ & Mississippi River below Fulton, Tenn. & 4.2 & $<0.6$ & 35 & $<0.02$ & 23 & $<0.1$ & $<0.2$ \\
\hline 3-07 & Mississippi River at Helena, Ark. & 4 & 1.3 & 40 & $<0.02$ & 23 & 0.33 & $<0.2$ \\
\hline $3-08$ & Mississippi River above Arkansas City, Ark. & 3.6 & 1 & 40 & 0.027 & 21 & 0.37 & $<0.2$ \\
\hline $3-10$ & Mississippi River below Vicksburg, Miss. & 4.4 & 0.99 & 40 & $<0.02$ & 20 & 0.21 & $<0.2$ \\
\hline $3-12$ & Mississippi River near St. Francisville, La. & 4.2 & 1.1 & 42 & $<0.02$ & 21 & 0.23 & $<0.2$ \\
\hline $3-14$ & Mississippi River below Belle Chasse, La. & 3.5 & 1.1 & 43 & 0.031 & 18 & 0.33 & $<0.2$ \\
\hline
\end{tabular}


Table 72.- Concentration data for dissolved trace elements in ultrafilter permeate from water samples collected from the Mississippi River and some of its tributaries during the FebruaryMarch 1990 cruise - Continued

\begin{tabular}{|c|c|c|c|c|c|c|c|c|}
\hline $\begin{array}{l}\text { Date } \\
1990\end{array}$ & Sampling site & Cobalt & Copper & Iron & Lead & Lithium & Manganese & Molybdenum \\
\hline $2-23$ & Cumberland River near Smithland, Ky. & 0.28 & 1.4 & $<0.005$ & $<0.06$ & 0.77 & 1.4 & 0.85 \\
\hline $2-24$ & Tennessee River near Calvert City, Ky. & 0.18 & 1.2 & 0.0061 & $<0.06$ & 0.49 & 0.83 & 0.26 \\
\hline $2-25$ & Mississippi River near Cache, 111 . & 0.52 & 1.6 & $<0.005$ & $<0.06$ & 9.1 & 1.1 & 2.4 \\
\hline $2-28$ & Wabash River near New Haven, 111. & 0.48 & 1.6 & 0.013 & $<0.06$ & 1.4 & 4.1 & 1.8 \\
\hline 3-01 & Ohio River at Uniontown, Ky. & 0.33 & 1.7 & 0.0077 & $<0.06$ & 2.6 & 7.8 & 1.8 \\
\hline $3-03$ & Ohio River at Olmsted, 111. & 0.2 & 1.4 & $<0.005$ & 0.063 & 1.5 & 3.6 & 1 \\
\hline $3-04$ & Mississippi River below Hickman, Ky. & 0.25 & 1.3 & 0.0084 & 0.068 & 2.5 & 3 & 1.2 \\
\hline $3-05$ & Mississippi River below Fulton, Tenn. & 0.19 & 1.5 & 0.0084 & 0.095 & 2.5 & 2 & 1.1 \\
\hline $3-07$ & Mississippi River at Helena, Ark. & 0.29 & 1.5 & 0.0089 & $<0.06$ & 2.4 & 1.7 & 1.1 \\
\hline $3-08$ & Mississippi River above Arkansas City, Ark. & 0.25 & 2.4 & 0.0089 & $<0.06$ & 2.2 & 1.7 & 1.1 \\
\hline 3-10 & Mississippi River below Vicksburg, Miss. & 0.21 & 1.7 & $<0.005$ & $<0.06$ & 2 & 1.5 & 0.91 \\
\hline 3-12 & Mississippi River near St. Francisville, La. & 0.25 & 1.6 & $<0.005$ & $<0.06$ & 2.1 & 2.2 & 0.94 \\
\hline 3-14 & Mississippi River below Belle Chasse, La. & 0.22 & 1.5 & 0.0074 & $<0.06$ & 1.9 & 8.3 & 1 \\
\hline
\end{tabular}

Table 72.- Concentration data for dissolved trace elements in ultrafilter permeate from water samples collected from the Mississippi River and some of its tributaries during the FebruaryMarch 1990 cruise - Continued

\begin{tabular}{|c|c|c|c|c|c|c|c|}
\hline $\begin{array}{l}\text { Date } \\
1990\end{array}$ & Sampling site & Nickel & Strontium & Thallium & Uranium & Vanadium & Zinc \\
\hline $2-23$ & Cumberland River near Smithland, Ky. & 5.6 & 0.085 & $<0.05$ & 0.15 & 0.58 & 1.6 \\
\hline 2-24 & Tennessee River near Calvert City, Ky. & 3.2 & 0.039 & $<0.05$ & $<0.06$ & 0.54 & 1.2 \\
\hline $2-25$ & Mississippi River near Cache, 111. & 9.9 & 0.17 & $<0.05$ & 2 & 1.4 & 3 \\
\hline $2-28$ & Wabash River near New Haven, Ill. & 8.7 & 0.12 & $<0.05$ & 0.87 & 0.51 & 1.2 \\
\hline 3-01 & Ohio River at Uniontown, Ky. & 6.1 & 0.16 & 0.2 & 0.63 & 0.48 & 1.9 \\
\hline $3-03$ & Ohio River at Olmsted, III. & 5.4 & 0.1 & $<0.05$ & 0.41 & 0.25 & 1.4 \\
\hline 3.04 & Mississippi River below Hickman, Ky. & 5.5 & 0.12 & $<0.05$ & 0.6 & 0.42 & 1.5 \\
\hline 3.05 & Mississippi River below Fulton, Tenn. & 5.5 & 0.12 & $<0.05$ & 0.63 & 0.45 & 1.1 \\
\hline 3-07 & Mississippi River at Helena, Ark. & 5.9 & 0.13 & $<0.05$ & 0.56 & 0.5 & 1.1 \\
\hline 3-08 & Mississippi River above Arkansas City, Ark. & 5.1 & 0.1 & $<0.05$ & 0.64 & 0.55 & 1.2 \\
\hline $3-10$ & Mississippi River below Vicksburg, Miss. & 4.6 & 0.11 & $<0.05$ & 0.62 & 0.49 & 1.1 \\
\hline 3-12 & Mississippi River near St. Francisville, La. & 5.1 & 0.1 & $<0.05$ & 0.64 & 0.64 & 0.97 \\
\hline $3-14$ & Mississippi River below Belle Chasse, La. & 4.6 & 0.11 & $<0.05$ & 0.62 & 0.84 & 1.4 \\
\hline
\end{tabular}


Table 73. - Concentration data for dissolved trace elements in ultrafilter permeate from water samples collected from the Mississippi River and some of its tributaries during the May-June 1990 cruise

$[<$, less than detection limit; All results are in micrograms per liter, except iron and strontium which are in milligrams per liter ]

\begin{tabular}{|c|c|c|c|c|c|c|c|c|}
\hline $\begin{array}{l}\text { Date } \\
1990\end{array}$ & Sampling site & Aluminum & Arsenic & Barium & Beryllium & Boron & Cadmium & Chromium \\
\hline 6-07 & Illinois River at Valley City, Ill. & 4 & 0.8 & 64 & 0.065 & 86 & 0.72 & $<0.2$ \\
\hline $6-11$ & Mississippi River below Grafton, Ill. & 6.1 & 0.95 & 77 & 0.027 & 38 & $<0.1$ & $<0.2$ \\
\hline $6-13$ & Mississippi River at Thebes, Ill. & 6.1 & 1.4 & 77 & $<0.02$ & 38 & $<0.1$ & $<0.2$ \\
\hline $6-14$ & Ohio River at Olmsted, III. & 6.5 & $<0.6$ & 32 & $<0.02$ & 28 & $<0.1$ & $<0.2$ \\
\hline $6-18$ & Mississippi River below Memphis, Tenn. & 5.6 & 1.3 & 59 & $<0.02$ & 35 & 0.28 & $<0.2$ \\
\hline $6-20$ & Mississippi River below Arkansas City, Ark. & 5.5 & 0.87 & 58 & $<0.02$ & 31 & 0.11 & 1.1 \\
\hline $6-22$ & Yazoo River below Steele Bayou, Miss. & 5 & 3.9 & 33 & $<0.02$ & 30 & 0.17 & $<0.2$ \\
\hline $6-23$ & Mississippi River below Vicksburg, Miss. & 4.5 & 1.1 & 55 & $<0.02$ & 30 & $<0.1$ & $<0.2$ \\
\hline $6-25$ & Mississippi River near St. Francisville, La. & 4.3 & 0.89 & 57 & $<0.02$ & 35 & $<0.1$ & $<0.2$ \\
\hline $6-27$ & Mississippi River below Belle Chasse, La. & 7.5 & 1.4 & 60 & $<0.02$ & 35 & $<0.1$ & $<0.2$ \\
\hline
\end{tabular}

Table 73. - Concentration data for dissolved trace elements in ultrafilter permeate from water samples collected from the Mississippi River and some of its tributaries during the May-June 1990 cruise - Continued

\begin{tabular}{|c|c|c|c|c|c|c|c|c|}
\hline $\begin{array}{l}\text { Date } \\
1990\end{array}$ & Sampling site & Cobalt & Copper & Iron & Lead & Lithium & Manganese & Molybdenum \\
\hline 6-07 & Illinois River at Valley City, Ill. & 0.6 & 3.2 & $<0.005$ & $<0.06$ & 4.5 & 0.93 & 3.6 \\
\hline $6-11$ & Mississippi River below Grafton, Ill. & 0.37 & 2.1 & $<0.005$ & $<0.06$ & 3.4 & 0.58 & 1.8 \\
\hline $6-13$ & Mississippi River at Thebes, Ill. & 0.29 & 1.9 & $<0.005$ & $<0.06$ & 4.6 & 0.33 & 1.8 \\
\hline 6-14 & Ohio River at Olmsted, Ill. & 0.3 & 1.5 & $<0.005$ & $<0.06$ & 1.5 & 0.42 & 1.3 \\
\hline $6-18$ & Mississippi River below Memphis, Tenn. & 0.35 & 2.2 & $<0.005$ & $<0.06$ & 4 & 0.39 & 1.7 \\
\hline $6-20$ & Mississippi River below Arkansas City, Ark. & 0.27 & 1.8 & $<0.005$ & $<0.06$ & 3.4 & 0.26 & 1.6 \\
\hline $6-22$ & Yazoo River below Steele Bayou, Miss. & 0.15 & 3.4 & $<0.005$ & $<0.06$ & 1.6 & 69 & 0.58 \\
\hline $6-23$ & Mississippi River below Vicksburg, Miss. & 0.3 & 2 & $<0.005$ & $<0.06$ & 2.9 & 0.44 & 1.3 \\
\hline $6-25$ & Mississippi River near St. Francisville, La. & 0.23 & 1.9 & $<0.005$ & $<0.06$ & 3.1 & 0.51 & 1.6 \\
\hline $6-27$ & Mississippi River below Belle Chasse, La. & 0.48 & 2.3 & $<0.005$ & $<0.06$ & 3.6 & 0.59 & 1.4 \\
\hline
\end{tabular}

Table 73.- Concentration data for dissolved trace elements in ultrafilter permeate from water samples collected from the Mississippi River and some of its tributaries during the May-June 1990 cruise - Continued

\begin{tabular}{|c|c|c|c|c|c|c|c|}
\hline $\begin{array}{l}\text { Date } \\
1990\end{array}$ & Sampling site & Nickei & Strontium & Thallium & Uranium & Vanadium & Zinc \\
\hline 6-07 & Illinois River at Valley City, III. & 14 & 0.2 & $<0.05$ & 1.7 & 1.9 & 4.4 \\
\hline $6-11$ & Mississippi River below Grafton, Ill. & 8.2 & 0.13 & $<0.05$ & 1.6 & 1.8 & 1.9 \\
\hline $6-13$ & Mississippi River at Thebes, Ill. & 7.1 & 0.15 & $<0.05$ & 1.3 & 1.8 & 1.4 \\
\hline $6-14$ & Ohio River at Olmsted, Ill. & 5.8 & 0.12 & $<0.05$ & 0.29 & 0.57 & 1.2 \\
\hline $6-18$ & Mississippi River below Memphis, Tenn. & 6.9 & 0.14 & $<0.05$ & 0.74 & 1.3 & 2 \\
\hline $6-20$ & Mississippi River below Arkansas City, Ark. & 6.4 & 0.14 & $<0.05$ & 0.81 & 1.3 & 1 \\
\hline $6-22$ & Yazoo River below Steele Bayou, Miss. & 4.7 & 0.055 & $<0.05$ & $<0.06$ & 1.1 & 0.47 \\
\hline $6-23$ & Mississippi River below Vicksburg, Miss. & 6.4 & 0.14 & $<0.05$ & 0.62 & 1.3 & 2 \\
\hline $6-25$ & Mississippi River near St. Francisville, La. & 5.8 & 0.14 & $<0.05$ & 0.68 & 1.5 & 0.99 \\
\hline $6-27$ & Mississippi River below Belle Chasse, La. & 6.7 & 0.14 & $<0.05$ & 0.61 & 1.8 & 0.85 \\
\hline
\end{tabular}


Table 74. - Concentration data for dissolved major elements in ultrafilter permeate from water samples collected from the Mississippi River and some of its tributaries during the July-August 1987 cruise

[ All results are in milligrams per liter ]

\begin{tabular}{|c|c|c|c|c|c|c|}
\hline $\begin{array}{l}\text { Date } \\
1987\end{array}$ & Sampling site & Calcium & Magnesium & Potassium & Sodium & $\begin{array}{c}\text { Silicon } \\
\left(\mathrm{as} \mathrm{SiO}_{2}\right)\end{array}$ \\
\hline $7-21$ & Mississippi River at Hartford, Ill. & $\overline{42}$ & 19 & 3.4 & 15 & 4.5 \\
\hline $7-22$ & Mississippi River at St. Louis, Mo. & 44 & 15 & 4.9 & 24 & 7.5 \\
\hline $7-23$ & Mississippi River at Chester, Ill. & 44 & 15 & 4.8 & 23 & 7.7 \\
\hline $7-26$ & $\begin{array}{l}\text { Ohio River below Smithland Locks and Dam, } \\
\text { III.-Ky. }\end{array}$ & 46 & 13 & 3.7 & 17 & 4.7 \\
\hline 7.27 & Ohio River at Olmsted, Ill. & 34 & 8.2 & 2.8 & 12 & 3.1 \\
\hline $7-28$ & Mississippi River below Hickman, Ky. & 47 & 14 & 4.5 & 24 & 6.3 \\
\hline $7-30$ & Mississippi River at Helena, Ark. & 42 & 12 & 3.9 & 19 & 6.5 \\
\hline $7-31$ & White River at Mile 11.5, Ark. & 34 & 14 & 1.5 & 3.8 & 4.4 \\
\hline 8-01 & Arkansas River at Mile 55.9, Ark. & ss & 15 & 5.1 & 100 & 0.5 \\
\hline $8-02$ & Mississippi River above Arkansas City, Ark. & 43 & 13 & 4.1 & 29 & 5.9 \\
\hline 8-04 & Mississippi River below Vicksburg, Miss. & 43 & 13 & 4.1 & 26 & 6.1 \\
\hline $8-06$ & $\begin{array}{l}\text { Old River Outflow Channel near Knox } \\
\text { Landing, La. }\end{array}$ & 44 & 14 & 4.1 & 27 & 6.5 \\
\hline $8-07$ & Mississippi River near St. Francisville, La. & 44 & 14 & 4.1 & 27 & 6.6 \\
\hline $8-09$ & Mississippi River below Belle Chasse, La. & 43 & 14 & 5.9 & 37 & 6.7 \\
\hline
\end{tabular}

Table 75. - Concentration data for dissolved major elements in ultrafilter permeate from water samples collected from the Mississippi River and some of its tributaries during the NovemberDecember 1987 cruise

[ All results are in milligrams per liter ]

\begin{tabular}{|c|c|c|c|c|c|}
\hline Sampling site & Calcium & Magnesium & Potassium & Sodium & $\begin{array}{c}\text { Silicon } \\
\left(\mathrm{as} \mathrm{SiO}_{2}\right)\end{array}$ \\
\hline 11-29 Illinois River below Meredosia, Ill. & 65 & 29 & 4.5 & 44 & 1.9 \\
\hline 11-30 Mississippi River near Winfield, Mo. & 41 & 21 & 2.7 & 12 & 0.13 \\
\hline 12-02 Missouri River at St. Charles, Mo. & so & 17 & 4.6 & 39 & 8.8 \\
\hline 12-03 Mississippi River at St. Louis, Mo. & 46 & 19 & 3.8 & 26 & 4.7 \\
\hline 12-05 Mississippi River at Thebes, III. & 48 & 20 & 4 & 28 & 5.1 \\
\hline 12-06 Ohio River at Olmsted, Ill. & 37 & 11 & 3.2 & 24 & 3.8 \\
\hline 12-07 Mississippi River below Hickman, Ky. & 44 & 16 & 3.6 & 26 & 4.6 \\
\hline 12-08 Mississippi River at Fulton, Tenn. & 44 & 16 & 3.6 & 27 & 4.8 \\
\hline 12-11 Mississippi River at Helena, Ark. & 43 & 16 & 3.5 & 25 & 4.8 \\
\hline 12-12 White River at Mile 11.5, Ark. & 23 & 9.2 & 2.4 & 3.8 & 7.4 \\
\hline 12-13 Mississippi River above Arkansas City, Ark. & 42 & 16 & 3.5 & 29 & 5.3 \\
\hline 12-14 Yazoo River at Mile 10, Miss. & 8.6 & 2.9 & 3.3 & 6 & 7.3 \\
\hline 12-15 Mississippi River below Vicksburg, Miss. & 42 & 15 & 3.5 & 28 & 5.2 \\
\hline $\begin{array}{l}\text { 12-17 Old River Outflow Channel near Knox } \\
\text { Landing, La. }\end{array}$ & 42 & 15 & 3.5 & 29 & 5.3 \\
\hline 12-18 Mississippi River near St. Francisville, La. & 40 & 14 & 3.5 & 27 & 5 \\
\hline 12-20 Mississippi River below Belle Chasse, La. & 40 & 14 & 3.6 & 37 & 5.2 \\
\hline
\end{tabular}


Table 76. - Concentration data for dissolved major elements in ultrafilter permeate from water samples collected from the Mississippi River and some of its tributaries during the May-June 1988 cruise

[ All results are in milligrams per liter ]

\begin{tabular}{|c|c|c|c|c|c|c|}
\hline $\begin{array}{l}\text { Date } \\
1988 \\
\end{array}$ & Sampling site & Calcium & Magnesium & Potassium & Sodium & $\begin{array}{l}\text { Silicon } \\
\left(\text { as } \mathrm{SiO}_{2} \text { ) }\right.\end{array}$ \\
\hline $5-16$ & Illinois River below Meredosia, III. & 64 & 33 & 4 & 37 & 0.5 \\
\hline $5-17$ & Mississippi River near Winfield, Mo. & 35 & 21 & 3.3 & 11 & 0.066 \\
\hline $5-19$ & Missouri River at Hermann, Mo. & 59 & 20 & 7.3 & 50 & 8.7 \\
\hline $5-20$ & Mississippi River at St. Louis, Mo. & 51 & 21 & 4.9 & 35 & 5.2 \\
\hline $5-22$ & Mississippi River at Thebes, Ill. & 53 & 23 & 4.6 & 32 & 3.8 \\
\hline $5-23$ & Ohio River at Olmsted, Ill. & 36 & 12 & 3 & 17 & 0.32 \\
\hline $5-24$ & Mississippi River below Hickman, Ky. & 44 & 17 & 3.9 & 24 & 2.2 \\
\hline $5-26$ & Mississippi River at Fulton, Tenn. & 44 & 17 & 3.9 & 24 & 2 \\
\hline $5-28$ & Mississippi River at Helena, Ark. & 44 & 18 & 3.9 & 23 & 2.1 \\
\hline $5-29$ & White River at Mile 11.5, Ark. & 37 & 13 & 2.1 & 4 & 4.1 \\
\hline $5-29$ & Arkansas River at Mile 1.0, Ark. & 43 & 17 & 3.8 & 22 & 1.9 \\
\hline $5-30$ & Mississippi River above Arkansas City, Ark. & 26 & 8.2 & 3.4 & 13 & 11 \\
\hline $6-02$ & Mississippi River below Vicksburg, Miss. & 46 & 17 & 3.8 & 23 & 1.8 \\
\hline 6-04 & $\begin{array}{l}\text { Old River Outflow Channel near Knox } \\
\text { Landing, La. }\end{array}$ & 46 & 17 & 3.8 & 23 & 1.9 \\
\hline 6-05 & Mississippi River near St. Francisville, La. & 45 & 17 & 3.8 & 22 & 1.8 \\
\hline 6-07 & Mississippi River below Belle Chasse, La. & 48 & 17 & 4.1 & 32 & 1.7 \\
\hline
\end{tabular}

Table 77.- Concentration data for dissolved major elements in ultrafilter permeate from water samples collected from the Mississippi River and some of its tributaries during the March-April 1989 cruise

[ All results are in milligrams per liter ]

\begin{tabular}{|c|c|c|c|c|c|c|}
\hline $\begin{array}{l}\text { Date } \\
1989\end{array}$ & Sampling site & Calcium & Magnesium & Potassium & Sodium & $\begin{array}{c}\text { Silicon } \\
\text { (as } \mathrm{SiO}_{2} \text { ) }\end{array}$ \\
\hline 3-09 & Illinois River at Hardin, Ill. & 79 & 31 & 4 & 72 & 5 \\
\hline $3-10$ & Mississippi River near Winfield, Mo. & 50 & 21 & 3.3 & 18 & 4.6 \\
\hline $3-12$ & Missouri River at Hermann, Mo. & 42 & 16 & 3.4 & 30 & 8.7 \\
\hline $3-13$ & Mississippi River at St. Louis, Mo. & 52 & 20 & 3.8 & 36 & 5.4 \\
\hline $3-15$ & Mississippi River at Thebes, Ill. & 48 & 19 & 3.6 & 31 & 5.7 \\
\hline $3-16$ & Ohio River at Olmsted, Ill. & 26 & 6.1 & 1.6 & 7.2 & 5.3 \\
\hline 3-17 & Mississippi River below Hickman, Ky. & 31 & 8.7 & 1.9 & 12 & 5.3 \\
\hline $3-19$ & Mississippi River below Fulton, Tenn. & 30 & 8.3 & 2.1 & 11 & 5 \\
\hline $3-21$ & Mississippi River at Helena, Ark. & 30 & 8.4 & 2.1 & 11 & 5.2 \\
\hline $3-22$ & White River at Mile 11.5, Ark. & 18 & 6.7 & 1.4 & 1.8 & 4.4 \\
\hline $3-23$ & Arkansas River at Pendleton, Ark. & 24 & 6.3 & 2 & 31 & 3.8 \\
\hline 3-24 & Mississippi River above Arkansas City, Ark. & 32 & 9.3 & 2.2 & 14 & 5.5 \\
\hline $3-26$ & Yazoo River below Steele Bayou, Miss. & 7 & 2.2 & 2.3 & 3.2 & 5.2 \\
\hline $3-27$ & Mississippi River below Vicksburg, Miss. & 32 & 8.9 & 2.2 & 14 & 5.5 \\
\hline $3-29$ & $\begin{array}{l}\text { Old River Outflow Channel near Knox } \\
\text { Landing, La. }\end{array}$ & 32 & 8.8 & 2.2 & 13 & 5.5 \\
\hline $3-30$ & Mississippi River near St. Francisville, La. & 31 & 8.8 & 2.3 & 14 & 5.4 \\
\hline $4-01$ & Mississippi River below Belle Chasse, La. & 31 & 8.6 & 2.3 & 15 & 5.5 \\
\hline
\end{tabular}


Table 78. - Concentration data for dissolved major elements in ultrafilter permeate from water samples collected from the Mississippi River and some of its tributaries during the June 1989 cruise

[ All results are in milligrams per liter ]

\begin{tabular}{|c|c|c|c|c|c|c|}
\hline $\begin{array}{l}\text { Date } \\
1989\end{array}$ & Sampling site & Calcium & Magnesium & Potasslum & Sodium & $\begin{array}{c}\text { Silicon } \\
\left(\mathrm{as} \mathrm{SiO}_{2}\right)\end{array}$ \\
\hline $6-04$ & Illinois River at Hardin, IIl. & 70 & 33 & 4.3 & 41 & 3.3 \\
\hline $6-05$ & Mississippi River near Winfield, Mo. & 37 & 19 & 3.1 & 12 & 1.2 \\
\hline 6-07 & Missouri River at Hermann, Mo. & 53 & 20 & 4.9 & 52 & 7.7 \\
\hline 6-08 & Mississippi River at St. Louis, Mo. & 48 & 20 & 4 & 33 & 4.6 \\
\hline $6-10$ & Mississippi River at Thebes, Ill. & 48 & 20 & 3.9 & 32 & 4.2 \\
\hline $6-11$ & Ohio River at Olmsted, Ill. & 38 & 12 & 2.4 & 9.2 & 5.1 \\
\hline $6-12$ & Mississippi River below Hickman, Ky. & 41 & 15 & 3.2 & 18 & 4.7 \\
\hline 6-14 & Mississippi River at Fulton, Tenn. & 41 & 14 & 3 & 17 & 4.9 \\
\hline $6-17$ & Mississippi River at Helena, Ark. & 43 & 15 & 3 & 16 & 6.4 \\
\hline $6-18$ & White River at Mile 11.5, Ark. & 28 & 14 & 1.8 & 3.3 & 10 \\
\hline $6-20$ & Mississippi River above Arkansas City, Ark. & 34 & 12 & 2.6 & 22 & 5.7 \\
\hline $6-22$ & Yazoo River below Steele Bayou, Miss. & 5.5 & 1.8 & 2.6 & 2.6 & 7 \\
\hline $6-23$ & Mississippi River below Vicksburg, Miss. & 33 & 9.7 & 2.6 & 20 & 5.6 \\
\hline $6-25$ & $\begin{array}{l}\text { Old River Outflow Channel near Knox } \\
\text { Landing, La. }\end{array}$ & 32 & 9.4 & 2.6 & 19 & 5.6 \\
\hline $6-26$ & Mississippi River near St. Francisville, La. & 30 & 8.8 & 2.5 & 18 & 5.3 \\
\hline $6-28$ & Mississippi River below Belle Chasse, La. & 32 & 9.4 & 2.5 & 22 & 5.3 \\
\hline
\end{tabular}

Table 79.- Concentration data for dissolved major elements in ultrafilter permeate from water samples collected from the Mississippi River and some of its tributaries during the FebruaryMarch 1990 cruise

[ All results are in milligrams per liter ]

\begin{tabular}{|c|c|c|c|c|c|c|}
\hline $\begin{array}{l}\text { Date } \\
1990\end{array}$ & Sampling site & Calcium & Magneslum & Potasslum & Sodium & $\begin{array}{c}\text { Sllicon } \\
\text { (as } \mathrm{SiO}_{2} \text { ) }\end{array}$ \\
\hline $2-23$ & Cumberland River near Smithland, Ky. & 31 & 4.3 & 1.4 & 3 & 5.2 \\
\hline $2-24$ & Tennessee River near Calvert City, Ky. & 14 & 2.4 & 1.4 & 3.1 & 4.6 \\
\hline $2-25$ & Mississippi River near Cache, Ill. & 44 & 20 & 4.4 & 25 & 6.9 \\
\hline $2-28$ & Wabash River near New Haven, 111. & 48 & 17 & 3.2 & 8.4 & 7.8 \\
\hline 3-01 & Ohio River at Uniontown, Ky. & 31 & 8.4 & 2.3 & 8.7 & 6.3 \\
\hline 3-03 & Ohio River at Olmsted, IIl. & 27 & 7.2 & 2.1 & 6.5 & 5.9 \\
\hline 3-04 & Mississippi River below Hickman, Ky. & 32 & 11 & 2.6 & 10 & 6.3 \\
\hline $3-05$ & Mississippi River below Fulton, Tenn. & 33 & 11 & 2.6 & 11 & 6.9 \\
\hline 3-07 & Mississippi River at Helena, Ark. & 34 & 12 & 2.3 & 11 & 7.1 \\
\hline 3-08 & Mississippi River above Arkansas City, Ark. & 25 & 8.1 & 2.4 & 9.8 & 5.6 \\
\hline $3-10$ & Mississippi River below Vicksburg, Miss. & 27 & 8.5 & 2.4 & 11 & 5.7 \\
\hline $3-12$ & Mississippi River near St. Francisville, La. & 26 & 8 & 2.4 & 10 & 5.6 \\
\hline $3-14$ & Mississippi River below Belle Chasse, La. & 26 & 8.2 & 2.5 & 12 & 5.9 \\
\hline
\end{tabular}


Table 80.- Concentration data for dissolved major eiements in ultrafilter permeate from water samples collected from the Mississippi River and some of its tributaries during the May-June 1990 cruise

[ All results are in milligrams per liter ]

\begin{tabular}{|c|c|c|c|c|c|c|}
\hline $\begin{array}{l}\text { Date } \\
1990\end{array}$ & Sampling site & Calcium & Maanesium & Potassium & Sodium & sillcon \\
\hline $6-07$ & Illinois River at Valley City, Ill. & 76 & 33 & 2.9 & 26 & 6.3 \\
\hline $6-11$ & Mississippi River below Grafton, Ill. & 51 & 19 & 3.4 & 12 & 6.5 \\
\hline $6-13$ & Mississippi River at Thebes, Ill. & 38 & 12 & 3.7 & 12 & 7.1 \\
\hline $6-14$ & Ohio River at Olmsted, Ill. & 33 & 8.1 & 2.6 & 7 & 5.4 \\
\hline $6-18$ & Mississippi River below Memphis, Tenn. & 36 & 11 & 3.1 & 11 & 6.4 \\
\hline $6-20$ & Mississippi River below Arkansas City, Ark. & 36 & 11 & 3.1 & 11 & 6.7 \\
\hline $6-22$ & Yazoo River below Steele Bayou, Miss. & 10 & 3.3 & 3.1 & 4.7 & 6.2 \\
\hline $6-23$ & Mississippi River below Vicksburg, Miss. & 35 & 9.9 & 3.2 & 10 & 6.7 \\
\hline $6-25$ & Mississippi River near St. Francisville, La. & 36 & 10 & 3.2 & 11 & 6.8 \\
\hline $6-27$ & Mississippi River below Belle Chasse, La. & 35 & 10 & 3.2 & 13 & 6.9 \\
\hline
\end{tabular}

